\title{
THE DESIGN OF BLOCKED FRACTIONAL FACTORIAL SPLIT-PLOT EXPERIMENTS
}

\author{
BY \\ ROBERT G. McLEOD
}

A Thesis Submitted to the Faculty of Graduate Studies

in Partial Fulfillment of the Requirements

for the Degree of

DOCTOR OF PHILOSOPHY

Department of Statistics

University of Manitoba

Winnipeg, Manitoba

(C) Robcrt G. McLeod, July 2003 


\section{Abstract}

In two-level fractional factorial (FF) screening experiments, if some factors are hardto-vary and others are easy-to-vary, subsequent randomization restrictions on the run-order of the experiment may lead to the use of fractional factorial split-plot (FFSP) designs. Blocked fractional factorial split-plot (BFFSP) designs arise when all runs cannot be performed under homogeneous conditions. In this thesis, both applied and theoretical properties of BFFSP designs are considered.

Three approaches to blocking FFSP designs are introduced. The first approach, "pure whole-plot blocking", requires that blocking variables be generated exclusively by whole-plot factors. The second approach, "separation", uses blocking generators that consist of sub-plot factors alone, or sub-plot factors in conjunction with wholeplot factors. The third approach, "mixed blocking", incorporates properties of both pure whole-plot blocking and separation.

For ranking FF designs for use in screening experiments, the quality of a design is often assessed by means of the minimum aberration (MA) criterion. We provide an extension of the MA criterion to the BFFSP design setting, and a catalog of MA BFFSP designs is constructed. Using properties of Hadamard matrices, in conjunction with our MA criterion, we also develop theoretical results to assist in the construction of large blocked fractional factorial split-plot designs from designs with considerably fewer factors.

When the objective of an experiment is robust design, the MA criterion is typically unable to select an appropriate BFFSP design. We develop an approach for ranking BFFSP designs in this situation, and a catalog is presented containing BFFSP designs ranked according to our selection criteria.

We also consider the consequences of "elevating" a sub-plot factor to the wholeplot level. When the attributes of aberration, the number of clear factor effects and the precision of effect estimates are considered, it is shown that elevation may be a good design strategy.

The research in this thesis was motivated by an industrial BFFSP experiment involving the chrome-plating of aircraft engine component parts. We discuss this motivating case study in detail. 


\section{Acknowledgments}

Firstly, I must graciously thank Dr. John Brewster for his guidance as an advisor. I am very sure that few graduate students, if any, have received the amount of support from one advisor as I have from him. I wish to thank him for including me in an industrial consulting project, in 1998, which eventually became the motivation for my doctoral research. Subsequent to this, Dr. Brewster willingly included me in many other projects with the Institute of Industrial Mathematical Sciences, thus allowing me to gain valuable statistical research experience. I wish also to express my thanks to Dr. Brewster for the incredible level of financial support I received through him.

I would be remiss not to thank my committee members for their time spent in reading my thesis. All suggestions and proposed revisions have been greatly appreciated. Therefore, many thanks to professors Ken Mount, Dean Slonowsky, Rob Craigen and Doug Montgomery.

Many friends have made the long road to my doctorate more enjoyable and spiritually rewarding-Tim, Nolan, Brad, Bart, Shane, Phil, Kelvin, Bryce, Brent, Adam, Jason and Bill have all been great friends to me. Also, in the past year I have been blessed with a new extended family. Mom and dad Sass as well as numerous other brothers and sisters-in-laws and nephews and nieces have been a constant source of encouragement to me.

I would like to especially thank my father for instilling in me the love for math and more importantly a love for the Lord. Also, my brother, Jaret (Trina and Anna!), has been an encouragement to me in a way that only a brother can. Our times fishing and hunting have been some of my most memorable experiences.

They say that behind every successful man is a strong woman; over the years that woman has been my mother. Mom, thanks for all the support. I'm sure you have spent just as many hours praying for me as I have spent completing my Ph.D.

Finally, to the love of my life, Shanny; thanks for saying "yes". Keep on smiling and keep on loving the Lord. I look forward to spending the rest of our lives together. Thanks for being my best friend ...

For what profit is it to a man if he gains the whole world, and loses his own soul? Or what will a man give in exchange for his soul? Matthew 16:26 


\section{Contents}

1 Overview and Summary 1

1.1 Motivating Case Study . . . . . . . . . . . . . . . . . 2

1.2 Outline of Thesis . . . . . . . . . . . . . . 4

2 Design Preliminaries 7

2.1 Two-level Full Factorial Designs . . . . . . . . . . . . . . . . 8

2.2 Two-level Fractional Factorial Designs . . . . . . . . . . . . . . . . 10

2.3 Blocked Two-level Full Factorial Designs . . . . . . . . . . . . . . . . 14

2.4 Blocked Two-level Fractional Factorial Designs . . . . . . . . . . . . . 16

2.5 Split-plot Designs . . . . . . . . . . . . . . . . . . . 18

2.6 Two-level Fractional Factorial Split-plot Designs . . . . . . . . . . . . 21

2.6.1 Full Factorial Split-plot Designs . . . . . . . . . . . . . . 21

2.6.2 Fractional Factorial Split-plot Designs . . . . . . . . . . . . 21

2.6.3 Minimum Aberration and Rank-ordering FFSP Designs . . . . 22

3 BFFSP Designs and MA 25

3.1 Three Approaches to Constructing Blocking Variables . . . . . . . . . 25

3.1.1 Pure Whole-Plot Blocking . . . . . . . . . . . . . 26

3.1 .2 Separation . . . . . . . . . . . . . . . . 28

3.1.3 Mixed Blocking . . . . . . . . . . . . . . . 31

3.2 MA for BFFSP Designs . . . . . . . . . . . . . . . . . 31

3.2.1 A Limitation on the Word Length Definition . . . . . . . . . . 34

3.2.2 Ineligible and Impractical Designs . . . . . . . . . . . . . . . . 34

4 Variance Forms for Split-plot Effects 36

4.1 Derivation of Effect Variances . . . . . . . . . . . . . . . . 37 
4.1.1 Variance Forms for FFSP Designs . . . . . . . . . . . 37

4.1.2 Variance Forms for BFFSP Designs with Pure WP Blocking . 38

4.1.3 Variance Forms for BFFSP Designs with Blocking via Separation 42

4.1.4 Variance Forms for BFFSP Designs with Mixed Blocking . . . 44

4.2 Summary of Results . . . . . . . . . . . . . . . . . 47

5 MA BFFSP Design Search $\quad 49$

5.1 An Introduction to Search Algorithms and Related Issues . . . . . . . 49

5.1.1 Search-Table Approach for FFSP Designs . . . . . . . . 50

5.1.2 Design Isomorphism and Practical Considerations . . . . . . . 51

5.2 The Search-Table Approach for BFFSP Designs . . . . . . . . . . . 52

5.2.1 The Search-Table for Pure WP Blocking . . . . . . . . . . 52

5.2 .2 The Search-Table for Separation . . . . . . . . . . . . . 53

5.2 .3 The Search-Table for Mixed Blocking . . . . . . . . . 54

5.3 Additional Optimality Criteria . . . . . . . . . . . . . . . 57

5.4 A Catalog of MA BFFSP Designs . . . . . . . . . . . . 57

5.4.1 Overview of the MA Design Search Algorithm . . . . . . . 58

5.4.2 A Note on the Incompleteness of the MA BFFSP Design Catalog 59

6 Analysis of Variance for BFFSP Designs 61

6.1 ANOVA Models for $2^{k}$ Designs . . . . . . . . . . . 61

6.2 ANOVA Basics for Other Two-level Designs . . . . . . . . . . . 65

6.3 ANOVA Models for Two-level FFSP Designs . . . . . . . . . . . . 66

6.3.1 Two Approaches . . . . . . . . . . . . . . 6 66

6.4 ANOVA for Unreplicated BFFSP Designs . . . . . . . . . . . 68

6.4.1 Some Comments Regarding Unreplicated Designs . . . . . . 68

6.4.2 The ANOVA Model for Pure WP Blocking . . . . . . . . . 69

6.4.3 The ANOVA Model for Blocking via Separation . . . . . . . 71

6.4.4 The ANOVA Model for Mixed Blocking . . . . . . . . . . 74

6.5 ANOVA for Replicated BFFSP Designs . . . . . . . . . . . 77

6.5.1 The ANOVA Model for Pure WP Blocking: With Replication 77

6.5.2 The ANOVA Model for Blocking via Separation: With Replication . . . . . . . . . . . . . . 80

6.5.3 The ANOVA Model for Mixed Blocking: With Replication . . 82 
7 Results Analogous to Those for FFSP Designs $\quad 84$

7.1 Matrix Representation of BFFSP Designs: Notational Development . 84

7.2 Development of a General Matrix Representation for BFFSP Designs 87

7.3 Main Results . . . . . . . . . . . . . . . . . . . . . . . 89

7.3.1 Adding Both WP and SP Factors to an Existing BFFSP Design 90

7.3.2 Adding Only SP Factors to an Existing BFFSP Design . . . . 96

7.3.3 Comments Regarding Theorems 7.4 and $7.8 \ldots \ldots . \ldots 103$

8 Optimal BFFSP Experiments for RPD 104

8.1 Effect Orderings for FF and FFSP RPDs . . . . . . . . . . 106

8.1.1 Effect Ordering for FF RPDs _ . . . . . . . . . . . 107

8.1.2 Effect Ordering for FFSP RPDs . . . . . . . . . . . . . . 108

8.2 Effect Ordering for BFFSP RPDs . . . . . . . . . . . . 110

8.2.1 Implementation of the Effect Ordering for BFFSP RPDs . . . 111

8.2.2 A One-Number Optimality Criterion for BFFSP RPDs . . . . 112

8.2.3 Overview of the RPD Search Algorithm . . . . . . . . 113

8.3 Using the Optimal BFFSP RPD Catalog . . . . . . . . . . . . . 115

8.4 Limited Theoretical Results for RPDs . . . . . . . . . . . . . . . . 117

9 The Chrome-Plating Experiment Revisited 121

9.1 Three Competing Designs . . . . . . . . . . . . . . . . . . . . 121

9.1.1 Comparison of Designs . . . . . . . . . . . . . . . . 122

9.1 .2 Practical Considerations . . . . . . . . . . . . . . 124

10 Elevation $\quad 126$

10.1 Elevation Vs. Separation: As Motivated by the Chrome-Plating Experiment . . . . . . . . . . . . . . . . . . . . 127

10.1.1 Some Comments and an Example . . . . . . . . . . . . 129

10.1.2 The Initial, Elevated and Separated BFFSP Design Catalog . 133

10.1.3 Additional Comments . . . . . . . . . . . . . . . . . . 135

10.2 Elevation: Another Approach _. . . . . . . . . . . . 136

10.2.1 The Initial and Elevated BFFSP Design Catalog . . . . . . . 139

10.2.2 Additional Comments . . . . . . . . . . . . . . 140

A Standard Run Order of Selected Designs 142 
B Catalog of MA BFFSP Designs

B.1 Catalog of MA 8-Run BFFSP Designs via Pure WP Blocking . . . 154

B.2 Catalog of MA 16-Run BFFSP Designs via Pure WP Blocking . . . . 156

B.3 Catalog of MA 32-Run BFFSP Designs via Pure WP Blocking . . . . 161

B.4 Catalog of MA 8-Run BFFSP Designs via Separation . . . . . . . 167

B.5 Catalog of MA 16-Run BFFSP Designs via Separation . . . . . . 169

B.6 Catalog of MA 32-Run BFFSP Designs via Separation . . . . . . 173

B.7 Catalog of MA 16-Run BFFSP Designs via Mixed Blocking . . . . . 180

B.8 Catalog of MA 32-Run BFFSP Designs via Mixed Blocking . . . . . 183

$\begin{array}{ll}\text { C Initial, Elevated and Separated Designs } & 187\end{array}$

D Initial and Elevated Designs $\quad 202$

E Catalog of Optimal BFFSP RPDs $\quad 212$

E.1 Catalog of Optimal 16-Run BFFSP RPDs via Pure WP Blocking: Control Factors as SP Factors . . . . . . . . . . . . . . . . 213

E.2 Catalog of Optimal 32-Run BFFSP RPDs via Pure WP Blocking: Control Factors as SP Factors . . . . . . . . . . . . . . 217

E.3 Catalog of Optimal 16-Run BFFSP RPDs via Pure WP Blocking: Control Factors as WP Factors . . . . . . . . . . . . . . . . 221

E.4 Catalog of Optimal 32-Run BFFSP RPDs via Pure WP Blocking: Control Factors as WP Factors . . . . . . . . . . . . . . 225

E.5 Catalog of Optimal 16-Run BFFSP RPDs via Separation: Control Factors as SP Factors . . . . . . . . . . . . . . . . 229

E.6 Catalog of Optimal 32-Run BFFSP RPDs via Separation: Control Factors as SP Factors . . . . . . . . . . . . . . . . . . 233

E.7 Catalog of Optimal 16-Run BFFSP RPDs via Separation: Control Factors as WP Factors . . . . . . . . . . . . . . 236

E.8 Catalog of Optimal 32-Run BFFSP RPDs via Separation: Control Factors as WP Factors . . . . . . . . . . . . . . . . 239

E.9 Catalog of Optimal 16-Run BFFSP RPDs via Mixed Blocking: Control Factors as SP Factors . . . . . . . . . . . . . . . . . . . 242 
E.10 Catalog of Optimal 32-Run BFFSP RPDs via Mixed Blocking: Control Factors as SP Factors . . . . . . . . . . . . . . . . . . 245

E.11 Catalog of Optimal 16-Run BFFSP RPDs via Mixed Blocking: Control Factors as WP Factors . . . . . . . . . . . . . . . . . . . . 248

E.12 Catalog of Optimal 32-Run BFFSP RPDs via Mixed Blocking: Control Factors as WP Factors . . . . . . . . . . . . . . . . 250 


\section{List of Figures}

2.1 The Design Matrix for the $2^{4}$ Design. . . . . . . . . . . . . 13

2.2 A Simple Split-plot Experiment. . . . . . . . . . . . . . . . 19

2.3 A $2^{(3+3)-(1+1)}$ FFSP Design. . . . . . . . . . . . . . 23

3.1 Geometric Representation of the $2^{(3+3)-(0+1) \pm(1+0)}$ BFFSP Design. . . 27

3.2 Geometric Representation of the $2^{(3+3)-(0+1) \pm(0+1)}$ BFFSP Design. . . 30

3.3 Geometric Representation of the $2^{(3+3)-(0+1) \pm(1+1)}$ BFFSP Design. . . 32

6.1 The Design Matrix for the Replicated $2^{3}$ Design. . . . . . . . . . . 62

9.1 The Three Competing Scenarios, Illustrating Elevation and Separation 122

10.1 Elevation Vs. Separation: As Motivated by the Chrome-Plating Ex-

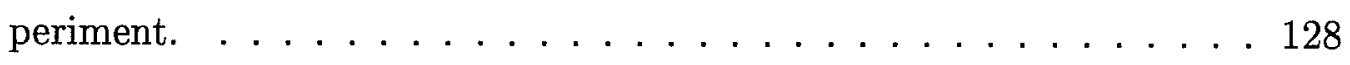

10.2 Elevation: Another Approach . . . . . . . . . . . . 137 


\section{List of Tables}

2.1 Running an Experiment in Four Blocks . . . . . . . . . . . 15

2.2 Stucture of the ANOVA Table for the Two-factor Split-plot Experiment in Example 2.5.1. . . . . . . . . . . . . . . . . 20

4.1 Variance Forms of WP and SP Effects in FFSP and BFFSP Designs. 48

5.1 The Search-Table for the $2^{(5+4)-(2+3)}$ Design in Example 5.1.1. . . . 51

5.2 The Search-Table for the $2^{(4+2)-(1+1) \pm(1+0)}$ Design in Example 5.2.1. . 53

5.3 The Search-Table for the $2^{(4+3)-(1+1) \pm(0+1)}$ Design in Example 5.2.2. . 55

5.4 The Search-Table for the $2^{(4+3)-(1+1) \pm(1+1)}$ Design in Example 5.2.3. . 56

6.1 ANOVA for the Replicated $2^{3}$ Design in Example 6.1.1. . . . . . . . 64

6.2 An ANOVA Model for the $2^{\left(n_{1}+n_{2}\right)-\left(k_{1}+k_{2}\right)}$ FFSP Design. . . . . . . 67

6.3 An Alternate ANOVA Model for the $2^{\left(n_{1}+n_{2}\right)-\left(k_{1}+k_{2}\right)}$ FFSP Design. . . 68

6.4 The ANOVA Model for the $2^{\left(n_{1}+n_{2}\right)-\left(k_{1}+k_{2}\right) \pm\left(b_{1}+0\right)}$ BFFSP Design. . . 70

6.5 Alias Structure of the $2^{(3+3)-(0+1) \pm(1+0)}$ BFFSP Design in Example 6.4.1. 72

6.6 The ANOVA Model for the $2^{\left(n_{1}+n_{2}\right)-\left(k_{1}+k_{2}\right) \pm\left(0+b_{2}\right)}$ BFFSP Design. . . 73

6.7 Alias Structure of the $2^{(3+3)-(0+1) \pm(0+1)}$ BFFSP Design in Example 6.4.2. 75

6.8 The ANOVA Model for the $2^{\left(n_{1}+n_{2}\right)-\left(k_{1}+k_{2}\right) \pm\left(b_{1}+b_{2}\right)}$ BFFSP Design. . . 76

6.9 Alias Structure of the $2^{(3+3)-(0+1) \pm(1+1)}$ BFFSP Design in Example 6.4.3. 78

6.10 ANOVA Model for a Replicated $2^{\left(n_{1}+n_{2}\right)-\left(k_{1}+k_{2}\right) \pm\left(b_{1}+0\right)}$ BFFSP Design. 79

6.11 ANOVA Model for a Replicated $2^{\left(n_{1}+n_{2}\right)-\left(k_{1}+k_{2}\right) \pm\left(0+b_{2}\right)}$ BFFSP Design. 81

6.12 ANOVA Model for a Replicated $2^{\left(n_{1}+n_{2}\right)-\left(k_{1}+k_{2}\right) \pm\left(b_{1}+b_{2}\right)}$ BFFSP Design. 83

7.1 Matrix Representation of the DCS for the $2^{(5+3)-(1+1) \pm(1+1)}$ Design . . 85

8.1 Effect Rankings for FFSP RPDs . . . . . . . . . . . . . . . 108

8.2 Compromise Ranking Scheme with Associated Word-lengths . . . . 109 
8.3 Effect and Effect Precision Rankings for BFFSP RPDs . . . . . . 110

9.1 Precision of Effect Estimates and Alias Structures for the Three Designs123

9.2 Variances of Estimated Effects for Designs $D, E$ and $S \ldots \ldots 124$

10.1 Precision of Effect Estimates and Alias Structures for the Initial, Elevated and Separated Designs in Example 10.1.1. . . . . . . . . 131

A.1 Standard Run Order of the $2^{(3+3)-(0+1)}$ FFSP Design. . . . . . . . 143

A.2 Standard Run Order of the $2^{(3+3)-(0+1) \pm(1+0)}$ BFFSP Design. . . . . 145

A.3 Standard Run Order of the $2^{(3+3)-(0+1) \pm(0+1)}$ BFFSP Design. . . . . 147

A.4 Standard Run Order of the $2^{(3+3)-(0+1) \pm(1+1)}$ BFFSP Design. . . . . 149

A.5 Standard Run Order of the Impractical $2^{(4+2)-(0+1) \pm(2+1)}$ BFFSP Design. 151

B.1 MA 8-Run BFFSP Designs via Pure WP Blocking. . . . . . . . . 155

B.2 MA 16-Run BFFSP Designs via Pure WP Blocking. . . . . . . . . 157

B.3 MA 32-Run BFFSP Designs via Pure WP Blocking. . . . . . . . . . 162

B.4 MA 8-Run BFFSP Designs via Separation. . . . . . . . . . . 168

B.5 MA 16-Run BFFSP Designs via Separation. . . . . . . . . . 170

B.6 MA 32-Run BFFSP Designs via Separation. . . . . . . . . . . 174

B.7 MA 16-Run BFFSP Designs via Mixed Blocking. . . . . . . . . 181

B.8 MA 32-Run BFFSP Designs via Mixed Blocking. . . . . . . . . . . 184

C.1 32-Run Triples: Initial Design is an MA FFSP Design . . . . . . . 188

C.2 32-Run Triples: Initial Design is an MA BFFSP Design with Pure WP Blocking . . . . . . . . . . . . . . . . . . 193

C.3 32-Run Triples: Initial Design is an MA BFFSP Design with Separation 197

C.4 32-Run Triples: Initial Design is an MA BFFSP Design with Mixed Blocking . . . . . . . . . . . . . . . . . 201

D.1 32-Run Pairs: Elevation of an MA Initial Design with Pure WP Blocking to an MA Design with Pure WP Blocking . . . . . . . . . 203

D.2 32-Run Pairs: Elevation of a Separated MA Initial Design to a Separated MA Design . . . . . . . . . . . . . . . . . 206

D.3 32-Run Pairs: Elevation of a Separated MA Initial Design to an MA Design with Pure WP Blocking . . . . . . . . . . . 208 
D.4 32-Run Pairs: Elevation of an MA Initial Design with Pure WP Blocking to a Separated MA Design . . . . . . . . . . . . . . . . 210

E.1 Optimal 16-Run BFFSP RPDs via Pure WP Blocking: Control Factors as SP Factors . . . . . . . . . . . . . . . . . . . . 214

E.2 Optimal 32-Run BFFSP RPDs via Pure WP Blocking: Control Factors as SP Factors . . . . . . . . . . . . . . . . . . . . 218

E.3 Optimal 16-Run BFFSP RPDs via Pure WP Blocking: Control Factors as WP Factors . . . . . . . . . . . . . . . . . . 222

E.4 Optimal 32-Run BFFSP RPDs via Pure WP Blocking: Control Factors as WP Factors . . . . . . . . . . . . . . . . . 226

E.5 Optimal 16-Run BFFSP RPDs via Separation: Control Factors as SP Factors . . . . . . . . . . . . . . . . . . . . 230

E.6 Optimal 32-Run BFFSP RPDs via Separation: Control Factors as SP Factors . . . . . . . . . . . . . . . . . . . . . 234

E.7 Optimal 16-Run BFFSP RPDs via Separation: Control Factors as WP Factors . . . . . . . . . . . . . . . . . . . . 237

E.8 Optimal 32-Run BFFSP RPDs via Separation: Control Factors as WP Factors . . . . . . . . . . . . . . . . . . . . . . 240

E.9 Optimal 16-Run BFFSP RPDs via Mixed Blocking: Control Factors as SP Factors . . . . . . . . . . . . . . . . . . . 243

E.10 Optimal 32-Run BFFSP RPDs via Mixed Blocking: Control Factors as SP Factors . . . . . . . . . . . . . . . . . . 246

E.11 Optimal 16-Run BFFSP RPDs via Mixed Blocking: Control Factors as WP Factors . . . . . . . . . . . . . . . . . . . . 249

E.12 Optimal 32-Run BFFSP RPDs via Mixed Blocking: Control Factors

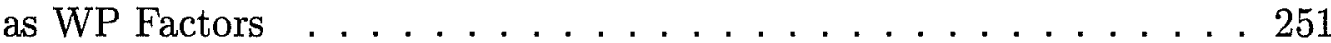




\section{Chapter 1}

\section{Overview and Summary}

Performing screening experiments by means of two-level fractional factorial (FF) designs has been well documented over the years (Cox, 1958; Box, Hunter and Hunter, 1978; Montgomery, 2001). However, if it is expensive, time consuming or in some way labor intensive to change the levels of some of the factors, it may be impractical or even impossible to perform the experimental runs of the $\mathrm{FF}$ in a completely random order. Under these circumstances, the randomization restrictions that are imposed in the design of the experiment may result in a split-plot structure. In such cases fractional factorial split-plot (FFSP) designs arise (Bingham and Sitter, 1999a; 2001). FFSP designs also arise when different factors are applied to different sizes of experimental units. Split-plot designs were originally motivated by agricultural experiments with the whole-plots (WPs) usually being large areas of land and the subplots (SPs) being smaller portions of land within the large areas (Yates, 1935).

Two FFs underlie the structure of a FFSP design - the $n_{1}$ WP or hard-to-vary factors arranged as a $2^{n_{1}-k_{1}} \mathrm{FF}$ and the $n_{2} \mathrm{SP}$ or easy-to-vary factors arranged as a $2^{n_{2}-k_{2}} \mathrm{FF}$. However, if one views the WP and SP designs separately, there are limited design options (Bingham and Sitter, 1999a). Rather, it is better if WP factors are included in the SP factor generators (Kempthorne, 1952, p. 318). This approach often allows one to increase the resolution $(R)$ of a FFSP design. A two-level FFSP design is typically denoted by $2^{\left(n_{1}+n_{2}\right)-\left(k_{1}+k_{2}\right)}$; this notation parallels that of Huang, Chen and Voelkel (1998) and Bingham and Sitter (1999a, 2001) and implies that there are $n=n_{1}+n_{2}$ treatment factors, $k=k_{1}+k_{2}$ "added" factors and $n_{1}+n_{2}-k_{1}-k_{2}$ "basic" factors from which the added factors are generated. Chapter 2 provides an 
extensive summary of these introductory design issues.

If it is thought that experimental conditions will not remain homogeneous for all $2^{\left(n_{1}+n_{2}\right)-\left(k_{1}+k_{2}\right)}$ runs of the FFSP design, blocking may represent a practical design option. For example, if a single batch of raw material is not large enough to permit all $2^{\left(n_{1}+n_{2}\right)-\left(k_{1}+k_{2}\right)}$ treatment combinations to be run, then blocking may be used to account for variability between batches. Industrial experiments may also be blocked using specific time periods (for example, blocked by week). This enables researchers to account for variability that occurs in a process over time, including variability due to changing environmental conditions or personnel.

The research in this thesis arose from a blocked fractional factorial split-plot (BFFSP) experiment involving the chrome-plating of aircraft engine component parts. The following section provides an introduction to this experiment. We conclude the chapter with an outline of the research completed in this thesis.

\subsection{Motivating Case Study}

An aerospace company was experiencing problems with one of its chrome-plating processes in that, when a particular complex-shaped part was being plated, excessive pitting and cracking were observed. In addition, poor adhesion and uneven deposition of chrome across the part were detected. With the goal being the identification of key factors affecting the quality of the chrome-plating process, it was decided that a screening experiment would be conducted.

In collaboration with the company's process engineers, six factors were identified for consideration in the experiment: $A=$ chrome concentration, $B=$ chrome to sulfate ratio, $C=$ bath temperature, $p=$ etching current density, $q=$ plating current density, and $r=$ part geometry. The responses included the number of pits and number of cracks, in addition to hardness and thickness readings at various locations on the part.

Factors $A, B$ and $C$ were hard-to-vary in that they represented characteristics of the bath in which the chrome-plating was being carried out. It was difficult to change the composition or temperature of the bath quickly, as changes could be made at most once per day. Moreover, there was only one tank, or bath, available for the experiment. 
Factors $p, q$ and $r$ were easy-to-vary in that they could be changed multiple times per day at the rectifier level. A rectifier is a bar through which current passes, and is the cathode for the plating process. There were a number of rectifiers in the tank, and different levels of $p, q$ and $r$ could be used on each rectifier.

The fact that some factors were harder-to-vary than others led to restrictions on randomization. This in turn led to the use of a split-plot design, with $A, B$ and $C$ being the WP factors and $p, q$ and $r$ being the SP factors. Because this was a screening experiment a two-level design was used, with high and low levels being selected for each factor. A full unreplicated factorial experiment would require eight days, there would be eight rectifiers in the tank, and, on each day, one part would be plated on each rectifier. In total, $2^{6}=64$ parts would be plated.

However, there were only four rectifiers in the tank, and it was desirable to use only two of these - with the other two being used for a separate experiment. Therefore, at first glance, we were faced with a $2^{3-2}$ design at the SP level. On the positive side, sufficient resources were available to run the experiment for 16 days, and to plate two parts per day. These 16 days consisted of four four-day weeks and it was desirable to block the experiment by week.

In constructing the design a decision was made to raise (elevate) one of the SP factors, $p$, to the WP level (and to denote it by $P$ ). Thus, etching current was only varied at the day level. On the one hand, by raising etching current to the WP level it was recognized that less information would be available about this factor, because it would now be tested against the WP error rather than the SP error. On the other hand, a certain level of simplicity would be achieved, in that we could now think of the experiment at the WP level as a full, unreplicated, $2^{4}$ design, run in four blocks. As well, we would have less fractionation at the SP level, as we could now think of the experiment at the SP level as a one-half fraction of a $2^{2}$ design (although not crossed with the WP design).

The design that was run was an unreplicated $2^{(4+2)-(0+1)}$ in $2^{2}$ blocks. One factor generator, $r=A B C P q$, was used to generate the SP added factor $r$. Two pure WP blocking variables, $\beta_{1}=A B C$ and $\beta_{2}=A B P$, were used to generate the four blocks and, as a consequence, one two-factor interaction (2fi), $C P$, was also confounded with blocks.

Questions concerning the optimality of the design led to the current research. We 
were particularly interested in whether the chosen (factor and block) generators were "optimal", and whether it was a good idea to raise $p$ to the WP level (or whether better designs could be constructed by leaving $p$ at the SP level). We will revisit these issues, in the context of the chrome-plating experiment, in Chapters 9 and 10. Before doing this we will develop a more general theoretical framework in Chapters 2 $-8$.

\subsection{Outline of Thesis}

Chapter 2 provides an overview of FF, blocked fractional factorial (BFF) and FFSP designs. This overview will provide the reader with a reasonable foundation for studying more advanced designs in subsequent chapters.

Blocking FFSP designs is complicated by the presence of the two FFs underlying the split-plot structure. We will demonstrate that there is more than one way to block a FFSP design. In Chapter 3 we will show that one may induce blocking at the WP level using three distinct, yet related, approaches. The first approach, "pure WP blocking", requires that blocking variables be generated exclusively by WP factors. The second approach, which we call "separation", uses blocking generators that consist of SP factors alone, or SP factors in conjunction with WP factors. The third approach, "mixed blocking", incorporates properties of both pure WP blocking and separation.

In this context, the concept of separation appears to be new. The key point is that the blocking generators, or "separators", are used to induce blocking at the WP level by grouping all of the runs from the same block together. However, the motivation for separation goes beyond the obvious intent of grouping treatment combinations. In essence, the process of separation achieves "pseudo-replication" at the WP level at the expense of further fractionation at the SP level (Brewster and McLeod, 2000).

In Chapter 3 we also provide a straightforward extension of the minimum aberration (MA) criterion to the blocked fractional factorial split-plot (BFFSP) design setting. Our approach is based upon that of Sitter, Chen and Feder (1997) and enables a practitioner to select "good" BFFSP designs for the estimation of (WP and SP) main effects and two-factor interactions (2fi's).

When testing for the significance of an estimated split-plot effect one must test 
the given effect against the appropriate variance. If an incorrect variance is used, erroneous inferences regarding the statistical significance of that effect may occur. In Chapter 4, the variance forms for split-plot effects arising from the three blocking approaches are constructed and compared. These variance forms imply general rules for correctly assessing the significance of BFFSP design contrasts. These rules are an extension of those initially developed for the FFSP setting (Bisgaard, 2000; Bingham and Sitter, 2001).

Chapter 5 details methods by which one may algorithmically "search" for and obtain two-level BFFSP designs ranked according to the MA criterion. Our search algorithm is patterned after the search-table approach of Franklin and Bailey (1977) and the combined approach of Bingham and Sitter (1999a). From this search procedure we will see that for a given number of treatment factors, blocking variables and degree of fractionation there may be many BFFSP designs possessing MA. To further differentiate between the "goodness" of these MA designs additional optimality criteria (such as the number of clear SP main effects and 2fi's) will be presented for ranking purposes. These additional optimality criteria may be used to distinguish between MA BFFSP designs possessing the same word length pattern (WLP). A catalog is then constructed containing 8, 16 and 32-run BFFSP designs ranked according to the MA criterion and the aforementioned additional optimality criteria.

In experimental design it is well known that the analysis of variance (ANOVA) approach provides the experimenter with a versatile statistical tool for studying the relationship between a response variable and one or more (treatment) factors (Montgomery, 2001 and references therein). The main task of an ANOVA is to quantify and evaluate the importance of possible sources of variation in an underlying linear model. This is accomplished by forming a partition of the total sum of squares and degrees of freedom associated with the response variable, $y$, into its component parts. With this in mind, Chapter 6 provides an indepth look into the construction of ANOVA models for the three blocking approaches. Considerable time is then spent detailing appropriate methods for the testing of split-plot effects using the ANOVA models.

While FF and FFSP designs have been widely used for some time, the theoretical properties of "optimal" (that is, MA) FF and FFSP designs were, for the most part, undeveloped until recently. Chen and Wu (1991) and Bingham and Sitter (1999b) 
introduced novel matrix representations for the defining contrast subgroups (DCSs) of FF and FFSP designs, respectively. In Chapter 7 we take a theoretical look at BFFSP designs by developing a matrix representation for their DCSs that account for the presence of blocking variables. These results represent an extension of those for FF and FFSP designs. While adhering to the MA criterion, we develop theorems that allow BFFSP designs with many factors to be constructed from BFFSP designs with significantly fewer factors. In addition, theorems are presented which place lower bounds on a BFFSP design's maximum resolution. These results can be incorporated in design search algorithms to reduce search times.

In Chapter 8 we formulate an approach by which one may construct and subsequently rank BFFSP designs for the purpose of robust parameter design (RPD). In $\mathrm{RPD}$, an experimenter's objective is to reduce the variability in a product or process by a careful selection of factor level settings. We demonstrate that the MA criterion developed in Chapter 3 is not suitable for ranking BFFSP RPDs since it is unable to discriminate between the importance of effects having the same order. For example, our MA criterion cannot distinguish between the importance of control $\times$ control and control $\times$ noise 2fi's, whereas the latter are more important in RPD. Instead of using the MA criterion in our ranking procedure, we construct an effect ranking scheme that is a compromise between the notions of "likely significance" of effects and "effect interest". Our approach is an extension of that of Wu and Hamada (2000) and Bingham and Sitter (2003) for FF and FFSP RPDs, respectively. Using our ranking procedure 16 and 32-run optimal BFFSP RPDs are constructed. Examples are given illustrating the superiority of our effect ranking scheme over that of the MA criterion, when the objective is robust design.

Chapter 10 considers the effect of "elevating" a sub-plot factor to the whole-plot level in BFFSP designs. We demonstrate that elevation may lead to a design with higher estimation capacity. That is, an elevated design may be more appealing in terms of our proposed design optimality characteristics (aberration, number of clear effects, precision of effects). Underlying the elevation procedure is the assumption that the experimenter is not overly concerned with the loss of precision for the elevated factor as a consequence of running it at the WP level. Catalogs of 32-run BFFSP designs are constructed containing elevated designs that potentially provide the practitioner with superior estimation capacity. 


\section{Chapter 2}

\section{Design Preliminaries}

The importance of experimental design in agricultural (Yates, 1935; Kempthorne, 1952; Fisher, 1960), industrial (Box, Hunter and Hunter, 1978; Wu and Hamada, 2000; Montgomery, 2001) and other scientific applications (Lindquist, 1953; Kirk, 1968) has been well documented. The main objective of experimental design has been to develop methods by which one may simultaneously account for, or model, the effect of a set of factors (variables) comprising some process under investigation. The approach taken by today's proponents of experimental design methodology represents a radical departure from the "one-factor-at-a-time" investigative approach that was prevalent in many experiments during the early stages of the $20^{\text {th }}$ century. It is now well known that the major pitfall of one-factor-at-a-time experimentation is that it negates the opportunity to identify important interactions between experimental factors.

The objectives of experimental design usually fall under (at least) one of the following headings:

1. The determination of which factors exert an important influence on a particular process,

2. The determination of factor settings by which the process is optimized. (In experimental design, optimization may take on several forms, such as maximizing the yield or minimizing the variability of a process.)

The class of designs with $n$ factors each at two levels has been given much attention in the literature (see above references). These designs have been named " $2^{n}$ full 
factorial designs", since they consist of $\overbrace{2 * 2 * \ldots * 2}^{n}=2^{n}$ observations or treatment combinations. These factor combinations are varied together rather than one-at-atime. From this class of designs we obtain two-level fractional factorial (FF), blocked fractional factorial (BFF) and fractional factorial split-plot (FFSP) designs.

It is our intent in this chapter to provide an overview of FF, BFF and FFSP designs. This starting point will provide the required statistical background for dealing with more advanced designs in subsequent chapters.

\subsection{Two-level Full Factorial Designs}

A $2^{n}$ full factorial design is comprised of all possible $2^{n}$ combinations of the $n$ factors, where each factor has only two levels or settings. Therefore, a $2^{n}$ full factorial design consists of $2^{n}$ runs. When conducted, the $2^{n}$ full factorial experiment will provide information on all possible factorial effects, where a factorial effect is either a main effect or an interaction between two or more factors. A main effect refers to the change in response produced by a change in the levels of one of the primary experimental factors of interest (Montgomery, 2001). An interaction between two factors is said to occur if we find that the difference in response between the levels of one factor is not the same at all levels of the other factor.

The classic example by which many authors illustrate the basic attributes and analysis of a full factorial design is the $2^{3}$ design (Anderson and McLean, 1974; Bingham, 1998; Montgomery, 2001). We denote the three design factors by A, B and C. By using the usual " $+/$ - coding" for representing the high and low levels of the factors, we may list the $2^{3}=8$ runs as the rows of the following array: 


$$
\begin{aligned}
& A \quad B \quad C \\
& \left(\begin{array}{lll}
- & - & - \\
+ & - & - \\
- & + & - \\
+ & + & - \\
- & - & + \\
+ & - & + \\
- & + & + \\
+ & + & +
\end{array}\right) .
\end{aligned}
$$

This list of runs in terms of the coded factor levels is referred to as the design matrix. If the experimental design is displayed in terms of the actual factor levels the list of runs is sometimes referred to as the planning matrix.

One may display the entire set of columns corresponding to all main effects and interaction effects by use of the full factorial design matrix. For the $2^{3}$ design, the full factorial design matrix is given by

$$
\mathbf{X}=\left(\begin{array}{ccccccc}
A & B & C & A B & A C & B C & A B C \\
- & - & - & + & + & + & - \\
+ & - & - & - & - & + & + \\
- & + & - & - & + & - & + \\
+ & + & - & + & - & - & - \\
- & - & + & + & - & - & + \\
+ & - & + & - & + & - & - \\
- & + & + & - & - & + & - \\
+ & + & + & + & + & + & +
\end{array}\right),
$$

where $\mathrm{AB}, \mathrm{AC}, \mathrm{BC}$ and $\mathrm{ABC}$ represent all possible two-factor interactions (2fi's) and three-factor interactions (3fi's) of the $2^{3}$ design.

An estimated main effect or interaction, denoted by $\hat{c}$, is given by

$$
\hat{c}=\frac{\mathbf{x}_{\mathrm{c}}^{\prime} \mathbf{y}}{r 2^{n-1}},
$$

where $\mathbf{x}_{\mathbf{c}}$ is the column vector of $+/$ - signs in the full factorial design matrix corresponding to factor $c, \mathbf{y}$ is the vector of the response observations corresponding to 
the factor level settings in each row of $\mathbf{X}$, and $r$ is the number of replications of the $2^{n}$ design.

The usual model for a $2^{n}$ design may be summarized by

$$
y=f(\text { factor effects })+\epsilon,
$$

where $\epsilon$ is the random error term and $f(\cdot)$ is a linear function of the main effects and interactions.

\subsection{Two-level Fractional Factorial Designs}

One drawback of $2^{n}$ full factorial designs is their drain on resources. As $n$, the number of factors increases, it is readily apparent that the required number of experimental runs may become prohibitive. That is, expense, time and labour requirements demanded by large $2^{n}$ designs may be impossible to meet. By assuming that certain high-order interactions (for example, 3f's and 4f's) are negligible one may obtain information on all remaining main effects and interactions by running only a subset or fraction of the $2^{n}$ full factorial design. (The assumption that high-order interactions typically exert a negligible effect on a process has been empirically justified by many studies (Wu and Hamada, 2000) and is referred to as effect hierarchy.) These considerations have cemented the popularity of two-level FF designs; consequently, FF, rather than full factorial designs are the norm rather than the exception.

The analytic approach to FF designs (and that of factorial designs in general) is guided by several principles as listed below:

1. The sparsity of effects principle: When there are several variables the process is likely to be driven primarily by relatively few of the main effects and low-order interactions.

2. The projection property: FF designs can be projected into stronger designs in the subset of significant factors. If the number of significant factors is small, these "stronger designs" may possess replicated treatment combinations, thus allowing the formation of error estimates. (For a thorough description see Montgomery (2001), pp.246-251.)

3. Effect hierarchy: Main effects are more likely to be significant than 2fi's, 2fi's are more likely to be significant than 3fi's, and so on. 
4. Effect heredity: Interactions involving significant main effects are more likely to be significant than interactions involving main effects that are not significant.

In general, one defines a $2^{n-k}$ design to be a FF design with $n$ factors, each at two levels, and consisting of $2^{n-k}$ treatment combinations or runs. Since a $2^{n-k} \mathrm{FF}$ design is a $2^{-k}$-th fraction of the $2^{n}$ full factorial design, the main concern in constructing FF designs is the correct choice of factor level settings to be run. The correct factor level settings are determined by assigning $k$ of the factors to interaction columns of the $2^{n-k}$ full factorial design matrix. The first $n-k$ columns of the design matrix are considered to be independent and they determine the $2^{n-k}$ treatment combinations of the first $n-k$ basic factors. It is the columns representing the interactions of the first $n-k$ factors that enable the experimenter to determine the factor level settings for the remaining $k$ added (or dependent) factors.

Example 2.2.1 Consider a $2^{6-2}$ FF design. This design requires 16 runs whereas a $2^{6}$ full factorial design would require 64 . Hence, the FF design is a " $2^{-2}=\frac{1}{4}$ fraction" of the $2^{6}$ design. One would assign four basic factors, say $A, B, C$ and $D$, to the four (independent) columns of the $2^{4}$ design matrix given in Figure 2.1. One needs to assign the two remaining factors, say $E$ and $F$, to interactions involving $A, B, C$ and $D$. One possibility is $E=A B$ and $F=A C D$. This implies that the settings of factors $E$ and $F$ are completely determined by the columns corresponding to the interactions $A B$ and $A C D$, respectively.

From the properties of addition modulus 2 arithmetic it is seen that $I=A B E$ and $I=A C D F$ where $I$ is the identity column of 1 's (or + 's) and is obtained by multiplying both sides of $E=A B$ and $F=A C D$ by $E$ and $F$, respectively. We can combine the preceding relations by writing $I=A B E=A C D F$ where $E=A B$ and $F$ $=A C D$ are known as the generators of the $2^{6-2}$ design. By taking the product of the generators we obtain

$$
I=A B E=A C D F=B C D E F,
$$

which is called the (treatment) defining contrast subgroup or DCS of the design (Wu and Hamada, 2000), where ABE, ACDF and BCDEF form the set of words in the DCS. Note that many authors (Box, Hunter and Hunter, 1978; Hedayat, Sloane and Stufken, 1999; Montgomery, 2001) also refer to the DCS as the defining relation of a design. 
Since only a subset of the $2^{6}$ runs are to be performed we are in essence obtaining "less than complete information" for some of the main effects and interactions. For example, since the settings of $E$ and $F$ are determined by $A B$ and $A C D$, respectively, their factor effect estimates will be indistinguishable from these interactions. That is, $E$ is aliased with the $2 f A B$ and $F$ is aliased with the $3 f A C D$.

Aliasing is a direct consequence of running a $F F$ design and is always unavoidable. However, some forms of aliasing are considered to be worse than others. For example, consider the aliased effects, $E=A B$ and $F=A C D$. Here $E$ is aliased with a $2 f$ while $F$ is aliased with a $3 f$. In order to estimate the main effects $E$ and $F$ the interactions $A B$ and $A C D$ must be assumed negligible. Whether or not $A B$ and/or $A C D$ are truly negligible is the key issue. It was previously mentioned that empirical evidence suggests that higher order interactions are negligible. Therefore, it is "easier" to claim that the $3 f(A C D)$ is small and hence $F$ is estimable, than to claim that the $2 f(A B)$ is negligible.

One lesson to be drawn from Example 2.2.1 is that longer words in the DCS are "better". That is, one would wish to have as many long words in the DCS as possible since words consisting of only three factors (letters) cause main effects to be aliased with 2fi's, words consisting of four letters cause 2fi's to aliased with other 2fi's, five letter words cause 2 fi's to be aliased with 3fi's, and so on.

At this point it is useful to introduce the concepts of word length, word length pattern (WLP) and design resolution. The number of letters in a word is its word length and the vector

$$
W=\left(A_{1}, A_{2}, \ldots, A_{n}\right),
$$

is called the WLP of the design, where $A_{i}$ denotes the number of words of length $i$ in the design, D. The shortest word length in a design's DCS (excluding $I$ ) is called the resolution (Box and Hunter, 1961) of the design. The $2^{6-2} \mathrm{FF}$ design in Example 2.2.1 has WLP $W=(0,0,1,1,1,0)$ and thus is a resolution 3 design.

From the above discussion we understand that designs with larger resolutions are often preferred. However, knowledge of the resolution of a design is insufficient in assessing the estimation capacity of a design. For instance, designs with equal resolution do not necessarily have equal WLPs and consequently may not have the same alias structure. In order to differentiate between designs of equal resolution, 
Figure 2.1: The Design Matrix for the $2^{4}$ Design.

$$
\begin{gathered}
A \\
\left(\begin{array}{cccc}
- & - & - & - \\
+ & - & - & - \\
- & + & - & - \\
+ & + & - & - \\
- & - & + & - \\
+ & - & + & - \\
- & + & + & - \\
+ & + & + & - \\
- & - & - & + \\
+ & - & - & + \\
- & + & - & + \\
+ & + & - & + \\
- & - & + & + \\
+ & - & + & + \\
- & + & + & + \\
+ & + & + & +
\end{array}\right)
\end{gathered}
$$

Fries and Hunter (1980) developed a criterion entitled minimum aberration (MA). In the context of FF designs, MA has the following definition:

Definition 2.2.1 (Minimum Aberration) For two designs $D_{1}$ and $D_{2}$, let $r$ be the smallest value such that $A_{r}\left(D_{1}\right) \neq A_{r}\left(D_{2}\right)$. We say that $D_{1}$ has less aberration than $D_{2}$ if $A_{r}\left(D_{1}\right)<A_{r}\left(D_{2}\right)$. If there is no design with less aberration than $D_{1}$, then $D_{1}$ has the MA (treatment) DCS and therefore is a "MA design".

The MA criterion sequentially minimizes the number of words of length $A_{i}$ in the WLP of a FF design. Since a MA design will have as few short words as possible one will typically run a MA FF design rather than an equally sized $\mathrm{FF}$ design not possessing MA-exceptions to this "rule" will be considered in later chapters. 


\subsection{Blocked Two-level Full Factorial Designs}

Montgomery (2001) defines blocking in experimental design to be a technique used to increase the precision of an experiment. A block is the portion of the experimental material that is more homogeneous than the entire set of material. For example, an experiment in a manufacturing process may require two days to complete all the required runs. However, there could be differences between the days due to employeeto-employee (work shift) variability. If we are not specifically interested in this effect, we could think of "days" (work shifts) as blocks. Within a given day, we would expect experimental conditions to be more consistent (homogenous) given that the employees remain constant through the day. However, between days we may assume a larger degree of variability in the process due to an entirely different shift of employees.

In general, blocking serves to reduce the variability transmitted from sources other than the factors of interest in the underlying process as long as the within-block variation is smaller than the between-block variation. This variability reduction will increase the precision by which the experimenter may make subsequent statistical inferences regarding the treatment factors.

Sitter et al. (1997) and Loeppky and Sitter (2002) summarize the approach to blocking $2^{n}$ designs. First, consider the simple scenario of running a $2^{n}$ experiment in two blocks of size $2^{n-1}$. Here one would assign a blocking variable, say $\beta$, to one of the columns in the full factorial design matrix. This implies that the block is aliased with one of the effects and is said to be confounded with the effect. It is advisable to choose blocking variables using the principle of effect hierarchy. With this in mind, it makes sense to assign the blocking variable to the highest order interaction. This will cause the runs of the $2^{n}$ design to be grouped into two equally sized blocks of size $2^{n-1}$. One block would be run at the high level of $\beta$ and the other at the low level of $\beta$.

Example 2.3.1 Suppose we wished to run a $2^{3}$ design in two blocks. Keeping the effect hierarchy principle in mind, we could let $\beta=A B C$. This implies that blocks are confounded with the 3 fi $A B C$. We could assign the treatment combinations to block 1 whenever $A B C=-$ (that is, whenever the product $A B C=-1$ ) and assign the treatment combinations to block 2 whenever $A B C=+($ that is, whenever $A B C=1)$. We may summarize the assignment of the 8 runs to the two blocks with the following 
$2^{3}$ design matrix, sorted by block number:

$$
\left(\begin{array}{ccccc}
A & B & C & \beta=A B C & \text { Block } \\
- & - & - & - & 1 \\
+ & + & - & - & 1 \\
+ & - & + & - & 1 \\
- & + & + & - & 1 \\
+ & - & - & + & 2 \\
- & + & - & + & 2 \\
- & - & + & + & 2 \\
+ & + & + & + & 2
\end{array}\right) .
$$

If we wish to run an experiment in $2^{m}$ blocks we require $m$ blocking variables. To construct the blocking variables we must confound the blocks with multiple effect columns (from the full factorial design matrix) while using suitable replacement rules (Addelman, 1962). For example, to run an experiment in four blocks, we would use Table 2.1. Here, $\beta_{1}, \beta_{2}$ and $\beta_{1} \beta_{2}$ represent the three blocking variables, each assigned to a higher-order interaction. Note that the third blocking variable, $\beta_{1} \beta_{2}$, is automatically determined once $\beta_{1}$ and $\beta_{2}$ are chosen since we take $\beta_{1} \beta_{2}$ to be the product of $\beta_{1}$ and $\beta_{2}$.

Table 2.1: Running an Experiment in Four Blocks

\begin{tabular}{cccc}
\hline$\beta_{1}$ & $\beta_{2}$ & $\beta_{1} \beta_{2}$ & Block Indicator \\
\hline- & - & + & 1 \\
+ & - & - & 2 \\
- & + & - & 3 \\
+ & + & + & 4 \\
\hline
\end{tabular}

Other references detailing approaches to blocking $2^{n}$ designs include Kempthorne (1952), Anderson and McLean (1974) and Montgomery (2001). 
One model for a BFF design is

$$
y=f(\text { factor effects })+\epsilon+g(\text { block effects })+e,
$$

where $\epsilon$ and $e$ are the (mutually independent) factor and block effect error terms. For this model, the main assumption is that block-by-treatment interactions are negligible (Sitter et al., 1997 and Loeppky and Sitter, 2002).

For full factorial designs optimal blocking schemes may be obtained by applying the MA criterion to the block DCS (Sun, Wu and Chen, 1997). Sun et al. (1997) denote the number of $i$-factor interactions that are confounded with block effects by $A_{i}(b)$. Because no main effect should be confounded with block effects, $A_{1}(b)=0$ and the definition of $A_{i}(b)$ begins with $i=2$. Therefore, the WLP of the block DCS is given by

$$
W_{b}=\left(A_{2}(b), \ldots, A_{n}(b)\right) .
$$

Because $W_{b}$ is analogous to the WLP, W, for a FF design, we can apply the MA criterion to rank-order any two blocking schemes for $2^{n}$ full factorial designs.

Example 2.3.2 Suppose we wish to arrange a $2^{4}$ design in 4 blocks. Consider the blocking variables $\beta_{1}=A B$ and $\beta_{2}=A C$. The block $D C S$, denoted by $b_{1}$, is $I=$ $A B \beta_{1}=A C \beta_{2}=B C \beta_{1} \beta_{2}$. Consider an alternate blocking arrangement for the $2^{4}$ design given by $\beta_{1}=A B C$ and $\beta_{2}=C D$. The block $D C S, b_{2}$, is $I=A B C \beta_{1}=$ $C D \beta_{2}=A B D \beta_{1} \beta_{2}$. By simple application of the $M A$ criterion we see that $W_{b_{1}}=$ $(3,0,0)$ and $W_{b_{2}}=(1,2,0)$. Therefore, $b_{2}$ has less aberration than $b_{1}$ since $A_{2}\left(b_{2}\right)=$ $1<A_{2}\left(b_{1}\right)=3$.

\subsection{Blocked Two-level Fractional Factorial Designs}

The methodology by which one may construct optimal blocking schemes for $2^{n}$ designs has now been outlined. For $2^{n-k}$ FF designs, the issue of blocking is considerably more complicated due to the presence of two DCSs - the treatment DCS and the block DCS. The following example from Wu and Hamada (2000) illustrates how these two DCSs act jointly in determining the alias structure of factor effects and in determining the overall (combined) DCS of the design. 
Example 2.4.1 Consider the $2^{6-2} F F$ design in 4 blocks, with treatment $D C S, I=$ $A B C E=A B D F=C D E F$ and block $D C S I=A C D \beta_{1}=B C D \beta_{2}=A B \beta_{1} \beta_{2} . B y$ multiplying each word in the treatment $D C S$ by $A C D \beta_{1}$ we have $A C D \beta_{1}=B D E \beta_{1}=$ $B C F \beta_{1}=A E F \beta_{1}$. Therefore, the block effect $\beta_{1}$ is confounded with the four 3fi's, $A C D, B D E, B C F$ and $A E F$. Similarly, $B C D \beta_{2}=A D E \beta_{2}=A C F \beta_{2}=B E F \beta_{2}$ and $A B \beta_{1} \beta_{2}=C E \beta_{1} \beta_{2}=D F \beta_{1} \beta_{2}=A B C D E F \beta_{1} \beta_{2}$. The combined $D C S$ of the blocked $2^{6-2}$ FF designs is

$$
\begin{aligned}
I & =A B C E=A B D F=A C D \beta_{1}=B C D \beta_{2}=C D E F=B D E \beta_{1}=A D E \beta_{2} \\
& =B C F \beta_{1}=A C F \beta_{2}=A B \beta_{1} \beta_{2}=A E F \beta 1=B E F \beta 2=C E \beta_{1} \beta_{2} \\
& =D F \beta_{1} \beta_{2}=A B C D E F \beta_{1} \beta_{2} .
\end{aligned}
$$

Because of the presence of two distinct DCSs in $2^{n-k}$ BFF designs there is no clear choice for an extension of the MA criterion from the (blocked) $2^{n}$ full factorial design scenario. There have been recent attempts to address the problem by Bisgaard (1994a, b) and more thoroughly by Sitter et al. (1997).

Sitter et al. (1997) define the length of a word in the DCS of a $2^{n-k}$ BFF design to be

$$
\sharp c_{i}+(1.5) I_{\left[\sharp \beta_{i} \geq 1\right]},
$$

where $\sharp c_{i}$ and $\sharp \beta_{i}$ represent the number of factors and blocking variables in the word. If a given word contains at least one $\beta_{i}$ (i.e., $\sharp \beta_{i} \geq 1$ ), then the indicator variable, $I$, implies that the word length is increased by 1.5 . The motivation for introducing fractional word lengths is two-fold. First, Equation 2.6 implies that block main effects and block-by-block interactions contribute the same amount to the length of a word in a BFF design's DCS. Second, the choice of 1.5 results in agreement with the word ranking scheme $c c c<<c c b<<c c c c<<c c c b<<c c c c c<<\ldots$, where $c$ and $b$ denote factor and blocking variables, respectively, and $<<$ is interpreted as "less desirable than". Sitter et al. (1997) note that the choice of 1.5 is arbitrary. Any number between 1 and 2 would imply the previous ranking scheme. This definition of word length results in the WLP of a BFF design being of the form

$$
W=\left(A_{3}, A_{3.5}, \ldots, A_{n+1.5}\right),
$$

where $A_{i}$ denotes the number of words of length $i$ in the DCS, $3 \leq i \leq n+1.5$. 
Note that Equation 2.6 has now made it possible for a BFF design to have fractional resolution. Also, from Equation 2.7 it is evident that designs having words that alias or confound (factor or block) main effects with other main effects are not to be considered since $W$ allows only for those BFF designs that are of at least resolution 3 . The MA criterion can now be easily applied to a BFF design via sequential minimization of the WLP, $W$, in Equation 2.7 .

Example 2.4.2 To illustrate the above concepts we reproduce an example from Sitter et al. (1997). Consider blocking a $2^{8-2} F F$ design in 4 blocks. This implies that we require two blocking variables, $\beta_{1}$ and $\beta_{2}$. If the factor and blocking variable generators are chosen to be $G=A B C D, H=A B E F, \beta_{1}=A C E$ and $\beta_{2}=B D F$ then the $D C S$ will be given by

$$
\begin{aligned}
I & =A B C D G=A B E F H=A C E \beta_{1}=B D F \beta_{2}=C D E F G H=A B C D E F \beta_{1} \beta_{2} \\
& =B D E G \beta_{1}=B C F H \beta_{1}=A C F G \beta_{2}=A D E H \beta_{2}=E G F \beta_{1} \beta_{2} \\
& =C D H \beta_{1} \beta_{2}=A D F G H \beta_{1}=B C E G H \beta_{2}=A B G H \beta_{1} \beta_{2} .
\end{aligned}
$$

This design has (fractional) resolution 4.5 with $W L P, W=(0,0,0,4,2,5,1,2,0,1)$.

Another optimality criterion given considerable attention in the literature when rank-ordering factorial designs is the total number of clear effects that a design possesses. A main effect or $2 \mathrm{fi}$ is said to be clear if it is not aliased (or confounded) with any other main effects, 2fi's or block effects. Sun et al. (1997) rank-ordered both blocked $2^{n}$ full factorial and $2^{n-k}$ BFF designs according to this criterion. It has been observed that these optimality criteria (MA and total number of clear effects) may lead to different "optimal" designs (Sitter et al., 1997).

\subsection{Split-plot Designs}

Split-plot designs arise in experimentation when randomization restrictions exist while assigning treatments to experimental units. These situations occur primarily in agricultural and industrial applications but are being observed in an increasing number of research areas in recent years (Silverstein, 1985; Algina, 1997). Kempthorne's (1952) overview of the split-plot framework came at a time when the split-plot approach in experimental design was becoming popular among design practitioners. 
The split-plot designs described by Kempthorne (1952) were originally motivated by agricultural experiments with the whole-plots (WPs) usually being large areas of land and the sub-plots (SPs) being smaller portions of land within the larger areas (see also, Yates, 1935). In recent years, authors such as Box and Jones (1992), Bingham and Sitter (2001) and Montgomery (2001) have investigated the use of split-plot designs within industry.

To motivate further theoretical developments we will first look at a small splitplot experimental design. The example is taken largely from Milliken and Johnson (1992).

Example 2.5.1 Consider a simple split-plot experimental design involving just two multi-level factors, say $A$ and $B$. The SP factor, say $B$, and its b levels are completely randomized to the SPs within the WP factor $A$. That is, for each level of $A$ we have a grouping of the $b$ levels of $B$. The number of WPs is a multiple of a, the number of levels of the WP factor $A$.

Suppose factor $A$ has four levels, factor $B$ has three levels and there are eight WPs each consisting of three SP experimental units. The levels of $A$ are randomly assigned to the WPs and the levels of $B$ are subsequently randomly assigned to the SPs, within the WPs, see Figure 2.2. The randomization restriction is that only one WP treatment combination takes place on each WP whereas all three SP treatment levels take place within a given WP.

Figure 2.2: A Simple Split-plot Experiment.

\begin{tabular}{|c|c|c|c|c|c|c|c|}
\multicolumn{1}{c}{$A_{3}$} & $A_{1}$ & $A_{2}$ & \multicolumn{1}{c}{$A_{4}$} & $A_{3}$ & $A_{4}$ & $A_{1}$ & $A_{2}$ \\
\hline$B_{2}$ & $B_{1}$ & $B_{2}$ & $B_{3}$ & $B_{1}$ & $B_{1}$ & $B_{3}$ & $B_{2}$ \\
\hline$B_{1}$ & $B_{2}$ & $B_{3}$ & $B_{2}$ & $B_{3}$ & $B_{2}$ & $B_{1}$ & $B_{3}$ \\
\hline$B_{3}$ & $B_{3}$ & $B_{1}$ & $B_{1}$ & $B_{2}$ & $B_{3}$ & $B_{2}$ & $B_{1}$ \\
\hline
\end{tabular}

The larger WP experimental units are expected to be more heterogeneous "across" than "within". Consequently, the between WP variability is expected to be larger than the within WP variability and we write

$$
\sigma_{W P}^{2}>\sigma_{S P}^{2}
$$


Also, since the WP treatments in a split-plot design are confounded with the WPs and the SP treatments are not, it is wise to assign the factors that we are most interested in to the SPs, if at all possible.

The model for the split-plot experiment in Example 2.5.1 is given by

$$
y_{i j k}=\mu+A_{i}+e_{i j}+B_{k}+(A B)_{i k}+\epsilon_{i j k},
$$

where $i=1,2,3,4 ; j=1,2 ; k=1,2,3$. The WP portion of the model is represented by $\mu+A_{i}+e_{i j}$ and $B_{k}+(A B)_{i k}+\epsilon_{i j k}$ represents the SP model portion. Note that in a split-plot design there are two error terms in the model-one for the WPs, $e_{i j}$, and one for the SPs, $\epsilon_{i j k}$. Also, it is assumed that

$$
e_{i j} \backsim N\left(0, \sigma_{W P}^{2}\right)
$$

and

$$
\epsilon_{i j k} \backsim N\left(0, \sigma_{S P}^{2}\right) .
$$

Moreover, we assume all of the $e_{i j}$ and $\epsilon_{i j k}$ are mutually independent. The structure of the analysis of variance (ANOVA) table for the split-plot design in Example 2.5.1 is given in Table 2.2.

Table 2.2: Stucture of the ANOVA Table for the Two-factor Split-plot Experiment in Example 2.5.1.

\begin{tabular}{lcc}
\hline \hline Source of Variation & df \\
\hline WP & & \\
& A & 3 \\
& Error (WP) & 4 \\
SP & & \\
& B & 2 \\
& AB & 6 \\
& Error (SP) & 8 \\
\hline Total & & 23 \\
\hline
\end{tabular}

Since there are larger numbers of degrees of freedom (df) for SP error than there are for WP error, and because the SP error is likely smaller than the WP error, 
we will typically have greater power for detecting statistically significant SP effects. This greater power results because we have, in essence, more observations of the individual SP treatments than we do for the WP treatments. This in conjunction with the expectation that SP experimental units are more homogeneous than WP units should motivate one to assign the factors of most interest to the SP units if at all possible.

\subsection{Two-level Fractional Factorial Split-plot De- signs}

Some characteristics of a simple split-plot design were outlined in the previous section. At that point we did not set restrictions on the number of levels that the factors possessed. We now turn our attention to factors that have only two levels. By doing this we will be able to specifically consider $2^{n-k} \mathrm{FF}$ designs with a split-plot structure.

\subsubsection{Full Factorial Split-plot Designs}

Suppose the initial intent was to run a $2^{n}$ design, but upon further consideration $n_{1}$ of the factors were deemed hard-to-vary. To save resources (money, time or labour) one can randomly choose one of the treatment combinations of the $n_{1}$ difficult-to-vary factors and then run all of the level combinations of the $n_{2}$ remaining easy-to-vary factors in random order-this is done while holding the $n_{1}$ factors fixed. In these cases, we have randomization restrictions in the design of the experiment which results in a split-plot structure.

For a $2^{n}$ design with $n_{1}$ WP (hard-to-vary) factors and $n_{2}$ SP (easy-to-vary) factors we are now faced with a full factorial split-plot design. The design matrix for the $2^{n}=2^{n_{1}+n_{2}}$ full factorial split-plot design is identical to that of the $2^{n}$ full factorial design. The only difference is the order in which the runs are performed.

\subsubsection{Fractional Factorial Split-plot Designs}

Two FFs underlie the structure of an FFSP design - the $n_{1}$ WP or hard-to-vary factors arranged as a $2^{n_{1}-k_{1}} \mathrm{FF}$ and the $n_{2} \mathrm{SP}$ or easy-to-vary factors arranged as a 
$2^{n_{2}-k_{2}} \mathrm{FF}$. However, if one views the WP and SP designs separately, there are limited design options (Bingham and Sitter, 1999a). Rather, it is better if WP factors are included in the SP factor generators (Kempthorne, 1952, p. 318). This approach often allows one to increase the resolution of an FFSP design. A two-level FFSP design is typically denoted by $2^{\left(n_{1}+n_{2}\right)-\left(k_{1}+k_{2}\right)}$; this notation parallels that of Huang, Chen and Voelkel (1998) and Bingham and Sitter (1999a, 2001) and implies that there are $n=n_{1}+n_{2}$ treatment factors, $k=k_{1}+k_{2}$ added factors and $n_{1}+n_{2}-k_{1}-k_{2}$ basic factors from which the added factors are generated.

Example 2.6.1 Consider a $2^{(3+3)-(1+1)}$ FFSP design. This design contains three $W P$ factors $(A, B$ and $C$ ) and three SP factors ( $p, q$ and $r$ ). (We will consistently denote $W P$ and $S P$ factors with uppercase and lowercase letters, respectively.) It is implicitly assumed that it is difficult to change the levels of $A, B$ and $C$, thus their WP designation.

Suppose that $C=A B$ and $r=A p q$ are the design generators. The DCS of the FFSP design is

$$
I=A B C=A p q r=B C p q r
$$

and is of resolution 3. The design matrix is given in Figure 2.3. Note that the WP level of the design is a $2^{3-1} F F$, thus there are only 4 distinct WP level combinations within the 16-run design matrix. The 4 level combinations of the easy-to-vary factors are run within each WP treatment combination, thus creating the split-plot structure.

The FFSP setting may be modelled as

$$
y=f(\mathrm{WP} \text { effects })+e+g(\mathrm{SP} \text { effects })+\epsilon,
$$

(Bingham and Sitter, 2001). The WP and SP error terms are $e$ and $\epsilon$, respectively, where it is assumed that $e$ and $\epsilon$ are mutually independent normal random errors. That is, we have $e \backsim N\left(0, \sigma_{W P}^{2}\right)$ and $\epsilon \backsim N\left(0, \sigma_{S P}^{2}\right)$, as in Section 2.5. Lastly, $f(\cdot)$ and $g(\cdot)$ are functions of the WP and SP factorial effects.

\subsubsection{Minimum Aberration and Rank-ordering FFSP De- signs}

Huang et al. (1998) extended the concept of MA to FFSP designs. 
Figure 2.3: A $2^{(3+3)-(1+1)}$ FFSP Design.

$$
\begin{aligned}
& \begin{array}{lllllll}
A & B & C & p & q & r
\end{array} \\
& \left(\begin{array}{ccccc}
- & - & - & - & - \\
& + & - & + \\
& - & + & + \\
& + & + & - \\
+ & - & - & - & + \\
& + & - & - \\
& - & + & - \\
& + & + & + \\
- & - & - & - \\
& + & - & + \\
& - & + & + \\
+ & + & - \\
& - & - & + \\
+ & - & - \\
- & + & - \\
+ & + & +
\end{array}\right) .
\end{aligned}
$$

Definition 2.6.1 (MA FFSP Design) Suppose that $D_{1}$ and $D_{2}$ are two $2^{\left(n_{1}+n_{2}\right)-\left(k_{1}+k_{2}\right)}$ FFSP designs. Let $r$ be the smallest $i$ such that $A_{i}\left(D_{1}\right) \neq A_{i}\left(D_{2}\right)$. Then $D_{1}$ is said to have less aberration than $D_{2}$ if $A_{r}\left(D_{1}\right)<A_{r}\left(D_{2}\right)$. If no such $i$ exists, then $D_{1}$ and $D_{2}$ are said to have equal aberration. A FFSP design is said to be a MA FFSP design if no other FFSP design has less aberration.

Huang et al. (1998) proposed two methods for finding MA FFSP designs and subsequently constructed an extensive catalogue of MA FFSP designs. However, as they noted, their catalogue is incomplete. As an improvement to the existing MA FFSP design search techniques, Bingham and Sitter (1999a) introduced a more efficient algorithm that constructed the set of all nonisomorphic (NI) two-level FFSP designs. (Two designs, $D_{1}$ and $D_{2}$ are said to be NI if one cannot obtain $D_{2}$ from 
$D_{1}$ by relabeling the factors of $D_{1}$.) From this set of NI designs, Bingham and Sitter (1999a) applied the MA criterion in Definition 2.6.1 to find the set of all NI 8, 16 and 32-run MA FFSP designs containing up to 7,15 and 10 factors, respectively.

For fixed $n_{1}, n_{2}, k_{1}$ and $k_{2}$ it turns out there may be many NI MA FFSP designs (Bingham and Sitter, 1999a). Bingham and Sitter (2001) describe how one can choose between NI MA FFSP designs by considering which effects are compared to which variance components. They base their selection procedure on work done by Bisgaard (2000). Bisgaard (2000) showed that confusion regarding which error terms are to be used when assessing the statistical significance of estimated split-plot effects could be eliminated by considering the following rules (as summarized by Bingham and Sitter, 2001):

1. WP main effects and interactions involving only WP factors are compared to the WP error.

2. SP main effects and interactions involving at least one SP factor that are aliased with either WP main effects or interactions involving only WP factors are compared to the WP error.

3. SP main effects and interactions involving at least one SP factor that are not aliased with either WP main effects or interactions involving only WP factors are compared to the SP error.

Since there is typically greater power in detecting statistically significant SP effects than WP effects, we wish to have as many SP effects as possible tested against the SP error. Bingham and Sitter (2001) provide tables of 16 and 32-run NI FFSP designs ranked according to the MA criterion and by the total number of SP effects that are tested against the SP error term. This procedure provides two criteria for ranking "optimal" FFSP designs. 


\section{Chapter 3}

\section{BFFSP Designs and MA}

Chapter 2 detailed the motivation (as well as the approach) for blocking two-level full factorial and FF designs. In Chapter 2 we also provided a detailed introduction to FFSP designs. For each design scenario in Chapter 2 we specifically considered the MA optimality criterion for ranking the "goodness" of designs. This chapter extends the material in Chapter 2 by considering the presence of blocking in two-level FFSP designs. We also provide a straightforward extension of the MA criterion to the BFFSP design setting. Our approach is based upon that of Sitter et al. (1997) and enables a practitioner to select "good" BFFSP designs for the estimation of (WP and SP) main effects and 2fi's.

\subsection{Three Approaches to Constructing Blocking Variables}

The motivation for blocking a FFSP design parallels the discussion in Chapter 2 where we considered blocking a full factorial design. If it is thought that experimental conditions will not remain homogeneous for all $2^{\left(n_{1}+n_{2}\right)-\left(k_{1}+k_{2}\right)}$ runs of the FFSP design, blocking may represent a practical design option. For example, if a single batch of raw material is not large enough to permit all $2^{\left(n_{1}+n_{2}\right)-\left(k_{1}+k_{2}\right)}$ treatment combinations to be run, then blocking may be used to account for variability between batches. Industrial experiments may also be blocked using specific time periods (for example, blocked by week). This enables researchers to account for variability that occurs in a process over time, including variability due to changing environmental 
conditions or personnel.

We will see in this chapter that blocking FFSP designs is complicated by the presence of the two FFs underlying the split-plot structure. Moreover, we will demonstrate that there is more than one way to block a FFSP design. We will show that one may induce blocking at the WP level using three distinct, yet related, approaches. The first approach, pure WP blocking, requires that blocking variables be generated exclusively by WP factors. The second approach, which we call "separation", uses blocking generators that consist of SP factors alone, or SP factors in conjunction with WP factors. The third approach, mixed blocking, incorporates properties of both pure WP blocking and separation.

\subsubsection{Pure Whole-Plot Blocking}

Pure WP blocking requires that blocking variables be generated exclusively by WP factors. However, this does not imply that the SP factors do not play a role in the formation of the WP blocking variables. Rather, since factor generators and blocking generators are formed simultaneously, the amount of fractionation at the SP level will impact the selection of blocking generators.

For pure WP blocking, the $i^{t h}$ pure WP blocking variable is denoted by $\beta_{i}, i=$ $1, \ldots, b_{1}$. In each block, $2^{n_{1}-k_{1}-b_{1}}$ distinct WP treatment combinations are present, $1 \leq b_{1} \leq n_{1}-k_{1}-1$. (The rationale for the upper bound on the allowable number of WP blocking factors is discussed in Section 3.2.2.) Associated with each WP treatment combination are $2^{n_{2}-k_{2}}$ SP treatment combinations. Therefore, if a design possesses $b_{1}$ WP blocking variables, the treatment combinations are grouped into $2^{b_{1}}$ WP blocks of size $2^{\left(n_{1}+n_{2}\right)-\left(k_{1}+k_{2}\right)-b_{1}}$.

For compactness of notation, it is useful to incorporate $b_{1}$, the number of pure WP blocking variables, into the exponent of $2^{\left(n_{1}+n_{2}\right)-\left(k_{1}+k_{2}\right)}$. One such representation is given by $2^{\left(n_{1}+n_{2}\right)-\left(k_{1}+k_{2}\right) \pm\left(b_{1}+0\right)}$. The rationale for this notation, including the presence of the zero in the exponent, will be given in Sections 3.1.2 and 3.1.3.

Example 3.1.1 Suppose that we wish to run a $2^{(3+3)-(0+1)}$ FFSP design in $2^{1}=2$ blocks. In each block there will be four distinct WP treatment combinations and, corresponding to each of the WP treatment combinations, there will be four SP treatment combinations. Thus there will be 16 runs per block. 
Figure 3.1: Geometric Representation of the $2^{(3+3)-(0+1) \pm(1+0)}$ BFFSP Design. The eight corners of the larger cube represent the eight treatment combinations of the WP factors $A, B$ and $C$. Associated with each WP treatment combination are four treatment combinations, denoted by circles, of the SP factors, $p, q$ and $r$. Circles of similar color signify treatment combinations belonging to the same block.

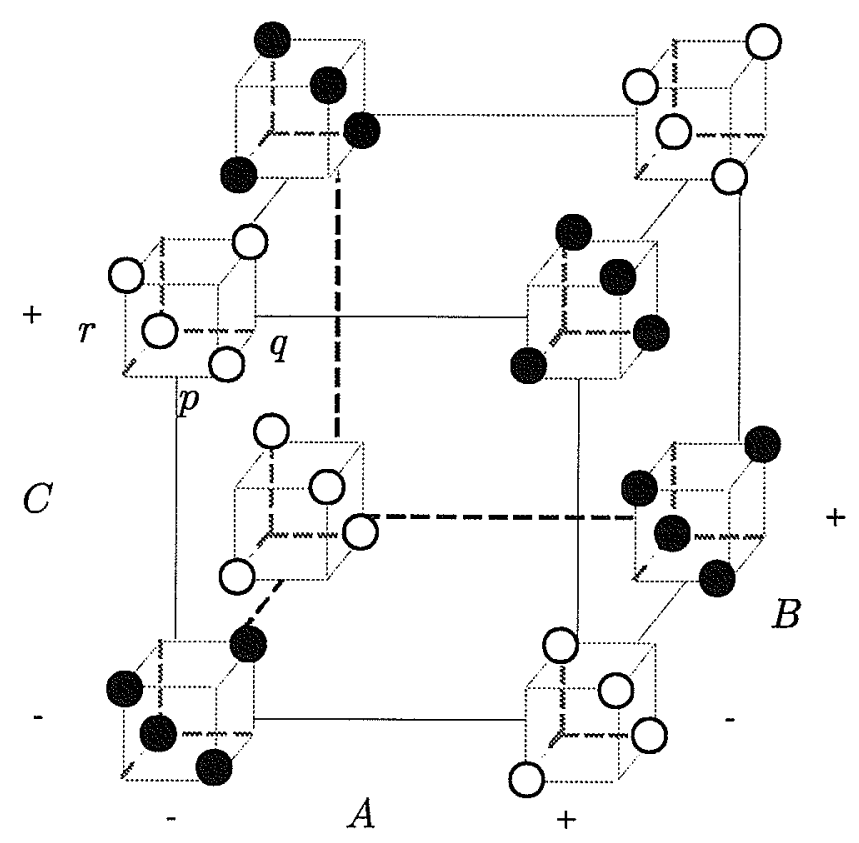

One possible $2^{(3+3)-(0+1) \pm(1+0)}$ BFFSP design is constructed by using $\beta_{1}=A B C$ as the pure $W P$ blocking generator and $r=A B p q$ as the $S P$ factor generator. The resulting defining contrast subgroup (DCS) is $I=A B C \beta_{1}=A B p q r=C p q r \beta_{1}$. Figure 3.1 provides a geometric representation of the 32 runs of this BFFSP design. (The standard run order of the $2^{(3+3)-(0+1) \pm(1+0)}$ design is given in Appendix A.) The eight corners of the large cube in Figure 3.1 represent all possible WP level combinations. Associated with each WP level combination are four SP level combinations, represented by the circles on the corners of the smaller cubes. Note that there are only 
four SP treatment combinations associated with each WP treatment combination due to the fractionation at the SP level; recall, $r=A B p q$. In Figure 3.1, circles of similar color (black or white) represent treatment combinations run within the same block.

\subsubsection{Separation}

It is useful to recognize that blocking variables need not be generated solely by WP factors. In fact, blocking variables may be generated by exclusively using SP factors or by using SP factors in tandem with WP factors in blocking generators. We call this method by which one includes SP and (possibly) WP factors in the blocking generators to be separation, and the blocking variables formed in this manner to be separators. With this definition, note that each separator must contain at least one SP factor and may or may not contain any WP factors. To ensure that blocking at the WP level is preserved, we envision that one would conduct the experiment by first performing the runs in block 1 , and then block 2, and so on-over the whole experiment-not just at the SP level. Some of the runs that, without blocking, would have been near to each other (because they were in the same WP) are now separated in time (if blocking is being done in time).

In this form of blocking, the $j^{\text {th }}$ separator is denoted by $\delta_{j}, j=1, \ldots, b_{2}$. In each block, $2^{n_{1}-k_{1}}$ WP treatment combinations are present, and associated with each WP treatment combination are $2^{n_{2}-k_{2}-b_{2}}$ SP treatment combinations, $1 \leq b_{2} \leq n_{2}-k_{2}-1$. (The rationale for the upper bound on the number of separators is discussed in Section 3.2.2.) The preceding formulae exemplify one of the consequences of separation. Specifically, the process of separation achieves pseudo-replication at the WP level at the expense of further fractionation at the SP level (Brewster and McLeod, 2000). This pseudo-replication occurs since each separator results in a doubling of the number of runs at the WP level. The "pseudo" aspect of this replication process arises since, for a given replicated WP treatment combination, the associated SP treatment combinations may be different. In any case, separation causes increased precision for estimates of WP effects. We see these precision gains by first recalling that there are $2^{n_{1}-k_{1}}$ WP runs per block. Therefore, for $2^{b_{2}}$ blocks, we will, over the course of the experiment, have reset the WP treatment combinations $2^{b_{2}} 2^{n_{1}-k_{1}}=2^{n_{1}-k_{1}+b_{2}}$ times. At the SP level, each additional separator causes a decrease, by a factor of two, in the number of SP treatment combinations associated with each WP treatment 
combination, when compared to the pure WP blocking approach. Central to this concept of pseudo-replication is the recognition that, at the WP level, the appropriate analysis of a split-plot (or FFSP) design begins by averaging the responses over the SPs contained within each WP. The use of the term pseudo-replication reflects that, although we have replicated the treatment combinations for the WP factors, the associated treatment combinations for the SP factors are not the same.

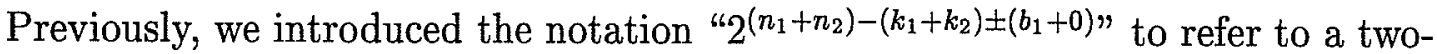
level BFFSP design having $b_{1}$ pure WP blocking variables. This notation signified that blocking was accomplished exclusively through the use of WP factors. When performing blocking via separation we refer to the BFFSP design with no WP blocking variables and $b_{2}$ separators as a $2^{\left(n_{1}+n_{2}\right)-\left(k_{1}+k_{2}\right) \pm\left(0+b_{2}\right)}$ BFFSP design. Note that the \pm symbol was chosen in part to remind the practitioner that separators affect the way that we think about the amount of fractionation and replication in the design, although they do not affect the total number of runs.

Example 3.1.2 To illustrate the process of separation we return to the $2^{(3+3)-(0+1)}$ FFSP design in Example 3.1.1. Again we wish to group the 32-run design into two blocks; however, here we will accomplish this by using one separator instead of one pure WP blocking variable.

One possible $2^{(3+3)-(0+1) \pm(0+1)}$ BFFSP design is formed by using $\delta_{1}=A B q$ and $r=A B C p$ as the separator and SP factor generator, respectively. The DCS is given by $I=A B C p r=A B q \delta_{1}=C p q r \delta_{1}$. The use of WP factors ( $A$ and $B$ ) and a single $S P$ factor $(q)$ is demonstrated in the formation of $\delta_{1}$. (Other generators for $\delta_{1}$ are of course possible. The selection, $\delta_{1}=A B q$, was simply made to exemplify the properties of separator generators.)

Figure 3.2 provides a geometric representation of the 32 runs of the $2^{(3+3)-(0+1) \pm(0+1)}$ BFFSP design. (The standard run order of the $2^{(3+3)-(0+1) \pm(0+1)}$ design is given in Appendix A.) As in Figure 3.1, the eight corners of the large cube in Figure 3.2 represent all possible WP level combinations. Associated with each WP level combination are four SP level combinations, represented by the circles on the corners of the smaller cubes. Note that there are only four SP treatment combinations associated with each WP treatment combination due to the fractionation at the SP level; recall, $r=A B C p$. In Figure 3.2, circles of similar color (black or white) represent treatment combinations run within the same block. Observe that all eight possible 
Figure 3.2: Geometric Representation of the $2^{(3+3)-(0+1) \pm(0+1)}$ BFFSP Design. The eight corners of the larger cube represent the eight treatment combinations of the WP factors $A, B$ and $C$. Associated with each WP treatment combination are four treatment combinations, denoted by circles, of the SP factors, $p, q$ and $r$. Circles of similar color signify treatment combinations belonging to the same block.

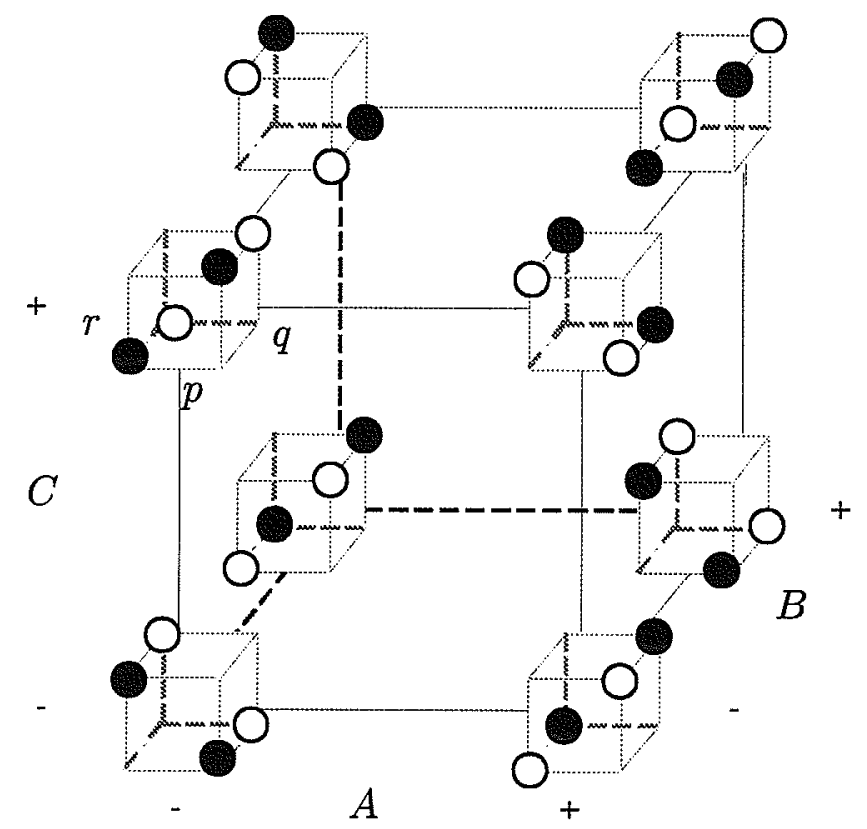

WP treatment combinations are present within each block, in contrast to the design in Example 3.1.1 where there were only four distinct WP treatment combinations per block. As a result of separation we have thus seen a two-fold increase in the number of distinct WP treatment combinations in each block of the design. Conversely, within each block each WP treatment combination has only two associated SP treatment combinations, as compared to four, in Example 3.1.1. This demonstrates the further fractionation that always occurs at the SP level when separation has taken place. 


\subsubsection{Mixed Blocking}

Mixed blocking is a natural extension of the previous two blocking methods in that we now simultaneously use pure WP blocking variables and separators. Again the $i^{\text {th }}$ pure WP blocking variable and the $j^{\text {th }}$ separator are denoted by $\beta_{i}$ and $\delta_{j}$. Under mixed blocking, the formation of $b_{1}$ pure WP blocking variables and $b_{2}$ separators causes the subsequent $2^{\left(n_{1}+n_{2}\right)-\left(k_{1}+k_{2}\right) \pm\left(b_{1}+b_{2}\right)}$ BFFSP design to be run in $2^{b_{1}+b_{2}}$ blocks, where $1 \leq b_{1} \leq n_{1}-k_{1}-1$ and $1 \leq b_{2} \leq n_{2}-k_{2}-1$. Each block will contain $2^{n_{1}-k_{1}-b_{1}}$ WP treatment combinations and associated with each distinct WP treatment combination will be $2^{n_{2}-k_{2}-b_{2}}$ SP treatment combinations, for a total of $\left(2^{b_{1}+b_{2}}\right)\left(2^{n_{1}-k_{1}-b_{1}}\right)\left(2^{n_{2}-k_{2}-b_{2}}\right)=2^{\left(n_{1}+n_{2}\right)-\left(k_{1}+k_{2}\right)}$ runs. Therefore, as expected, the total number of runs remains unchanged under mixed blocking.

Example 3.1.3 Suppose we wish to run a $2^{(3+3)-(0+1)}$ design in four blocks by using both a pure WP blocking variable, $\beta_{1}$, and a separator, $\delta_{1}$. One possible $2^{(3+3)-(0+1) \pm(1+1)}$ $B F F S P$ design is formed by using $r=A B q$ as the $S P$ factor generator and $\beta_{1}=A B C$ and $\delta_{1}=A C p r$ as the pure WP blocking generator and separator, respectively. The $D C S$ is given by $I=A B q r=A B C \beta_{1}=A C p r \delta_{1}=C q r \beta_{1}=B C p q \delta_{1}=B p r \beta_{1} \delta_{1}=$ $A p q \beta_{1} \delta_{1}$.

Figure 3.3 provides a geometric representation of the 32 runs of the $2^{(3+3)-(0+1) \pm(1+1)}$ BFFSP design. (The standard run order of the $2^{(3+3)-(0+1) \pm(1+1)}$ design is given in Appendix A.) Each block contains eight runs (circles having identical form represent treatment combinations run within the same block) where each of the four distinct WP treatment combinations within a block have two associated SP treatment combinations. Note that the attributes of separation carry directly over to the mixed blocking scenario. By this we mean that, as a consequence of the separator, we again obtain increased replication of the WP treatment combinations at the expense of further fractionation at the SP level.

\subsection{MA for BFFSP Designs}

To be able to provide a formal definition of what is meant by a MA BFFSP design, we extend several concepts first introduced in Chapter 2. Recall that Sitter et al. 
Figure 3.3: Geometric Representation of the $2^{(3+3)-(0+1) \pm(1+1)}$ BFFSP Design. The eight corners of the larger cube represent the eight treatment combinations of the WP factors $A, B$ and $C$. Associated with each WP treatment combination are four treatment combinations, denoted by circles, of the SP factors, $p, q$ and $r$. Circles having identical form denote treatment combinations belonging to the same block.

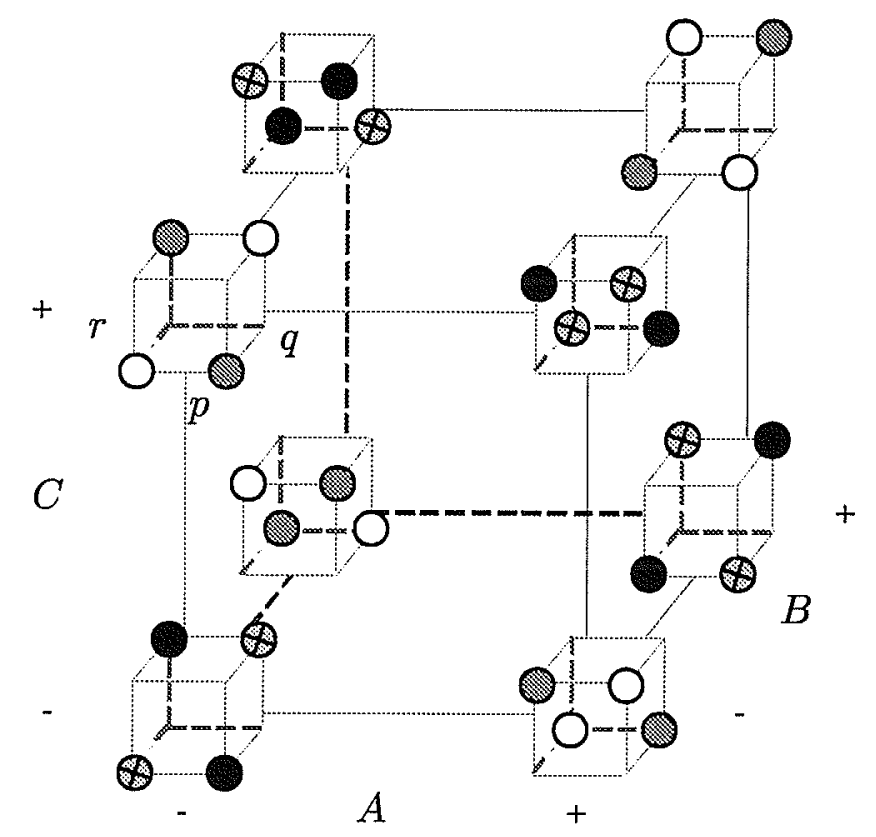

(1997) define the length of a word in the DCS of a $2^{n-k}$ BFF design to be

$$
\sharp c_{i}+(1.5) I_{\left[\sharp \beta_{i} \geq 1\right]},
$$

where $\sharp c_{i}$ and $\sharp \beta_{i}$ represent the number of factors and blocking variables in the word. The motivation for introducing fractional word lengths was two-fold. First, recall that Equation 3.1 implies that block main effects and block-by-block interactions contribute the same amount to the length of a word in a BFF design's DCS. Second, the choice of 1.5 results in agreement with the word ranking scheme $c c c<<c c b<<$ 
$c c c c<<c c c b<<c c c c c<<\ldots$, where $<<$ is interpreted as "less desirable than". This definition of word length resulted in the WLP of a BFF design being of the form

$$
W=\left(A_{3}, A_{3.5}, \ldots, A_{n+1.5}\right),
$$

where $A_{i}$ denotes the number of words of length $i$ in the DCS, $3 \leq i \leq n+1.5$.

By extension we define the length of a word in the DCS of a $2^{\left(n_{1}+n_{2}\right)-\left(k_{1}+k_{2}\right) \pm\left(b_{1}+b_{2}\right)}$ BFFSP design to be

$$
\sharp c_{i}+(1.5) I_{\left[\sharp\left(\beta_{i}+\delta_{j}\right) \geq 1\right]},
$$

where $\sharp c_{i}$ and $\sharp\left(\beta_{i}+\delta_{j}\right)$ represent the number of factors and blocking variables in the word. If in a given word we have that $\sharp\left(\beta_{i}+\delta_{j}\right) \geq 1$ then the indicator variable, $I$, implies that the word length must be increased by 1.5. Equation 3.3 causes the WLP of a BFFSP design to be of the form

$$
W=\left(A_{3}, A_{3.5}, \ldots, A_{n_{1}+n_{2}+1.5}\right),
$$

where $A_{i}$ signifies the number of words of length $i$ in the DCS, $3 \leq i \leq n_{1}+n_{2}+1.5$.

Example 3.2.1 Consider the $2^{(3+3)-(0+1) \pm(1+1)}$ BFFSP design given in Example 3.1.3. The DCS of the design is $I=A B q r=A B C \beta_{1}=A C p r \delta_{1}=C q r \beta_{1}=B C p q \delta_{1}=$ $B p r \beta_{1} \delta_{1}=A p q \beta_{1} \delta_{1}$, which yields the WLP, $W=(0,0,1,4,0,2)$. Note that for the sake of brevity we often truncate the vector $W$ at the largest value of $i$ for which $A_{i} \neq 0$. In this example, the vector $W$ is thus truncated at $A_{5.5}=2$.

The MA criterion, applied under the word length definition given in Equation 3.3, amounts to the sequential minimization of $A_{3}, A_{3.5}, A_{4}, A_{4.5}, A_{5}, A_{5.5}, A_{6}, \ldots$ From this observation, we may extend the MA criterion to the BFFSP design setting.

Definition 3.2.1 (MA BFFSP Design) Suppose that $D_{1}$ and $D_{2}$ are two $2^{\left(n_{1}+n_{2}\right)-\left(k_{1}+k_{2}\right) \pm\left(b_{1}+b_{2}\right)}$ BFFSP designs. Let $r$ be the smallest $i$ such that $A_{i}\left(D_{1}\right) \neq$ $A_{i}\left(D_{2}\right), 3 \leq i \leq n_{1}+n_{2}+1.5$. Then $D_{1}$ is said to have less aberration than $D_{2}$ if $A_{r}\left(D_{1}\right)<A_{r}\left(D_{2}\right)$. If no such $i$ exists, then $D_{1}$ and $D_{2}$ are said to have equal aberration. A BFFSP design is said to be a MA BFFSP design if no other BFFSP design has less aberration. 


\subsubsection{A Limitation on the Word Length Definition}

According to Chen and Cheng (1999), the word length definition (Equation 3.1) of Sitter et al. (1997) has some limitations. They argue that words of length 6 should be less desirable than words of length 5.5, even though the definition of word length puts these words in the opposite order. Note that, if $A_{6} \neq 0$, then a number of 3fi's are aliased with other 3fi's; whereas, if $A_{5.5} \neq 0$, then at least one (less important) $4 \mathrm{fi}$ is confounded with blocks.

Since our definition of word length (Equation 3.3) parallels that of Sitter et al. (1997), it suffers from the same limitations. However, as our focus is on the estimation of main effects and 2fi's, the definition remains a useful measure for assessing the estimation capacity of BFFSP designs. As we shall see, in the catalog of MA BFFSP designs presented in Chapter 5, all of the designs are of resolution 4.5 or less. Hence, the issue raised by Chen and Cheng (1999) will not play any role, at least as far as the shortest word in the DCS is concerned.

\subsubsection{Ineligible and Impractical Designs}

Designs with resolution $R<3$ are deemed ineligible and will not be considered. This justifies the restriction $1 \leq b_{1} \leq n_{1}-k_{1}-1$ (Section 3.1.1), since if one generates $b_{1}$ WP blocking variables where $b_{1}$ is greater than the upper bound, one is guaranteed to obtain $R<3$. In this case, the number of WP blocking variables is greater than or equal to the number of WP basic factors.

There exist BFFSP designs having $R \geq 3$ yet are "impractical" to run. By an impractical design we refer to a BFFSP design having as many distinct WP treatment combinations per block as there are runs in the block. Clearly, if we are to change the levels of the hard-to-vary WP factors for each experimental run we are defeating the purpose of running the split-plot design in the first place.

Note that any BFFSP design with $b_{2}=n_{2}-k_{2}$ (one more than the upper bound for $b_{2}$; Section 3.1.2) will have as many distinct WP treatment combinations per block as there are runs in a block. That is, any design with $b_{2}=n_{2}-k_{2}$ will be impractical. This notion of impracticality can be seen from the following vantage point. Recall that for each additional separator there is a decrease, by a factor of two, in the number of SP treatment combinations associated with each WP treatment combination while 
a two-fold increase in the number of distinct WP runs is realized. This relationship may be represented notationally by $2^{\left(b_{2}+n_{1}-k_{1}\right)+\left(n_{2}-k_{2}-b_{2}\right)}$. Therefore, if $b_{2}=n_{2}-k_{2}$, the SP portion of the design in essence "disappears", requiring us to reset the WP factor level combinations at each run.

Table A.5 in Appendix A provides the standard run order of an impractical $2^{(4+2)-(0+1) \pm(2+1)}$ BFFSP design, where $b_{2}=n_{2}-k_{2}=1$. It is clear from Table A.5 that at least one WP factor must be reset for each run, thus destroying the split-plot nature of the design. 


\section{Chapter 4}

\section{Variance Forms for Split-plot Effects}

In the analysis of designed experiments the error structure of the estimated factorial effects (contrasts) should be considered by the experimenter. One may argue that knowledge of the variance structure for the effects is especially critical in the analysis of split-plot designs since WP and SP effects have different error structures due to randomization restrictions. When testing for the significance of an estimated effect one must test the given effect against the appropriate variance. If an incorrect variance is used, erroneous inferences regarding the significance of that effect may occur.

In this chapter, the variance forms for split-plot effects arising from the three blocking approaches (Chapter 3) are constructed and compared. This research extends the methods of Bisgaard (2000) who provided general results for $2^{\left(n_{1}+n_{2}\right)-\left(k_{1}+k_{2}\right)}$ FFSP designs. As discussed in Chapter 2, Bingham and Sitter (2001) summarize the results of Bisgaard (2000) as follows:

1. WP main effects and interactions involving only WP factors are compared to the WP error.

2. SP main effects and interactions involving at least one SP factor that are aliased with either WP main effects or interactions involving only WP factors are compared to the WP error.

3. SP main effects and interactions involving at least one SP factor that are not 
aliased with either WP main effects or interactions involving only WP factors are compared to the SP error.

\subsection{Derivation of Effect Variances}

Again, the intent of this chapter is to investigate the error structure of WP and SP contrasts in BFFSP designs. To thoroughly demonstrate the construction of these effect variances we need to consider the following four scenarios:

1. FFSP designs (as investigated by Bisgaard (2000)).

2. BFFSP designs with pure WP blocking.

3. BFFSP designs with blocking via separation.

4. BFFSP designs with mixed blocking.

\subsubsection{Variance Forms for FFSP Designs}

The results in this section are a summary of the work done by Bisgaard (2000) in the FFSP design setting. However, some notation in subsequent formulae has been modified so as to provide a framework by which one may incorporate blocking variables in later sections.

First, consider the following model for the FFSP setting:

$$
y_{k(j)}=f(\mathrm{WP} \text { effects })+e_{j}+g(\mathrm{SP} \text { effects })+\epsilon_{k(j)},
$$

where $j=1, \ldots, 2^{n_{1}-k_{1}}$ and $k=1, \ldots, 2^{n_{2}-k_{2}}$, with the WP error terms, $e_{j}$, having variance $\sigma_{w}^{2}$, and the SP errors, $e_{k(j)}$, having variance $\sigma_{o}^{2}$. The model term " $y_{k(j)}$ " refers to the response arising from the $k^{\text {th }}$ SP within the $j^{\text {th }}$ WP. The term " $e_{j}$ " refers to the random error arising from the $j^{t h}$ WP unit. Similarly, the term " $\epsilon_{k(j)}$ " refers to the random error arising from the $k^{\text {th }} \mathrm{SP}$ within the $j^{\text {th }}$ WP.

As noted, Bisgaard (2000) only investigated variance forms for FFSP designs. In this setting, Bisgaard (2000) demonstrated that the variance of an estimated WP effect (say, $\widehat{A}$ ) is given by 


$$
\begin{aligned}
\operatorname{Var}(\widehat{A}) & =\operatorname{Var}\left\{\frac{2}{N}\left(2^{n_{2}-k_{2}} \sum_{j=1}^{2^{n_{1}-k_{1}}} \mp e_{j}+\sum_{j=1}^{2^{n_{1}-k_{1}}} \sum_{k=1}^{2^{n_{2}-k_{2}}} \mp \epsilon_{k(j)}\right)\right\} \\
& =\frac{4}{N^{2}}\left(2^{2\left(n_{2}-k_{2}\right)} 2^{n_{1}-k_{1}} \sigma_{w}^{2}+2^{n_{1}-k_{1}} 2^{n_{2}-k_{2}} \sigma_{o}^{2}\right) \\
& =\frac{4}{N}\left(2^{n_{2}-k_{2}} \sigma_{w}^{2}+\sigma_{o}^{2}\right),
\end{aligned}
$$

where $N=2^{\left(n_{1}+n_{2}\right)-\left(k_{1}+k_{2}\right)}$. Bisgaard (2000) also showed that the variance of an estimated SP effect (say, $\hat{p}$ ) is given by

$$
\begin{aligned}
\operatorname{Var}(\hat{p}) & =\operatorname{Var}\left\{\frac{2}{N} \sum_{j=1}^{2^{n_{1}-k_{1}}} \sum_{k=1}^{2^{n_{2}-k_{2}}} \pm \epsilon_{k(j)}\right\} \\
& =\frac{4}{N^{2}} 2^{n_{1}-k_{1}} 2^{n_{2}-k_{2}} \sigma_{o}^{2} \\
& =\frac{4}{N} \sigma_{o}^{2} .
\end{aligned}
$$

Applying the above results to a $2^{(3+3)-(0+1)}$ FFSP design (with $r=A B C p q$ ) we find that

$$
\operatorname{Var}(\widehat{A})=\frac{1}{2} \sigma_{w}^{2}+\frac{1}{8} \sigma_{o}^{2}
$$

and

$$
\operatorname{Var}(\hat{p})=\frac{1}{8} \sigma_{o}^{2}
$$

Note that if a FFSP design is replicated $r$ times, the preceding formulae are easily adapted by letting $N=r 2^{\left(n_{1}+n_{2}\right)-\left(k_{1}+k_{2}\right)}$. (The reader should observe that the use of " $r$ " to denote the number of replicates is not related to $r=A B C p q$ in the preceding paragraph.)

\subsubsection{Variance Forms for BFFSP Designs with Pure WP Blocking}

Recall that pure WP blocking requires that blocking variables be generated exclusively by WP factors. An extension of the model in Equation 4.1 is now provided so 
as to account for the presence of the blocking variables:

$$
y_{k(i j)}=f(\text { WP effects })+e_{j(i)}+g(\text { SP effects })+\epsilon_{k(i j)},
$$

where, $i=1, \ldots, 2^{b_{1}} ; j=1, \ldots, 2^{n_{1}-k_{1}-b_{1}} ; k=1, \ldots, 2^{n_{2}-k_{2}} ; b_{1}$ is the number of pure WP blocking variables; $2^{b_{1}}$ is the number of blocks. The model term " $y_{k(i j)}$ " refers to the response arising from the $k^{\text {th }}$ SP within the $j^{\text {th }}$ WP which, in turn, is within the $i^{\text {th }}$ block. The term " $e_{j(i)}$ " refers to the random error arising from the $j^{\text {th }}$ WP unit within the $i^{\text {th }}$ block. Similarly, the term " $\epsilon_{k(i j)}$ " refers to the random error arising from the $k^{\text {th }} \mathrm{SP}$ within the $j^{\text {th }}$ WP which, in turn, is within the $i^{\text {th }}$ block.

Note the absence of an error term for block effects in Equation 4.2. Many authors discourage the comparison of block means (Anderson and McLean, 1974; Montgomery, 2001) due to concerns regarding the normality of the block error terms and whether or not tests for block effects are meaningful, primarily because all of the randomization takes place within blocks. For these reasons we present a simpler model for the BFFSP design-one without a separate block error term. It can be shown that excluding the block error term will not affect the variance forms of either the WP or SP effects.

The derivation of the effect variances for a BFFSP design with pure WP blocking is best demonstrated by way of example. Table A.2 (in Appendix A) provides the standard run order of the $2^{(3+3)-(0+1) \pm(1+0)}$ BFFSP design having DCS $I=$ $A B C \beta_{1}=A B p q r=C p q r \beta_{1}$, a design first introduced in Chapter 3. In order to aid the development of the effect variances, the error terms corresponding to each treatment combination are also given in Table A.2. Assuming a fixed effects model (where factor levels are specifically pre-selected by the experimenter) we need only focus on the " $e$ 's" and " $\epsilon$ 's" when calculating variances of estimated effects. For example, consider a token WP main effect, say $A$. By adding the error terms in Table A.2, using the signs of $A$, we see that

$$
\begin{aligned}
\widehat{A}= & \frac{1}{16}\left(-4 e_{1(1)}+4 e_{2(1)}+4 e_{3(1)}-4 e_{4(1)}+4 e_{1(2)}-4 e_{2(2)}-4 e_{3(2)}+4 e_{4(2)}\right)+ \\
& \frac{1}{16}\left(-\sum_{k=1}^{4} \epsilon_{k(11)}+\sum_{k=1}^{4} \epsilon_{k(12)}+\sum_{k=1}^{4} \epsilon_{k(13)}-\sum_{k=1}^{4} \epsilon_{k(14)}+\sum_{k=1}^{4} \epsilon_{k(21)}-\sum_{k=1}^{4} \epsilon_{k(22)}-\right. \\
& \left.\sum_{k=1}^{4} \epsilon_{k(23)}+\sum_{k=1}^{4} \epsilon_{k(24)}\right)
\end{aligned}
$$




$$
\begin{aligned}
= & \frac{1}{16}\left(4 e_{2(1)}+4 e_{3(1)}+4 e_{1(2)}+4 e_{4(2)}-4 e_{1(1)}-4 e_{4(1)}-4 e_{2(2)}-4 e_{3(2)}\right)+ \\
& \frac{1}{16}\left(\sum_{k=1}^{4} \epsilon_{k(12)}+\sum_{k=1}^{4} \epsilon_{k(13)}+\sum_{k=1}^{4} \epsilon_{k(21)}+\sum_{k=1}^{4} \epsilon_{k(24)}-\sum_{k=1}^{4} \epsilon_{k(11)}-\sum_{k=1}^{4} \epsilon_{k(14)}-\right. \\
& \left.\sum_{k=1}^{4} \epsilon_{k(22)}-\sum_{k=1}^{4} \epsilon_{k(23)}\right) .
\end{aligned}
$$

This implies that,

$$
\begin{aligned}
\operatorname{Var}(\widehat{A})= & \operatorname{Var}\left\{\frac { 1 } { 1 6 } \left(4 e_{2(1)}+4 e_{3(1)}+4 e_{1(2)}+4 e_{4(2)}-4 e_{1(1)}-4 e_{4(1)}-4 e_{2(2)}-\right.\right. \\
& \left.4 e_{3(2)}\right)+\frac{1}{16}\left(\sum_{k=1}^{4} \epsilon_{k(12)}+\sum_{k=1}^{4} \epsilon_{k(13)}+\sum_{k=1}^{4} \epsilon_{k(21)}+\sum_{k=1}^{4} \epsilon_{k(24)}-\sum_{k=1}^{4} \epsilon_{k(11)}-\right. \\
& \left.\left.\sum_{k=1}^{4} \epsilon_{k(14)}-\sum_{k=1}^{4} \epsilon_{k(22)}-\sum_{k=1}^{4} \epsilon_{k(23)}\right)\right\} \\
= & \frac{8\left(4^{2}\right)}{16^{2}} \sigma_{w}^{2}+\frac{32}{16^{2}} \sigma_{o}^{2} \\
= & \frac{1}{2} \sigma_{w}^{2}+\frac{1}{8} \sigma_{o}^{2} .
\end{aligned}
$$

Similarly, consider a SP main effect, say $p$. Again, by adding the error terms in Table A.2, using the signs of $p$, we see that

$$
\begin{aligned}
\hat{p}= & \frac{1}{16}\left(\sum_{k=2,4} \epsilon_{k(11)}-\sum_{k=1,3} \epsilon_{k(11)}+\sum_{k=2,4} \epsilon_{k(12)}-\sum_{k=1,3} \epsilon_{k(12)}+\sum_{k=2,4} \epsilon_{k(13)}-\sum_{k=1,3} \epsilon_{k(13)}+\right. \\
& \sum_{k=2,4} \epsilon_{k(14)}-\sum_{k=1,3} \epsilon_{k(14)}+\sum_{k=2,4} \epsilon_{k(21)}-\sum_{k=1,3} \epsilon_{k(21)}+\sum_{k=2,4} \epsilon_{k(22)}-\sum_{k=1,3} \epsilon_{k(22)}+ \\
& \left.\sum_{k=2,4} \epsilon_{k(23)}-\sum_{k=1,3} \epsilon_{k(23)}+\sum_{k=2,4} \epsilon_{k(24)}-\sum_{k=1,3} \epsilon_{k(24)}\right) \\
= & \frac{1}{16}\left(\sum_{k=2,4} \epsilon_{k(11)}+\sum_{k=2,4} \epsilon_{k(12)}+\sum_{k=2,4} \epsilon_{k(13)}+\sum_{k=2,4} \epsilon_{k(14)}+\sum_{k=2,4} \epsilon_{k(21)}+\sum_{k=2,4} \epsilon_{k(22)}+\right. \\
& \sum_{k=2,4} \epsilon_{k(23)}+\sum_{k=2,4} \epsilon_{k(24)}-\sum_{k=1,3} \epsilon_{k(11)}-\sum_{k=1,3} \epsilon_{k(12)}-\sum_{k=1,3} \epsilon_{k(13)}-\sum_{k=1,3} \epsilon_{k(14)}- \\
& \sum_{k=1,3} \epsilon_{k(21)}-\sum_{k=1,3} \epsilon_{k(22)}-\sum_{k=1.3} \epsilon_{k(23)}-\sum_{k=1,3} \epsilon_{k(24)} .
\end{aligned}
$$


Note that the WP errors, $e_{j(i)}$, cancel because of an equal number of + 's and -'s within each WP treatment combination. This result implies that,

$$
\begin{aligned}
\operatorname{Var}(\hat{p}) & =\frac{32}{16^{2}} \sigma_{o}^{2} \\
& =\frac{1}{8} \sigma_{o}^{2} .
\end{aligned}
$$

In general, the variance of WP main effects or interactions involving only WP factors (along with all of their aliases) will have the form,

$$
\begin{aligned}
\operatorname{Var}(\widehat{A}) & =\operatorname{Var}\left\{\frac{2}{N}\left(2^{n_{2}-k_{2}} \sum_{i=1}^{2^{b_{1}}} \sum_{j=1}^{2^{n_{1}-k_{1}-b_{1}}} \mp e_{j(i)}+\sum_{i=1}^{2^{b_{1}}} \sum_{j=1}^{2^{n_{1}-k_{1}-b_{1}}} \sum_{k=1}^{2^{n_{2}-k_{2}}} \mp \epsilon_{k(i j)}\right)\right\} \\
& =\frac{4}{N^{2}}\left(2^{2\left(n_{2}-k_{2}\right)} 2^{b_{1}} 2^{n_{1}-k_{1}-b_{1}} \sigma_{w}^{2}+2^{b_{1}} 2^{n_{1}-k_{1}-b_{1}} 2^{n_{2}-k_{2}} \sigma_{o}^{2}\right) \\
& =\frac{4}{N}\left(2^{n_{2}-k_{2}} \sigma_{w}^{2}+\sigma_{o}^{2}\right),
\end{aligned}
$$

with $N=2^{b_{1}} 2^{n_{1}-k_{1}-b_{1}} 2^{n_{2}-k_{2}}=2^{\left(n_{1}+n_{2}\right)-\left(k_{1}+k_{2}\right)}$.

The SP main effects and interactions involving at least one SP factor that are not aliased with either WP main effects or interactions involving only WP factors will have variances of the form

$$
\begin{aligned}
\operatorname{Var}(\hat{p}) & =\operatorname{Var}\left\{\frac{2}{N} \sum_{i=1}^{2^{b_{1}}} \sum_{j=1}^{2^{n_{1}-k_{1}-b_{1}}} \sum_{k=1}^{2^{n_{2}-k_{2}}} \mp \epsilon_{k(i j)}\right\} \\
& =\frac{4}{N^{2}}\left(2^{b_{1}} 2^{n_{1}-k_{1}-b_{1}} 2^{n_{2}-k_{2}} \sigma_{o}^{2}\right) \\
& =\frac{4}{N} \sigma_{o}^{2}
\end{aligned}
$$

where $N=2^{\left(n_{1}+n_{2}\right)-\left(k_{1}+k_{2}\right)}$. (Note that if a FFSP design is replicated $r$ times, the preceding formulae are easily adapted by letting $N=r 2^{\left(n_{1}+n_{2}\right)-\left(k_{1}+k_{2}\right)}$.)

Even though the intermediate steps of the variance derivations differ between FFSP designs and BFFSP designs with pure WP blocking, note that the final form of the variance for WP effects $(\operatorname{Var}(\widehat{A}))$ is identical. Similarly, the variance of SP effects $(\operatorname{Var}(\hat{p}))$ is the same for both FFSP designs and BFFSP designs with pure WP blocking. 


\subsubsection{Variance Forms for BFFSP Designs with Blocking via Separation}

Recall that, under separation, blocking variables (separators) may be generated by exclusively using SP factors or by using SP factors in tandem with WP factors. Under separation, the BFFSP model can be reformulated as follows,

$$
y_{k(i j)}=f(\text { WP effects })+e_{j(i)}+g(\text { SP effects })+\epsilon_{k(i j)}
$$

where, $i=1, \ldots, 2^{b_{2}} ; j=1, \ldots, 2^{n_{1}-k_{1}} ; k=1, \ldots, 2^{n_{2}-k_{2}-b_{2}} ; b_{2}$ is the number of separators; $2^{b_{2}}$ is the number of blocks.

Table A.3 (in Appendix A) lists the standard run order of the $2^{(3+3)-(0+1) \pm(0+1)}$ BFFSP design having DCS $I=A B C p r=A B q \delta_{1}=C p q r \delta_{1}$ (this design was first introduced in Chapter 3). To assist in the development of the effect variances the error terms corresponding to each treatment combination are also provided in Table A.3.

We again assume a fixed effects model so that we need only to focus upon the " $e$ 's" and " $\epsilon$ 's" when calculating variances of the estimated effects. For example, consider a token WP main effect, say $A$. By adding the error terms in Table A.3, using the signs of $A$, we see that

$$
\begin{aligned}
\widehat{A}= & \frac{1}{16}\left(-2 e_{1(1)}+2 e_{2(1)}-\cdots+2 e_{8(1)}-2 e_{1(2)}+2 e_{2(2)}-\cdots+2 e_{8(2)}\right)+ \\
& \frac{1}{16}\left(-\sum_{k=1}^{2} \epsilon_{k(11)}+\sum_{k=1}^{2} \epsilon_{k(12)}-\cdots+\sum_{k=1}^{2} \epsilon_{k(18)}-\sum_{k=1}^{2} \epsilon_{k(21)}+\sum_{k=1}^{2} \epsilon_{k(22)}-\right. \\
& \left.\cdots+\sum_{k=1}^{2} \epsilon_{k(28)}\right) \\
= & \frac{1}{16}\left(2 e_{2(1)}+\cdots+2 e_{8(1)}+2 e_{2(2)}+\cdots+2 e_{8(2)}-2 e_{1(1)}-\cdots-2 e_{7(1)}-2 e_{1(2)}-\right. \\
& \left.\ldots-2 e_{7(2)}\right)+\frac{1}{16}\left(\sum_{k=1}^{2} \epsilon_{k(12)}+\cdots+\sum_{k=1}^{2} \epsilon_{k(18)}+\sum_{k=1}^{2} \epsilon_{k(22)}+\cdots+\sum_{k=1}^{2} \epsilon_{k(28)}-\right. \\
& \left.\sum_{k=1}^{2} \epsilon_{k(11)}-\cdots-\sum_{k=1}^{2} \epsilon_{k(17)}-\sum_{k=1}^{2} \epsilon_{k(21)}-\cdots-\sum_{k=1}^{2} \epsilon_{k(27)}\right) .
\end{aligned}
$$


Therefore,

$$
\begin{aligned}
\operatorname{Var}(\widehat{A}) & =\frac{16\left(2^{2}\right)}{16^{2}} \sigma_{w}^{2}+\frac{32}{16^{2}} \sigma_{o}^{2} \\
& =\frac{1}{4} \sigma_{w}^{2}+\frac{1}{8} \sigma_{o}^{2} .
\end{aligned}
$$

Note that under separation the coefficient of $\sigma_{w}^{2}$ has decreased from $\frac{1}{2}$ to $\frac{1}{4}$. (Compare with the FFSP and pure WP blocking design scenarios.) The preceding result demonstrates one of the consequences of separation, namely pseudo-replication, first discussed in Chapter 3. Specifically, we recall that each separator results in a doubling of the number of distinct runs at the WP level, causing increased precision for estimates of WP effects. (Conversely, recall that each additional separator causes a decrease, by a factor of two, in the number of SP treatment combinations associated with each WP treatment combination, when compared to the pure WP blocking approach.) This doubling of distinct WP runs then results in a decrease, by a factor of two, in the coefficient of $\sigma_{w}^{2}$. Consequently, increased precision is achieved for testing the statistical significance of WP effects.

Now consider a SP effect, say $p$. By adding the error terms in Table A.3, using the signs of $p$, we see that

$$
\begin{aligned}
\hat{p}= & \frac{1}{16}\left(-\epsilon_{1(11)}+\epsilon_{2(11)}-\cdots-\epsilon_{1(18)}+\epsilon_{2(18)}-\epsilon_{1(21)}+\epsilon_{2(21)}-\cdots-\epsilon_{1(28)}+\right. \\
& \left.\epsilon_{2(28)}\right) \\
= & \frac{1}{16} \sum_{i=1}^{2} \sum_{j=1}^{8} \sum_{k=1}^{2} \mp \epsilon_{k(i j)} .
\end{aligned}
$$

Note that the WP errors, $e_{j(i)}$, cancel because of an equal number of + 's and -'s within each WP treatment combination. This implies that

$$
\begin{aligned}
\operatorname{Var}(\hat{p}) & =\frac{32}{16^{2}} \sigma_{o}^{2} \\
& =\frac{1}{8} \sigma_{o}^{2} .
\end{aligned}
$$

For BFFSP designs with blocking via separation, the variance of WP main effects 
or interactions involving only WP factors (along with their aliases) will be of the form,

$$
\begin{aligned}
\operatorname{Var}(\widehat{A}) & =\operatorname{Var}\left\{\frac{2}{N}\left(2^{n_{2}-k_{2}-b_{2}} \sum_{i=1}^{2^{b_{2}}} \sum_{j=1}^{2^{n_{1}-k_{1}}} \mp e_{j(i)}+\sum_{i=1}^{2^{b_{2}}} \sum_{j=1}^{2^{n_{1}-k_{1}}} \sum_{k=1}^{n_{2}-k_{2}-b_{2}} \mp \epsilon_{k(i j)}\right)\right\} \\
& =\frac{4}{N^{2}}\left(2^{2\left(n_{2}-k_{2}-b_{2}\right)} 2^{b_{2}} 2^{n_{1}-k_{1}} \sigma_{w}^{2}+2^{b_{2}} 2^{n_{1}-k_{1}} 2^{n_{2}-k_{2}-b_{2}} \sigma_{o}^{2}\right) \\
& =\frac{4}{N}\left(2^{n_{2}-k_{2}-b_{2}} \sigma_{w}^{2}+\sigma_{o}^{2}\right),
\end{aligned}
$$

with $N=2^{b_{2}} 2^{n_{2}-k_{2}-b_{2}} 2^{n_{1}-k_{1}}=2^{\left(n_{1}+n_{2}\right)-\left(k_{1}+k_{2}\right)}$. By examining the final form of $\operatorname{Var}(\widehat{A})$, we see that the coefficient of $\sigma_{w}^{2}$ will decrease by a factor of two for each additional separator. However, as discussed in Chapter 3 the allowable number of separators is given by the inequality, $1 \leq b_{2} \leq n_{2}-k_{2}-1$.

SP main effects and interactions involving at least one SP factor that are not aliased with either WP main effects or interactions involving only WP factors will have variances given by

$$
\begin{aligned}
\operatorname{Var}(\hat{p}) & =\operatorname{Var}\left\{\frac{2}{N} \sum_{i=1}^{2^{b_{2}}} \sum_{j=1}^{2^{n_{1}-k_{1}}} \sum_{k=1}^{2^{n_{2}-k_{2}-b_{2}}} \mp \epsilon_{k(i j)}\right\} \\
& =\frac{4}{N^{2}}\left(2^{b_{2}} 2^{n_{1}-k_{1}} 2^{n_{2}-k_{2}-b_{2}} \sigma_{o}^{2}\right) \\
& =\frac{4}{N} \sigma_{o}^{2}
\end{aligned}
$$

where $N=2^{\left(n_{1}+n_{2}\right)-\left(k_{1}+k_{2}\right)}$.

\subsubsection{Variance Forms for BFFSP Designs with Mixed Block- ing}

As we know from Chapter 3, mixed blocking is a natural extension of pure WP blocking and separation in that we simultaneously use pure WP blocking variables and separators in this blocking approach. Under mixed blocking the BFFSP model is given by,

$$
y_{k(i j)}=f(\text { WP effects })+e_{j(i)}+g(\text { SP effects })+\epsilon_{k(i j)},
$$


where, $i=1, \ldots, 2^{b_{1}+b_{2}} ; j=1, \ldots, 2^{n_{1}-k_{1}-b_{1}} ; k=1, \ldots, 2^{n_{2}-k_{2}-b_{2}} ; b_{1}$ is the number of pure WP blocking variables; $b_{2}$ is the number of separators; $2^{b_{1}+b_{2}}$ is the total number of blocks.

Table A.4 (in Appendix A) gives the standard run order of the $2^{(3+3)-(0+1) \pm(1+1)}$ BFFSP design having DCS, $I=A B q r=A B C \beta_{1}=A C p r \delta_{1}=C q r \beta_{1}=B C p q \delta_{1}=$ $B p r \beta_{1} \delta_{1}=A p q \beta_{1} \delta_{1}$ (this design was first introduced in Chapter 3).

Before providing the general variance forms for the mixed blocking approach, we illustrate the variance derivations for a specific WP and SP effect using the $2^{(3+3)-(0+1) \pm(1+1)}$ design.

Assuming a fixed effects model, consider the WP main effect $A$. By adding the error terms in Table A.4, using the signs of $A$, we see that,

$$
\begin{aligned}
\widehat{A}= & \frac{1}{16}\left(-2 e_{1(1)}+2 e_{2(1)}+2 e_{3(1)}-2 e_{4(1)}+2 e_{1(2)}-2 e_{2(2)}-2 e_{3(2)}+2 e_{4(2)}-\right. \\
& \left.2 e_{1(3)}+2 e_{2(3)}+2 e_{3(3)}-2 e_{4(3)}+2 e_{1(4)}-2 e_{2(4)}-2 e_{3(4)}+2 e_{4(4)}\right)+ \\
& \frac{1}{16}\left(-\sum_{k=1}^{2} \epsilon_{k(11)}+\sum_{k=1}^{2} \epsilon_{k(12)}+\sum_{k=1}^{2} \epsilon_{k(13)}-\sum_{k=1}^{2} \epsilon_{k(14)}+\sum_{k=1}^{2} \epsilon_{k(21)}-\sum_{k=1}^{2} \epsilon_{k(22)}-\right. \\
& \sum_{k=1}^{2} \epsilon_{k(23)}+\sum_{k=1}^{2} \epsilon_{k(24)}-\sum_{k=1}^{2} \epsilon_{k(31)}+\sum_{k=1}^{2} \epsilon_{k(32)}+\sum_{k=1}^{2} \epsilon_{k(33)}-\sum_{k=1}^{2} \epsilon_{k(34)}+ \\
& \left.\sum_{k=1}^{2} \epsilon_{k(41)}-\sum_{k=1}^{2} \epsilon_{k(42)}-\sum_{k=1}^{2} \epsilon_{k(43)}+\sum_{k=1}^{2} \epsilon_{k(44)}\right) \\
= & \frac{1}{16}\left(2 e_{2(1)}+2 e_{3(1)}+2 e_{1(2)}+2 e_{4(2)}+2 e_{2(3)}+2 e_{3(3)}+2 e_{1(4)}+2 e_{4(4)}-\right. \\
& \left.2 e_{1(1)}-2 e_{4(1)}-2 e_{2(2)}-2 e_{3(2)}-2 e_{1(3)}-2 e_{4(3)}-2 e_{2(4)}-2 e_{3(4)}\right)+ \\
& \frac{1}{16}\left(\sum_{k=1}^{2} \epsilon_{k(12)}+\sum_{k=1}^{2} \epsilon_{k(13)}+\sum_{k=1}^{2} \epsilon_{k(21)}+\sum_{k=1}^{2} \epsilon_{k(24)}+\sum_{k=1}^{2} \epsilon_{k(32)}+\sum_{k=1}^{2} \epsilon_{k(33)}+\right.
\end{aligned}
$$




$$
\begin{aligned}
& \sum_{k=1}^{2} \epsilon_{k(41)}+\sum_{k=1}^{2} \epsilon_{k(44)}-\sum_{k=1}^{2} \epsilon_{k(11)}-\sum_{k=1}^{2} \epsilon_{k(14)}-\sum_{k=1}^{2} \epsilon_{k(22)}-\sum_{k=1}^{2} \epsilon_{k(23)}- \\
& \left.\sum_{k=1}^{2} \epsilon_{k(31)}-\sum_{k=1}^{2} \epsilon_{k(34)}-\sum_{k=1}^{2} \epsilon_{k(42)}-\sum_{k=1}^{2} \epsilon_{k(43)}\right) .
\end{aligned}
$$

Therefore,

$$
\begin{aligned}
\operatorname{Var}(\widehat{A}) & =\frac{16\left(2^{2}\right)}{16^{2}} \sigma_{w}^{2}+\frac{32}{16^{2}} \sigma_{o}^{2} \\
& =\frac{1}{4} \sigma_{w}^{2}+\frac{1}{8} \sigma_{o}^{2} .
\end{aligned}
$$

From the final form of $\operatorname{Var}(\widehat{A})$ we see that the attributes of separation carry directly over to the mixed blocking scenario. By this we mean that as a consequence of the separator we again obtain increased replication of the WP treatment combinations at the expense of further fractionation at the SP level. This pseudo-replication will provide the experimenter with increased precision in testing for significance of WP effects.

Now consider a SP main effect, say $q$. Again, by adding the error terms in Table A.4, using the signs of $q$, we see that

$$
\begin{aligned}
\hat{q} & =\frac{1}{16}\left(-\epsilon_{1(11)}+\epsilon_{2(11)}-\epsilon_{1(12)}+\epsilon_{2(12)}-\cdots-\epsilon_{1(43)}+\epsilon_{2(43)}-\epsilon_{1(44)}+\epsilon_{2(44)}\right) \\
& =\frac{1}{16} \sum_{i=1}^{4} \sum_{j=1}^{4} \sum_{k=1}^{2} \mp \epsilon_{k(i j)} .
\end{aligned}
$$

Therefore,

$$
\begin{aligned}
\operatorname{Var}(\hat{q}) & =\frac{32}{16^{2}} \sigma_{o}^{2} \\
& =\frac{1}{8} \sigma_{o}^{2} .
\end{aligned}
$$

The general form of the variance for a WP effect is given by, 


$$
\begin{aligned}
\operatorname{Var}(\widehat{A})= & \operatorname{Var}\left\{\frac { 2 } { N } \left(2^{n_{2}-k_{2}-b_{2}} \sum_{i=1}^{2^{b_{1}+b_{2}}} \sum_{j=1}^{2^{n_{1}-k_{1}-b_{1}}} \mp e_{j(i)}+\right.\right. \\
& \left.\left.\sum_{i=1}^{2^{b_{1}+b_{2}}} \sum_{j=1}^{2^{n_{1}-k_{1}-b_{1}}} \sum_{2^{n_{2}-k_{2}-b_{2}}} \mp \epsilon_{k(i j)}\right)\right\} \\
= & \frac{4}{N^{2}}\left\{2^{2\left(n_{2}-k_{2}-b_{2}\right)} 2^{b_{1}+b_{2}} 2^{n_{1}-k_{1}-b_{1}} \sigma_{w}^{2}+2^{b_{1}+b_{2}} 2^{n_{1}-k_{1}-b_{1}} 2^{n_{2}-k_{2}-b_{2}} \sigma_{o}^{2}\right\} \\
= & \frac{4}{N}\left(2^{n_{2}-k_{2}-b_{2}} \sigma_{w}^{2}+\sigma_{o}^{2}\right),
\end{aligned}
$$

with $N=2^{n_{2}-k_{2}-b_{2}} 2^{n_{1}-k_{1}-b_{1}} 2^{b_{1}+b_{2}}=2^{\left(n_{1}+n_{2}\right)-\left(k_{1}+k_{2}\right)}$. The SP main effects and interactions involving at least one SP factor that are not aliased with either WP main effects or interactions involving only WP factors will have variances of the form

$$
\begin{aligned}
\operatorname{Var}(\hat{q}) & =\operatorname{Var}\left\{\frac{2}{N} \sum_{i=1}^{2^{b_{1}+b_{2}}} \sum_{j=1}^{2^{n_{1}-k_{1}-b_{1}}} \sum_{k=1}^{2^{n_{2}-k_{2}-b_{2}}} \mp \epsilon_{k(i j)}\right\} \\
& =\frac{4}{N^{2}}\left\{2^{b_{1}+b_{2}} 2^{n_{1}-k_{1}-b_{1}} 2^{n_{2}-k_{2}-b_{2}} \sigma_{o}^{2}\right\} \\
& =\frac{4}{N} \sigma_{o}^{2}
\end{aligned}
$$

where $N=2^{\left(n_{1}+n_{2}\right)-\left(k_{1}+k_{2}\right)}$.

\subsection{Summary of Results}

In this chapter we reviewed the work of Bisgaard (2000) for the construction of variances for WP and SP effects in FFSP designs. Following this methodology we derived the variances for WP and SP effects in BFFSP designs. Specifically we considered the three scenarios:

1. BFFSP designs with pure WP blocking.

2. BFFSP designs with blocking via separation. 
3. BFFSP designs with mixed blocking.

Table 4.1 summarizes the results and makes it readily apparent that when compared to pure WP blocking, both separation and mixed blocking provide greater precision for testing at the WP level.

Table 4.1: Variance Forms of WP and SP Effects in FFSP and BFFSP Designs.

\begin{tabular}{||lcc||}
\hline Design & WP Effects & SP Effects \\
\hline FFSP and Pure WP Blocking & $\frac{4}{N}\left(2^{n_{2}-k_{2}} \sigma_{w}^{2}+\sigma_{o}^{2}\right)$ & $\frac{4}{N} \sigma_{o}^{2}$ \\
Separation & $\frac{4}{N}\left(2^{n_{2}-k_{2}-b_{2}} \sigma_{w}^{2}+\sigma_{o}^{2}\right)$ & $\frac{4}{N} \sigma_{o}^{2}$ \\
Mixed Blocking & $\frac{4}{N}\left(2^{n_{2}-k_{2}-b_{2}} \sigma_{w}^{2}+\sigma_{o}^{2}\right)$ & $\frac{4}{N} \sigma_{o}^{2}$ \\
\hline
\end{tabular}

One may use the following rules in order to correctly select the appropriate error term (WP or SP) for assessing the statistical significance of BFFSP design contrasts. For effects not confounded with blocks the rules are as follows:

1. WP main effects and interactions involving only WP factors are compared to the WP error.

2. SP main effects and interactions involving at least one SP factor that are aliased with either WP main effects or interactions involving only WP factors are compared to the WP error.

3. SP main effects and interactions involving at least one SP factor that are not aliased with either WP main effects or interactions involving only WP factors are compared to the SP error.

These rules are those originally developed for the FFSP setting by Bisgaard (2000), and subsequently summarized by Bingham and Sitter (2001). 


\section{Chapter 5}

\section{MA BFFSP Design Search}

Until now there has been no formal attempt to construct catalogues of BFFSP designs incorporating any one of the three blocking schemes discussed in Chapter 3 . This has prohibited a rank ordering of BFFSP designs using predefined optimality criteria. This chapter will detail methods by which one may "search" for and obtain two-level BFFSP designs ranked according to the MA criterion. We shall also see that for a given value of $n_{1}, n_{2} ; k_{1}, k_{2} ; b_{1}, b_{2}$ there may be many $2^{\left(n_{1}+n_{2}\right)-\left(k_{1}+k_{2}\right) \pm\left(b_{1}+b_{2}\right)}$ DCSs possessing MA. To further differentiate between the "goodness" of these MA designs additional optimality criteria will be presented for ranking purposes.

\subsection{An Introduction to Search Algorithms and Re- lated Issues}

In recent years, for the purpose of constructing catalogues of optimal $\mathrm{FF}, \mathrm{BFF}$ and FFSP designs several algorithms have been introduced to assist in searching through all design possibilities.

Franklin and Bailey (1977) introduced the search-table approach for identifying FF designs with particular estimation properties. Chen, Sun and Wu (1993) introduced a sequential approach for constructing complete sets of two-level and three-level FF designs ranked according to the MA criterion. Huang et al. (1998) presented an ad-hoc method for finding MA FFSP designs while Bingham and Sitter (1999a) combined the existing search-table and sequential approaches to obtain a more efficient search algorithm for constructing MA FFSP designs. 
The objective of this chapter is not to develop the most efficient algorithm (computationally speaking) for searching for MA BFFSP designs. Rather, the aim is to provide an algorithm by adapting portions of existing algorithms, used previously in the search for "optimal" FF, BFF and FFSP designs, to the BFFSP setting.

\subsubsection{Search-Table Approach for FFSP Designs}

Bingham and Sitter (1999a) provide an example that demonstrates an adaptation of the search-table technique in the search for FFSP designs. We now summarize this example using appropriate notational changes.

The search-table is a two-way table with $2^{\left(n_{1}+n_{2}\right)-\left(k_{1}+k_{2}\right)}-\left(n_{1}-k_{1}\right)-\left(n_{2}-k_{2}\right)-1$ rows and $k_{1}+k_{2}$ columns. The columns are headed by the $k_{1}+k_{2}$ WP and SP added factors of the $2^{\left(n_{1}+n_{2}\right)-\left(k_{1}+k_{2}\right)}$ FFSP design. The column headers are arranged so that the WP added factors appear before the SP added factors. The products of the basic factors serve as the row headers. The rows are sorted by level of the design (WP followed by SP) then by word length. This enables the WP basic interactions to appear before the products containing both basic WP and SP factors. Finally, the elements of the table are the products of the row and column headers.

Example 5.1.1 For a $2^{(5+4)-(2+3)}$ FFSP design with WP factors $A, B, C, D$ and $E$ and SP factors $p, q, r$ and $s$, the search table is given in Table 5.1.

To preserve the split-plot nature of the design we know that WP generators cannot contain any SP factors and SP factors cannot be assigned to interactions consisting of only WP factors; consequently, these prohibited generators need not be considered in the search process. Following Bingham and Sitter (1999a) we use -'s in the search-table to demonstrate the exclusion of prohibited generators.

To construct a $2^{(5+4)-(2+3)}$ design, one must take a single generator from each of the five columns of Table 5.1. The first possible design has generators $g_{1}=A B D, g_{2}=$ $A B E, g_{3}=A p q, g_{4}=A p r$ and $g_{5}=A p s$. One may then form the DCS for the design by calculating all $2^{5}-1$ possible products of the five generators. Subsequently, the resolution and WLP of the design may be obtained from the DCS.

The second FFSP design considered via the search-table approach has $g_{5}=B p s$, while $g_{1}$ through $g_{4}$ remain the same. Thus the search algorithm proceeds from left to right across columns and from top to bottom within a column. 
Table 5.1: The Search-Table for the $2^{(5+4)-(2+3)}$ Design in Example 5.1.1.

\begin{tabular}{l||ccccc}
\hline \hline & $\mathrm{D}$ & $\mathrm{E}$ & $\mathrm{q}$ & $\mathrm{r}$ & $\mathrm{s}$ \\
\hline $\mathrm{AB}$ & $\mathrm{ABD}$ & $\mathrm{ABE}$ & - & - & - \\
$\mathrm{AC}$ & $\mathrm{ACD}$ & $\mathrm{ACE}$ & - & - & - \\
$\mathrm{BC}$ & $\mathrm{BCD}$ & $\mathrm{BCE}$ & - & - & - \\
$\mathrm{ABC}$ & $\mathrm{ABCD}$ & $\mathrm{ABCE}$ & - & - & - \\
$\mathrm{Ap}$ & - & - & $\mathrm{Apq}$ & $\mathrm{Apr}$ & $\mathrm{Aps}$ \\
$\mathrm{Bp}$ & - & - & $\mathrm{Bpq}$ & $\mathrm{Bpr}$ & $\mathrm{Bps}$ \\
$\mathrm{Cp}$ & - & - & $\mathrm{Cpq}$ & $\mathrm{Cpr}$ & $\mathrm{Cps}$ \\
$\mathrm{ABp}$ & - & - & $\mathrm{ABpq}$ & $\mathrm{ABpr}$ & $\mathrm{ABps}$ \\
$\mathrm{ACp}$ & - & - & $\mathrm{ACpq}$ & $\mathrm{ACpr}$ & $\mathrm{ACps}$ \\
$\mathrm{BCp}$ & - & - & $\mathrm{BCpq}$ & $\mathrm{BCpr}$ & $\mathrm{BCps}$ \\
$\mathrm{ABCp}$ & - & - & $\mathrm{ABCpq}$ & $\mathrm{ABCpr}$ & $\mathrm{ABCps}$ \\
\hline
\end{tabular}

The search-table requires that $N_{1}=\left(2^{n_{1}-k_{1}}-\left(n_{1}-k_{1}\right)-1\right)^{k_{1}}$ WP designs and $N_{2}=\left(2^{\left(n_{1}+n_{2}\right)-\left(k_{1}+k_{2}\right)}-\left(\left(n_{1}+n_{2}\right)-\left(k_{1}+k_{2}\right)\right)-\left(2^{n_{1}-k_{1}}-\left(n_{1}-k_{1}\right)-1\right)-1\right)^{k_{2}} \mathrm{SP}$ designs be searched. Hence, $N_{1} \times N_{2}$ designs must be considered when constructing all possible FFSP designs using the search-table technique.

\subsubsection{Design Isomorphism and Practical Considerations}

Two FF designs $D_{1}$ and $D_{2}$ are said to be isomorphic if we can obtain $D_{2}$ from $D_{1}$ by relabeling the factors of $D_{1}$. For example, consider the simple case of two $2^{8-2}$ FF designs. Suppose that $D_{1}$ has generators $g_{1}=A B C F$ and $g_{2}=C D E$, while $D_{2}$ has generators $g_{1}=A B C E$ and $g_{2}=C D F$. These two designs are isomorphic since $D_{2}$ can be obtained from $D_{1}$ by relabeling $\mathrm{F}$ as $\mathrm{E}$ and $\mathrm{E}$ as $\mathrm{F}$. It has been noted (Chen et al., 1993) that isomorphic designs are essentially equivalent (for example, two isomorphic designs will possess the same WLP). In the context of FFSP designs, the WP or SP designations of factors must also be preserved for isomorphic designs.

The goal of Bingham and Sitter (1999a) was to obtain catalogs of nonisomorphic $2^{\left(n_{1}+n_{2}\right)-\left(k_{1}+k_{2}\right)}$ FFSP designs ranked according to the MA criterion. To do this, 
Bingham and Sitter (1999a) prescribed an isomorphism test for the split-plot setting that entailed several computationally intensive steps. Bingham and Sitter (1999a) noted that it was inefficient to search for nonisomorphic designs via the search-table because of the large number of designs that the search-table required one to assess. As a reasonable solution to this problem, Bingham and Sitter (1999a) proceeded to develop a more efficient algorithm known as the combined approach.

Our intent is not to distinguish between nonismorphic BFFSP designs. Rather, we will focus specifically upon the search for MA BFFSP designs without much regard to their isomorphism status. Therefore, the search-table approach (despite its computational deficiencies) will suffice for our purposes.

\subsection{The Search-Table Approach for BFFSP De- signs}

The three approaches to blocking FFSP designs will necessitate three variations of the search-table. We will now consider each variation in turn.

\subsubsection{The Search-Table for Pure WP Blocking}

Recall that pure WP blocking implies that blocking variables must be generated solely by WP factors. With this in mind, we shall see that the dimensions of the search-table are altered slightly when compared with the FFSP setting.

The number of rows in the search-table is $2^{\left(n_{1}+n_{2}\right)-\left(k_{1}+k_{2}\right)}-\left(n_{1}-k_{1}\right)-\left(n_{2}-k_{2}\right)-$ 1 , the same number as in the unblocked setting. However, the number of column headers now increases by $b_{1}$, the number of pure WP blocking variables, to $k_{1}+k_{2}+$ $b_{1}$. Therefore, the search-table now requires that $N_{1}=\left(2^{n_{1}-k_{1}}-\left(n_{1}-k_{1}\right)-1\right)^{k_{1}+b_{1}}$ WP designs be searched. The number of SP designs to be considered, $N_{2}$, remains unchanged from Section 5.1.1.

Example 5.2.1 Consider $a 2^{(4+2)-(1+1) \pm(1+0)}$ BFFSP design. Since the blocking variable, $\beta_{1}$, is generated exclusively by WP factors it appears as a column header before the SP added factor, q; see Table 5.2. Note that it would make no difference to the search procedure if one was to shift the column headed by $\beta_{1}$ to be in front of the column headed by the WP added factor, D. 
Table 5.2: The Search-Table for the $2^{(4+2)-(1+1) \pm(1+0)}$ Design in Example 5.2.1.

\begin{tabular}{l||ccc}
\hline \hline & $\mathrm{D}$ & $\beta_{1}$ & $\mathrm{q}$ \\
\hline $\mathrm{AB}$ & $\mathrm{ABD}$ & $\mathrm{AB} \beta_{1}$ & - \\
$\mathrm{AC}$ & $\mathrm{ACD}$ & $\mathrm{AC} \beta_{1}$ & - \\
$\mathrm{BC}$ & $\mathrm{BCD}$ & $\mathrm{BC} \beta_{1}$ & - \\
$\mathrm{ABC}$ & $\mathrm{ABCD}$ & $\mathrm{ABC} \beta_{1}$ & - \\
$\mathrm{Ap}$ & - & - & $\mathrm{Apq}$ \\
$\mathrm{Bp}$ & - & - & $\mathrm{Bpq}$ \\
$\mathrm{Cp}$ & - & - & $\mathrm{Cpq}$ \\
$\mathrm{ABp}$ & - & - & $\mathrm{ABpq}$ \\
$\mathrm{ACp}$ & - & - & $\mathrm{ACpq}$ \\
$\mathrm{BCp}$ & - & - & $\mathrm{BCpq}$ \\
$\mathrm{ABCp}$ & - & - & $\mathrm{ABCpq}$ \\
\hline
\end{tabular}

The first BFFSP design for which the DCS and WLP is calculated is the design possessing generators $g_{1}=A B D, g_{2}=A B \beta_{1}$ and $g_{3}=A p q$. The second design encountered has generators, $g_{1}=A B D, g_{2}=A B \beta_{1}$ and $g_{3}=B p q$. Thus, as when searching through all possible FFSP designs, the algorithm proceeds from left to right across columns and from top to bottom within a column. The WLP of the design being currently assessed is then compared with that of the previous design(s). If the current design has less aberration than previous designs, the previous designs can be discarded. Designs possessing the current MA WLP are retained. The algorithm continues until all $N_{1} \times N_{2}$ designs have been searched.

\subsubsection{The Search-Table for Separation}

Recall that blocking via separation allows one to generate blocking variables by exclusively using SP factors or by using SP factors in tandem with WP factors in the blocking generators.

As with pure WP blocking, we see changes in the dimensions of the search-table due to the presence of the separators. Again, the number of rows is $2^{\left(n_{1}+n_{2}\right)-\left(k_{1}+k_{2}\right)}-$ 
$\left(n_{1}-k_{1}\right)-\left(n_{2}-k_{2}\right)-1$; however, the number of column headers becomes $k_{1}+$ $k_{2}+b_{2}$, where $b_{2}$ denotes the number of separators. The search-table requires $N_{1}=$ $\left(2^{n_{1}-k_{1}}-\left(n_{1}-k_{1}\right)-1\right)^{k_{1}}$ and $N_{2}=\left(2^{\left(n_{1}+n_{2}\right)-\left(k_{1}+k_{2}\right)}-\left(\left(n_{1}+n_{2}\right)-\left(k_{1}+k_{2}\right)\right)-\right.$ $\left.\left(2^{n_{1}-k_{1}}-\left(n_{1}-k_{1}\right)-1\right)-1\right)^{k_{2}+b_{2}}$ WP and SP designs to be sorted through, respectively.

Example 5.2.2 Consider a $2^{(4+3)-(1+1) \pm(0+1)}$ BFFSP design. The lone blocking variable, denoted by $\delta_{1}$, appears as a column header after the column headed by the SP added factor, $r$, in Table 5.3. (Note that it would make no difference to the search procedure if one were to switch the ordering of these two columns.) Since the generator for the separator must contain at least one SP factor, - -'s in the table indicate unusable generator candidates.

The algorithm proceeds in a manner similar to that for pure WP blocking. That is, when sorting through the designs, one proceeds from left to right across the columns and from top to bottom within a column, while choosing a single generator from each column. As before, DCSs are formed and WLPs for each design can be compared with one another in the search for the separated design(s) having $M A$.

\subsubsection{The Search-Table for Mixed Blocking}

Recall that mixed blocking entails the use of both pure WP blocking variables and separators.

It is for this third blocking approach in which we observe the most significant changes in the dimensions of the search-table (when compared to the FFSP setting). Although the number of rows remains unchanged, the number of columns is now $k_{1}+k_{2}+b_{1}+b_{2}$. This implies that the number of WP and SP designs to be sorted thru are now $N_{1}=\left(2^{n_{1}-k_{1}}-\left(n_{1}-k_{1}\right)-1\right)^{k_{1}+b_{1}}$ and $N_{2}=\left(2^{\left(n_{1}+n_{2}\right)-\left(k_{1}+k_{2}\right)}-\left(\left(n_{1}+n_{2}\right)-\right.\right.$ $\left.\left.\left(k_{1}+k_{2}\right)\right)-\left(2^{n_{1}-k_{1}}-\left(n_{1}-k_{1}\right)-1\right)-1\right)^{k_{2}+b_{2}}$, respectively. Therefore, $N_{1} \times N_{2}$ splitplot designs possessing mixed blocking must be individually examined. In comparison with $N_{1}$ and $N_{2}$ from the pure WP blocking and separation approaches, it can be seen that the mixed blocking procedure will often require the largest number of designs to sort through in the search-table. 
Table 5.3: The Search-Table for the $2^{(4+3)-(1+1) \pm(0+1)}$ Design in Example 5.2.2.

\begin{tabular}{l||ccc}
\hline \hline & $\mathrm{D}$ & $\mathrm{r}$ & $\delta_{1}$ \\
\hline $\mathrm{AB}$ & $\mathrm{ABD}$ & - & - \\
$\mathrm{AC}$ & $\mathrm{ACD}$ & - & - \\
$\mathrm{BC}$ & $\mathrm{BCD}$ & - & - \\
$\mathrm{ABC}$ & $\mathrm{ABCD}$ & - & - \\
$\mathrm{Ap}$ & - & $\mathrm{Apr}$ & $\mathrm{Ap} \delta_{1}$ \\
$\mathrm{Bp}$ & - & $\mathrm{Bpr}$ & $\mathrm{Bp} \delta_{1}$ \\
$\mathrm{Cp}$ & - & $\mathrm{Cpr}$ & $\mathrm{Cp} \delta_{1}$ \\
$\mathrm{Aq}$ & - & $\mathrm{Aqr}$ & $\mathrm{Aq} \delta_{1}$ \\
$\mathrm{~Bq}$ & - & $\mathrm{Bqr}$ & $\mathrm{Bq} \delta_{1}$ \\
$\mathrm{Cq}$ & - & $\mathrm{Cqr}$ & $\mathrm{Cq} \delta_{1}$ \\
$\mathrm{pq}$ & - & $\mathrm{pqr}$ & $\mathrm{pq} \delta_{1}$ \\
$\mathrm{ABp}$ & - & $\mathrm{ABpr}$ & $\mathrm{ABp} \delta_{1}$ \\
$\mathrm{ACp}$ & - & $\mathrm{ACpr}$ & $\mathrm{ACp} \delta_{1}$ \\
$\mathrm{BCp}$ & - & $\mathrm{BCpr}$ & $\mathrm{BCp} \delta_{1}$ \\
$\mathrm{ABq}$ & - & $\mathrm{ABqr}$ & $\mathrm{ABq} \delta_{1}$ \\
$\mathrm{ACq}$ & - & $\mathrm{ACqr}$ & $\mathrm{ACq} \delta_{1}$ \\
$\mathrm{BCq}$ & - & $\mathrm{BCqr}$ & $\mathrm{BCq} \delta_{1}$ \\
$\mathrm{Apq}$ & - & $\mathrm{Apqr}$ & $\mathrm{Apq} \delta_{1}$ \\
$\mathrm{Bpq}$ & - & $\mathrm{Bpqr}$ & $\mathrm{Bpq} \delta_{1}$ \\
$\mathrm{Cpq}$ & - & $\mathrm{Cpqr}$ & $\mathrm{Cpq} \delta_{1}$ \\
$\mathrm{ABCp}$ & - & $\mathrm{ABCpr}$ & $\mathrm{ABCp} \delta_{1}$ \\
$\mathrm{ABCq}$ & - & $\mathrm{ABCqr}$ & $\mathrm{ABCq} \delta_{1}$ \\
$\mathrm{ABpq}$ & - & $\mathrm{ABpqr}$ & $\mathrm{ABpq} \delta_{1}$ \\
$\mathrm{ACpq}$ & - & $\mathrm{ACpqr}$ & $\mathrm{ACpq} \delta_{1}$ \\
$\mathrm{BCpq}$ & - & $\mathrm{BCpqr}$ & $\mathrm{BCpq} \delta_{1}$ \\
$\mathrm{ABCpq}$ & - & $\mathrm{ABCpqr}$ & $\mathrm{ABCpq} \delta_{1}$ \\
\hline & & &
\end{tabular}

Example 5.2.3 Consider a 32-run $2^{(4+3)-(1+1) \pm(1+1)}$ BFFSP design. The column headings of the search-table, Table 5.4, consist of the WP and SP added factors as 
well as a pure WP blocking variable and a single separator.

Table 5.4: The Search-Table for the $2^{(4+3)-(1+1) \pm(1+1)}$ Design in Example 5.2.3.

\begin{tabular}{l||cccc}
\hline \hline & $\mathrm{D}$ & $\beta_{1}$ & $\mathrm{r}$ & $\delta_{1}$ \\
\hline $\mathrm{AB}$ & $\mathrm{ABD}$ & $\mathrm{AB} \beta_{1}$ & - & - \\
$\mathrm{AC}$ & $\mathrm{ACD}$ & $\mathrm{AC} \beta_{1}$ & - & - \\
$\mathrm{BC}$ & $\mathrm{BCD}$ & $\mathrm{BC} \beta_{1}$ & - & - \\
$\mathrm{ABC}$ & $\mathrm{ABCD}$ & $\mathrm{ABC} \beta_{1}$ & - & - \\
$\mathrm{Ap}$ & - & - & $\mathrm{Apr}$ & $\mathrm{Ap} \delta_{1}$ \\
$\mathrm{Bp}$ & - & - & $\mathrm{Bpr}$ & $\mathrm{Bp} \delta_{1}$ \\
$\mathrm{Cp}$ & - & - & $\mathrm{Cpr}$ & $\mathrm{Cp} \delta_{1}$ \\
$\mathrm{Aq}$ & - & - & $\mathrm{Aqr}$ & $\mathrm{Aq} \delta_{1}$ \\
$\mathrm{~Bq}$ & - & - & $\mathrm{Bqr}$ & $\mathrm{Bq} \delta_{1}$ \\
$\mathrm{Cq}$ & - & - & $\mathrm{Cqr}$ & $\mathrm{Cq} \delta_{1}$ \\
$\mathrm{pq}$ & - & - & $\mathrm{pqr}$ & $\mathrm{pq} \delta_{1}$ \\
$\mathrm{ABp}$ & - & - & $\mathrm{ABpr}$ & $\mathrm{ABp} \delta_{1}$ \\
$\mathrm{ACp}$ & - & - & $\mathrm{ACpr}$ & $\mathrm{ACp} \delta_{1}$ \\
$\mathrm{BCp}$ & - & - & $\mathrm{BCpr}$ & $\mathrm{BCp} \delta_{1}$ \\
$\mathrm{ABq}$ & - & - & $\mathrm{ABqr}$ & $\mathrm{ABq} \delta_{1}$ \\
$\mathrm{ACq}$ & - & - & $\mathrm{ACqr}$ & $\mathrm{ACq} \delta_{1}$ \\
$\mathrm{BCq}$ & - & - & $\mathrm{BCqr}$ & $\mathrm{BCq} \delta_{1}$ \\
$\mathrm{Apq}$ & - & - & $\mathrm{Apqr}$ & $\mathrm{Apq} \delta_{1}$ \\
$\mathrm{Bpq}$ & - & - & $\mathrm{Bpqr}$ & $\mathrm{Bpq} \delta_{1}$ \\
$\mathrm{Cpq}$ & - & - & $\mathrm{Cpqr}$ & $\mathrm{Cpq} \delta_{1}$ \\
$\mathrm{ABCp}$ & - & - & $\mathrm{ABCpr}$ & $\mathrm{ABCp} \delta_{1}$ \\
$\mathrm{ABCq}$ & - & - & $\mathrm{ABCqr}$ & $\mathrm{ABCq} \delta_{1}$ \\
$\mathrm{ABpq}$ & - & - & $\mathrm{ABpqr}$ & $\mathrm{ABpq} \delta_{1}$ \\
$\mathrm{ACpq}$ & - & - & $\mathrm{ACpqr}$ & $\mathrm{ACpq} \delta_{1}$ \\
$\mathrm{BCpq}$ & - & - & $\mathrm{BCpqr}$ & $\mathrm{BCpq} \delta_{1}$ \\
$\mathrm{ABCpq}$ & - & - & $\mathrm{ABCpqr}$ & $\mathrm{ABCpq} \delta_{1}$ \\
\hline & & & &
\end{tabular}

The search algorithm proceeds in a manner similar to that for the previous blocking 
schemes. Again, when sorting through the designs, one proceeds from left to right across the columns and from top to bottom within a column, while simultaneously choosing a single generator from each column. As before, DCSs are constructed and WLPs are compared with one another in the search for the design(s) with MA.

\subsection{Additional Optimality Criteria}

After searching through all $N_{1} \times N_{2}$ BFFSP design possibilities generated by the search-table, we may obtain many MA designs. In order to further differentiate between MA designs, we introduce the following six additonal optimality criteria:

(a) The number of clear main effects

(b) The number of clear two-factor interactions

(c) The number of clear SP main effects

(d) The number of clear SP two-factor interactions

(e) The number of clear SP main effects tested against WP error

(f) The number of clear SP two-factor interactions tested against WP error.

Each MA BFFSP design is assessed with respect to (a) - (f). Obviously, we would like an MA BFFSP design to have large values for criteria (a) - (d) and small values for criteria (e) and (f).

\subsection{A Catalog of MA BFFSP Designs}

The tables in Appendix B contain MA BFFSP designs constructed via pure WP blocking, separation and mixed blocking, respectively. All designs have between five and eleven factors and blocking variables (combined) and consist of either 8, 16 or 32 runs in either two, four or eight blocks. Each MA $2^{\left(n_{1}+n_{2}\right)-\left(k_{1}+k_{2}\right) \pm\left(b_{1}+b_{2}\right)}$ BFFSP design is abbreviated "Design $=n_{1}, n_{2} ; k_{1}, k_{2} ; b_{1}, b_{2}$ ". Within the tables, the designs are presented in ascending order of $n=n_{1}+n_{2}+b_{1}+b_{2}$, the total number of factors and blocking variables. Note that the WLPs are truncated at the last non-zero value. A-G and p-w are used to denote the WP and SP factors, respectively, with the last 
letter in each generator representing the added factor. The $i^{\text {th }}$ and $j^{\text {th }}$ WP blocking variable and separator are denoted by $\beta_{i}$ and $\delta_{j}$. In columns (a) - (f) we have included an assessment of each MA BFFSP design with respect to the six optimality criteria of Section 5.3. If an MA $2^{\left(n_{1}+n_{2}\right)-\left(k_{1}+k_{2}\right) \pm\left(b_{1}+b_{2}\right)}$ BFFSP design possesses a WLP identical to the design preceding it in the table, yet differs with respect to optimality criteria (a) - (f), we denote it by a "*" in the table. Note that any BFFSP design denoted by a "*" is superior with respect to at least one of the criteria (a) - (f), in comparison with the preceding design. This allows a practitioner to choose between MA BFFSP designs having identical WLPs, based upon the strength of the designs with regards to criteria (a) - (f).

Example 5.4.1 Consider a $2^{(4+4)-(0+3) \pm(1+0)}$ BFFSP design. Table B.3 in Appendix $B$ lists two such MA BFFSP designs, each with the same WLP. In order to choose between these similar designs one should assess the differences between the two designs with respect to the criteria in columns (a) - $(f)$. Using criterion ( $f)$, the design labeled "4,4;0,3;1,0" is better than design "*" because it has zero, rather than three, $S P$ 2fi's tested against WP error. However, using criterion (d), design "*” is better than design "4,4;0,3;1,0", because it possesses 10 rather than eight clear SP $2 f$ 's. The decision regarding which design to run may in part be motivated by the experimenter's preference for an MA BFFSP design that is optimal with respect to criteria (d) or (f).

\subsubsection{Overview of the MA Design Search Algorithm}

This section provides a brief overview of the structure of the program used to construct the MA BFFSP designs in Appendix B.

The program, "searchmixed.cpp", consists of a main body of code that calls upon four primary functions. These five program elements are now briefly described:

(1) Main Body of Program: This portion prompts the program user to enter all of the relevant variables. For example, $n_{1}, n_{2}, k_{1}, k_{2}, b_{1}$, and $b_{2}$ are all entered at this stage. The construction of the search-table is also completed at this stage for use in subsequent functions.

(2) Function "SearchDCSWLP": This function receives (from the main body of the program) the generators for each of the potential MA BFFSP designs. 
From these generators, the DCS of the design is constructed using modulus 2 arithmetic. The DCS is then passed back to the main body of the program to be used in subsequent functions.

(3) Function "BFFSPWLP": At this stage the WLP is calculated for the BFFSP design currently under consideration. The DCS used in the function's WLP calculations is received via function "SearchDCSWLP".

(4) Function "BFFSPCompareWLP": This function compares the WLP of the current design (as calculated by function "BFFSPWLP") with the WLPs of previous designs. If the current design has less aberration than previous designs the current DCS is retained and the others are discarded. If the current design has aberration equal to the currently optimal design then both designs are retained. Otherwise the design is discarded.

(5) Function "PrintBFFSPDesigns": A considerable number of calculations are performed by this function. It first receives all MA designs from function "BFFSPCompareWLP". Subsequently, all MA designs are assessed with regards to the six optimality criteria in Section 5.3. Those MA designs that are superior in at least one of the six criteria are retained. Finally, a representative MA design is printed from each group of MA designs that are superior in at least one of the six criteria.

The search algorithm and all other programmable calculations were implemented using Microsoft $t_{\circledast}$ Visual $C^{++}$6.0 Professional Edition. Computer hardware consisted of a Dimension ${ }^{T M} 8100$ Desktop Pentium 4 computer with a $1.40 \mathrm{GHz}$ CPU.

\subsubsection{A Note on the Incompleteness of the MA BFFSP De- sign Catalog}

If desired, implementation of the search-table algorithm via the program "searchmixed.cpp" will allow us to construct any 8, 16, or 32-run two-level BFFSP design. However, upon examination of the MA catalog it is evident that not all possible 8, 16 and 32-run MA BFFSP designs are listed - there are several reasons for this.

First, many BFFSP designs are impractical by our definition (Section 3.2.2) and therefore we have chosen to exclude these designs from the catalog. Second, ineligible 
designs (designs with $R<3$ ) are of little interest to an experimenter due to their poor estimation capacity and therefore are excluded from the catalog as well.

Additional reasons for not including other 8, 16 or 32-run MA BFFSP designs in Appendix B include:

1. If the design possesses a small number of clear SP main effects and 2fi's (for example, 16-run designs with pure WP blocking having $n_{1}+n_{2}+b_{1}+b_{2} \geq 11$; 16-run separated designs having $n_{1}+n_{2}+b_{1}+b_{2} \geq 11$ ).

2. If the design has a large number of clear SP main effects and 2fi's tested against WP error (for example, 32-run mixed designs with $n_{1}+n_{2}+b_{1}+b_{2} \geq 11$ ).

3. Time considerations - constructing BFFSP designs can be time intensive. Therefore, we do not include any designs with $n_{1}+n_{2}+b_{1}+b_{2}>11$. However, with this said, a practitioner may still request that an excluded $2^{\left(n_{1}+n_{2}\right)-\left(k_{1}+k_{2}\right) \pm\left(b_{1}+b_{2}\right)}$ design be constructed without the program being hindered in any way. 


\section{Chapter 6}

\section{Analysis of Variance for BFFSP Designs}

The analysis of variance (ANOVA) approach provides the experimenter with a versatile statistical tool for studying the relationship between a response variable and one or more (treatment) factors. The main task of an ANOVA is to quantify and evaluate the importance of possible sources of variation (factor effects, error terms) in an underlying linear model. Generally speaking, this is accomplished by forming a partition of the total sum of squares and degrees of freedom (df) associated with the response variable, $y$, into its component parts. ANOVA models allow for the independent variables to be both qualitative (for example, day of the week, process operator) and quantitative (for example, temperature, weight).

In this chapter we will examine the use of ANOVA models for assessing the magnitude of factor effects in various two-level designs. This is done so as to provide the practitioner with a statistical tool for ascertaining the important experimental factors when investigating some process. We begin by considering ANOVA concepts with application to $2^{k}$ full factorial designs eventually proceeding to ANOVA models for $2^{\left(n_{1}+n_{2}\right)-\left(k_{1}+k_{2}\right) \pm\left(b_{1}+b_{2}\right)}$ BFFSP designs.

\subsection{ANOVA Models for $2^{k}$ Designs}

To illustrate the general approach for the analysis of $2^{k}$ designs via an ANOVA model we summarize an example given by Montgomery (2001). 
Example 6.1.1 In this study the experimenter invesigated the effects of three factors (percent carbonation, operating pressure and line speed - each at two levels) on the fill height of a carbonated beverage. This resulted in a $2^{3}$ design being performed. In addition, the experimenter replicated the design twice so that 16 observations were obtained. The full factorial design matrix (with corresponding observations) is given in Figure 6.1. Percent carbonation, operating pressure and line speed are denoted by $A, B$ and $C$, respectively.

Figure 6.1: The Design Matrix for the Replicated $2^{3}$ Design.

$$
X=\left(\begin{array}{rrrrrrrr}
A & B & C & A B & A C & B C & A B C & y \\
- & - & - & + & + & + & - & -3 \\
+ & - & - & - & - & + & + & 0 \\
- & + & - & - & + & - & + & -1 \\
+ & + & - & + & - & - & - & 2 \\
- & - & + & + & - & - & + & -1 \\
+ & - & + & - & + & - & - & 2 \\
- & + & + & - & - & + & - & 1 \\
+ & + & + & + & + & + & + & 6 \\
- & - & - & + & + & + & - & -1 \\
+ & - & - & - & - & + & + & 1 \\
- & + & - & - & + & - & + & 0 \\
+ & + & - & + & - & - & - & 3 \\
- & - & + & + & - & - & + & 0 \\
+ & - & + & - & + & - & - & 1 \\
- & + & + & - & - & + & - & 1 \\
+ & + & + & + & + & + & + & 5
\end{array}\right)
$$

Sums of squares for estimated factorial effects are denoted by $S S_{\hat{c}}$ and are obtained by use of the equation,

$$
S S_{\hat{c}}=\frac{\left(\boldsymbol{x}_{c}^{\prime} \boldsymbol{y}\right)^{2}}{N}
$$


where $\boldsymbol{x}_{c}$ denotes the column of $+/$ - signs for a specific factor $c, N=r 2^{k}$ (the total number of runs) and $r$ signifies the number of times the design is replicated. For example, the sum of squares due to percent carbonation is given by

$$
\begin{aligned}
& S S_{\widehat{A}}=\frac{\left(\boldsymbol{x}_{A}^{\prime} \boldsymbol{y}\right)^{2}}{16} \\
& =\frac{1}{16}\left((-+\cdots-+)\left(\begin{array}{llll}
-3 & 0 \ldots 1 & 5
\end{array}\right)^{\prime}\right)^{2} \\
& =\frac{(24)^{2}}{16} \\
& =36.0 \text {. }
\end{aligned}
$$

Sums of squares for the effects $B, C, A B, A C, B C$, and $A B C$ are obtained in a similar fashion. The total sum of squares, $S S_{\text {Tot }}$, is calculated by taking the sum of the squares of the deviations of the individual observations from the overall mean, $\bar{y}$. This quantity is easily obtained by use of the computational form for $S S_{T o t}$, given by,

$$
S S_{T o t}=\sum_{i=1}^{2} \sum_{j=1}^{2} \sum_{l=1}^{2} \sum_{r=1}^{2} y_{i j l r}^{2}-\frac{y_{\ldots \ldots}^{2}}{N},
$$

where $i, j$ and $l$ denote the $i^{\text {th }}, j^{\text {th }}$ and $l^{\text {th }}$ levels of factors $A, B$ and $C$, respectively. The resulting ANOVA table is given in Table 6.1.

Note that the sum of squares due to error (unexplained variation in the response) may be obtained last, by subtraction, and is usually labeled SSE. If there had been no replication of the $2^{3}$ design SSE could not have been formally calculated. In such cases, sums of squares for higher-order interactions are often "pooled" together in order to obtain an estimate of SSE.

It is now useful to turn our attention to the three rightmost columns in Table 6.1. The third column in Table 6.1 displays the df associated with each source of variation in the ANOVA model. The df for any factorial effect is equal to the number of its treatment levels less one. Therefore, all factorial effects in a two-level design will possess $1 \mathrm{df}$. The total $d f$ for any two-level design is $N-1$, the total number of observations less one. Here the df for the lone error term is obtained by subtracting the total number of factorial effects, $2^{k}-1$, from $N-1$.

For each source of variation the fourth column displays the associated mean squares (MS). These are the sums of squares for each source of variation divided by their corresponding df. By dividing the MS of each effect with its corresponding 
Table 6.1: ANOVA for the Replicated $2^{3}$ Design in Example 6.1.1.

\begin{tabular}{lrrrr}
\hline \hline Source of & Sum of & \multicolumn{2}{c}{ Degrees of } & Mean \\
Variation & Squares & Freedom & Square & $F_{0}$ \\
\hline $\mathrm{A}$ & 36.00 & 1 & 36.00 & 57.60 \\
$\mathrm{~B}$ & 20.25 & 1 & 20.25 & 32.40 \\
$\mathrm{C}$ & 12.25 & 1 & 12.25 & 19.60 \\
$\mathrm{AB}$ & 2.25 & 1 & 2.25 & 3.60 \\
$\mathrm{AC}$ & 0.25 & 1 & 0.25 & 0.40 \\
$\mathrm{BC}$ & 1.00 & 1 & 1.00 & 1.60 \\
$\mathrm{ABC}$ & 1.00 & 1 & 1.00 & 1.60 \\
Error & 5.00 & 8 & 0.625 & \\
\hline \hline Design Total & 78.00 & 15 & & \\
\hline \hline
\end{tabular}

error term we form the ratio,

$$
F_{0}=\frac{S S_{\hat{c}} / d f_{\hat{c}}}{S S E / d f_{\text {Error }}}=\frac{S S_{\hat{c}} / 1}{S S E /\left((r-1) 2^{k}\right)}=\frac{M S_{\hat{c}}}{M S E} .
$$

Equation 6.3 is the test statistic for the null hypothesis $\left(H_{0}\right)$ that "the main effect or interaction, denoted by $\hat{c}$, exerts no statistically significant effect on the response". If $H_{0}$ is true, the ratio has the $F$ distribution with 1 and $(r-1) 2^{k} d f$ (Montgomery, 2001). If $H_{0}$ is false, then the expected value of $M S_{\hat{c}}$ is greater than the expected value of MSE. Therefore, under the alternative hypothesis $\left(H_{1}\right)$ that "the main effect or interaction exerts a statistically significant effect on the response", we reject $H_{0}$ for large values of Equation 6.3. Formally, we have an upper-tail critical region, where we reject $H_{0}$ if,

$$
F_{0}>F_{\alpha, 1,(r-1) 2^{k}}
$$

$\alpha$ being the level of significance for the hypothesis test. 


\subsection{ANOVA Basics for Other Two-level Designs}

Having been given an introduction to ANOVA concepts in the previous section we now briefly summarize some ANOVA issues associated with $2^{n-k} \mathrm{FF}$ and BFF designs.

Sums of squares calculations for factorial effects in $2^{n-k} \mathrm{FF}$ and BFF designs proceed in a similar fashion to that in the $2^{k}$ setting. Again, we denote sums of squares for estimated effects by $S S_{\hat{c}}$, and they are calculated via Equation 6.1, repeated below for the convenience of the reader:

$$
S S_{\hat{c}}=\frac{\left(\mathbf{x}_{c}^{\prime} \mathbf{y}\right)^{2}}{N} .
$$

Note that in the $2^{n-k} \mathrm{FF}$ and BFF setting $N=r 2^{n-k}$.

If we wish to estimate a main effect or interaction we change the denominator in Equation 2.1 to reflect the number of experimental runs in FF and BFF designs. As a result of the fractionation, Equation 2.1 becomes,

$$
\hat{c}=\frac{\mathbf{x}_{\mathbf{c}}^{\prime} \mathbf{y}}{r 2^{n-k-1}} .
$$

If a BFF design is run (thereby confounding higher-order interactions with block effects) the calculation of sums of squares for blocks is straightforward-simply find the sums of squares for those effects confounded with blocks. For example, suppose we wish to run a $2^{8-3}$ design in $2^{2}=4$ blocks, using blocking variable generators $\beta_{1}=A B E$ and $\beta_{2}=A B H$. This implies that we will have $3 \mathrm{df}$ for blocks, the third $\mathrm{df}$ accounted for by the product, $\beta_{1} \beta_{2}=E H$. By totaling the sums of squares of the confounded effects $\left(S S_{\widehat{A B E}}+S S_{\widehat{A B H}}+S S_{\widehat{E H}}\right)$ we will then have found $S S_{\text {Blocks }}$ for the $2^{8-3}$ BFF design.

Formation and interpretation of ANOVA tables proceeds in a manner analagous to that in Section 6.1. Montgomery (2001) and Cochran and Cox (1957) provide modern and classical references, respectively, to the topic of ANOVA in the two-level design setting. These texts present ANOVA models for virtually all "introductory" experimental designs. 


\subsection{ANOVA Models for Two-level FFSP Designs}

Having presented the basic concepts of ANOVA models we now enter the more complicated arena of ANOVA for the split-plot setting. In this section, examples will be given illustrating ANOVA models for two $2^{\left(n_{1}+n_{2}\right)-\left(k_{1}+k_{2}\right)}$ FFSP designs. This overview is motivated by examples given in Bingham and Sitter (2001) and Montgomery (2001). We wish to stress that despite the fact that the ANOVA results in this section are conceptually well established (Kempthorne, 1952; Hinkelmann and Kempthorne, 1994) it is rare (see references in previous sentence) to find them outlined in their general form.

(For the interested reader, a thorough discussion of ANOVA for multi-level splitplot designs may be found in Hinkelmann and Kempthorne (1994). This text provides considerable insight into the split-plot setting outside of the two-level factorial framework in which we are now entrenched. Some results in the following sections are motivated by this text.)

\subsubsection{Two Approaches}

Two slightly different approaches may be taken when constructing an ANOVA model for a $2^{\left(n_{1}+n_{2}\right)-\left(k_{1}+k_{2}\right)}$ FFSP design. One approach is implicitly suggested by Bingham and Sitter (2001). In this article they provide an example of a $2^{(3+2)-(0+1)}$ FFSP design replicated four times. Their analysis description states that the df for WP error is $(r-1) 2^{n_{1}-k_{1}}$. (Therefore, this implies that there will be $(4-1) 2^{3-0}=24 \mathrm{df}$ for WP error.) They also state that the $\mathrm{df}$ for SP error is $(r-1)\left(2^{\left(n_{1}+n_{2}\right)-\left(k_{1}+k_{2}\right)}-2^{n_{1}-k_{1}}\right)$. From this we may infer the general ANOVA model, as displayed in Table 6.2.

Similarly, Montgomery (2001) provides an example of a replicated $2^{(2+2)-(0+0)}$ split-plot design. In the subsequent analysis, df for replications is separated from the df for WP error, implying the general ANOVA model outlined in Table 6.3. If it is not expected that there will exist statistically significant differences between replications we envision similar results from both ANOVA models. In this case we may think of the WP portion to be run as a completely randomized design (CRD), thus performing an ANOVA on the WP observations (averaged over the SP treatment combinations) using the model of Table 6.2. If it is thought that replications will account for a significant portion of the WP error, it is wise to separately calculate the 
Table 6.2: An ANOVA Model for the $2^{\left(n_{1}+n_{2}\right)-\left(k_{1}+k_{2}\right)}$ FFSP Design.

\begin{tabular}{lcc}
\hline \hline \multicolumn{2}{l}{ Source of Variation } & Degrees of Freedom \\
\hline WP & & \\
& Effects & $2^{n_{1}-k_{1}}-1$ \\
& Error & $(r-1) 2^{n_{1}-k_{1}}$ \\
\hline WP Total & & $r 2^{n_{1}-k_{1}}-1$ \\
\hline SP & & \\
& Effects & $2^{\left(n_{1}+n_{2}\right)-\left(k_{1}+k_{2}\right)}-2^{n_{1}-k_{1}}$ \\
& Error & $(r-1)\left(2^{\left(n_{1}+n_{2}\right)-\left(k_{1}+k_{2}\right)}-2^{n_{1}-k_{1}}\right)$ \\
\hline \hline Design Total & & $r 2^{\left(n_{1}+n_{2}\right)-\left(k_{1}+k_{2}\right)}-1$ \\
\hline \hline
\end{tabular}

sum of squares for replications $\left(S S_{\text {Reps }}\right)$, thereby following the outline in Table 6.3. A computational formula for finding $S S_{\text {Reps }}$ is given by

$$
S S_{R e p s}=\sum_{h=1}^{T} \frac{R_{h}^{2}}{2^{\left(n_{1}+n_{2}\right)-\left(k_{1}+k_{2}\right)}}-\frac{y_{T o t}^{2}}{N},
$$

where $R_{h}$ equals the sum of the observations in the $h^{\text {th }}$ replicate, $y_{\text {Tot }}^{2}$ equals the square of the total of all the observations and $N=r 2^{\left(n_{1}+n_{2}\right)-\left(k_{1}+k_{2}\right)}$.

The SP portion of the design is typically viewed as a randomized block design (RBD) with the "blocks" being the $r 2^{n_{1}-k_{1}}$ WP treatment combinations. To account for the number of $\mathrm{df}$ for SP effects $\left(2^{\left(n_{1}+n_{2}\right)-\left(k_{1}+k_{2}\right)}-2^{n_{1}-k_{1}}\right)$ we note that this is the number of main effects and interactions involving at least one SP factor that are not aliased with WP main effects or interactions involving only WP factors.

It is worthwhile noting that when split-plot designs are run outside of the twolevel factorial structure, we encounter other variations (besides the CRD and RBD formats) by which one may need to analyze the WP and SP portions. Again, the text by Hinkelmann and Kempthorne (1994) provides an informative discussion regarding this topic. 
Table 6.3: An Alternate ANOVA Model for the $2^{\left(n_{1}+n_{2}\right)-\left(k_{1}+k_{2}\right)}$ FFSP Design.

\begin{tabular}{lcc}
\hline \hline \multicolumn{1}{l}{ Source of Variation } & Degrees of Freedom \\
\hline Reps & & $r-1$ \\
\hline WP & & $2^{n_{1}-k_{1}}-1$ \\
& Effects & $(r-1)\left(2^{n_{1}-k_{1}}-1\right)$ \\
& Error & $r\left(2^{n_{1}-k_{1}}-1\right)$ \\
\hline WP Total & & \\
\hline SP & Effects & $2^{\left(n_{1}+n_{2}\right)-\left(k_{1}+k_{2}\right)}-2^{n_{1}-k_{1}}$ \\
& Error & $(r-1)\left(2^{\left(n_{1}+n_{2}\right)-\left(k_{1}+k_{2}\right)}-2^{n_{1}-k_{1}}\right)$ \\
\hline \hline Design Total & & $r 2^{\left(n_{1}+n_{2}\right)-\left(k_{1}+k_{2}\right)}-1$ \\
\hline \hline
\end{tabular}

\subsection{ANOVA for Unreplicated BFFSP Designs}

At this point we may begin discussion regarding ANOVA models for BFFSP designs. In this section we intend to concentrate our efforts upon the "simpler" unreplicated $2^{\left(n_{1}+n_{2}\right)-\left(k_{1}+k_{2}\right) \pm\left(b_{1}+b_{2}\right)}$ BFFSP design scenario. Aside from the simplicity of this setting we shall see in this section that there are additional justifications for this initial focus.

\subsubsection{Some Comments Regarding Unreplicated Designs}

One reason for the relevancy of unreplicated designs is due to the prohibitive size of two-level designs for even a "small" number of factors. For example, if $\left(n_{1}+n_{2}\right)-$ $\left(k_{1}+k_{2}\right) \geq 5$ we are faced with an experiment consisting of at least $2^{5}=32$ runswith just one replicate. Replicating a design of this magnitude only compounds the size problem in terms of the strain on an experimenter's resources (time, money, personnel).

A drawback of unreplicated designs is that they provide no estimate of WP or SP error. A possible solution to this problem is to assume that some (if not all) higher-order interactions are negligible thereby allowing one to combine their MS to 
estimate the two error terms. For split-plot designs, higher-order WP effects would be pooled in order to form $M S E_{\widehat{W P}}$, an estimate of $E\left[M S E_{W P}\right]$. Likewise, higher-order SP effects would be combined so as to form $M S E_{\widehat{S P}}$, an estimate of $E\left[M S E_{S P}\right]$. This method of pooling MS is not an unreasonable solution given our assumption of neglibility of effects of order three and higher. If one cannot assume the negligibility of certain higher-order effects, the experimenter should consider the use of normal probability plots of the effect estimates. For FF designs, Daniel (1959) provides the classical reference to this topic and most current texts on experimental design devote some discussion to this graphical tool. For FFSP designs, two normal probability plots should be constructed, one for the WP effects and one for the SP effects (Box and Jones, 1992; Bingham and Sitter, 2001).

\subsubsection{The ANOVA Model for Pure WP Blocking}

Table 6.4 displays the general ANOVA model for BFFSP designs in which blocks are generated solely by WP factors. This model contains several noteworthy characteristics. First, recall that for $b_{1}$ blocking variables we will have $2^{b_{1}}$ blocks. This explains the $2^{b_{1}}-1 \mathrm{df}$ reserved for WP effects confounded with blocks. Second, following the discussion in Section 6.2, $S S_{\text {Blocks }}$ is found by totaling the sums of squares of all effects confounded with blocks. Third, one notes that sums of squares calculations for all effects will follow in the same vein as Equation 6.1 but with $N=2^{\left(n_{1}+n_{2}\right)-\left(k_{1}+k_{2}\right) \pm\left(b_{1}+0\right)}=2^{\left(n_{1}+n_{2}\right)-\left(k_{1}+k_{2}\right)}$.

Fourthly, since we are assuming that the BFFSP design is not replicated we have no formal estimate of error (signified by the solid line "___ in the ANOVA table). As previously mentioned, we could combine MS of higher-order effects to obtain the estimates $M S E_{\overparen{W P}}$ and $M S E_{\widehat{S P}}$. (Since we are assuming throughout this research the negligibility of effects of order three and higher, this pooling of MS, though not ideal, is not of serious concern.) Upon forming estimates of the respective error terms, one may perform tests of significance for the main effects and 2fi's. Recall that in a test of significance we first need to construct the test statistic, $F_{0}$, a ratio of MS, (Section 6.1). For example, if one wished to test the significance of an estimated WP 2fi, say $\widehat{A B}$, we would form the test statistic,

$$
F_{0}=\frac{M S_{\overparen{A B}}}{M S E_{\overparen{W P}}}
$$


Table 6.4: The ANOVA Model for the $2^{\left(n_{1}+n_{2}\right)-\left(k_{1}+k_{2}\right) \pm\left(b_{1}+0\right)}$ BFFSP Design.

\begin{tabular}{llc}
\hline \hline \multicolumn{2}{c}{ Source of Variation } & Degrees of Freedom \\
\hline Blocks & Pure WP Blocks & $2^{b_{1}}-1$ \\
\hline WP & Effects & $2^{n_{1}-k_{1}}-2^{b_{1}}$ \\
& Error & - \\
\hline WP Total & & $2^{n_{1-k_{1}}}-2^{b_{1}}$ \\
\hline SP & & \\
& Effects & $2^{\left(n_{1}+n_{2}\right)-\left(k_{1}+k_{2}\right)}-2^{n_{1}-k_{1}}$ \\
& Error & \\
\hline \hline Design Total & & $2^{\left(n_{1}+n_{2}\right)-\left(k_{1}+k_{2}\right)}-1$ \\
\hline \hline
\end{tabular}

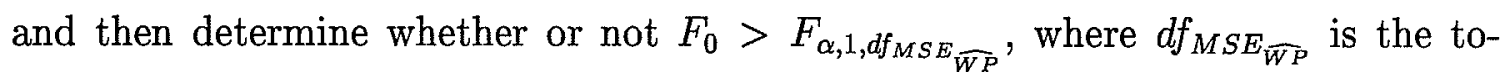
tal number of higher-order WP effects pooled together to form the error estimate, $M S E_{\widehat{W P}}$.

Fifthly, from Table 6.4 we see that there are $2^{\left(n_{1}+n_{2}\right)-\left(k_{1}+k_{2}\right)}-2^{n_{1}-k_{1}}$ df reserved for SP effects. These effects are all those main effects and interactions, comprised of at least one SP factor, that are not aliased with WP main effects, pure WP blocking variables or interactions involving only WP factors and/or pure WP blocking variables. In testing the significance of an estimated SP effect, say the 2 fi $\widehat{A q}$, we would form the test statistic,

$$
F_{0}=\frac{M S_{\overparen{A q}}}{M S E_{\overparen{S P}}}
$$

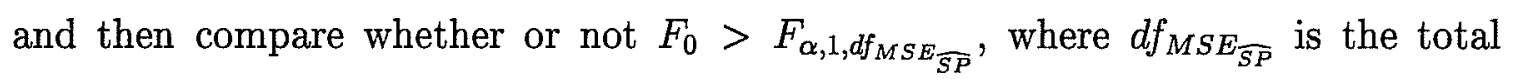
number of higher-order SP effects pooled together to form the estimate, $M S E_{\widehat{S P}}$.

Finally, suppose an experimenter is interested in testing for the statistical significance of block effects. From the ANOVA table, one may be inclined to form the ratio

$$
F_{0}=\frac{M S_{B l o c k s}}{M S E_{\overparen{W P}}}
$$




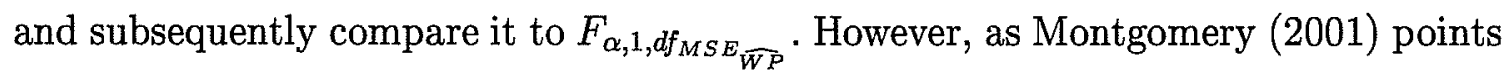
out, in the context of randomized complete block designs (RCBDs), randomization has been applied only to the treatments within blocks; therefore, the blocks place a restriction on randomization. The impact of this randomization restriction is debated in the literature. Box, Hunter and Hunter (1978) say that an $F$ test for the comparison of block means (effects) is meaningful provided the errors are normally and identically distributed with constant variance; that is, if the errors are $\operatorname{NID}\left(0, \sigma^{2}\right)$. Anderson and McLean (1974) say that regardless of the distribution of the errors, this test is still meaningless. Montgomery (2001) agrees in principle with Box, Hunter and Hunter (1978) but notes that the assumption of normality for the error terms is often questionable, so "an exact $F$ test on the equality of block means is not a good general practice". Although the discussion given in the preceding references is with regards to RCBDs and other "simple" two-level designs possessing blocks, these arguments carry over to the BFFSP setting since randomization of the treatment combinations is performed within blocks as well. Consequently, in subsequent analyses for unreplicated designs we will exclude formal $F$ tests for the comparison of block means. We shall see in later sections that in the presence of design replication we may introduce an appropriate formal test for block effects.

Example 6.4.1 To illustrate the preceding model, we turn again to the $2^{(3+3)-(0+1) \pm(1+0)}$ BFFSP design (as in Section 3.1.1) having DCS $I=A B C \beta_{1}=A B p q r=C p q r \beta_{1}$. $B y$ displaying the design's alias structure in Table 6.5, we indicate the appropriate breakdown of the df for all sources of variation. Note that for the sake of brevity, treatment $\times$ block interactions (which we assume to be negligible) have been excluded from the displayed alias structure.

The total df for blocks is $2^{1}-1=1$, the total df for WP effects is $2^{(3-0)}-2^{1}=6$ and the total $d f$ for $S P$ effects is $2^{(3+3)-(0+1)}-2^{(3-0)}=24$, which is in agreement with Table 6.4.

\subsubsection{The ANOVA Model for Blocking via Separation}

Table 6.6 displays the general ANOVA model for the BFFSP design in which blocks are generated via separation. The creation of $b_{2}$ separators will result in $2^{b_{2}}$ blocks being formed, so $2^{b_{2}}-1 \mathrm{df}$ are reserved for block effects. Total df for SP effects is 
Table 6.5: Alias Structure of the $2^{(3+3)-(0+1) \pm(1+0)}$ BFFSP Design in Example 6.4.1.

\begin{tabular}{lll}
\hline \hline & \multicolumn{1}{c}{ Alias Chain } & $\mathrm{df}$ \\
\hline Blocks & & \\
& $\beta_{1}=A B C=C p q r$ & 1 \\
\hline WP Effects & & \\
& $A=B p q r$ & 1 \\
$B=A p q r$ & 1 \\
$C=A B C p q r$ & 1 \\
$A B=p q r$ & 1 \\
$A C=B C p q r$ & 1 \\
$B C=A C p q r$ & 1 \\
\hline & & Total wP df
\end{tabular}

\section{SP Effects}

\begin{tabular}{lll}
$p=A B q r$, & $A B p=q r$ & 2 \\
$q=A B p r$, & $A C p=B C q r$ & 2 \\
$r=A B p q$, & $B C p=A C q r$ & 2 \\
$A p=B q r$, & $A B C p=C q r$ & 2 \\
$B p=A q r$, & $A B q=p r$ & 2 \\
$C p=A B C q r$, & $A C q=B C p r$ & 2 \\
$A q=B p r$, & $B C q=A C p r$ & 2 \\
$B q=A p r$, & $A B C q=C p r$ & 2 \\
$C q=A B C p r$, & $A B r=p q$ & 2 \\
$A r=B p q$, & $A C r=B C p q$ & 2 \\
$B r=A p q$, & $B C r=A C p q$ & 2 \\
$C r=A B C p q$, & $A B C r=C p q$ & 2 \\
\hline \hline
\end{tabular}

then $2^{\left(n_{1}+n_{2}\right)-\left(k_{1}+k_{2}\right)}-2^{n_{1}-k_{1}+b_{2}}$, the number of design points less the number of block and WP effects.

The variation due to the separators has been delineated from the SP portion of the 
Table 6.6: The ANOVA Model for the $2^{\left(n_{1}+n_{2}\right)-\left(k_{1}+k_{2}\right) \pm\left(0+b_{2}\right)}$ BFFSP Design.

\begin{tabular}{lcc}
\hline \hline \multicolumn{2}{c}{ Source of Variation } & Degrees of Freedom \\
\hline Blocks & Separators & $2^{b_{2}}-1$ \\
\hline WP & Effects & $2^{n_{1}-k_{1}+b_{2}}-2^{b_{2}}$ \\
& Error & - \\
\hline WP Total & & $2^{n_{1}-k_{1}+b_{2}}-2^{b_{2}}$ \\
\hline SP & Effects & $2^{\left(n_{1}+n_{2}\right)-\left(k_{1}+k_{2}\right)}-2^{n_{1}-k_{1}+b_{2}}$ \\
& Error & \\
\hline \hline Design Total & & $2^{\left(n_{1}+n_{2}\right)-\left(k_{1}+k_{2}\right)}-1$ \\
\hline \hline
\end{tabular}

ANOVA table. This is done to emphasize that although the separators are blocking variables containing at least one SP factor, we do not test for their significance by use of the estimate, $M S E_{\widehat{S P}}$, in the test statistic, $F_{0}$. (Deriving the variances of the separators by the methods introduced in Chapter 4 would confirm this.) Of course, we do not wish to test for the significance of block effects anyways - this follows from the comments made in Section 6.4.2 regarding the inappropriateness of tests of significance for block means in the unreplicated design setting. (Section 6.5 will demonstrate how one may, in the presence of replication, correctly test for the significance of block effects.)

Other calculations, including tests of significance for effects not confounded with separators proceed in a manner analogous to that for designs with pure WP blocking variables.

The most noteworthy change to our ANOVA model, as a result of separation, occurs in the total number of df available at the WP level of the design. For each additional separator we see a two-fold increase in the number of distinct WP runs-a type of pseudo-replication (as explained in Chapters 3 and 4). We recall that this pseudo-replication provides the experimenter with increased precision when calcu- 
lating the variance of effects that are to be tested against the WP error. In Table 6.6 the benefit of separation is seen as an increase in available df for WP effects. The cost of this WP run replication is seen at the SP level of the design. Specifically, at the SP level each additional separator causes a decrease, by a factor of two, in the number of SP treatment combinations associated with each WP treatment combination. In the SP portion of the ANOVA table, the net effect of separation is to reduce the available df for SP effects by a factor of $2^{b_{2}}$. Note that forming "too many" blocks via separation will result in there being no SP effects. This will occur when $b_{2}=n_{2}-k_{2}$, implying that we have $2^{\left(n_{1}+n_{2}\right)-\left(k_{1}+k_{2}\right)}-2^{n_{1}-k_{1}+b_{2}}=2^{\left(n_{1}+n_{2}\right)-\left(k_{1}+k_{2}\right)}-2^{n_{1}-k_{1}+n_{2}-k_{2}}=0$ SP effects. Of course, in the case where $b_{2}=n_{2}-k_{2}$ an impractical design will result (see Section 3.2.2). Recall that we do not consider an impractical design to be a viable experimental option.

Example 6.4.2 Consider the $2^{(3+3)-(0+1) \pm(0+1)}$ BFFSP design having DCS $I=$ $A B C p r=A B q \delta_{1}=C p q r \delta_{1}$ (this design was first introduced in Chapter 3). Table 6.7 contains the design's alias structure, thereby exhibiting the proper distribution of $d f$ between the sources of variation. Note that some of the treatment $\times$ block interactions have been included in the displayed alias structure. We have only included treatment $\times$ block interactions in those alias chains for which it would otherwise be ambiguous as to the design status (WP or SP) of the other effects in the chain. The implication in Table 6.7 is that any effect aliased with a treatment $\times$ block interaction is in fact a WP effect. That is, in any tests of significance, the MS of these aliased effects will be tested against $M S E_{\overparen{W P}}$. (This can be confirmed by deriving the variance forms of treatment $\times$ block interactions using the methods of Chapter 4.)

The total df for blocks (separators) is $2^{1}-1=1$, the total df for WP effects is $2^{(3-0+1)}-2^{1}=14$ and the total df for SP effects is $2^{(3+3)-(0+1)}-2^{(3-0+1)}=16$, which is in agreement with Table 6.6.

\subsubsection{The ANOVA Model for Mixed Blocking}

In Table 6.8 we present the general ANOVA model for unreplicated BFFSP designs possessing mixed blocking. In essence, this model serves as an amalgamation of the models presented in Tables 6.4 and 6.6.

For mixed blocking there are $2^{b_{1}+b_{2}}-1 \mathrm{df}$ associated with blocks. We see that $2^{b_{1}}-1 \mathrm{df}$ are reserved for pure WP blocking variables, $2^{b_{2}}-1 \mathrm{df}$ are reserved for 
Table 6.7: Alias Structure of the $2^{(3+3)-(0+1) \pm(0+1)}$ BFFSP Design in Example 6.4.2.

\begin{tabular}{|c|c|c|c|c|}
\hline & Alias Chai & & & $\mathrm{df}$ \\
\hline \multicolumn{5}{|l|}{ Separators } \\
\hline & \multicolumn{3}{|l|}{$\delta_{1}=C p q r=A B q$} & 1 \\
\hline \multicolumn{5}{|l|}{ WP Effects } \\
\hline & $A=B C p r$ & $q=A B C p q r=A B \delta_{1}$ & & 2 \\
\hline & $B=A C p r$ & $A q=B C p q r=B \delta_{1}$ & & 2 \\
\hline & $C=A B p r$ & $B q=A C p q r=A \delta_{1}$ & & 2 \\
\hline & $A B=C p r$ & $C q=A B p q r=A B C \delta_{1}$ & & 2 \\
\hline & $A C=B p r$ & $A C q=B p q r=B C \delta_{1}$ & & 2 \\
\hline & $B C=A p r$ & $B C q=A p q r=A C \delta_{1}$ & & 2 \\
\hline & $A B C=p r$ & $A B C q=p q r=C \delta_{1}$ & & 2 \\
\hline & & & Total WP df & 14 \\
\hline \multicolumn{5}{|l|}{ SP Effects } \\
\hline & $p=A B C r$ & $p q=A B C q r$ & & 2 \\
\hline & $r=A B C p$ & $A q r=B C p q$ & & 2 \\
\hline & $A p=B C r$ & $B q r=A C p q$ & & 2 \\
\hline & $B p=A C r$ & $C q r=A B p q$ & & 2 \\
\hline & $C p=A B r$ & $A B q r=C p q$ & & 2 \\
\hline & $A r=B C p$ & $A B r=p q$ & & 2 \\
\hline & $B r=A C p$ & $A C q r=B p q$ & & 2 \\
\hline & $C r=A B p$ & $B C q r=A p q$ & & 2 \\
\hline & & & Total SP df & 16 \\
\hline
\end{tabular}

separators and the remaining $\left(2^{b_{1}}-1\right)\left(2^{b_{2}}-1\right)$ df are associated with those blocking variables generated by the product of the pure WP blocking variables and separators. Again, for unreplicated BFFSP designs with mixed blocking we suggest that one not test for the significance of block effects. In subsequent sections, when design replicates are considered, we will present a better approach for testing block means. 
Table 6.8: The ANOVA Model for the $2^{\left(n_{1}+n_{2}\right)-\left(k_{1}+k_{2}\right) \pm\left(b_{1}+b_{2}\right)}$ BFFSP Design.

\begin{tabular}{lll}
\hline \hline & Source of Variation & \multicolumn{1}{c}{ Degrees of Freedom } \\
\hline Blocks & & \\
& Pure WP Blocks & $2^{b_{1}}-1$ \\
& Separators & $2^{b_{2}}-1$ \\
& Pure WP Blocks $\times$ Separators & $\left(2^{b_{1}}-1\right)\left(2^{b_{2}}-1\right)$ \\
\hline Block Total & & $2^{b_{1}+b_{2}}-1$ \\
\hline WP & & \\
& Effects & $2^{n_{1}-k_{1}+b_{2}}-2^{b_{1}+b_{2}}$ \\
& Error & - \\
\hline WP Total & & $2^{n_{1}-k_{1}+b_{2}}-2^{b_{1}+b_{2}}$ \\
\hline SP & & \\
& Effects & $2^{\left(n_{1}+n_{2}\right)-\left(k_{1}+k_{2}\right)}-2^{n_{1}-k_{1}+b_{2}}$ \\
& Error & \\
\hline \hline Design Total & & $2^{\left(n_{1}+n_{2}\right)-\left(k_{1}+k_{2}\right)}-1$ \\
\hline \hline
\end{tabular}

Again, recall that for each additional separator we see a two-fold increase in the number of distinct WP runs. In Table 6.8 the benefit of separation is seen as an increase in available df for WP effects. The cost of this WP run replication is seen at the SP level of the design. Specifically, at the SP level each additional separator causes a decrease, by a factor of two, in the number of SP treatment combinations associated with each WP treatment combination. In the SP portion of the ANOVA table the net effect of separation is to reduce the available df for SP effects by a factor of $2^{b_{2}}$.

Example 6.4.3 The alias structure for the $2^{(3+3)-(0+1) \pm(1+1)}$ BFFSP design with $D C S I=A B q r=A B C \beta_{1}=B C p q \delta_{1}=C q r \beta_{1}=A C p r \delta_{1}=B p r \beta_{1} \delta_{1}=A p q \beta_{1} \delta_{1}$, is presented in Table 6.9. The resulting breakdown of $d f$ for each source of variation is in accordance with the general ANOVA model in Table 6.8. As in Table 6.7, note that some of the treatment $\times$ block interactions have been included in the displayed alias structure. We have only included treatment $\times$ block interactions in those alias chains 
for which it would otherwise be ambiguous as to the design status (WP or SP) of the other effects in the chain. The implication in Table 6.9 is that any effect aliased with a treatment $\times$ block interaction is in fact a WP effect. That is, in any tests of significance, the $M S$ of these aliased effects will be tested against $M S E_{\widehat{W P}}$. (This can be confirmed by deriving the variance forms of treatment $\times$ block interactions using the methods of Chapter 4.)

The total df for block effects is $2^{1+1}-1=3$, the total df for WP effects is $2^{(3-0+1)}-2^{(1+1)}=12$ and the total df for SP effects is $2^{(3+3)-(0+1)}-2^{(3-0+1)}=16$, which is in agreement with Table 6.8.

\subsection{ANOVA for Replicated BFFSP Designs}

On occasion, an experimenter may have adequate resources that allow design replicates to be run. This occurrence is sufficiently commonplace, hence we devote this section to considering the impact of replication upon the analysis of $2^{\left(n_{1}+n_{2}\right)-\left(k_{1}+k_{2}\right) \pm\left(b_{1}+b_{2}\right)}$ BFFSP designs.

Design replication causes the complexity of ANOVA models to increase. We will use the results of Section 6.4 as a stepping stone for developing this more technical setting.

\subsubsection{The ANOVA Model for Pure WP Blocking: With Repli- cation}

Replicating a $2^{\left(n_{1}+n_{2}\right)-\left(k_{1}+k_{2}\right) \pm\left(b_{1}+0\right)}$ BFFSP design implies that $r 2^{\left(n_{1}+n_{2}\right)-\left(k_{1}+k_{2}\right)}$ treatment combinations will be run. Therefore, $r 2^{\left(n_{1}+n_{2}\right)-\left(k_{1}+k_{2}\right)}-1 \mathrm{df}$ must be accounted for in the ANOVA model (see Table 6.10).

Given that there are $2^{b_{1}}$ blocks per replicate this implies that there will be a total of $r 2^{b_{1}}$ blocks in the design. Therefore, the model will have $r 2^{b_{1}}-1 \mathrm{df}$ reserved for block effects. In this model we extend the method described by Cochran and Cox (1957) for distributing the $\mathrm{df}$ for blocks and replicates in a replicated full factorial two-level design. In this reference, the authors explain that block df can be thought to consist of the $\mathrm{df}$ for replicates, factorial effects confounded with blocks and the interaction, replicates $\times$ blocks. Therefore, for a $2^{\left(n_{1}+n_{2}\right)-\left(k_{1}+k_{2}\right) \pm\left(b_{1}+0\right)}$ BFFSP design, 
Table 6.9: Alias Structure of the $2^{(3+3)-(0+1) \pm(1+1)}$ BFFSP Design in Example 6.4.3.

\begin{tabular}{llc}
\hline \hline Alias Chain & $\mathrm{df}$ \\
\hline Blocks & & \\
& $\beta_{1}=C q r=A B C$ & 1 \\
$\delta_{1}=A C p r=B C p q$ & & 1 \\
$\beta_{1} \delta_{1}=B p r=A p q$ & & 1 \\
\hline & Total Block df & 3
\end{tabular}

WP Effects

\begin{tabular}{|c|c|c|c|}
\hline & $A=B q r$ & $p q=A B p r=B C \delta_{1}=A \beta_{1} \delta_{1}$ & 2 \\
\hline & $B=A q r$ & $p r=A B p q=A C \delta_{1}=B \beta_{1} \delta_{1}$ & 2 \\
\hline & $C=A B C q r$ & $A p r=B p q=C \delta_{1}=A B \beta_{1} \delta_{1}$ & 2 \\
\hline & $A B=q r$ & $C p r=A B C p q=A \delta_{1}=B C \beta_{1} \delta_{1}$ & 2 \\
\hline & $A C=B C q r$ & $B C p r=A C p q=A B \delta_{1}=C \beta_{1} \delta_{1}$ & 2 \\
\hline & $B C=A C q r$ & $A B C p r=C p q=B \delta_{1}=A C \beta_{1} \delta_{1}$ & 2 \\
\hline \multirow{11}{*}{ SP Effects } & & Total WP df & 12 \\
\hline & & & \\
\hline & $p=A B p q r$ & $C q=A B C r$ & 2 \\
\hline & $q=A B r$ & $C r=A B C q$ & 2 \\
\hline & $r=A B q$ & $A B p=p q r$ & 2 \\
\hline & $A p=B p q r$ & $A C p=B C p q r$ & 2 \\
\hline & $B p=A p q r$ & $B C p=A C p q r$ & 2 \\
\hline & $C p=A B C p q r$ & $A B C p=C p q r$ & 2 \\
\hline & $A q=B r$ & $A C q=B C r$ & 2 \\
\hline & $B q=A r$ & $B C q=A C r$ & 2 \\
\hline & & Total SP df & 16 \\
\hline
\end{tabular}

we conclude that there are $r-1 \mathrm{df}$ for replicates, $2^{b_{1}}-1 \mathrm{df}$ for effects confounded with blocks and $(r-1)\left(2^{b_{1}}-1\right)$ df for replicates $\times$ blocks. These df quantities sum to $r 2^{b_{1}}-1$ which is required.

The interaction, replicates $\times$ blocks, can be thought of as the error for which 
Table 6.10: ANOVA Model for a Replicated $2^{\left(n_{1}+n_{2}\right)-\left(k_{1}+k_{2}\right) \pm\left(b_{1}+0\right)}$ BFFSP Design.

\begin{tabular}{lll}
\hline \hline \multicolumn{1}{c}{ Source of Variation } & \multicolumn{1}{c}{ Degrees of Freedom } \\
\hline Blocks & & \\
& Replicates & $r-1$ \\
& $\begin{array}{l}\text { Pure WP Blocks } \\
\text { Error }\end{array}$ & $2^{b_{1}}-1$ \\
& $(r-1)\left(2^{b_{1}}-1\right)$ \\
& & $r 2^{b_{1}}-1$ \\
\hline Block Total & & \\
\hline WP & Effects & $2^{n_{1}-k_{1}}-2^{b_{1}}$ \\
& Error & $(r-1)\left(2^{n_{1}-k_{1}}-2^{b_{1}}\right)$ \\
\hline WP Total & & $r\left(2^{n_{1}-k_{1}}-2^{b_{1}}\right)$ \\
\hline SP & & \\
& Effects & $2^{\left(n_{1}+n_{2}\right)-\left(k_{1}+k_{2}\right)}-2^{n_{1}-k_{1}}$ \\
& Error & $(r-1)\left(2^{\left(n_{1}+n_{2}\right)-\left(k_{1}+k_{2}\right)}-2^{n_{1}-k_{1}}\right)$ \\
\hline \hline Design Total & & $r 2^{\left(n_{1}+n_{2}\right)-\left(k_{1}+k_{2}\right)}-1$ \\
\hline \hline
\end{tabular}

the MS of those effects confounded with blocks are compared to. Consequently, in the presence of replication, we are now able to correctly test for the significance of block means; recall that this was not possible for unreplicated BFFSP designs. However, one should note that if the df for replicates $\times$ blocks is small, this test will be insensitive in detecting block differences (Cochran and Cox, 1957).

Despite the ability to test for block effects the practitioner will likely choose not to perform such tests. From a practical standpoint, the reason for blocking in the first place is because of the desire to control for variation in the response due to nonhomogeneous conditions in the experiment. Any test for block effects would most likely only serve to confirm their significance.

The design replicates now allow the experimenter to calculate the "true" MSE estimates $\left(M S E_{W P}\right.$ and $\left.M S E_{S P}\right)$ of the WP and SP error terms $\left(\sigma_{W P}^{2}\right.$ and $\left.\sigma_{S P}^{2}\right)$. No longer do we have to pool negligible higher-order interactions to form estimates of $E\left[M S E_{W P}\right]$ and $E\left[M S E_{S P}\right]$ as we did in previous sections. That is, correct F- 
statistics may now be constructed for testing the significance of treatment main effects and interactions. As well, the large number of observations due to replication will improve the estimates of $\sigma_{W P}^{2}$ and $\sigma_{S P}^{2}$ thus strengthening the power and precision in inference.

Having presented the general ANOVA model, we now consider the approach to analyzing the data. We will do this via a three-step process, where each of the three steps corresponds to one portion of the ANOVA model in Table 6.10. The steps are as follows:

1. To analyze the "block" portion of the BFFSP design, we view the $r 2^{b_{1}}$ blocks as the experimental units. The experiment as a whole is seen as a RCBD, with $2^{b_{1}}$ treatments in each of the replicates. Note that since the blocks are the experimental units, we perform the ANOVA on the block averages. (That is, average over the WP and SP treatment combinations within a given block to obtain one observation for the RCBD.)

2. The WP portion is analyzed as a BFF with $r 2^{b_{i}}$ blocks, an ANOVA being run on the WP averages. (That is, average over the SP treatment combinations associated with each WP treatment combination.) The $S S_{\text {Tot }}$ from step 1 will now be thought of as $S S_{B l o c k s}$ in the WP analysis.

3. The SP portion is also viewed as a $\mathrm{BFF}$, this time possessing $r 2^{n_{1}-k_{1}}$ blocks, where the blocks are the WPs. The $S S_{\text {Tot }}$ from step 2 will be viewed as $S S_{B l o c k s}$ in the SP analysis.

\subsubsection{The ANOVA Model for Blocking via Separation: With Replication}

A replicated $2^{\left(n_{1}+n_{2}\right)-\left(k_{1}+k_{2}\right) \pm\left(0+b_{2}\right)}$ BFFSP design has $r 2^{\left(n_{1}+n_{2}\right)-\left(k_{1}+k_{2}\right)}$ treatment combinations to be run. Again, we must account for the resulting $r 2^{\left(n_{1}+n_{2}\right)-\left(k_{1}+k_{2}\right)}-1 \mathrm{df}$ in the corresponding ANOVA model (see Table 6.11).

Similar to the BFFSP design with pure WP blocking, we have $2^{b_{2}}$ blocks per replicate for a total of $r 2^{b_{2}}$ blocks in the design. This implies that $r 2^{b_{2}}-1 \mathrm{df}$ will be reserved for the block portion of the design. The other df may be accounted for 
Table 6.11: ANOVA Model for a Replicated $2^{\left(n_{1}+n_{2}\right)-\left(k_{1}+k_{2}\right) \pm\left(0+b_{2}\right)}$ BFFSP Design.

\begin{tabular}{lll}
\hline \hline \multicolumn{1}{c}{ Source of Variation } & \multicolumn{1}{c}{ Degrees of Freedom } \\
\hline Blocks & & \\
& Replicates & $r-1$ \\
& Separators & $2^{b_{2}}-1$ \\
& Error & $(r-1)\left(2^{b_{2}}-1\right)$ \\
& & $r 2^{b_{2}}-1$ \\
\hline Block Total & & \\
\hline WP & Effects & $2^{n_{1}-k_{1}+b_{2}}-2^{b_{2}}$ \\
& Error & $(r-1)\left(2^{n_{1}-k_{1}+b_{2}}-2^{b_{2}}\right)$ \\
\hline WP Total & & $r\left(2^{n_{1}-k_{1}+b_{2}}-2^{b_{2}}\right)$ \\
\hline SP & & \\
& Effects & $2^{\left(n_{1}+n_{2}\right)-\left(k_{1}+k_{2}\right)}-2^{n_{1}-k_{1}+b_{2}}$ \\
& Error & $(r-1)\left(2^{\left(n_{1}+n_{2}\right)-\left(k_{1}+k_{2}\right)}-2^{n_{1}-k_{1}+b_{2}}\right)$ \\
\hline \hline Design Total & & $r 2^{\left(n_{1}+n_{2}\right)-\left(k_{1}+k_{2}\right)}-1$ \\
\hline \hline
\end{tabular}

by considering the discussion in Section 6.4.3 regarding unreplicated BFFSP designs obtained via separation.

Again, as a result of the design replicates we may calculate $M S E_{W P}$ and $M S E_{S P}$ as estimates of $\sigma_{W P}^{2}$ and $\sigma_{S P}^{2}$, respectively. F-statistics may then be constructed for testing the significance of treatment main effects and interactions. Tests for significance of block effects may also be performed by use of the block error estimate, replicates $\times$ separators.

As in Section 6.5.1, we now present the three-step approach to the analysis of a $2^{\left(n_{1}+n_{2}\right)-\left(k_{1}+k_{2}\right) \pm\left(0+b_{2}\right)}$ BFFSP design:

1. To analyze the "block" portion of the BFFSP design, we view the $r 2^{b_{2}}$ blocks as the experimental units. The experiment as a whole is seen as a RCBD, with $2^{b_{2}}$ treatments in each of the replicates. Note that since the blocks are the experimental units, we perform the ANOVA on the block averages. (That is, average over the WP and SP treatment combinations within a given block to 
obtain one observation for the RCBD.)

2. The WP portion is analyzed as a BFF with $r 2^{b_{2}}$ blocks, an ANOVA being run on the WP averages. (That is, average over the SP treatment combinations associated with each WP treatment combination.) The $S S_{T o t}$ from step 1 will now be thought of as $S S_{\text {Blocks }}$ in the WP analysis.

3. The SP portion is also viewed as a BFF, this time possessing $r 2^{n_{1}-k_{1}+b_{2}}$ blocks, where the blocks are the WPs. The $S S_{\text {Tot }}$ from step 2 will be viewed as $S S_{\text {Blocks }}$ in the SP analysis.

\subsubsection{The ANOVA Model for Mixed Blocking: With Repli- cation}

The ANOVA model for a $2^{\left(n_{1}+n_{2}\right)-\left(k_{1}+k_{2}\right) \pm\left(b_{1}+b_{2}\right)}$ BFFSP design with mixed blocking is an amalgamation of Tables 6.10 and 6.11. Any BFFSP ANOVA model discussed thus far can be derived from the general model for mixed blocking (see Table 6.12).

Here we have $2^{b_{1}+b_{2}}$ blocks per replicate for a total of $r 2^{b_{1}+b_{2}}$ blocks in the design; therefore, $r 2^{b_{1}+b_{2}}-1 \mathrm{df}$ will be reserved for the block portion of the design. Also, observe that $r\left(2^{n_{1}-k_{1}+b_{2}}-2^{b_{1}+b_{2}}\right)$ df are available for the WP portion in Table 6.12. The remaining design $\mathrm{df}$ are distributed among the SP effects and SP error.

The three-step approach to analysis is easily extended to $2^{\left(n_{1}+n_{2}\right)-\left(k_{1}+k_{2}\right) \pm\left(b_{1}+b_{2}\right)}$ BFFSP designs:

1. To analyze the "block" portion of the BFFSP design, we view the $r 2^{b_{1}+b_{2}}$ blocks as the experimental units. The experiment as a whole is seen as a RCBD, with $2^{b_{1}+b_{2}}$ treatments in each of the replicates. Note that since the blocks are the experimental units, we perform the ANOVA on the block averages. (That is, average over the WP and SP treatment combinations within a given block to obtain one observation for the RCBD.)

2. The WP portion is analyzed as a BFF with $r 2^{b_{1}+b_{2}}$ blocks, an ANOVA being run on the WP averages. (That is, average over the SP treatment combinations associated with each WP treatment combination.) The $S S_{T o t}$ from step 1 will now be thought of as $S S_{\text {Blocks }}$ in the WP analysis. 
Table 6.12: ANOVA Model for a Replicated $2^{\left(n_{1}+n_{2}\right)-\left(k_{1}+k_{2}\right) \pm\left(b_{1}+b_{2}\right)}$ BFFSP Design.

\begin{tabular}{lll}
\hline \hline \multicolumn{1}{c}{ Source of Variation } & \multicolumn{1}{c}{ Degrees of Freedom } \\
\hline Blocks & & $r-1$ \\
& Replicates & $2^{b_{1}}-1$ \\
& Pure WP Blocks & $2^{b_{2}}-1$ \\
& Separators & $\left.2^{b_{1}}-1\right)\left(2^{b_{2}}-1\right)$ \\
& Pure WP Blocks $\times$ Sep's & $(r-1)\left(2^{b_{1}+b_{2}}-1\right)$ \\
& Error & $r 2^{b_{1}+b_{2}}-1$ \\
\hline Block Total & & \\
\hline WP & & $2^{n_{1}-k_{1}+b_{2}}-2^{b_{1}+b_{2}}$ \\
& Effects & $(r-1)\left(2^{n_{1}-k_{1}+b_{2}}-2^{b_{1}+b_{2}}\right)$ \\
& Error & $r\left(2^{n_{1}-k_{1}+b_{2}}-2^{b_{1}+b_{2}}\right)$ \\
\hline WP Total & & \\
\hline SP & & $2^{\left(n_{1}+n_{2}\right)-\left(k_{1}+k_{2}\right)}-2^{n_{1}-k_{1}+b_{2}}$ \\
& Effects & $(r-1)\left(2^{\left(n_{1}+n_{2}\right)-\left(k_{1}+k_{2}\right)}-2^{n_{1}-k_{1}+b_{2}}\right)$ \\
\hline \hline Design Total & & $r 2^{\left(n_{1}+n_{2}\right)-\left(k_{1}+k_{2}\right)}-1$ \\
\hline \hline
\end{tabular}

3. The SP portion is also viewed as a BFF, this time possessing $r 2^{n_{1}-k_{1}+b_{2}}$ blocks, where the blocks are the WPs. The $S S_{\text {Tot }}$ from step 2 will be viewed as $S S_{\text {Blocks }}$ in the SP analysis. 


\section{Chapter 7}

\section{Results Analogous to Those for FFSP Designs}

Chen and $\mathrm{Wu}$ (1991) provided an approach to summarizing the DCSs of FF designs through the use of matrices. Subsequently, Bingham and Sitter (1999b) extended this matrix representation to the FFSP design scenario. These matrix representations were then utilized in the development of theoretical results for two-level FF and FFSP designs with emphasis on the resolution and MA criteria. In this chapter we develop extensions to the matrix representation of FFSP designs by allowing for the presence of blocking variables in the derivations. Notation is developed first through an example while theoretical results are established by more detailed discussions in subsequent sections.

\subsection{Matrix Representation of BFFSP Designs: No- tational Development}

Example 7.1.1 Suppose an experimenter intends to run a $2^{(5+3)-(1+1) \pm(1+1)}$ BFFSP design. One possible design has factor and blocking generators given by $E=A B C, \beta_{1}=$ $B C D, r=A p q$ and $\delta_{1}=B p q$. The resulting DCS for this design is $I=A B C E=$ $B C D \beta_{1}=A p q r=B p q \delta_{1}=A D E \beta_{1}=B C E p q r=A C E p q \delta_{1}=A B C D p q r \beta_{1}=$ $C D p q \beta_{1} \delta_{1}=A B r \delta_{1}=D E p q r \beta_{1}=A B D E p q \beta_{1} \delta_{1}=C E r \delta_{1}=A C D r \beta_{1} \delta_{1}=$ $B D E r \beta_{1} \delta_{1}$. The matrix form of the DCS for the $2^{(5+3)-(1+1) \pm(1+1)}$ BFFSP design is given in Table 7.1. As in Bingham and Sitter (1999b), $w_{i}$ denotes the $i^{\text {th }}$ word in 
the design's DCS. If a factor or blocking variable is present within $w_{i}$ it is identified with a 1 in its corresponding column.

By looking closely at Table 7.1 we see that columns " $D$ " and " $\beta_{1}$ " are identical. Also, columns " $p$ " and " $q$ " are identical. Instead of writing down these redundant columns one may represent a BFFSP design by providing the matrix containing only the unique columns from the design's DCS matrix as well as a frequency vector, $f$.

Table 7.1: Matrix Representation of the DCS for the $2^{(5+3)-(1+1) \pm(1+1)}$ Design

\begin{tabular}{l||cccccccccc}
\hline \hline & $\mathrm{A}$ & $\mathrm{B}$ & $\mathrm{C}$ & $\mathrm{D}$ & $\mathrm{E}$ & $\beta_{1}$ & $\mathrm{p}$ & $\mathrm{q}$ & $\mathrm{r}$ & $\delta_{1}$ \\
\hline$w_{1}$ & 1 & 1 & 1 & 0 & 1 & 0 & 0 & 0 & 0 & 0 \\
$w_{2}$ & 0 & 1 & 1 & 1 & 0 & 1 & 0 & 0 & 0 & 0 \\
$w_{3}$ & 1 & 0 & 0 & 0 & 0 & 0 & 1 & 1 & 1 & 0 \\
$w_{4}$ & 0 & 1 & 0 & 0 & 0 & 0 & 1 & 1 & 0 & 1 \\
$w_{5}$ & 1 & 0 & 0 & 1 & 1 & 1 & 0 & 0 & 0 & 0 \\
$w_{6}$ & 0 & 1 & 1 & 0 & 1 & 0 & 1 & 1 & 1 & 0 \\
$w_{7}$ & 1 & 0 & 1 & 0 & 1 & 0 & 1 & 1 & 0 & 1 \\
$w_{8}$ & 1 & 1 & 1 & 1 & 0 & 1 & 1 & 1 & 1 & 0 \\
$w_{9}$ & 0 & 0 & 1 & 1 & 0 & 1 & 1 & 1 & 0 & 1 \\
$w_{10}$ & 1 & 1 & 0 & 0 & 0 & 0 & 0 & 0 & 1 & 1 \\
$w_{11}$ & 0 & 0 & 0 & 1 & 1 & 1 & 1 & 1 & 1 & 0 \\
$w_{12}$ & 1 & 1 & 0 & 1 & 1 & 1 & 1 & 1 & 0 & 1 \\
$w_{13}$ & 0 & 0 & 1 & 0 & 1 & 0 & 0 & 0 & 1 & 1 \\
$w_{14}$ & 1 & 0 & 1 & 1 & 0 & 1 & 0 & 0 & 1 & 1 \\
$w_{15}$ & 0 & 1 & 0 & 1 & 1 & 1 & 0 & 0 & 1 & 1 \\
\hline
\end{tabular}

Consequently, the $2^{(5+3)-(1+1) \pm(1+1)}$ design may be summarized by the reduced matrix, 


$$
\mathbf{M}_{1}=\left(\begin{array}{llllllll}
1 & 1 & 1 & 0 & 1 & 0 & 0 & 0 \\
0 & 1 & 1 & 1 & 0 & 0 & 0 & 0 \\
1 & 0 & 0 & 0 & 0 & 1 & 1 & 0 \\
0 & 1 & 0 & 0 & 0 & 1 & 0 & 1 \\
1 & 0 & 0 & 1 & 1 & 0 & 0 & 0 \\
0 & 1 & 1 & 0 & 1 & 1 & 1 & 0 \\
1 & 0 & 1 & 0 & 1 & 1 & 0 & 1 \\
1 & 1 & 1 & 1 & 0 & 1 & 1 & 0 \\
0 & 0 & 1 & 1 & 0 & 1 & 0 & 1 \\
1 & 1 & 0 & 0 & 0 & 0 & 1 & 1 \\
0 & 0 & 0 & 1 & 1 & 1 & 1 & 0 \\
1 & 1 & 0 & 1 & 1 & 1 & 0 & 1 \\
0 & 0 & 1 & 0 & 1 & 0 & 1 & 1 \\
1 & 0 & 1 & 1 & 0 & 0 & 1 & 1 \\
0 & 1 & 0 & 1 & 1 & 0 & 1 & 1
\end{array}\right)
$$

and the frequency vector $f=(1,1,1,2,1,2,1,1)$, where $f_{i}$ is the number of factors associated with the $i^{\text {th }}$ column of $\mathbf{M}_{\mathbf{1}}$.

We may identify which factors are WP factors, pure WP blocking variables, SP factors or separators by introducing an extension of the (split-plot) frequency matrix first described by Chen and $W u$ (1991). For the $2^{(5+3)-(1+1) \pm(1+1)}$ design in this example,

$$
\boldsymbol{f}=\left(\begin{array}{llllllll}
1 & 1 & 1 & 1 & 1 & 0 & 0 & 0 \\
0 & 0 & 0 & 1 & 0 & 0 & 0 & 0 \\
0 & 0 & 0 & 0 & 0 & 2 & 1 & 0 \\
0 & 0 & 0 & 0 & 0 & 0 & 0 & 1
\end{array}\right)
$$

In our representation, $\left(f_{1, i}, f_{2, i}, f_{3, i}, f_{4, i}\right)^{\prime}$ implies that column $i$ of $\mathbf{M}_{\mathbf{1}}$ occurs (or has a frequency of) $f_{1, i}+f_{2, i}+f_{3, i}+f_{4, i}$ times. Of this number, $f_{1, i}$ is due to WP factors, $f_{2, i}$ is due to pure WP blocking variables, $f_{3, i}$ is due to SP factors and $f_{4, i}$ is due to separators. It can be shown that by using $\mathbf{M}_{\mathbf{1}}$ and $f$ one could work "backwards" and reconstruct Table 7.1, the DCS of the $2^{(5+3)-(1+1) \pm(1+1)}$ BFFSP design. 


\subsection{Development of a General Matrix Represen- tation for BFFSP Designs}

The objective of this section is to extend the general matrix framework found in the literature (developed for the FF and FFSP settings) to that of the BFFSP design scenario. Consequently, the results that follow in this and subsequent sections closely parallel those found in Chen and Wu (1991) and Bingham and Sitter (1999b). In this particular section we intend to simply adjust the existing general FFSP design matrix representation by allowing for the inclusion of blocking variables.

Let $\mathbf{M}_{2}$ be a $\left(2^{d}-1\right) \times\left(2^{d}-1\right)$ matrix where

$$
\mathbf{M}_{2}=\left(\begin{array}{cc}
I_{d} & B \\
B^{\prime} & B^{\prime} B
\end{array}\right),
$$

and $d=k_{1}+k_{2}+b_{1}+b_{2} . I_{d}$ denotes the $d \times d$ identity matrix while the set of columns of $\left(I_{d}, B\right)$ form the vector space spanned by the columns of $I_{d}$ over the finite field $G F(2)$. Likewise, the rows of $\mathbf{M}_{2}$ form the vector space spanned by the rows of $\left(I_{d}, B\right)$ over the finite field $G F(2)$. Note that the identity column and row of 0 's has been excluded from $\mathbf{M}_{2}$ thereby reducing its dimensions from what otherwise would have been $2^{d} \times 2^{d}$. If we replace the 0 's and 1's in $\mathbf{M}_{2}$ with 1's and -1's, respectively, and add a column and row of 1's, $\mathbf{M}_{2}$ would be equivalent to a $2^{d} \times 2^{d}$ Hadamard matrix, $H_{2^{d}}$. This equivalence with Hadamard matrices will provide us with several useful properties for proving theorems in following sections. (For a brief introduction to finite fields and Hadamard matrices, the reader is encouraged to read the relevant sections of Durbin (2000) and Hedayat and Wallis (1978), respectively.) Note that $\mathbf{M}_{\mathbf{1}}$, in Section 7.1, is in a slightly different form than $\mathbf{M}_{\mathbf{2}}$ in that it is a subset of columns from $\mathbf{M}_{2}$, with a permutation of the columns and rows of $\mathbf{M}_{2}$. These differences between $\mathbf{M}_{\mathbf{1}}$ and $\mathbf{M}_{\mathbf{2}}$ do not affect the inherent structure of the corresponding BFFSP design.

When constructing a BFFSP design we must specify which factors are WP factors, pure WP blocking variables, SP factors and separators. To assign these factors to the columns of $\mathbf{M}_{2}$, we may begin by partitioning the first $d$ rows of $\mathbf{M}_{2}$ into the following form,

$$
\left(\begin{array}{ccccc}
I_{k_{1}+b_{1}} & 0 & B_{1} & 0 & C_{1} \\
0 & I_{k_{2}+b_{2}} & 0 & B_{2} & C_{2}
\end{array}\right) .
$$


$I_{k_{1}+b_{1}}$ and $I_{k_{2}+b_{2}}$ represent the $\left(k_{1}+b_{1}\right) \times\left(k_{1}+b_{1}\right)$ and $\left(k_{2}+b_{2}\right) \times\left(k_{2}+b_{2}\right)$ identity matrices, respectively. Similar to that for $\left(I_{d}, B\right)$ in 7.1, the columns of $\left(I_{k_{1}+b_{1}}, B_{1}\right)$ and $\left(I_{k_{2}+b_{2}}, B_{2}\right)$ form the subspace spanned by the columns of $I_{k_{1}+b_{1}}$ and $I_{k_{2}+b_{2}}$ respectively, excluding the identity columns of 1 's. This implies that $B_{1}$ will have $2^{k_{1}+b_{1}}-\left(k_{1}+b_{1}\right)-1$ columns; likewise, $B_{2}$ will contain $2^{k_{2}+b_{2}}-\left(k_{2}+b_{2}\right)-1$ columns. The remaining $2^{k_{1}+k_{2}+b_{1}+b_{2}}-2^{k_{1}+b_{1}}-2^{k_{2}+b_{2}}+1$ columns in $\left(C_{1}, C_{2}\right)^{\prime}$ form a subset of the vector space spanned jointly by the columns of

$$
\mathbf{I}_{\mathbf{d}}=\left(\begin{array}{cc}
I_{k_{1}+b_{1}} & 0 \\
0 & I_{k_{2}+b_{2}}
\end{array}\right) .
$$

The complete, "expanded" matrix form for $\mathbf{M}_{2}$ is given by

$$
\mathbf{M}_{2}=\left(\begin{array}{ccccc}
I_{k_{1}+b_{1}} & 0 & B_{1} & 0 & C_{1} \\
0 & I_{k_{2}+b_{2}} & 0 & B_{2} & C_{2} \\
B_{1}^{\prime} & 0 & B_{1}^{\prime} B_{1} & 0 & B_{1}^{\prime} C_{1} \\
0 & B_{2}^{\prime} & 0 & B_{2}^{\prime} B_{2} & B_{2}^{\prime} C_{2} \\
C_{1}^{\prime} & C_{2}^{\prime} & C_{1}^{\prime} B_{1} & C_{2}^{\prime} B_{2} & C_{1}^{\prime} C_{1}+C_{2}^{\prime} C_{2}
\end{array}\right),
$$

where $\mathbf{B}$, from 7.1 , is,

$$
\left(\begin{array}{ccc}
B_{1} & 0 & C_{1} \\
0 & B_{2} & C_{2}
\end{array}\right)
$$

such that,

$$
\mathbf{B}^{\prime} \mathbf{B}=\left(\begin{array}{ccc}
B_{1}^{\prime} B_{1} & 0 & B_{1}^{\prime} C_{1} \\
0 & B_{2}^{\prime} B_{2} & B_{2}^{\prime} C_{2} \\
C_{1}^{\prime} B_{1} & C_{2}^{\prime} B_{2} & C_{1}^{\prime} C_{1}+C_{2}^{\prime} C_{2}
\end{array}\right)
$$

in $\mathbf{M}_{\mathbf{2}}$.

Reordering the columns of $\mathbf{M}_{\mathbf{2}}$ in the style of Bingham and Sitter (1999b), we obtain $\mathbf{M}=\left(A_{1}, A_{2}\right)$, where,

$$
\mathbf{A}_{1}=\left(\begin{array}{cc}
0 & 0 \\
I_{k_{2}+b_{2}} & B_{2} \\
0 & 0 \\
B_{2}^{\prime} & B_{2}^{\prime} B_{2} \\
C_{2}^{\prime} & C_{2}^{\prime} B_{2}
\end{array}\right)
$$


and

$$
\mathbf{A}_{2}=\left(\begin{array}{ccc}
I_{k_{1}+b_{1}} & B_{1} & C_{1} \\
0 & 0 & C_{2} \\
B_{1}^{\prime} & B_{1}^{\prime} B_{1} & B_{1}^{\prime} C_{1} \\
0 & 0 & B_{2}^{\prime} C_{2} \\
C_{1}^{\prime} & C_{1}^{\prime} B_{1} & C_{1}^{\prime} C_{1}+C_{2}^{\prime} C_{2}
\end{array}\right)
$$

A BFFSP design is now able to be fully represented by the matrix $\mathbf{M}$ and its corresponding split-plot frequency matrix,

$$
\mathbf{f}=\left(\begin{array}{ccccccc}
f_{1,1}, & f_{1,2}, & \ldots, & f_{1,2^{k_{2}+b_{2}}-1}, & f_{1,2^{k_{2}+b_{2}}}, & \ldots, & f_{1,2^{d}-1} \\
f_{2,1}, & f_{2,2}, & \ldots, & f_{2,2^{k_{2}+b_{2}}-1}, & f_{2,2^{k_{2}+b_{2}}}, & \ldots, & f_{2,2^{d}-1} \\
f_{3,1}, & f_{3,2}, & \ldots, & f_{3,2^{k_{2}+b_{2}}-1}, & 0, & \ldots, & 0 \\
f_{4,1}, & f_{4,2}, & \ldots, & f_{4,2^{k_{2}+b_{2}-1}}, & 0, & \ldots, & 0
\end{array}\right),
$$

where the frequencies in the $i^{\text {th }}$ column of $\mathbf{f}$ correspond to the number of factors and blocking variables assigned to the $i^{\text {th }}$ column of $\mathbf{M}$. Of course, we must have $\sum f_{1, i}=n_{1}, \sum f_{2, i}=b_{1}, \sum f_{3, i}=n_{2}$ and $\sum f_{4, i}=b_{2}$.

In Chapter 3 it was shown that SP generators may be comprised of both WP and SP factors. In Chapter 3 we also demonstrated that separators may contain both WP and SP factors. Because of this we may assign WP factors and WP blocking variables to any of the $2^{d}-1$ columns of $\mathbf{M}$. However, WP generators and WP blocking variables cannot contain SP factors. As a result, we may not assign the $n_{2}$ SP factors and $b_{2}$ separators to all of the columns of $\mathbf{M}$. Therefore, $f_{3, i}$ and $f_{4, i}$ may only be nonzero for columns in $A_{1}$. The rationalization for rearranging $\mathbf{M}_{\mathbf{2}}$ into the form $\mathbf{M}=\left(A_{1}, A_{2}\right)$ is now more apparent. The usefulness of the form of $\mathbf{M}$ will be even greater appreciated when theorems are derived in the following sections.

\subsection{Main Results}

Now that we have developed a general matrix representation for BFFSP designs we may formulate some useful theoretical results, keeping in mind the restrictions on the assignment of both SP factors and separators to the columns of $\mathrm{M}$. The yet-to-be established results will provide means by which we may construct larger 
BFFSP designs from other, smaller BFFSP designs. In addition, resolution and MA properties of these larger designs will be investigated.

The theorems presented in this chapter are analogous to the ones presented by Chen and Wu (1991) and Bingham and Sitter (1999b) with respect to the FF and FFSP settings, respectively. In those papers, theoretical results were also motivated by the corresponding general matrix representations for FF and FFSP designs. Because of this parallel, proofs will be developed in a fashion very similar to that of the preceding references. Adaptations to the proofs will primarily occur when accounting for the presence of blocking variables, the revised form of the split-plot frequency matrix, and the existence of fractional word-lengths in a BFFSP design's WLP.

\subsubsection{Adding Both WP and SP Factors to an Existing BFFSP Design}

Theorem 7.1 Let $D\left(n_{1}, n_{2} ; k_{1}, k_{2} ; b_{1}, b_{2}\right)$ be a $2^{\left(n_{1}+n_{2}\right)-\left(k_{1}+k_{2}\right) \pm\left(b_{1}+b_{2}\right)}$ BFFSP design with $W L P W$, and let $\operatorname{lag}(W, m)=(0,0, \ldots, 0, W)$ be the lag vector of the WLP $W$ with $m$ leading zeroes. For $0 \leq r \leq 2^{k_{2}+b_{2}}-1$ there exists a $D\left(n_{1}+2^{d}-r-1, n_{2}+\right.$ $\left.r ; k_{1}, k_{2} ; b_{1}, b_{2}\right)$ with WLP $\operatorname{lag}\left(W, 2^{d}\right)$, where $d=k_{1}+k_{2}+b_{1}+b_{2}$.

Proof : Let $D_{1}$ be a $D\left(n_{1}, n_{2} ; k_{1}, k_{2} ; b_{1}, b_{2}\right)$ BFFSP design represented by $(\mathbf{M}, \mathbf{f})$. Also, let $\mathbf{f}^{*}$ be a split-plot frequency matrix with $2^{d}-1$ columns $\left(f_{1, i}^{*}, f_{2, i}^{*}, f_{3, i}^{*}, f_{4, i}^{*}\right)^{\prime}$ such that there are $2^{d}-r-1$ columns in $\mathbf{f}^{*}$ of the form $(1,0,0,0)^{\prime}$ and $r$ columns in $\mathbf{f}^{*}$ of the form $(0,0,1,0)^{\prime}$. Columns of the form $(0,0,1,0)^{\prime}$ are assigned only to columns in $A_{1}$ while the $2^{d}-r-1$ columns of the form $(1,0,0,0)^{\prime}$ are assigned to the remaining columns of $\mathbf{M}$. Therefore, $\sum\left(f_{1, i}+f_{1, i}^{*}\right)=n_{1}+2^{d}-r-1$ and $\sum\left(f_{3, i}+f_{3, i}^{*}\right)=n_{2}+r$.

By letting $D_{2}$ be a BFFSP design represented by $\left(\mathbf{M}, \mathbf{f}+\mathbf{f}^{*}\right)$, we are in effect adding $r$ new SP factors and $2^{d}-r-1$ new WP factors to $D_{1}$. Clearly then, $D_{2}$ is a $D\left(n_{1}+2^{d}-r-1, n_{2}+r ; k_{1}, k_{2} ; b_{1}, b_{2}\right)$ BFFSP design. Since $\mathbf{M}$ is equivalent to a Hadamard matrix, excluding the identity column and row of 1's, by a property of such matrices we know that there are $2^{d-1} 1$ 's in each row of $\mathbf{M}$ (Hedayat, Sloane and Stufken, 1999, p.147). Now by assigning each of the $2^{d}-1$ new treatment factors to separate columns of $\mathbf{M}$, each word in the DCS of $D_{2}$ is $2^{d-1}$ longer than its corresponding word in $D_{1}$.

As first described in Section 3.2, let $W=\left(A_{3}, A_{3.5}, A_{4}, A_{4.5}, \ldots, A_{n_{1}+n_{2}+1.5}\right)$ rep- 
resent the WLP of a $2^{\left(n_{1}+n_{2}\right)-\left(k_{1}+k_{2}\right) \pm\left(b_{1}+b_{2}\right)}$ BFFSP design. For an increase of $2^{d-1}$ in the length of each word, we must then move each entry, $A_{i}, 2 * 2^{d-1}=2^{d}$ positions to the right in $W$ as a consequence of both the increased word-length and the presence of fractional resolution in the definition of $W$. We see that in comparison to $W$ for $D_{1}, D_{2}$ 's WLP must have $2^{d}$ leading zeros so that it becomes $\operatorname{lag}\left(W, 2^{d}\right)$.

Example 7.3.1 Consider a 16-run $2^{(2+3)-(0+1) \pm(1+1)}$ BFFSP design with generators $\beta_{1}=A B, r=A B q, \delta_{1}=B p q$ and $W L P W_{1}$. For this design, $W_{1}=(0,2,1,4)$. Using Theorem 7.1, we may add a total of $2^{3}-1=7$ treatment factors to the $2^{(2+3)-(0+1) \pm(1+1)}$ design, with the restriction that at most 3 of the additional factors are to be SP factors. Using $r=2$, we infer that there exists a $2^{(7+5)-(0+1) \pm(1+1)} B F F S P$ design with $W L P$, say $W_{2}$, such that $W_{2}=\operatorname{lag}\left(W_{1}, 2^{3}\right)=(0,0,0,0,0,0,0,0,0,2,1,4)$. The larger design requires that 2,048 treatment combinations be run; this design is, for all intents and purposes, too large to be considered for a real-life experiment. Later, we will show how one may construct larger, yet more "reasonably-sized" BFFSP designs.

Theorem 7.1 allows us to add a combination of WP and SP factors to an existing BFFSP design thereby providing an explicit method for constructing larger BFFSP designs from smaller ones. Theorem 7.1 also allows us to simultaneously predict the larger design's WLP. The restriction $\left(0 \leq r \leq 2^{k_{2}+b_{2}}-1\right)$ on the number of SP factors which we may add is a direct result of the fact that we must assign SP factors only to the $2^{k_{2}+b_{2}}-1$ columns of $A_{1}$. If we were to set $r=0$, we would be adding WP factors exclusively. Having $r=0$ would probably be of interest to an experimenter only if there are considerably more WP factors than SP factors comprising the process under investigation.

This approach to adding additional treatment factors to an existing BFFSP design is limited in that it allows only for the addition of basic (independent) treatment factors. Since blocking variables are always generated by other (WP or SP) factors this construction method does not allow for the inclusion of additional blocking variables in an existing BFFSP design. This observation also holds for other theorems provided in this section.

By the definition of a BFFSP design we must have that $k_{1}+k_{2} \geq 1$ and $b_{1}+b_{2} \geq 1$. Thus, by applying Theorem 7.1 we are adding a minimum of $2^{2}-1=3$ basic factors in forming the larger design. This implies that a larger design, constructed 
via Theorem 7.1, will always possess at least $2^{3}=8$ times as many runs as the smaller BFFSP design from which it was formed.

Since Theorem 7.1 provides us with the WLP of the larger design, we then also know that design's resolution. This knowledge could aid in the search for larger MA BFFSP designs. That is, having constructed a $2^{\left(n_{1}+2^{d}-r-1+n_{2}+r\right)-\left(k_{1}+k_{2}\right) \pm\left(b_{1}+b_{2}\right)}$ BFFSP design, say $D_{1}$, using Theorem 7.1, we have in effect obtained a lower bound for the resolution of the MA BFFSP design with equal $n_{1}, n_{2}, k_{1}, k_{2}, b_{1}$ and $b_{2}$ values. Therefore, we could compare $D_{1}$ 's resolution with the resolution of other BFFSP designs constructed perhaps via a computer algorithm. Designs with resolution less than that of $D_{1}$ could then be immediately discarded since we now know that the MA BFFSP design must have resolution at least that of $D_{1}$. This would assist in reducing search times for large BFFSP designs.

Having constructed a larger BFFSP design using Theorem 7.1, the following theorem allows us to place a lower bound on this design's maximum resolution.

Theorem 7.2 Let $R\left(n_{1}, n_{2} ; k_{1}, k_{2} ; b_{1}, b_{2}\right)$ be the maximum resolution for a $D\left(n_{1}, n_{2}\right.$; $\left.k_{1}, k_{2} ; b_{1}, b_{2}\right)$ BFFSP design. For $0 \leq r \leq 2^{k_{2}+b_{2}}-1, R\left(n_{1}+2^{d}-r-1, n_{2}+\right.$ $\left.r ; k_{1}, k_{2} ; b_{1}, b_{2}\right) \geq 2^{d-1}+R\left(n_{1}, n_{2} ; k_{1}, k_{2} ; b_{1}, b_{2}\right)$.

Proof : Let $D_{1}$ be a maximum resolution $D\left(n_{1}, n_{2} ; k_{1}, k_{2} ; b_{1}, b_{2}\right)$ BFFSP design represented by $(\mathbf{M}, \mathbf{f})$ with WLP $W$. Also, let $D_{2}$ be a BFFSP design corresponding to $\left(\mathbf{M}, \mathbf{f}+\mathbf{f}^{*}\right)$, ( $\mathbf{f}^{*}$ being defined in the proof of Theorem 7.1). By Theorem 7.1, $D_{2}$ is a $D\left(n_{1}+2^{d}-r-1, n_{2}+r ; k_{1}, k_{2} ; b_{1}, b_{2}\right)$ BFFSP design with WLP $\operatorname{lag}\left(W, 2^{d}\right)$ having resolution $R\left(n_{1}, n_{2} ; k_{1}, k_{2} ; b_{1}, b_{2}\right)+2^{d-1}$. Therefore, the maximum resolution $D\left(n_{1}+2^{d}-r-1, n_{2}+r ; k_{1}, k_{2} ; b_{1}, b_{2}\right)$ design must have resolution greater than or equal to $2^{d-1}+R\left(n_{1}, n_{2} ; k_{1}, k_{2} ; b_{1}, b_{2}\right)$.

Theorems 7.1 and 7.2 assist in the search for large MA BFFSP designs. It would be beneficial to have an explicit form for the upper bound of the resolution, but this is not possible. However, using methods very similar to Chen and Wu (1991) and Bingham and Sitter (1999b) we show that the maximum resolution of a BFFSP design is periodic. This result is summarized in the following theorem.

Theorem 7.3 For any fixed $n_{1}, n_{2}, k_{1}, k_{2}, b_{1}, b_{2}$ and $0 \leq r \leq 2^{k_{2}+b_{2}}-1$, there exists $L_{1}$ such that $R\left(n_{1}+l\left(2^{d}-r-1\right), n_{2}+l r ; k_{1}, k_{2} ; b_{1}, b_{2}\right)=2^{d-1}+R\left(n_{1}+(l-1)\left(2^{d}-\right.\right.$ 
$\left.r-1), n_{2}+(l-1) r ; k_{1}, k_{2} ; b_{1}, b_{2}\right) \forall l>L_{1}$. That is, there exists $L_{1}$ such that $\forall l>L_{1}$ the maximum resolution of a BFFSP design is periodic.

Proof: The proof is by contradiction. Assume that there are infinitely many $l_{1, i}$ such that

$$
\begin{aligned}
& R\left(n_{1}+l_{1, i}\left(2^{d}-r-1\right), n_{2}+l_{1, i} r ; k_{1}, k_{2} ; b_{1}, b_{2}\right) \\
& \quad \geq 2^{d-1}+R\left(n_{1}+\left(l_{1, i}-1\right)\left(2^{d}-r-1\right), n_{2}+\left(l_{1, i}-1\right) r ; k_{1}, k_{2} ; b_{1}, b_{2}\right)+1 .
\end{aligned}
$$

Assuming (7.5), we then have an increasing sequence of $l_{1}$ 's, say $\left\{l_{1, i}\right\}_{i=0}^{\infty}$, such that (7.5) is true. Now by Theorem 7.2 we have that,

$$
\begin{aligned}
& R\left(n_{1}+\left(l_{1, i}-1\right)\left(2^{d}-r-1\right), n_{2}+\left(l_{1, i}-1\right) r ; k_{1}, k_{2} ; b_{1}, b_{2}\right) \\
& \quad \geq 2^{d-1}+R\left(n_{1}+\left(l_{1, i}-2\right)\left(2^{d}-r-1\right), n_{2}+\left(l_{1, i}-2\right) r ; k_{1}, k_{2} ; b_{1}, b_{2}\right) .
\end{aligned}
$$

Substituting this result into (7.5), we obtain,

$$
\begin{aligned}
& R\left(n_{1}+l_{1, i}\left(2^{d}-r-1\right), n_{2}+l_{1, i} r ; k_{1}, k_{2} ; b_{1}, b_{2}\right) \\
& \quad \geq 2 * 2^{d-1}+R\left(n_{1}+\left(l_{1, i}-2\right)\left(2^{d}-r-1\right), n_{2}+\left(l_{1, i}-2\right) r ; k_{1}, k_{2} ; b_{1}, b_{2}\right)+1 .
\end{aligned}
$$

Using (7.5) and Theorem 7.2,

$$
\begin{aligned}
& R\left(n_{1}+l_{1, i}\left(2^{d}-r-1\right), n_{2}+l_{1, i} r ; k_{1}, k_{2} ; b_{1}, b_{2}\right) \\
& \quad \geq\left(l_{1, i}-l_{1, i-1}\right) 2^{d-1}+R\left(n_{1}+l_{1, i-1}\left(2^{d}-r-1\right), n_{2}+l_{1, i-1} r ; k_{1}, k_{2} ; b_{1}, b_{2}\right)+2 .
\end{aligned}
$$

Eventually,

$$
\begin{gathered}
R\left(n_{1}+l_{1, i}\left(2^{d}-r-1\right), n_{2}+l_{1, i} r ; k_{1}, k_{2} ; b_{1}, b_{2}\right) \\
\quad \geq l_{1, i} 2^{d-1}+R\left(n_{1}, n_{2} ; k_{1}, k_{2} ; b_{1}, b_{2}\right)+i .
\end{gathered}
$$

Plotkin (1960) showed that there is an upper bound on the maximum resolution for a $2^{n-k} \mathrm{FF}$ design. Specifically, if $R(n, k)$ is the maximum resolution for the $2^{n-k} \mathrm{FF}$ design then

$$
R(n, k) \leq \frac{2^{k-1}}{2^{k}-1} n
$$

Because of the restriction on the assignment of SP factors and separators to the columns of $A_{1}$ in $\mathbf{M}$ (Section 7.2), it is clear that $R\left(n_{1}, n_{2} ; k_{1}, k_{2} ; b_{1}, b_{2}\right) \leq R(n, k)$. So, we have

$$
R\left(n_{1}, n_{2} ; k_{1}, k_{2} ; b_{1}, b_{2}\right) \leq \frac{2^{d-1}}{2^{d}-1}\left(n_{1}+n_{2}\right)
$$


for $d=k_{1}+k_{2}+b_{1}+b_{2}$. It follows from (7.7) that

$$
\begin{aligned}
& R\left(n_{1}+l_{1, i}\left(2^{d}-r-1\right), n_{2}+l_{1, i} r ; k_{1}, k_{2} ; b_{1}, b_{2}\right) \\
& \leq \frac{2^{d-1}}{2^{d}-1}\left(n_{1}+l_{1, i}\left(2^{d}-r-1\right)+n_{2}+l_{1, i} r\right) \\
&= \frac{2^{d-1}}{2^{d}-1}\left(n_{1}+l_{1, i}\left(2^{d}-1\right)+n_{2}\right) \\
&=\frac{2^{d-1}}{2^{d}-1}\left(n_{1}+n_{2}\right)+l_{1, i} 2^{d-1} .
\end{aligned}
$$

From (7.8)

$$
l_{1, i} 2^{d-1} \geq R\left(n_{1}+l_{1, i}\left(2^{d}-r-1\right), n_{2}+l_{1, i} r ; k_{1}, k_{2} ; b_{1}, b_{2}\right)-\frac{2^{d-1}}{2^{d}-1}\left(n_{1}+n_{2}\right)
$$

and from (7.6)

$$
\begin{aligned}
& l_{1, i} i^{d-1} \\
& \quad \leq R\left(n_{1}+l_{1, i}\left(2^{d}-r-1\right), n_{2}+l_{1, i} r ; k_{1}, k_{2} ; b_{1}, b_{2}\right)-R\left(n_{1}, n_{2} ; k_{1}, k_{2} ; b_{1}, b_{2}\right)-i .10
\end{aligned}
$$

Therefore, using (7.9) and (7.10) and multiplying through by -1 , we obtain,

$$
\frac{2^{d-1}}{2^{d}-1}\left(n_{1}+n_{2}\right) \geq R\left(n_{1}, n_{2} ; k_{1}, k_{2} ; b_{1}, b_{2}\right)+i .
$$

This is not true for $i \rightarrow \infty$ thereby establishing the contradiction.

In the FFSP setting both the maximum resolution and MA criteria are periodic. In Theorem 7.3 we showed that the maximum resolution of a BFFSP design is periodic. In the following theorem, we establish periodicity of the MA criterion for BFFSP designs.

Theorem 7.4 For any fixed $n_{1}, n_{2}, k_{1}, k_{2}, b_{1}, b_{2}$ and $0 \leq r \leq 2^{k_{2}+b_{2}}-1$, there exists $Q_{1}$, such that $\forall q>Q_{1}$, if the $M A 2^{\left\{\left[n_{1}+(q-1)\left(2^{d}-r-1\right)\right]+\left(n_{2}+(q-1) r\right)\right\}-\left(k_{1}+k_{2}\right) \pm\left(b_{1}+b_{2}\right)}$ BFFSP design has WLPW, then the MA $2^{\left\{\left[n_{1}+q\left(2^{d}-r-1\right)\right]+\left(n_{2}+q r\right)\right\}-\left(k_{1}+k_{2}\right) \pm\left(b_{1}+b_{2}\right)}$ BFFSP design has WLP lag $\left(W, 2^{d}\right)$.

Proof : Let $v_{q_{1, i}}$ be the number of shortest length words in the DCS of the MA $2^{\left\{\left[n_{1}+q_{1, i}\left(2^{d}-r-1\right)\right]+\left(n_{2}+q_{1, i} r\right)\right\}-\left(k_{1}+k_{2}\right) \pm\left(b_{1}+b_{2}\right)}$ BFFSP design, with $q_{1, i}<q_{1, j}, \forall i<j$. Using Theorems $7.1-7.3$ and the definition of MA for BFFSP designs (Section 3.2), 
$v_{q_{1, i}}$ has the property, $v_{q_{1, i}} \geq v_{q_{1, j}}$ for $q_{1, i} \leq q_{1, j}$. Therefore, there will exist a positive integer $v_{1}$, such that $v_{q_{1, i}}=v_{1}$ for sufficiently large $i$. By the periodicity of maximum resolution (Theorem 7.3) the limit to the number of words of shortest length is nonzero. Now recall that the MA criterion sequentially minimizes successively larger words in a design's WLP. This fact in conjunction with adaptations of Theorems 7.1 - 7.3 would enable us to similarly construct sequences for the number of words with the second shortest word-length, the third shortest word-length and so on, with each of these sequences having a nonegative integer as a limit. Note that if we have only finitely many sequences there will be a finite $Q_{1}>L_{1}$ ( $L_{1}$ from Theorem 7.3) such that for $q \geq Q_{1}, W_{q_{1, i}}=\operatorname{lag}\left(W, 2^{d} q_{1, i}\right)$, where $W_{q_{1, i}}$ and $W$ are the WLPs of MA $2^{\left\{\left[n_{1}+q_{1, i}\left(2^{d}-r-1\right)\right]+\left(n_{2}+q_{1, i} r\right)\right\}-\left(k_{1}+k_{2}\right) \pm\left(b_{1}+b_{2}\right)}$ and $2^{\left(n_{1}+n_{2}\right)-\left(k_{1}+k_{2}\right) \pm\left(b_{1}+b_{2}\right)}$ designs, respectively. The final justification for the $2^{d}$ leading zeroes in $W_{q_{1, i}}$ follows an argument identical to that in Theorem 7.1.

What we must now establish is that there are only finitely many sequences that need to be constructed for a MA $2^{\left\{\left[n_{1}+q_{1, i}\left(2^{d}-r-1\right)\right]+\left(n_{2}+q_{1, i} r\right)\right\}-\left(k_{1}+k_{2}\right) \pm\left(b_{1}+b_{2}\right)}$ BFFSP design. To show that there are finitely many sequences we need to show that the word-lengths lie in an interval of finite length for any $q_{1, i}$. Trivially, we know that the length of the shortest word in the DCS of any $2^{\left(n_{1}+n_{2}\right)-\left(k_{1}+k_{2}\right) \pm\left(b_{1}+b_{2}\right)}$ BFFSP design must be bounded below by 1 . In using Theorem 7.4 we will add $q_{1, i}\left(2^{d}-1\right)$ treatment factors to the design. Therefore, the length of the shortest word must be bounded below by $1+q_{1, i}\left(2^{d-1}\right)$.

To establish an upper bound on the length of the longest word in the DCS of a MA BFFSP design we begin by using an identity due to Brownlee, Kelly and Loraine (1948) and adapt it to include the presence of WP factors, SP factors and blocking variables. In the BFFSP setting this identity is,

$$
\sum i A_{i}=\left[n_{1}+q_{1, i}\left(2^{d}-r-1\right)+n_{2}+q_{1, i} r\right] 2^{d-1},
$$

which gives the sum of all the word-lengths in the DCS of a design. So, if the BFFSP design has resolution $R$, then given that there are $2^{d}-1$ words in the designs's DCS, the longest possible word-length is

$$
U \leq\left[n_{1}+q_{1, i}\left(2^{d}-r-1\right)+n_{2}+q_{1, i} r\right] 2^{d-1}-\left(2^{d}-2\right) R .
$$

Using Theorem 7.2, a MA $2^{\left\{\left[n_{1}+q_{1, i}\left(2^{d}-r-1\right)\right]+\left(n_{2}+q_{1, i} r\right)\right\}-\left(k_{1}+k_{2}\right) \pm\left(b_{1}+b_{2}\right)}$ design has the 
following lower bound for its shortest word-length,

$$
\begin{aligned}
& R\left(n_{1}+q_{1, i}\left(2^{d}-r-1\right), n_{2}+q_{1, i} r ; k_{1}, k_{2} ; b_{1}, b_{2}\right) \\
& \quad \geq q_{1, i} 2^{d-1}+R\left(n_{1}, n_{2} ; k_{1}, k_{2} ; b_{1}, b_{2}\right) .
\end{aligned}
$$

Let $R_{0}$ be the maximum resolution for the design with $q_{1, i}=0$. Then from (7.12) and (7.13), the longest word-length is bounded above by,

$$
U \leq\left[n_{1}+q_{1, i}\left(2^{d}-r-1\right)+n_{2}+q_{1, i} r\right] 2^{d-1}-\left(2^{d}-2\right)\left(q_{1, i} 2^{d-1}+R_{0}\right) .
$$

This simplifies to,

$$
U \leq\left(n_{1}+n_{2}\right) 2^{d-1}+q_{1, i} 2^{d-1}-R_{0}\left(2^{d}-2\right) .
$$

Hence, the word-lengths of a MA $2^{\left\{\left[n_{1}+q_{1, i}\left(2^{d}-r-1\right)\right]+\left(n_{2}+q_{1, i} r\right)\right\}-\left(k_{1}+k_{2}\right) \pm\left(b_{1}+b_{2}\right)}$ design must be in the range,

$$
\left[1+q_{1,2} 2^{d-1},\left(n_{1}+n_{2}\right) 2^{d-1}+q_{1,2} 2^{d-1}-R_{0}\left(2^{d}-2\right)\right] .
$$

The length of this interval is independent of $q_{1, i}$ and therefore of finite length. This proves the theorem.

Theorems 7.1 - 7.4 allow for the possibility of generating large MA BFFSP designs from smaller ones. However, MA designs constructed via these theorems will often be too large for all practical purposes since we are required to add $2^{d}-1$ basic factors to the smaller BFFSP designs. Recall that the addition of $2^{d}-1$ basic factors implies that the larger design will always possess at least $2^{3}=8$ times as many runs as does the smaller BFFSP design from which it was formed (Section 7.3.1).

In the next section we will consider adding only basic SP factors to existing $2^{\left(n_{1}+n_{2}\right)-\left(k_{1}+k_{2}\right) \pm\left(b_{1}+b_{2}\right)}$ designs. We will see that this provides an experimenter with "not-as-large" BFFSP designs. In addition, since SP factors are typically of more interest to the researcher from an inferential perspective, focusing exclusively on the addition of SP factors is intuitive.

\subsubsection{Adding Only SP Factors to an Existing BFFSP Design}

In Section 7.2 we demonstrated that one cannot add SP factors to each column of $\mathbf{M}$, whereas with WP factors one may. In doing so, we showed that one can add only 
$0 \leq r \leq 2^{k_{2}+b_{2}}-1$ basic SP factors to an existing BFFSP design whereas up to $2^{d}-1$ basic WP factors may be added. We have previously mentioned that this could result in the construction of very large BFFSP designs (Example 7.3.1 provided evidence of this).

What may be of more interest to the experimenter is a method that accommodates only the exclusive addition of SP treatment factors. We can guarantee the existence of larger BFFSP designs by adding SP factors to all of the $2^{k_{2}+b_{2}}-1$ columns of $A_{1}$ in $\mathrm{M}$, without adding any additional WP factors. In this setting the word-lengths of words in the DCS containing only WP factors and WP blocking variables will remain unchanged; changes in word-length will occur only in those words containing SP factors and separators.

The theorems presented in this section will be analogous to Theorems 7.1 - 7.4, the primary difference here being that only basic SP factors are added to smaller pre-existing BFFSP designs.

Theorem 7.5 Let $D\left(n_{1}, n_{2} ; k_{1}, k_{2} ; b_{1}, b_{2}\right)$ be a $2^{\left(n_{1}+n_{2}\right)-\left(k_{1}+k_{2}\right) \pm\left(b_{1}+b_{2}\right)}$ BFFSP design with WLP $W$. Let $W^{W P}$ be the WLP of $D$ for the words containing only WP factors and pure WP blocking variables. Also, let $W^{S P}$ be the WLP of $D$ for words containing any $S P$ factors or separators. There exists a $D\left(n_{1}, n_{2}+2^{k_{2}+b_{2}}-1 ; k_{1}, k_{2} ; b_{1}, b_{2}\right) B F F S P$ design with $W L P W^{W P}+\operatorname{lag}\left(W^{S P}, 2^{k_{2}+b_{2}}\right)$.

Proof: This proof follows in the manner of Theorem 7.1 but here we are adding only SP factors.

Let $D_{1}$ be a $D\left(n_{1}, n_{2} ; k_{1}, k_{2} ; b_{1}, b_{2}\right)$ design represented by $(\mathbf{M}, \mathbf{f})$. Let $\mathbf{f}^{*}$ be a split-plot frequency matrix with $2^{d}-2^{k_{2}+b_{2}}$ columns of the form $(0,0,0,0)^{\prime}$ and $2^{k_{2}+b_{2}}-1$ columns of the form $(0,0,1,0)^{\prime}$. This form for $\mathbf{f}^{*}$ implies that we will be exclusively adding SP factors to the existing design $D_{1}$. Therefore, the $2^{k_{2}+b_{2}}-1$ columns of the form $(0,0,1,0)^{\prime}$ are assigned to each of the $2^{k_{2}+b_{2}}-1$ columns in $A_{1}$ of $\mathbf{M}$ (7.3). Now, denote the BFFSP design corresponding to $\left(\mathbf{M}, \mathbf{f}+\mathbf{f}^{*}\right)$ as $D_{2}$. Since, $\sum\left(f_{1, i},+f_{1, i}^{*}\right)=n_{1}, \sum\left(f_{2, i},+f_{2, i}^{*}\right)=b_{1}, \sum\left(f_{3, i},+f_{3, i}^{*}\right)=n_{2}+2^{k_{2}+b_{2}}-1$ and $\sum\left(f_{4, i},+f_{4, i}^{*}\right)=b_{2}$, then $D_{2}$ is a $D\left(n_{1}, n_{2}+2^{k_{2}+b_{2}}-1 ; k_{1}, k_{2} ; b_{1}, b_{2}\right)$ BFFSP design.

We must now demonstrate that the WLP of the $D\left(n_{1}, n_{2}+2^{k_{2}+b_{2}}-1 ; k_{1}, k_{2} ; b_{1}, b_{2}\right)$ design is indeed $W^{W P}+\operatorname{lag}\left(W^{S P}, 2^{k_{2}+b_{2}}\right)$. Similar to the matrices $\mathbf{M}_{\mathbf{2}}(7.1)$ and $\mathbf{M}$,

$$
\mathbf{M}_{3}=\left(\begin{array}{cc}
I_{k_{2}+b_{2}} & B_{2} \\
B_{2}^{\prime} & B_{2}^{\prime} B_{2}
\end{array}\right)
$$


can be viewed as equivalent to a Hadamard matrix, without the identity column and row of 1's. As a consequence of this equivalence, we conclude that there are $2^{k_{2}+b_{2}-1}$ 1 's in each row of $\mathbf{M}_{\mathbf{3}}$. One can also infer the existence of $2^{k_{2}+b_{2}-1} 1$ 's in each of the rows corresponding to $\left(C_{2}^{\prime}, C_{2}^{\prime} B_{2}\right)$ in $A_{1}$ since these rows are nonzero linear combinations of the rows of the "Hadamard" matrix, $\mathrm{M}_{3}$, over $G F(2)$. We then see that by assigning new SP factors to each of the columns in $A_{1}$, the word-length of each word in the DCS containing at least one SP factor or separator is increased by $2^{k_{2}+b_{2}-1}$. (This implies that the word-lengths of words containing only WP factors or pure WP blocking variables remains unchanged.)

Finally, let $W^{S P}=\left(A_{3}, A_{3.5}, A_{4}, A_{4.5}, \ldots, A_{n_{1}+n_{2}+1.5}\right)$ represent the WLP of those words in a $2^{\left(n_{1}+n_{2}\right)-\left(k_{1}+k_{2}\right) \pm\left(b_{1}+b_{2}\right)}$ BFFSP design containing any SP factors or separators. For an increase of $2^{k_{2}+b_{2}-1}$ in the length of each of these words, we must then move each entry, $A_{i}, 2 * 2^{k_{2}+b_{2}-1}=2^{k_{2}+b_{2}}$ positions to the right in $W^{S P}$ as a consequence of both the increased word-length and the presence of fractional resolution in the definition of $W^{S P}$. We see that in comparison to $W^{S P}$ for $D_{1}, W^{S P}$ for $D_{2}$ must have $2^{b_{2}+k_{2}}$ leading zeroes so that $D_{2}$ 's WLP becomes $W^{W P}+\operatorname{lag}\left(W^{S P}, 2^{k_{2}+b_{2}}\right)$.

Example 7.3.2 Consider a 16-run $2^{(3+2)-(1+0) \pm(0+1)}$ BFFSP design with generators $C=A B$ and $\delta_{1}=A B p q$. For this design $W=(1,0,0,1,0,1)$ where $W^{W P}=$ $(1,0,0,0,0,0)$ and $W^{S P}=(0,0,0,1,0,1)$. Theorem 7.5 guarantees the existence of $a$ 32-run $2^{(3+3)-(1+0) \pm(0+1)}$ BFFSP design with $W L P, W=(1,0,0,0,0,1,0,1)$, where $W^{W P}$ remains unchanged and $W^{S P}=(0,0,0,0,0,1,0,1)$.

Theorem 7.6 Let $R\left(n_{1}, n_{2} ; k_{1}, k_{2} ; b_{1}, b_{2}\right)$ be the maximum resolution for a $2^{\left(n_{1}+n_{2}\right)-\left(k_{1}+k_{2}\right) \pm\left(b_{1}+b_{2}\right)}$ BFFSP design and let $R^{S P}\left(n_{1}, n_{2} ; k_{1}, k_{2} ; b_{1}, b_{2}\right)$ be the maximum resolution for the words containing any $S P$ factors or separators. Then $R^{S P}\left(n_{1}, n_{2}+\right.$ $\left.2^{k_{2}+b_{2}}-1 ; k_{1}, k_{2} ; b_{1}, b_{2}\right) \geq 2^{k_{2}+b_{2}-1}+R^{S P}\left(n_{1}, n_{2} ; k_{1}, k_{2} ; b_{1}, b_{2}\right)$.

Proof : Let $D_{1}$ be a maximum resolution $D\left(n_{1}, n_{2} ; k_{1}, k_{2} ; b_{1}, b_{2}\right)$ design represented by $(\mathbf{M}, \mathbf{f})$ and let $R^{S P}\left(n_{1}, n_{2} ; k_{1}, k_{2} ; b_{1}, b_{2}\right)$ be the maximum resolution for the words containing any SP factors or separators. Also, let $D_{2}$ be a BFFSP design corresponding to $\left(\mathbf{M}, \mathbf{f}+\mathbf{f}^{*}\right), \mathbf{f}^{*}$ being defined in the proof of Theorem 7.5. By Theorem 7.5, $D_{2}$ is a $D\left(n_{1}, n_{2}+2^{k_{2}+b_{2}}-1 ; k_{1}, k_{2} ; b_{1}, b_{2}\right)$ design with WLP $W^{W P}+\operatorname{lag}\left(W^{S P}, 2^{k_{2}+b_{2}}\right)$. Therefore, $R^{S P}\left(n_{1}, n_{2} ; k_{1}, k_{2} ; b_{1}, b_{2}\right)$ of a maximum resolution $D\left(n_{1}, n_{2}+2^{k_{2}+b_{2}}-\right.$ $\left.1 ; k_{1}, k_{2} ; b_{1}, b_{2}\right)$ design must be at least $2^{k_{2}+b_{2}-1}+R^{S P}\left(n_{1}, n_{2} ; k_{1}, k_{2} ; b_{1}, b_{2}\right)$. 
Theorem 7.7 establishes the periodicity property for the maximum resolution of words in a design's DCS containing any SP factors or separators. Theorem 7.7 and its proof follow in a fashion similar to that of Theorem 7.3.

Theorem 7.7 For any fixed $n_{1}, n_{2}, k_{1}, k_{2}, b_{1}$ and $b_{2}$ there exists $L_{2}$ such that $\forall l>L_{2}$, $R^{S P}\left(n_{1}, n_{2}+l\left(2^{k_{2}+b_{2}}-1\right) ; k_{1}, k_{2} ; b_{1}, b_{2}\right)=2^{k_{2}+b_{2}-1}+R^{S P}\left(n_{1}, n_{2}+(l-1)\left(2^{k_{2}+b_{2}}-\right.\right.$ $\left.1) ; k_{1}, k_{2} ; b_{1}, b_{2}\right)$. That is, there exists $L_{2}$ such that $\forall l>L_{2}$, the maximum resolution for words in the DCS of a BFFSP design containing any SP factors or separators is periodic.

Proof : The proof is by contradiction. Assume that there are infinitely many $l_{2, i}$ such that,

$$
\begin{aligned}
& R^{S P}\left(n_{1}, n_{2}+l_{2, i}\left(2^{k_{2}+b_{2}}-1\right) ; k_{1}, k_{2} ; b_{1}, b_{2}\right) \\
& \quad \geq 2^{k_{2}+b_{2}-1}+R^{S P}\left(n_{1}, n_{2}+\left(l_{2, i}-1\right)\left(2^{k_{2}+b_{2}}-1\right) ; k_{1}, k_{2} ; b_{1}, b_{2}\right)+1 .
\end{aligned}
$$

Assuming (7.14) we then have an increasing sequence of $l_{2}$ 's, say $\left\{l_{2, i}\right\}_{i=0}^{\infty}$ such that (7.14) is true. Now by Theorem 7.6 we have that,

$$
\begin{aligned}
& R^{S P}\left(n_{1}, n_{2}+\left(l_{2, i}-1\right)\left(2^{k_{2}+b_{2}}-1\right) ; k_{1}, k_{2} ; b_{1}, b_{2}\right) \\
& \quad \geq 2^{k_{2}+b_{2}-1}+R^{S P}\left(n_{1}, n_{2}+\left(l_{2, i}-2\right)\left(2^{k_{2}+b_{2}}-1\right) ; k_{1}, k_{2} ; b_{1}, b_{2}\right) .
\end{aligned}
$$

Substituting this result into (7.14) we obtain,

$$
\begin{aligned}
& R^{S P}\left(n_{1}, n_{2}+l_{2, i}\left(2^{k_{2}+b_{2}}-1\right) ; k_{1}, k_{2} ; b_{1}, b_{2}\right) \\
& \quad \geq 2 * 2^{k_{2}+b_{2}-1}+R^{S P}\left(n_{1}, n_{2}+\left(l_{2, i}-2\right)\left(2^{k_{2}+b_{2}}-1\right) ; k_{1}, k_{2} ; b_{1}, b_{2}\right)+1 .
\end{aligned}
$$

Using (7.14) and Theorem 7.6,

$$
\begin{aligned}
& R^{S P}\left(n_{1}, n_{2}+l_{2, i}\left(2^{k_{2}+b_{2}}-1\right) ; k_{1}, k_{2} ; b_{1}, b_{2}\right) \\
& \quad \geq\left(l_{2, i}-l_{2, i-1}\right) 2^{k_{2}+b_{2}-1}+R^{S P}\left(n_{1}, n_{2}+l_{2, i-1}\left(2^{k_{2}+b_{2}}-1\right) ; k_{1}, k_{2} ; b_{1}, b_{2}\right)+2
\end{aligned}
$$

Eventually,

$$
\begin{aligned}
& R^{S P}\left(n_{1}, n_{2}+l_{2, i}\left(2^{k_{2}+b_{2}}-1\right) ; k_{1}, k_{2} ; b_{1}, b_{2}\right) \\
& \quad \geq l_{2, i} 2^{k_{2}+b_{2}-1}+R^{S P}\left(n_{1}, n_{2} ; k_{1}, k_{2} ; b_{1}, b_{2}\right)+i .
\end{aligned}
$$


Now, Bingham and Sitter (1999b) showed that,

$$
R^{S P}\left(n_{1}, n_{2} ; k_{1}, k_{2}\right) \leq \frac{2^{k_{2}-1}}{2^{k_{2}}-1}\left(n_{1}+n_{2}\right) .
$$

It is obvious then that

$$
R^{S P}\left(n_{1}, n_{2} ; k_{1}, k_{2} ; b_{1}, b_{2}\right) \leq \frac{2^{k_{2}+b_{2}-1}}{2^{k_{2}+b_{2}}-1}\left(n_{1}+n_{2}\right) .
$$

It follows from (7.16) that

$$
\begin{aligned}
R^{S P} & \left(n_{1}, n_{2}+l_{2, i}\left(2^{k_{2}+b_{2}}-1\right) ; k_{1}, k_{2} ; b_{1}, b_{2}\right) \\
& \leq \frac{2^{k_{2}+b_{2}}-1}{2^{k_{2}+b_{2}}-1}\left(n_{1}+n_{2}+l_{2, i}\left(2^{k_{2}+b_{2}}-1\right)\right) \\
& =\frac{2^{k_{2}+b_{2}-1}}{2^{k_{2}+b_{2}}-1}\left(n_{1}+n_{2}\right)+l_{2, i} 2^{k_{2}+b_{2}-1}
\end{aligned}
$$

From (7.15)

$$
\begin{aligned}
& l_{2, i} 2^{k_{2}+b_{2}-1} \\
& \quad \leq R^{S P}\left(n_{1}, n_{2}+l_{2, i}\left(2^{k_{2}+b_{2}}-1\right) ; k_{1}, k_{2} ; b_{1}, b_{2}\right)-R^{S P}\left(n_{1}, n_{2} ; k_{1}, k_{2} ; b_{1}, b_{2}\right)-i
\end{aligned}
$$

and from (7.17)

$$
\begin{aligned}
& l_{2, i} 2^{k_{2}+b_{2}-1} \\
& \quad \geq R^{S P}\left(n_{1}, n_{2}+l_{2, i}\left(2^{k_{2}+b_{2}}-1\right) ; k_{1}, k_{2} ; b_{1}, b_{2}\right)-\frac{2^{k_{2}+b_{2}-1}}{2^{k_{2}+b_{2}}-1}\left(n_{1}+n_{2}\right) .
\end{aligned}
$$

Therefore, using (7.18) and (7.19) and multiplying through by -1 , we obtain,

$$
\frac{2^{k_{2}+b_{2}-1}}{2^{k_{2}+b_{2}}-1}\left(n_{1}+n_{2}\right) \geq R^{S P}\left(n_{1}, n_{2} ; k_{1}, k_{2} ; b_{1}, b_{2}\right)+i .
$$

This is not true for $i \rightarrow \infty$, thereby establishing the contradiction.

Theorem 7.8 Let $D$ be a $2^{\left(n_{1}+n_{2}\right)-\left(k_{1}+k_{2}\right) \pm\left(b_{1}+b_{2}\right)}$ BFFSP design with WLP W. Let $W^{W P}$ be the WLP of $D$ for words containing only WP factors and pure WP blocking variables. Similarly, let $W^{S P}$ be the WLP of $D$ for words containing any SP factors or separators. For any fixed $n_{1}, n_{2}, k_{1}, k_{2}, b_{1}$ and $b_{2}$ there exists $Q_{2}$ such that $\forall q>$ $Q_{2}$, if the MA $2^{\left\{n_{1}+\left[n_{2}+(q-1)\left(2^{k_{2}+b_{2}}-1\right)\right]\right\}-\left(k_{1}+k_{2}\right) \pm\left(b_{1}+b_{2}\right)}$ BFFSP design has WLP W, then the MA $2^{\left\{n_{1}+\left[n_{2}+q\left(2^{k_{2}+b_{2}}-1\right)\right]\right\}-\left(k_{1}+k_{2}\right) \pm\left(b_{1}+b_{2}\right)}$ BFFSP design has WLP $W^{W P}+$ $\operatorname{lag}\left(W^{S P}, 2^{k_{2}+b_{2}}\right)$. 
Proof : Consider a $2^{\left\{n_{1}+\left[n_{2}+q_{2, i}\left(2^{k_{2}+b_{2}}-1\right)\right]\right\}-\left(k_{1}+k_{2}\right) \pm\left(b_{1}+b_{2}\right)}$ BFFSP design. With each unit increase in the value of $q_{2, i}$, the words in this design's DCS possessing at least one SP factor or separator will increase in length by $2^{k_{2}+b_{2}-1}$. Clearly then, when $q_{2, i}$ becomes large, the $2^{k_{1}+b_{1}}-1$ shortest words in the design's DCS will be those containing only WP factors and pure WP blocking variables. Therefore, for sufficiently large $q_{2, i}$, a MA BFFSP design must have its WP factors and WP blocking variables arranged as a MA $2^{n_{1}-k_{1} \pm b_{1}}$ BFF. This is assumed for the remainder of the proof.

The remainder of this proof parallels that of Theorem 7.4. Let $v_{q_{2, i}}$ be the number of shortest length words containing at least one SP factor or separator in the DCS of the $2^{\left\{n_{1}+\left[n_{2}+q_{2, i}\left(2^{k_{2}+b_{2}}-1\right)\right]\right\}-\left(k_{1}+k_{2}\right) \pm\left(b_{1}+b_{2}\right)}$ BFFSP design where $q_{2, i}<q_{2, j} \forall i<j$. Using Theorems 7.5 - 7.7 and the definition of MA for BFFSP designs (Definition 3.2.1), $v_{q_{2, i}}$ has the property, $v_{q_{2, i}} \geq v_{q_{2, j}}$ for $q_{2, i} \leq q_{2, j}$. That is, there is a positive integer $v_{2}$ such that $v_{q_{2, i}}=v_{2}$ for sufficiently large $i$. By the periodicity of maximum resolution (Theorem 7.7) the limit to the number of words of shortest length is nonzero.

Recall that the MA criterion sequentially minimizes successively larger words in a design's WLP. This fact, along with results motivated by Theorems $7.5-7.7$, would enable us to similarly construct sequences for the number of words with the second shortest word-length, third shortest word-length, and so on, with each of these sequences having a nonegative integer as a limit. Note that if we have only finitely many sequences there will be a finite $Q_{2}>L_{2}\left(L_{2}\right.$ from Theorem 7.7) such that for $q \geq Q_{2}, W_{q_{2, i}}^{S P}=\operatorname{lag}\left(W^{S P}, 2^{k_{2}+b_{2}} q_{2, i}\right)$, where $W_{q_{2, i}}^{S P}$ and $W^{S P}$ are the SP WLPs of MA $2^{\left\{n_{1}+\left[n_{2}+q_{2, i}\left(2^{k_{2}+b_{2}}-1\right)\right]\right\}-\left(k_{1}+k_{2}\right) \pm\left(b_{1}+b_{2}\right)}$ and $2^{\left(n_{1}+n_{2}\right)-\left(k_{1}+k_{2}\right) \pm\left(b_{1}+b_{2}\right)}$ designs, respectively. The final justification for the $2^{k_{2}+b_{2}}$ leading zeroes in $W_{q_{2, i}}^{S P}$ follows an argument identical to that in Theorem 7.1.

What we must establish now is that there are only finitely many sequences that need to be constructed for a MA $2^{\left\{n_{1}+\left[n_{2}+q_{2, i}\left(2^{k_{2}+b_{2}}-1\right)\right]\right\}-\left(k_{1}+k_{2}\right) \pm\left(b_{1}+b_{2}\right)}$ design. To do this we show that the word-lengths of words containing at least one SP factor or separator lie in an interval of finite length for any $q_{2, i}$. Trivially, we know that the length of the shortest word in the DCS of any $2^{\left(n_{1}+n_{2}\right)-\left(k_{1}+k_{2}\right) \pm\left(b_{1}+b_{2}\right)}$ BFFSP design must be bounded below by 1 . In using Theorem 7.8 we will add $q_{2, i}\left(2^{k_{2}+b_{2}}-1\right)$ SP treatment factors to the design. Therefore, the length of the shortest word must be bounded below by $1+q_{2, i}\left(2^{k_{2}+b_{2}-1}\right)$.

To find an upper bound on the longest word containing at least one SP factor or 
separator, we again utilize (as in Theorem 7.4) an adaptation of the identity due to Brownlee, Kelly and Loraine (1948). This adaptation is,

$$
\sum i A_{i}=\left\{n_{1}+\left[n_{2}+q_{2, i}\left(2^{k_{2}+b_{2}}-1\right)\right]\right\} 2^{d-1},
$$

where (7.20) gives the sum of all the word-lengths in the DCS of a $2^{\left\{n_{1}+\left[n_{2}+q_{2, i}\left(2^{k_{2}+b_{2}}-1\right)\right]\right\}-\left(k_{1}+k_{2}\right) \pm\left(b_{1}+b_{2}\right)}$ design. However, we are only interested in the sum of the word-lengths containing at least one SP factor or separator. Therefore, we need to subtract from (7.20) the sum of the word-lengths in the DCS arising from those words consisting exclusively of WP factors and WP blocking variables. This sum of the WP word-lengths is

$$
\sum i A_{i}=n_{1} 2^{k_{1}+b_{1}-1}
$$

and when subtracted from (7.20) we obtain

$$
\sum i A_{i}=\left\{n_{1}+\left[n_{2}+q_{2, i}\left(2^{k_{2}+b_{2}}-1\right)\right]\right\} 2^{d-1}-n_{1} 2^{k_{1}+b_{1}-1},
$$

the sum of the SP word-lengths. If the resolution of SP words is denoted by $R^{S P}$, the longest possible word-length, $U$, has an upper bound given by,

$$
\begin{aligned}
U \leq & \left\{n_{1}+\left[n_{2}+q_{2, i}\left(2^{k_{2}+b_{2}}-1\right)\right]\right\} 2^{d-1}-n_{1} 2^{k_{1}+b_{1}-1} \\
& -\left[\left(2^{d}-1\right)-\left(2^{k_{1}+b_{1}}-1\right)-1\right] R^{S P} \\
= & \left\{n_{1}+\left[n_{2}+q_{2, i}\left(2^{k_{2}+b_{2}}-1\right)\right]\right\} 2^{d-1}-n_{1} 2^{k_{1}+b_{1}-1} \\
& -\left[\left(2^{d}-2^{k_{1}+b_{1}}-1\right)\right] R^{S P} .
\end{aligned}
$$

Using Theorem 7.6, a MA $2^{\left\{n_{1}+\left[n_{2}+q_{2, i}\left(2^{k_{2}+b_{2}}-1\right)\right]\right\}-\left(k_{1}+k_{2}\right) \pm\left(b_{1}+b_{2}\right)}$ design has the following bound for its shortest SP word-length,

$$
\begin{aligned}
& R^{S P}\left(n_{1}, n_{2}+q_{2, i}\left(2^{k_{2}+b_{2}}-1\right) ; k_{1}, k_{2} ; b_{1}, b_{2}\right) \\
& \quad \geq q_{2, i} 2^{k_{2}+b_{2}-1}+R^{S P}\left(n_{1}, n_{2} ; k_{1}, k_{2} ; b_{1}, b_{2}\right) .
\end{aligned}
$$

Let $R_{0}$ be the maximum resolution for the design with $q_{2, i}=0$. Then, from (7.22) and (7.23), the longest SP word-length is bounded above by,

$$
\begin{gathered}
U \leq\left\{n_{1}+\left[n_{2}+q_{2, i}\left(2^{k_{2}+b_{2}}-1\right)\right]\right\} 2^{d-1}-n_{1} 2^{k_{1}+b_{1}-1} \\
-\left[\left(2^{d}-2^{k_{1}+b_{1}}-1\right)\right]\left(q_{2, i} 2^{k_{2}+b_{2}-1}+R_{0}\right) .
\end{gathered}
$$


This simplifies to,

$$
U \leq\left(n_{1}+n_{2}\right) 2^{d-1}-n_{1} 2^{k_{1}+b_{1}-1}+q_{2, i} 2^{k_{2}+b_{2}-1}-R_{0}\left(2^{d}-2^{k_{1}+b_{1}}-1\right) .
$$

Hence, the SP word-lengths of a MA $2^{\left\{n_{1}+\left[n_{2}+q_{2, i}\left(2^{\left.\left.\left.k_{2}+b_{2}-1\right)\right]\right\}-\left(k_{1}+k_{2}\right) \pm\left(b_{1}+b_{2}\right)} \text { design must }\right.\right.\right.}$ be in the interval,

$$
\left[1+q_{2, i} 2^{k_{2}+b_{2}-1},\left(n_{1}+n_{2}\right) 2^{d-1}-n_{1} 2^{k_{1}+b_{1}-1}+q_{2, i} 2^{k_{2}+b_{2}-1}-R_{0}\left(2^{d}-2^{k_{1}+b_{1}}-1\right)\right] .
$$

The length of this interval is independent of $q_{2, i}$ and therefore, is of finite length for any $q_{2, i}$ which proves the theorem.

\subsubsection{Comments Regarding Theorems 7.4 and 7.8}

In applying Theorems 7.4 and 7.8 it is required that one know the values of $Q_{1}$ and $Q_{2}$, respectively, for which the MA criterion becomes periodic. It is useful to note that $Q_{1}$ and $Q_{2}$ will vary for different values of $n_{1}, n_{2}, k_{1}, k_{2}, b_{1}$ and $b_{2}$. This implies that the MA criterion becomes periodic at different times for different BFFSP designs. Therefore, establishing appropriate values for $Q_{1}$ and $Q_{2}$ requires that one separately consider each BFFSP design in the MA BFFSP design catalog.

The task of finding $Q_{1}$ and $Q_{2}$ is essentially a linear-integer programming problem. In the FFSP context, Bingham (1998) provides $Q_{1}$ and $Q_{2}$ values for a limited number of FFSP designs. Extensions of this approach to the BFFSP setting should be straightforward, albeit perhaps time consuming. It is not our intention, at this point, to develop a solution to this problem and so it remains an open research area. 


\section{Chapter 8}

\section{Optimal BFFSP Experiments for Robust Parameter Design}

Wu and Hamada (2000, p.436) define robust parameter design (RPD) as "statistical / engineering methodology that aims at reducing the performance variation of a system (that is, a product or process) by choosing the setting of its control factors to make it less sensitive to noise variation." Control factors inherit their name as a consequence of an experimenter's ability to control them while performing the experiment. In RPD the majority of variability of a process around a target value or response is thought to be caused by a second set of uncontrollable noise factors. Noise variables are required to be controlled at the design level for the purpose of experimentation but are assumed to be uncontrollable at the production level. It is this lack of control at the production level that transmits variability to the process. Of primary interest to the experimenter is the impact of noise variation on the selection of control factor settings. In an effort to ascertain control factor settings that are robust to noise, interest often focuses upon the estimation of control $\times$ noise $(\mathrm{CN})$ interactions. In RPD, one wishes "to find the level settings of the control factors that result in the flattest line across the levels of the noise factors" (Loeppky and Sitter, 2002). A flat line for a control factor setting implies that the response is unaffected by the noise factor.

The current drive for quality improvement via RPD owes itself largely to the pioneering work of Genichi Taguchi in the 1980's (Taguchi and Wu, 1980; Taguchi, 1986; Taguchi, 1987). Although much of Taguchi's work has been constructively 
criticized, it in effect laid the foundation for the modern framework of RPD.

In recent years, RPD has undergone refinements. Myers and Montgomery (2002) summarize methods by which one may use response surface models for the development of a robust process or product. Wolfinger and Tobias (1998) present an extension of the general linear mixed model methodology for simultaneously modeling location effects, dispersion effects and random effects in RPD. Wu and Hamada (2000) present a thorough summary of the location and dispersion modeling approach as well as the response modeling approach for RPD.

The papers by Box and Jones (1992) and Bisgaard (2000) are of high interest in that they demonstrate the usefulness of split-plot designs for robust parameter experimentation. In these papers the authors show that split-plot designs are of considerable value in that they provide the experimenter with efficient estimates of parameter effects while yet possibly allowing for savings in terms of experimental effort. For example, Taguchi's approach to RPD required the use of cross arrays, where a cross array is a cross-product of two designs - an inner array containing the control factors and an outer array containing the noise factors. Many authors have noted, including Box and Jones (1992), that "except in situations where both these arrays are small, this arrangement may involve a prohibitively large amount of experimental work." In an attempt to reduce such work, split-plot designs are frequently performed.

Cox (1958) and Box and Jones (1992) note that split-plot designs are particularly useful when one or more factors are classification (WP) factors. Cox (1958) defines classification factors as experimental factors which are included in the design "to see if they modify the action of other factors or indicate how the other factors work." Main effects of the classification factors are not of particular interest but are included to examine potential interaction with other factors. Cox (1958) also noted that lower precision is acceptable in the estimation of these classification factors so that the precision of the other factors and their interactions with classification factors may be increased. By thinking of the classification factors as noise (environmental) factors, many researchers have opted for the use of split-plot designs in robust parameter experiments.

Until now, the primary criterion we have considered when rank-ordering BFFSP designs has been the MA criterion. Recall that the MA criterion (Definition 3.2.1) 
seeks to minimize short wordlengths in a design's DCS so that low-order effects of interest may be clearly estimated. Although the MA criterion in Definition 3.2.1 provides an experimenter with reasonable designs for estimating key low-order effects, we will demonstrate that it is unable to correctly rank-order designs for the purpose of RPD. Deficiencies in the MA criterion may be brought to light when considering the differing order of effect importance in RPD. For example, it is understood that in RPD, the CN 2fi's are the 2fi's of primary interest in analysis. Recall that in RPD we attempt to select control factor settings which dampen the influence of any noise factors on the response or process. This selection of appropriate control factor settings is often done by visual inspection of CN interaction plots. Consequently, control $\times$ control $(\mathrm{CC})$ and noise $\times$ noise $(\mathrm{NN})$ interactions are not as highly valued when selecting appropriate control factor level settings. Since the MA criterion, as presently defined, cannot distinguish between $\mathrm{CC}, \mathrm{NN}$ and $\mathrm{CN} 2 \mathrm{f}$ 's, it is not ideal for rank-ordering robust designs.

In an attempt to resolve the deficiencies of the MA criterion in the two-level FFSP RPD setting, Bingham and Sitter (2003) revise the notions of resolution and MA. They then use their adapted definitions of resolution and MA to assist in their FFSP RPD ranking procedure. We reserve a more detailed discussion regarding their approach for Section 8.1.2.

The primary objective of this chapter is to formulate a methodology by which one may construct, and subsequently rank, "good" two-level BFFSP designs for the purpose of robust parameter experimentation. In doing this, the following steps will be taken:

1. Develop an effect ranking scheme.

2. Given an effect ranking scheme, construct a program that searches for optimal BFFSP RPDs.

3. Construct tables of optimal BFFSP RPDs.

\subsection{Effect Orderings for FF and FFSP RPDs}

This section provides an overview of two current approaches for ranking two-level FF and FFSP RPDs. In this context we will consider research conducted by Wu and 
Hamada (2000) and Bingham and Sitter (2003).

\subsubsection{Effect Ordering for FF RPDs}

Wu and Hamada (2000, p.462) acknowledge the inadequacy of current definitions for resolution and MA for ranking FF RPDs. They suggest that for FF RPDs the importance of effects should be arranged in the following descending order of importance:

(i) CN 2fi's, C and N main effects;

(ii) CC 2fi's and control $\times$ control $\times$ noise $(\mathrm{CCN}) 3$ fi's;

(iii) NN 2fi's.

The CN 2fi's and control main effects are the most important effects in the preceding list because they may be employed in parameter design optimization procedures. (These optimization procedures are detailed in Wu and Hamada (2000, pp.446-451) and consist of the "two-step procedure for nominal-the-best problems" and the "twostep procedure for larger-the-better and smaller-the-better problems".) The rationale for including the noise main effects in (i) is that, although their levels cannot be adjusted in parameter design optimization, their magnitude (due to their low-order) can make it difficult to assess the significance of other effects that are aliased with them. Further explanation for the preceding effect ordering may be found in Wu and Hamada (2000, p.463).

The following example, given by Chen et al. (1993), illustrates the inadequacy of the resolution and MA criteria for ranking FF RPDs.

Example 8.1.1 Consider a $2^{6-2} F F$ design where $A, B$ and $C$ are three control factors and $r, s$ and $t$ are three noise factors. The resolution $4 M A$ design, $D_{1}$, has $D C S I=A B C r=B C s t=A r s t$, such that the six me's, $A, B, C, r, s$ and $t$ are clear. Now consider the resolution 3 design, $D_{2}$, with $D C S I=A B C r=r s t=A B C s t$. This lower-resolution design yields the following nine clear effects-A, B, C, As, Bs, Cs, At, Bt and Ct. Assuming the validity of the preceding effect ordering principle, $D_{2}$ has higher estimation capacity than the $M A$ design, $D_{1}$, since 9 effects are of rank "(i)" for $D_{2}$ whereas only 6 effects are of rank "(i)" for $D_{1}$. 


\subsubsection{Effect Ordering for FFSP RPDs}

For screening experiments we know that the hierarchical ordering principle states that lower-order effects are more likely to be important than higher-order effects and that effects of the same order are equally likely to be important. We have also seen that in RPD, not all effects of the same order are of equal interest (for example, CN Vs. NN 2fi's). In the context of FFSP RPDs, Bingham and Sitter (2003) construct an effect ranking scheme that is a compromise between the notions of "likely significance" of effects and "effect interest". To understand the compromise between these two issues Bingham and Sitter (2003) first provide the following rankings (see Table 8.1).

Table 8.1: Effect Rankings for FFSP RPDs

\begin{tabular}{cll}
\hline \hline Ranking & Likely Significance & Interest \\
\hline (i) & $C, N$ & $C, C N$ \\
(ii) & $C C, C N, N N$ & $C C, C C N, C N N$ \\
(iii) & $C C C, C C N, C N N, N N N$ & $C C C$ \\
\hline
\end{tabular}

The "likely significance" ranking, in Table 8.1 may be explained by way of the hierarchical principle. The "interest" rankings of Table 8.1 are justified by Bingham and Sitter (2003) as follows. First, these authors note that in RPD, the effects of primary interest are the control main effects, the CN 2fi's and the CC 2fi's. Knowledge of the statistical significance of the control main effects and the CC $2 \mathrm{fi}$ 's gives the practitioner the ability to improve the process mean by appropriately adjusting the levels of the significant control effects. In addition, the ability to dampen the effect of the noise factors on the response variability is obtained by a judicious selection of control factor level settings as prescribed by the $\mathrm{CN}$ interaction plots. Since the levels of the noise effects cannot be set in practice, they cannot be directly used to improve the process. Consequently, effect estimates of noise main effects and their interactions are of less interest. Further discussion regarding the usefulness of CC, CCN and CNN interactions in FFSP RPDs is included in Bingham and Sitter (2003).

From Table 8.1, Bingham and Sitter (2003) form their proposed effect ranking scheme, presented in Table 8.2, with word-lengths in parentheses. This ranking scheme provides a compromise betweeen the two issues of likely effect significance 
and effect interest.

Table 8.2: Compromise Ranking Scheme with Associated Word-lengths

\begin{tabular}{cl}
\hline \hline Length & Effects \\
\hline$(1)$ & $C, N$ \\
$(1.5)$ & $C N$ \\
$(2)$ & $C C, N N$ \\
$(2.5)$ & $C C N, C N N$ \\
$(3)$ & $C C C, N N N$ \\
\hline
\end{tabular}

Using the word-lengths from Table 8.2, word-lengths of larger words may be found. With these redefined word-lengths one can simply use the usual definitions of resolution and MA to form catalogs of MA FFSP RPDs. Bingham and Sitter (2003) obtain MA FFSP RPDs for the following two scenarios:

1. Noise factors as WP factors (that is, control factors as SP factors);

2. Control factors as WP factors (that is, noise factors as SP factors).

By way of example these authors demonstrate the superiority of their RPD MA criterion over the usual MA criterion for FFSP screening experiments.

The following example illustrates one instance where the RPD MA criterion selects a FFSP RPD with higher estimation capacity than what would have otherwise been selected using the usual MA criterion for screening experiments.

Example 8.1.2 Consider a $2^{(3+5)-(0+3)} R P D$ with $n_{1}=3$ control factors and $n_{2}=5$ noise factors. One MA FFSP design, $D_{1}$, has $D C S I=A B C p r=A B C q s=A p q t$ $=$ pqrs $=B C q r t=B C p s t=$ Arst. Using the new word-lengths, the MA FFSP RPD, $D_{2}$, has $D C S I=p q r=A B C p s=A B C q t=A B C q r s=A B C p r t=p q s t=r s t$. In $D_{2}$ all $C N$ 2fi's are clear if three factor and higher interactions are negligible. In comparison, the words Apqt and Arst in the DCS of $D_{1}$ cause all CN 2fi's involving $A$ to be aliased with $N N$ 2fi's, which are not of interest but are potentially significant. 


\subsection{Effect Ordering for BFFSP RPDs}

Thus far we looked at the methodology of Wu and Hamada (2000) and Bingham and Sitter (2003) for ranking FF and FFSP RPDs. We will now present an approach for ranking BFFSP designs for the purpose of robust parameter experimentation. In advance of implementing a mathematical or computer algorithm that searches for optimal BFFSP RPDs, we must first develop an effect ranking scheme. This effect ranking scheme should allow one to discriminate between BFFSP RPDs of varying estimation capacity. Table 8.3 presents, in descending order of importance, a ranking scheme for the ordering of low-order effects in BFFSP RPDs. In addition, Table 8.3 includes the ranking of the optimality characteristic, effect precision, associated with each of the low-order effects.

Table 8.3: Effect and Effect Precision Rankings for BFFSP RPDs

\begin{tabular}{cl}
\hline \hline Ranking & Effects / Effect Precision \\
\hline (a) & $C$ \\
(b) & $C N$ \\
(c) & $C C$ \\
(d) & The number of clear $C$ main effects tested against WP error. \\
(e) & The number of clear $C N$ 2fi's tested against WP error. \\
(f) & The number of clear $C C$ 2fi's tested against WP error. \\
\hline
\end{tabular}

Some discussion regarding the ranking scheme of Table 8.3 is necessary. First, by the hierarchical principle, control main effects should be ranked ahead of the $\mathrm{CN}$ and CC 2fi's. Control main effects and CN 2fi's are both of high interest, the former for adjusting the mean of a process and the latter for identifying control factors to make the process robust to noise variation. CC 2fi's are also used to adjust the mean of a process but are less likely to be significant than control main effects-therefore, in terms of importance, we rank CC 2fi's below control and CN effects.

It may also be advantageous to the practitioner to have some knowledge of the precision of these important low-order effects. For this reason, we wish to evaluate each BFFSP design for the number of clear low-order (C, CN and CC) effects that are tested against the WP error. Since control main effects are of highest importance 
we wish to see fewer of these effects tested against WP error than we would for CN and CC 2fi's. Moreover, since CN 2fi's are the primary 2fi's of interest in RPD we also wish to have few clear CN 2fi's tested against the WP error. For these reasons, the number of clear CC 2fi's tested against WP error is of lowest rank in Table 8.3.

While developing the optimality criteria for ranking BFFSP RPDs, we chose not to use an adaptation of the MA criterion. (Recall that Bingham and Sitter (2003) adapted the MA criterion in the search for optimal FFSP RPDs.) The rationale for this choice is two-fold. First, there is some ambiguity in determining what are "appropriate" word-lengths for words in the DCSs of RPDs. In the presence of blocking factors this ambiguity is further magnified. Second, the MA criterion is not able to distinguish between effects of varying precision. Instead, we choose to use a sequential search algorithm in conjunction with the ranking scheme of Table 8.3 in order to select those BFFSP designs having large numbers of clear low-order effects (C, CN and CC) but with few (or none) of them being tested against the WP error.

\subsubsection{Implementation of the Effect Ordering for BFFSP RPDs}

This section outlines one approach for implementation of Table 8.3 in the search for optimal BFFSP RPDs.

For a given BFFSP design with $\left(n_{1}, n_{2} ; k_{1}, k_{2} ; b_{1}, b_{2}\right)$, the optimal BFFSP RPD is obtained by sequential completion of the following steps:

(i) Obtain the set of $2^{\left(n_{1}+n_{2}\right)-\left(k_{1}+k_{2}\right) \pm\left(b_{1}+b_{2}\right)}$ BFFSP designs such that the number of clear control main effects is maximized. Denote this set by $D_{(i)}$;

(ii) From $D_{(i)}$, obtain the set of BFFSP designs such that the number of clear CN 2fi's is maximized. Denote this set by $D_{(i i)}$;

(iii) From $D_{(i i)}$, obtain the set of BFFSP designs such that the number of clear CC 2fi's is maximized. Denote this set by $D_{(i i i)}$;

(iv) From $D_{(i i i)}$, obtain the set of BFFSP designs such that the number of clear control main effects tested against WP error is minimized. Denote this set by $D_{(i v)}$.

(v) From $D_{(i v)}$, obtain the set of BFFSP designs such that the number of clear CN 2fi's tested against WP error is minimized. Denote this set by $D_{(v)}$. 
(vi) From $D_{(v)}$, obtain the set of BFFSP designs such that the number of clear CC 2fi's tested against WP error is minimized. Denote this set by $D_{(v i)}$.

It is extremely likely that the final design set, $D_{(v i)}$, will contain more than one optimal BFFSP RPD. For brevity, the optimal BFFSP RPD catalog will include only one representative design from $D_{(v i)}$ for a given value of $\left(n_{1}, n_{2} ; k_{1}, k_{2} ; b_{1}, b_{2}\right)$.

The following example provides an optimal BFFSP design for the purpose of a robust parameter experiment. Its superiority in terms of estimation capacity over that of a MA BFFSP design (developed for screening purposes) is demonstrated.

Example 8.2.1 Consider a $2^{(2+3)-(0+1) \pm(0+1)}$ BFFSP experiment with $n_{2}=3$ control factors. The optimal (representative) BFFSP RPD, $D_{1}$, has DCS $I=A B p q r=$ $A B p \delta_{1}=q r \delta_{1}$ such that all six $C N$ 2fi's ( $A p, A q, A r, B p, B q$ and $B r$ ) are clear. In comparison, the (representative) MA BFFSP design, $D_{2}$, with DCS $I=A B q r=$ $B p q \delta_{1}=A p r \delta_{1}$ yields only two clear CN 2fi's. For the purpose of robust experimentation, $D_{1}$ is by far superior since it has six clear $C N$ 2fi's. Note that the $M A$ criterion selects $D_{2}$ since it has fewer shorter words than $D_{1}$.

When constructing BFFSP RPDs, two cases will be considered. They are:

1. Noise factors as WP factors (that is, control factors as SP factors);

2. Control factors as WP factors (that is, noise factors as SP factors).

For case (2), criteria (d) and (f) of Table 8.3 need not be explicitly calculated since in this case all control main effects and CC 2fi's are WP effects. These effects are then, by definition, automatically tested against WP error.

\subsubsection{A One-Number Optimality Criterion for BFFSP RPDs}

For practitioners that do not wish to use the sequential approach for optimal BFFSP RPD selection we now present an alternative selection criterion. This criterion is a one-number design summary statistic and is a (weighted) function of criteria (a) (f) in Table 8.3.

Let $x_{\text {total }}$ denote the one-number summary statistic where

$$
x_{t o t a l}=w_{1} x_{(i)}+w_{2} x_{(i i)}+w_{3} x_{(i i i)}+w_{4} x_{(i v)}+w_{5} x_{(v)}+w_{6} x_{(v i)} .
$$


The variables $x_{(i)}, \ldots, x_{(v i)}$ are the values for criterion (a) - (f) (Table 8.3) that a specific BFFSP design possesses. The weights, $w_{1}, \ldots, w_{6}$, reflect the importance that the experimenter wishes to place upon the six optimality criteria. Since larger numbers for criteria (a) - (c) are desirable, it is intuitive to have $w_{1}, w_{2}, w_{3} \geq 0$ where $w_{1} \geq w_{2} \geq w_{3}$. (The latter inequality arises due to the priority ordering given to control main effects, CN interactions and CC interactions in Table 8.3.) In contrast, smaller values for criteria (d) - (f) are desirable so it is useful to have the restriction $w_{4}, w_{5}, w_{6} \leq 0$ where $w_{4} \leq w_{5} \leq w_{6}$. With these restrictions on the $w_{i}(1 \leq i \leq 6)$ we then select the BFFSP design that maximizes $x_{\text {total }}$.

The only occasion where a weight, $w_{i}$, should equal zero is when the experimenter has no interest in the associated criterion. For instance, if the objective was only to count the number of clear control main effects and CN 2fi's that a $2^{\left(n_{1}+n_{2}\right)-\left(k_{1}+k_{2}\right) \pm\left(b_{1}+b_{2}\right)}$ BFFSP design possesses, a weight selection of $w_{1}=w_{2}=1$ and $w_{3}=w_{4}=w_{5}=w_{6}=0$ would be appropriate. That is, in this case no consideration would be given to optimality criteria (c) - (f).

Given the variety of expressions that $x_{\text {total }}$ may take, it is envisioned that the optimal BFFSP RPD determined by the one-number summary statistic could be different than the design selected by the sequential approach of Section 8.2.1. An example illustrating this possibility is given in Section 8.3.

Finally, when comparing BFFSP RPDs having control factors at the WP level, it is reasonable to set $w_{4}=w_{6}=0$ since in this case we know in advance that all control main effects and CC 2fi's will be tested against WP error.

\subsubsection{Overview of the RPD Search Algorithm}

This section provides a brief overview of the structure of the program used to construct optimal BFFSP RPDs.

The program, "RPDsearchmixed.cpp", consists of a main body of code that calls upon four primary functions. These five program elements are now briefly described:

(1) Main Body of Program: This portion prompts the program user to enter all of the relevant variables. For example, $n_{1}, n_{2}, k_{1}, k_{2}, b_{1}$, and $b_{2}$ are all entered at this stage. In addition, the user is prompted to enter the weights, $w_{i},(1 \leq i \leq 6)$ if calculation of $x_{\text {total }}$ is desired. 
The construction of the search-table is also completed at this stage for use in subsequent functions. (See Chapter 5 for a detailed description of the searchtable in the context of BFFSP screening experiments.)

(2) Function "SearchDCSWLP": This function receives (from the main body of the program) the generators for each of the potentially optimal BFFSP RPDs. From these generators, the DCS of the design is constructed using modulus 2 arithmetic. The DCS is then passed back to the main body of the program to be used in subsequent functions.

(3) Function "AliasStructure": A considerable number of calculations are performed by this function. This function first receives the DCS of the BFFSP design currently under consideration. It then calculates the number of clear low-order (C, CN, and CC) effects, using the DCS from "SearchDCSWLP". For each design, this function also calculates the number of clear $\mathrm{C}, \mathrm{CN}$ and $\mathrm{CC}$ effects that are to be tested against WP error. Finally, the one-number optimality criterion (Section 8.2.2) is calculated.

(4) Function "Comparison": This function receives, from "AliasStructure", the number of clear low-order effects that a BFFSP design possesses. Also, it receives information regarding the precision of the low-order effects as well as the value of $x_{\text {total }}$. From these values comparison is made with the values of other BFFSP designs previously calculated. Using Table 8.3 as its guide, this function then selects a representative design that is optimal with respect to these criteria. Note that the optimal designs selected according to Table 8.3 and $x_{\text {total }}$ may differ.

(5) Function "Print": This function prints the DCS of the optimal design(s). As well, this function prints information regarding $x_{t o t a l}$, the number of clear loworder effects, and the precision of the clear low-order effects for the optimal BFFSP robust parameter design(s).

The search algorithm and all other programmable calculations were implemented using Microsoft $t_{\circledast}$ Visual $C^{++\circledast}$ 6.0 Professional Edition. Computer hardware consisted of a Dimension ${ }^{T M} 8100$ Desktop Pentium 4 computer with a $1.40 \mathrm{GHz}$ CPU. 


\subsection{Using the Optimal BFFSP RPD Catalog}

Appendix E contains BFFSP RPDs that are optimal with respect to the sequential ranking scheme of Table 8.3. These designs were constructed via pure WP blocking, separation and mixed blocking. All designs have between 6 and 10 treatment and blocking variables and consist of 16 or 32 runs in either 2 or 4 blocks. Each $2^{\left(n_{1}+n_{2}\right)-\left(k_{1}+k_{2}\right) \pm\left(b_{1}+b_{2}\right)} \mathrm{RPD}$ is abbreviated "Design $=n_{1}, n_{2} ; k_{1}, k_{2} ; b_{1}, b_{2}$ " in order to distinguish between different designs. Within the tables, the designs are presented in ascending order using $n=n_{1}+n_{2}+b_{1}+b_{2}$, the total number of treatment and blocking variables. A - G and p - v are used to denote the WP and SP factors, respectively, with the last letter in each generator representing the added factor. The Greek letters, $\beta_{i}$ and $\delta_{j}$, denote the $i^{\text {th }}$ and $j^{\text {th }}$ pure WP blocking variable and separator, respectively. In columns (a) - (f) we have included a summary of each optimal BFFSP RPD with respect to the six optimality criteria of Table 8.3. In column (g) we have included the value of the one-number optimality criterion, $x_{\text {total }}$, having used the total number of clear $C$ main effects and $C N$ 2fi's as our one-number statistic. (If a different one-number criterion is of interest to the practitioner the search program may be rerun using a different weighting scheme for $x_{\text {total }}$.)

Recall that when constructing BFFSP RPDs we are considering the following two scenarios:

1. Noise factors as WP factors (that is, control factors as SP factors);

2. Control factors as WP factors (that is, noise factors as SP factors).

Consequently, for a given number of runs, each $2^{\left(n_{1}+n_{2}\right)-\left(k_{1}+k_{2}\right) \pm\left(b_{1}+b_{2}\right)}$ design is assessed first by assuming that the noise factors are at the WP level, and then in a separate table, by assuming that the control factors are at the WP level. In the latter case we do not explicitly calculate criteria (d) and (f), since in this case all $\mathrm{C}$ main effects and CC 2fi's will naturally be tested against WP error. Therefore, when control factors are run at the WP level we simply present an assessment of the designs with respect to criteria (a), (b), (c), (e) and (g).

For certain values of $n_{1}, n_{2} ; k_{1}, k_{2} ; b_{1}, b_{2}$ a BFFSP RPD may be optimal with respect to the sequential ranking scheme (Table 8.3) but not with respect to the onenumber criterion, $x_{\text {total }}$. In such cases we present two optimal designs - the design 
that is optimal according to Table 8.3 and the design that is optimal by $x_{\text {total }}$. The latter design is denoted by a "*" and, in the appropriate table, follows immediately after the $2^{\left(n_{1}+n_{2}\right)-\left(k_{1}+k_{2}\right) \pm\left(b_{1}+b_{2}\right)}$ design that is optimal according to the sequential ranking scheme.

Example 8.3.1 Consider a $2^{(2+4)-(0+2) \pm(1+0)}$ design with $n_{2}=4$ control factors. This 16-run design is listed in Table E.1. Its generators are given as $\beta_{1}=A B$, $r=A B p$ and $s=A B q$. This design has four clear $C$ main effects (criterion (a)) and criteria (b) - (f) all have zero values. Using the sequential effect ranking scheme, this design represents the "best" that one can do when the goal is robust experimentation. That is, all other $2^{\left(n_{1}+n_{2}\right)-\left(k_{1}+k_{2}\right) \pm\left(b_{1}+b_{2}\right)}$ designs in $D_{(v i)}$, selected via the sequential ranking scheme, will have four clear $C$ main effects. In addition, all designs in $D_{(v i)}$ will have zero values for criteria (b) - (f).

We have previously mentioned that the optimal BFFSP RPD selected by the onenumber criterion could be different then the optimal design selected by the sequential approach. Such an event occurs here. Using the one-number criterion (where $x_{\text {total }}=$ $x_{(i)}+x_{(i i)}$, the total number of clear $C$ main effects and $C N$ 2fi's) we observe that the $2^{(2+4)-(0+2) \pm(1+0)}$ design with generators $\beta_{1}=A B, r=A B p q$ and $s=A B p q$, has a total of six clear $C$ and $C N$ effects - two more than that of the previous design. (Note that this design is optimal with respect to $x_{\text {total }}$ despite the fact that $r$ is aliased with s.) This "alternative" optimal design is denoted with a "*" and follows, in Table E.1, immediately after the design that is optimal according to the sequential effect ranking scheme.

The designs listed in Appendix $\mathrm{E}$ do not form an exhaustive list of all possible 16 and 32-run BFFSP RPDs. Obviously one could consider designs with values of $n$ (where $n=n_{1}+n_{2}+b_{1}+b_{2}$ ) larger than those considered here. Indeed, even for values of $n$ between 6 and 10 , not all $n_{1}, n_{2} ; k_{1}, k_{2} ; b_{1}, b_{2}$ design combinations have been assessed. The intent of the catalog is to provide a substantial portion of those 16 and 32-run optimal RPDs that experimenters will find useful to perform.

In comparison to the number of designs investigated in the context of MA BFFSP screening experiments, we anticipate that there are more $n_{1}, n_{2} ; k_{1}, k_{2} ; b_{1}, b_{2}$ combinations to consider in the robust design setting. This arises due to the fact that in RPD we no longer restrict ourselves to designs with $R \geq 3$. In the optimal BFFSP 
RPD catalog, many of the designs have $R<3$ and would not have been included in the MA BFFSP design catalog.

Finally, since impractical designs (Section 3.2.2) can occur in the RPD setting we require that $1 \leq b_{2} \leq n_{2}-k_{2}-1$, as we did for MA BFFSP screening experiments. However, since in robust design we no longer have the restriction that $R \geq 3$, we no longer require that $b_{1}$ have an upper bound of $n_{1}-k_{1}-1$ for resolution purposes.

Example 8.3.2 For example, consider a $2^{(3+2)-(0+1) \pm(3+0)}$ design with $n_{2}=2$ control factors. Suppose the generators are $\beta_{1}=A, \beta_{2}=B, \beta_{3}=C$ and $q=A B C p$. The $D C S$ of the design is $I=A \beta_{1}=B \beta_{2}=C \beta_{3}=A B C p q=A B \beta_{1} \beta_{2}=A C \beta_{1} \beta_{3}=$ $B C \beta_{2} \beta_{3}=A B C \beta_{1} \beta_{2} \beta_{3}=B C p q \beta_{1}=A C p q \beta_{2}=A B p q \beta_{3}=C p q \beta_{1} \beta_{2}=B p q \beta_{1} \beta_{3}=$ $A p q \beta_{2} \beta_{3}=p q \beta_{1} \beta_{2} \beta_{3}$, implying that all control main effects and $C N$ 2fi's are clear. Therefore, despite the confounding of noise main effects with blocks, we have constructed a reasonable $R P D$. Clearly, if we were using the restriction $R \geq 3$, (implying $\left.b_{1} \leq n_{1}-k_{1}-1\right)$ this design would not be eligible.

\subsection{Limited Theoretical Results for RPDs}

When selecting a $2^{\left(n_{1}+n_{2}\right)-\left(k_{1}+k_{2}\right) \pm\left(b_{1}+b_{2}\right)}$ design for the purpose of robust experimentation, information regarding the estimation capacity of the design is typically required. In particular, knowledge about the number of clear low-order effects is crucial in selecting the appropriate design. It was seen, in Section 8.2.1, that our search program (implemented via computer) provides us with such knowledge. The objective of this section is to provide a glimpse of the theoretical development necessary to answer questions regarding the estimation capacity of a given RPD. Specifically, we will consider the difficulty in determining, without the aid of a computer, the number of clear CN 2fi's that a given RPD possesses. To do this, we will consider "simple" cases from the FFSP and BFFSP RPD scenarios. It is hoped that the reader will appreciate the need for further research in this area.

The following theorem describes conditions under which all CN 2fi's will be clear for a FFSP design.

Theorem 8.1 Consider a $2^{\left(n_{1}+n_{2}\right)-\left(k_{1}+k_{2}\right)}$ FFSP design in cross array form such that $2^{\left(n_{1}+n_{2}\right)-\left(k_{1}+k_{2}\right)}=2^{\left(n_{1}-k_{1}\right)} \otimes 2^{\left(n_{2}-k_{2}\right)}$, where all $k_{2}$ added $S P$ factors are generated 
exclusively by SP factors. If both the WP and SP arrays are of resolution at least 3 , then all $n_{1} n_{2} C N$ 2fi's will be clear.

Proof : This proof follows from that of Theorem 10.1 (ii) in Wu and Hamada (2000, pp. 460-461).

Without loss of generality, let $2^{\left(n_{1}-k_{1}\right)}=d_{N}$, the noise array and let $2^{\left(n_{2}-k_{2}\right)}=$ $d_{C}$, the control array. Let $u_{1}, \ldots, u_{2^{k_{1}-1}}$ denote the words in the DCS of $d_{N}$ and $v_{1}, \ldots, v_{2^{k_{2}-1}}$ denote the words in the DCS of $d_{C}$. Then $d_{N} \otimes d_{C}$, which is a $2^{\left(n_{1}+n_{2}\right)-\left(k_{1}+k_{2}\right)}$ design, has $2^{k_{1}+k_{2}}-1$ words in its DCS of the form

$$
u_{i}, v_{j}, u_{i} v_{j}
$$

where $i=1, \ldots, 2^{k_{1}}-1$ and $j=1, \ldots, 2^{k_{2}}-1$.

First, note that any of the $n_{1} n_{2}$ CN 2fi's can only appear in $u_{i} v_{j}$ among the words in 8.2. Because $d_{C}$ and $d_{N}$ must both be at least resolution $3, u_{i}$ and $v_{j}$ have at least length 3 . Also, since $u_{i}$ only involves noise factors and $v_{j}$ involves only control factors, this implies that $u_{i} v_{j}$ has length at least 6 . Therefore, any CN $2 \mathrm{fi}$ is aliased with four-factor or higher-order interactions. Also, a CN 2 fi can be aliased with some 3 fi's that involve at least one control factor and at least one noise factor. (See the following example.) Therefore, by definition, each $\mathrm{CN} 2 \mathrm{fi}$ is clear.

Example 8.4.1 Consider a $2^{(3+3)-(1+1)}$ FFSP design with $n_{1}=3$ noise factors. If the added factors are $C=A B$ and $r=p q$ this implies that the DCS is of the form $I$ $=A B C=p q r=A B C p q r$. It is obvious that all $C N$ 2fi's are clear. (For example, $A p$ $=B C p=A q r=B C q r$, implying that $A p$ is clear.) Furthermore, note that all $C N$ 2fi's are clear regardless of whether the noise factors are run at the WP or SP levels.

The following theorem describes conditions under which all CN 2fi's will be clear for a BFFSP design.

Theorem 8.2 Consider a $2^{\left(n_{1}+n_{2}\right)-\left(k_{1}+k_{2}\right) \pm\left(b_{1}+b_{2}\right)}$ BFFSP design in cross array form such that $2^{\left(n_{1}+n_{2}\right)-\left(k_{1}+k_{2}\right) \pm\left(b_{1}+b_{2}\right)}=2^{\left(n_{1}-k_{1}\right) \pm b_{1}} \otimes 2^{\left(n_{2}-k_{2}\right) \pm b_{2}}$, where all $k_{2}$ added $S P$ factors and $b_{2}$ separators are generated exclusively by SP factors. If both the WP and $S P$ arrays are of resolution at least 3, then all $n_{1} n_{2} C N$ 2fi's will be clear.

Proof: Without loss of generality, let $2^{\left(n_{1}-k_{1}\right) \pm b_{1}}=d_{N}$, the noise array and let $2^{\left(n_{2}-k_{2}\right) \pm b_{2}}=d_{C}$, the control array. Let $u_{1}, \ldots, u_{2^{k_{1}+b_{1}-1}}$ denote the words in the 
DCS of $d_{N}$ and $v_{1}, \ldots, v_{2^{k_{2}+b_{2}-1}}$ denote the words in the DCS of $d_{C}$. Then $d_{N} \otimes d_{C}$, which is a $2^{\left(n_{1}+n_{2}\right)-\left(k_{1}+k_{2}\right) \pm\left(b_{1}+b_{2}\right)}$ design, has $2^{k_{1}+k_{2}+b_{1}+b_{2}}-1$ words in its DCS of the form

$$
u_{i}, v_{j}, u_{i} v_{j}
$$

where $i=1, \ldots, 2^{k_{1}+b_{1}}-1$ and $j=1, \ldots, 2^{k_{2}+b_{2}}-1$.

First, note that any of the $n_{1} n_{2}$ CN 2fi's can only appear in $u_{i} v_{j}$ among the words in 8.3. Also, since $d_{C}$ and $d_{N}$ must both be at least resolution $3, u_{i}$ and $v_{j}$ have at least length 3 . In the BFFSP setting, notice that $u_{i}$ may involve both noise factors and pure WP blocking variables. Similarly, $v_{j}$ may involve both control factors and separators. Furthermore, using Equation 3.3, any word containing a blocking variable in the DCSs of $d_{C}$ or $d_{N}$ must be of length at least 3.5. This implies that $u_{i} v_{j}$ has length at least 5.5. Therefore, any $\mathrm{CN} 2 \mathrm{fi}$ is aliased either with treatment $\times$ block interactions (which we assume to be negligible) or with three-factor and higher-order interactions. Regardless, we conclude that each $\mathrm{CN} 2 \mathrm{f}$ is clear.

Example 8.4.2 Consider a $2^{(3+4)-(0+1) \pm(1+1)}$ design with $n_{1}=3$ noise factors. Using the generators $\beta_{1}=A B C, s=p q$ and $\delta_{1}=p r$ we are ensured that $d_{N}$ and $d_{C}$ both have resolution of at least 3. The DCS is given by $I=A B C \beta_{1}=p q s=p r \delta_{1}=$ $A B C p q s \beta_{1}=A B C p r \beta_{1} \delta_{1}=q r s \delta_{1}=A B C q r s \beta_{1} \delta_{1}$. One can verify that all CN 2fi's are aliased with either treatment $\times$ block interactions or with interactions having at least three factors. Therefore, all $C N$ 2fi's are clear.

Theorems 8.1 and 8.2 apply only to those "rare" FFSP and BFFSP RPDs in which the added SP factors and separators are generated exclusively by SP factors. Considerable difficulty arises in predetermining the number of clear CN 2fi's in those FFSP and BFFSP RPDs having WP factors in their SP factor and separator generators. The following theorem describes one scenario where a single WP factor is present within the generator of a lone SP added factor. This result is presented to give the reader an idea as to the complexity in predetermining the number of clear CN 2fi's in FFSP and BFFSP RPDs.

Theorem 8.3 Consider a $2^{\left(n_{1}+n_{2}\right)-\left(k_{1}+k_{2}\right)}$ FFSP design with $k_{1} \geq 0$ and $k_{2}=1$ such that the added SP factor has one WP factor in its generator. For a design with resolution 3 in both $d_{C}$ and $d_{N}$, there can be at most $n_{1} n_{2}-2$ clear CN $2 f$ 's. 
Proof: Without loss of generality let $2^{\left(n_{1}-k_{1}\right)}$ and $2^{\left(n_{2}-k_{2}\right)}$ represent $d_{N}$ and $d_{C}$, respectively. Let $u_{1}, \ldots, u_{2^{k_{1}-1}}$ denote the defining words in the DCS of $d_{N}$ and $v_{1}$ denote the lone word in the DCS of $d_{C}$. Then $d_{N} \times d_{C}$ has $2^{k_{1}+1}-1$ words in its DCS of the form

$$
u_{i}, v_{1}, u_{i} v_{1}
$$

where $i=1, \ldots, 2^{k_{1}}-1$.

Letting $L\left(u_{i}\right)$ denote the length of the word $u_{i}$, we have $\min L\left(u_{i}\right)=3, i=$ $1, \ldots, 2^{k_{1}}-1$. Also, $L\left(v_{1}\right)=3$. Then, since $v_{1}$ contains one WP factor (by assumption) this implies that two CN 2f's are "automatically" unclear.

If the noise (WP) factor in $v_{1}$ is common to a noise factor in one of the $u_{i}$ then $\min L\left(u_{i} v_{1}\right)=\min L\left(u_{i}\right)+L\left(v_{1}\right)-2=3+3-2=4$, so that additional CN 2fi's may be unclear. Therefore, to minimize the number of unclear CN 2fi's arising from the four letter words we must have that $\min L\left(u_{i} v_{1}\right)>4$. This only occurs if the noise factor in $v_{1}$ is not present in any of the $u_{i}$ having length 3 . In this case, the only two unclear CN 2fi's arise from $v_{1}$ and we have at most $n_{1} n_{2}-2$ clear CN 2 fi's.

For FFSP designs having $k_{2}>1$ with several WP factors in each of the added SP factor generators, any theoretical results will require very technical derivations. The level of required mathematical sophistication is further magnified when considering the presence of pure WP blocking variables and separators. In the context of BFFSP RPDs, the use of our search algorithm (implemented via computer) is absolutely necessary in order to circumvent tedious theoretical derivations. Little, if any, work has been completed in this theoretical research area. 


\section{Chapter 9}

\section{The Chrome-Plating Experiment Revisited}

We now wish to address the optimality questions pertaining to the case study first described in Chapter 1. To do this, design issues surrounding the chrome-plating experiment will now be investigated in detail.

\subsection{Three Competing Designs}

Three design scenarios will be considered in this chapter, as illustrated in Figure 9.1. One can think of $E$ as having been obtained from $D$ by elevation of $p$ to $P$ and $S$ as having been obtained from $D$ by separation. $D$ uses four rectifiers and eight days, whereas $E$ and $S$ use two rectifiers and 16 days. All of the designs use the same number of runs, and all are blocked by week. For each scenario the MA design was obtained (using the search-table approach outlined in Chapter 5).

For scenario $D$ two generators are required-one SP factor generator and one WP blocking variable generator. The MA design has $r=A B p q$ as the factor generator and $\beta_{1}=A B C$ as the pure WP blocking generator.

For scenario $E$ three generators are required-one SP factor generator and two pure WP blocking variable generators. The MA elevated design has $r=A C P q$ as the SP factor generator and $\beta_{1}=A B C$ and $\beta_{2}=A B P$ as the blocking generators.

Again, for scenario $S$ three generators are required. This time, however, one of the blocking variables is formed via separation. The MA design has $r=A B q$ as the 
Figure 9.1: The Three Competing Scenarios, Ilustrating Elevation and Separation

\section{Scenario $D$ $2^{(3+3)-(0+1) \pm(1+0)}$ in}

8 days; 4 rectifiers; 2 blocks

WP factors $A B C$;

SP factors $p q r$

\begin{tabular}{|c|c|}
\multicolumn{1}{|c|}{$\searrow$} \\
Scenario $E$ \\
$2^{(4+2)-(0+1) \pm(2+0)}$ \\
16 days; 2 rectifiers; 4 blocks \\
WP factors $A B C P ;$ \\
SP factors $q r$ \\
\end{tabular}

factor generator and $\beta_{1}=A B C$ and $\delta_{1}=A C p q$ as the blocking generators .

\subsubsection{Comparison of Designs}

Table 9.1 summarizes the advantages and disadvantages of the three designs, with emphasis on main effects and 2fi's only. The presence of at least one asterisk (* or * *) implies that the effect of interest is tested against the WP error for that design. Note that two asterisks appear under Design $D$. This reflects the fact that the WP error in Design $D$ is larger than that in the other two designs, because Design $D$ utilizes only eight days at the WP level. (The variance forms of WP and SP effects for Designs $D, E$ and $S$ are presented in Table 9.2.) The two horizontal lines in 
Table 9.1 are used to set apart the effects, $p, A p, B p$ and $C p$. This is done to remind the reader that under elevation, these are WP effects.

Table 9.1: Precision of Effect Estimates and Alias Structures for the Three Designs

\begin{tabular}{|lccc|}
\hline \hline Effect & Design D & Design E & Design S \\
\hline $\mathrm{A}$ & $* *$ & $*$ & $*$ \\
$\mathrm{~B}$ & $* *$ & $*$ & $*$ \\
$\mathrm{AB}$ & $* *$ & $*$ & $* \mathrm{qr}$ \\
$\mathrm{C}$ & $* *$ & $*$ & $*$ \\
$\mathrm{AC}$ & $* *$ & $*$ & $*$ \\
$\mathrm{BC}$ & $* *$ & $*$ & $*$ \\
\hline $\mathrm{p}$ & & $*$ & \\
$\mathrm{Ap}$ & & $*$ & \\
$\mathrm{Bp}$ & & $*$ & \\
$\mathrm{Cp}$ & & blocks & \\
\hline $\mathrm{q}$ & & & \\
$\mathrm{Aq}$ & & & $\mathrm{Br}$ \\
$\mathrm{Bq}$ & & & $\mathrm{Ar}$ \\
$\mathrm{Cq}$ & & & \\
$\mathrm{pq}$ & & & $*$ \\
$\mathrm{r}$ & & & $\mathrm{Bq}$ \\
$\mathrm{Ar}$ & & & $\mathrm{Aq}$ \\
$\mathrm{Br}$ & & & \\
$\mathrm{Cr}$ & & & \\
$\mathrm{pr}$ & & & $*$ \\
$\mathrm{qr}$ & & $*$ & $*$ \\
\hline
\end{tabular}

Consider Design $D$. On the one hand, it can be seen that all main effects and 2fi's are clear in that they are not aliased with other main effects, 2fi's or blocks. On the other hand, the WP error in design $D$ is larger than that in the other two designs, as noted above and as also seen in Table 9.2.

It would appear that design $E$ is better than design $S$, because there is too much 
Table 9.2: Variances of Estimated Effects for Designs $D, E$ and $S$

\begin{tabular}{|lcc|}
\hline \hline Design & WP Effects & SP Effects \\
\hline$D$ & $\frac{1}{2} \sigma_{w}^{2}+\frac{1}{8} \sigma_{o}^{2}$ & $\frac{1}{8} \sigma_{o}^{2}$ \\
& & \\
$E, S$ & $\frac{1}{4} \sigma_{w}^{2}+\frac{1}{8} \sigma_{o}^{2}$ & $\frac{1}{8} \sigma_{o}^{2}$ \\
\hline
\end{tabular}

aliasing in $S$. It is interesting to note that the experiment that was run was very similar to design $E$. The only difference was that the actual $2^{(4+2)-(0+1) \pm(2+0)}$ experiment used $r=A B C P q$ as the factor generator, rather than $r=A C P q$. Without realizing it at the time we had used the MA design for the unblocked design-and then blocked it-instead of considering all generators at the same time as dictated by the search-table approach. A useful lesson was learned.

\subsubsection{Practical Considerations}

A few practical issues arose in the chrome-plating experiment that are worthy of comment. These deal with center points, blocking and the use of factors that involve concentrations.

Firstly, the actual experiment used 20 days, rather than 16, with the additional days corresponding to center points in the quantitative factors. These points were useful for a number of reasons with the most important of these being that the center points corresponded to current operating conditions.

Secondly, as the experiment proceeded some of the benefits of blocking became evident. For one thing blocking allowed the design to be rescued at one point. This happened on the Monday of the second week, when the bath temperature was supposed to be at its high level. It turned out that, starting from a cold start on Monday morning it was not possible to reach the high temperature when the parts were to be placed in the tank. The design for the third week, which had a low bath temperature on Monday, was interchanged with the design for the second week. A decision was also made to begin heating the bath on Sunday for the third and fourth weeks, in order to reach the high temperature sooner. Another advantage of blocking was that 
interim analyses could be performed at the end of each of the weeks. This could have led to changes in the experimental design, or even to termination, although this did not happen in this experiment.

Thirdly, there was another restriction on randomization that is common in situations involving concentrations. Basically, it is relatively easy to increase the concentration of a solution, but much more difficult to decrease it. This is because decreasing the solution requires that some of the solution be discarded and, in the chrome-plating example, the expense associated with disposal was prohibitive. For this experiment a compromise was reached. It was decided that the bath would be discarded at the end of each week, but that the concentrations (in chrome and sulphate) would increase throughout the week. (Each Wednesday thus corresponded to a center point in the WP factors.) It was recognized that because we did not randomize over the two concentration factors, if something were to change during the week (for example, degradation of the bath) we would have difficulty with inference concerning the concentrations. However, the feeling was that the one week blocks were short enough to prevent such difficulties.

For proprietary reasons, we do not provide analytical results from the chromeplating experiment. One of the approaches that we used, however, involved the construction of two normal probability plots for each response variable - one for the WP effects and one for the SP effects (as determined by the rules in Section 4.2). This analytical approach is typical for FF and FFSP experiments (Daniel, 1959; Box and Jones, 1992; Bingham and Sitter, 2001; Loeppky and Sitter, 2002). The results obtained from the screening experiment enhanced understanding of the chrome-plating process, and led to further experimentation involving the key factors.

Finally, it should be noted that the actual experiment contained two additional factors - plating time and surface preparation. These factors were easier-to-vary than $A, B, C, p, q$ and $r$ and were considered to be sub-subplot factors. By utilizing 4 rectifiers, a $2^{2}$ design in the sub-subplot factors could be performed daily using one part per rectifier for each combination of plating time and surface preparation. However, since the focus in this chapter (and thesis) is on BFFSP designs, we choose to forgo any further discussion pertaining to the sub-subplot factors. 


\section{Chapter 10}

\section{Elevation}

In Chapter 1 the notion of elevation was introduced in context of the chrome-plating experiment. In this case study, we saw that elevation of a SP factor afforded a full, unreplicated $2^{4}$ design at the WP level over the course of the 16 day experiment. This 16-run design at the WP level was viewed as more appealing than an 8-run $2^{3}$ design at the WP level because of the increased precision for the WP effects provided by the 8 additional runs in the $2^{4}$ design. Also, in the chrome-plating experiment elevation provided a means by which less fractionation could be imposed upon the SP level. Recall that the experimenters were originally faced, in essence, with a $2^{3-2}$ design at the SP level due to the availability of only two rectifiers (the SP experimental units) per day in the tank. Instead of running a $1 / 8^{\text {th }}$ fraction at the SP level, elevation of a SP factor implied that a $2^{2-1}$ SP design could be performed daily on the two rectifiers. Thus, by means of elevation, less fractionation was incurred at the SP level. (For an indepth discussion concerning specific design issues in the chrome-plating experiment refer to Chapter 9.)

In contrast to Chapters 1 and 9 this chapter investigates the concept of elevation from a broader perspective. Two elevation approaches are defined and for each approach the impact of elevation on the estimation capacity (aberration, number of clear effects, precision of effects) of selected MA BFFSP designs is documented. It will be shown that elevation is but one approach by which an experimenter may increase the estimation capacity of an existing BFFSP design. Estimation gains may also be realized by means of separation. This chapter investigates potential advantages/disadvantages realized by elevation or separation of selected MA BFFSP de- 
signs. Catalogs of designs are presented detailing cases in which elevation and/or separation may yield superior design choices for an experimenter.

Note that research concerning elevation cannot proceed without satisfaction of one primary assumption - the existence of a versatile factor. A versatile factor is a SP factor, say $p$, that may be elevated to the WP level, say $P$, without causing the experimenter to be unduly concerned with the ensuing loss of precision for the elevated factor as a result of running it at the WP level.

\subsection{Elevation Vs. Separation: As Motivated by the Chrome-Plating Experiment}

In this chapter's introduction we alluded to the fact that there are two approaches to elevating a $2^{\left(n_{1}+n_{2}\right)-\left(k_{1}+k_{2}\right) \pm\left(b_{1}+b_{2}\right)}$ design. The first approach to be evaluated is depicted in Figure 10.1 which is a generalization of the methodology undertaken in the chrome-plating experiment (Figure 9.1). Figure 10.1 depicts the scenario in which an elevated and a separated design are both investigated as possible alternatives to an initial design. One feature of this approach is that the number of WP treatment combinations per block remains constant across the initial, elevated and separated designs. This methodology follows that of the chrome-plating experiment where the initial, elevated and separated designs (Scenarios D, E and S in Figure 9.1) all had four WP treatment combinations per block. The advantage of fixing the number of WP treatments in blocks, across designs, is that the WP variability, $\sigma_{w}^{2}$, can then be thought as constant across designs. That is, fixing the number of WP treatment combinations in a block allows the experimenter to assume that each design possesses the same degree of homogeneity among the WP experimental units. The assumption of constancy of $\sigma_{w}^{2}$ across designs will allow for an accurate comparison of WP effect precision between designs.

A mathematical description of the elevation and separation procedures in Figure 10.1 is now detailed. Firstly, let the set of all possible BFFSP designs be denoted by $\mathcal{D}=\left\{d \mid d=2^{\left(n_{1}+n_{2}\right)-\left(k_{1}+k_{2}\right) \pm\left(b_{1}+b_{2}\right)}\right\}=\left\{d \mid d=\left(n_{1}, n_{2} ; k_{1}, k_{2} ; b_{1}, b_{2}\right)\right\}$. Mappings for both the elevation and separation procedures can then be defined utilizing this notation. For elevation, let $f_{E_{1}}$ be the mapping such that $f_{E_{1}}: \mathcal{D} \longrightarrow \mathcal{D}$, where $f_{E_{1}}(d)=f_{E_{1}}\left(n_{1}, n_{2} ; k_{1}, k_{2} ; b_{1}, b_{2}\right)=\left(n_{1}+1, n_{2}-1 ; k_{1}, k_{2} ; b_{1}+1, b_{2}\right)$. This notation im- 
plies that elevation raises one versatile SP factor, in a $2^{\left(n_{1}+n_{2}\right)-\left(k_{1}+k_{2}\right) \pm\left(b_{1}+b_{2}\right)}$ design, to the WP level such that the resulting design will have one additional WP factor and one fewer SP factor. By increasing the number of WP factors by one, there is a subsequent doubling of the number of WP treatment combinations in the elevated design. To ensure that the number of WP treatment combinations per block is equal to that of the initial design, one additional pure WP blocking variable is required in the elevated design. Therefore, $b_{1}$ is increased by 1 as depicted in the bottom left hand box of Figure 10.1.

Figure 10.1: Elevation Vs. Separation: As Motivated by the Chrome-Plating Experiment.

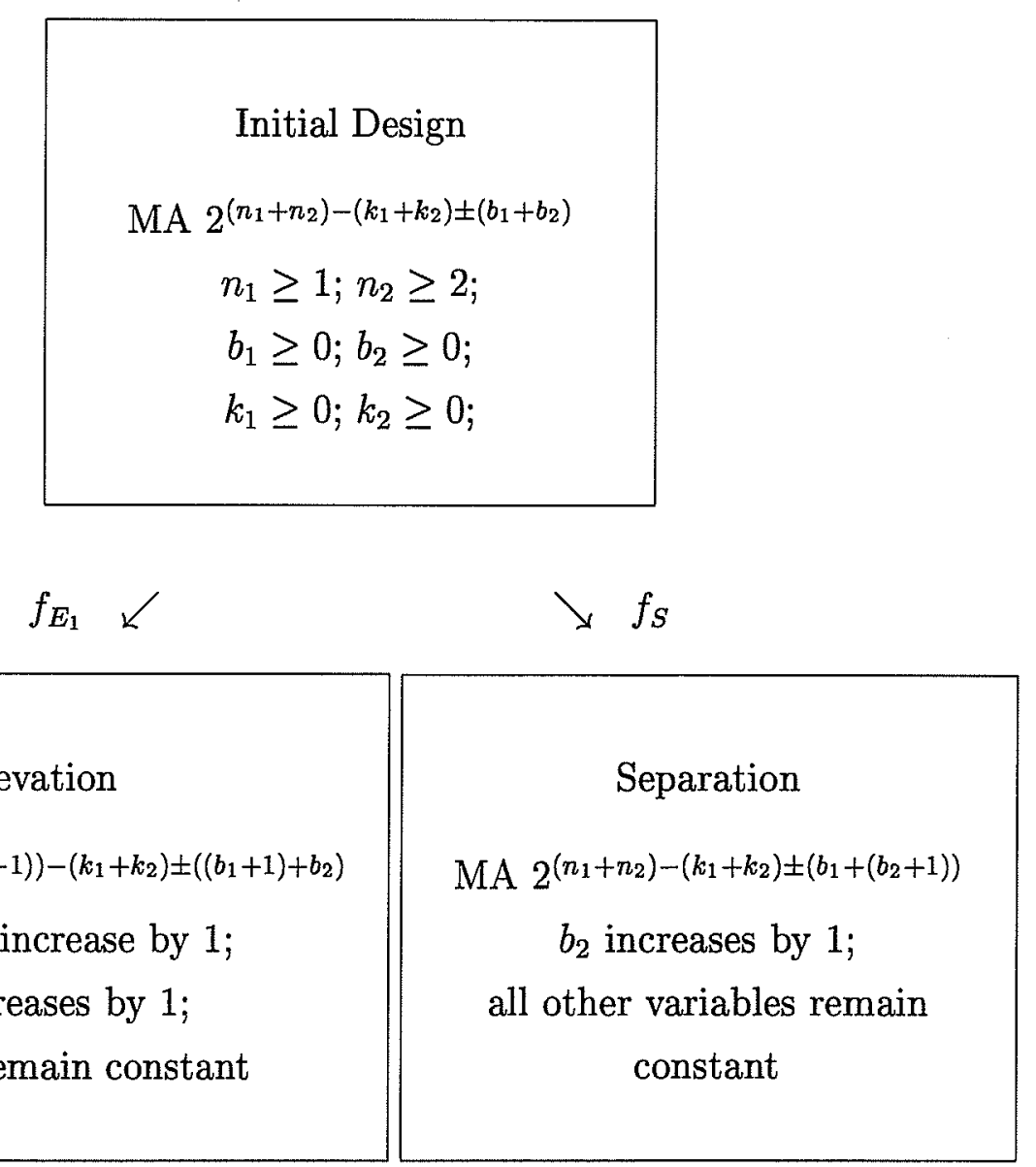

Let $f_{S}$ symbolize the mapping which occurs under separation. Here, $f_{S}: \mathcal{D} \longrightarrow \mathcal{D}$, where $f_{S}(d)=f_{S}\left(n_{1}, n_{2} ; k_{1}, k_{2} ; b_{1}, b_{2}\right)=\left(n_{1}, n_{2} ; k_{1}, k_{2} ; b_{1}, b_{2}+1\right)$. Under separation, no SP factor is elevated; rather, pseudo-replication of the WP treatment combina- 
tions is achieved with the presence of an additional separator (Chapter 3). (Note that $b_{2}$ is increased by 1 as illustrated in the bottom right hand box of Figure 10.1.) The additional separator causes each WP treatment level setting to be reset twice as often, hence the replication. Also, observe that the presence of the additional separator implies that there will be twice as many blocks, $2^{b_{1}+\left(b_{2}+1\right)}$, as compared to the initial design. This doubling of the number of blocks ensures that the number of WP treatment combinations per block is equal to that of the initial design. Furthermore, from the comments in the previous paragraph we may infer that both the elevated and separated designs have an equal number of WP treatment combinations per block.

It can be seen that the initial, elevated and separated designs in Figure 10.1 are fundamentally the "same". By this we mean that from a practitioner's perspective each design could be run just as easily as another. To see this, consider the following. Firstly, all three designs require the same number of runs. Therefore, the initial, elevated and separated designs all require the same number of experimental units and intuitively they should then require an identical amount of resources (time, money, personnel) to complete.

Secondly, the presence of an additional WP factor in the elevated design will not increase the complexity of the experiment. Note that the elevated factor is inherently an easy-to-vary factor, evidenced by its placement at the SP level in the initial design. Therefore, raising this easy-to-vary factor should not impose any additional strain upon the resetting of the other WP treatment levels.

Thirdly, as was mentioned previously, the restriction that the number of WP treatments per block be fixed across the three designs enables a "fair" comparison of $\sigma_{w}^{2}$, the WP variability. This constancy of WP variability across the designs prevents "apple and orange" comparisons in terms of precision of estimated WP effects. This point will be expounded upon in subsequent sections.

\subsubsection{Some Comments and an Example}

Before proceeding to an example some additional general observations regarding the implications of Figure 10.1 are in order. Firstly, elevation always requires that an initial design possess $n_{1} \geq 1$ and $n_{2} \geq 2$. If the initial design has $n_{2}=1$, this implies that the elevated design has $n_{2}=0$ which in turn implies that the elevated design 
is not a split-plot design. In this case, the elevated design could be thought of as a two-level BFF design, a scenario not of interest in this research.

Secondly, it has been noted that elevation of one SP factor or the presence of one additional separator doubles the number of runs at the WP level. This increase in WP runs serves to increase the precision of the WP effect estimates, a result developed first in Chapter 4 in the context of separation and in Chapter 9 in the context of the elevated chrome-plating design. The important point here is that the elevation and separation procedures yield an identical increase in precision for the estimation of WP effects. More will be said about this point after the following example.

Example 10.1.1 Suppose experimenters in a manufacturing company initially contemplate running a $2^{(3+5)-(1+2) \pm(0+1)}$ design. The 32-run design, perhaps too large to be completed in one shift, is to be run in two blocks. After careful consideration of the design, suppose that the experimenters are not satisfied with the degree of precision at the WP level that this design provides. An effort should then be made to explore methods by which the precision of the yet-to-be estimated WP effects may be increased.

Note that the $2^{(3+5)-(1+2) \pm(0+1)}$ design already includes one separator $\left(b_{2}=1\right)$ but assume that the resulting 8 WP runs are not sufficient for achieving a satisfactory level of precision for the WP effects. In this setting, consideration of a design incorporating an elevated factor, or perhaps an additional separator may lead to a better design choice. Following the general approach outlined in Figure 10.1, the two design alternatives are the $2^{(4+4)-(1+2) \pm(1+1)}$ elevated design (Design $A$ ) and the $2^{(3+5)-(1+2) \pm(0+2)}$ design (Design $\left.B\right)$-note the additional separator in the latter design. All three designs have four WP factor combinations per block so accurate comparisons of WP errors across designs can be made.

Table 10.1 assists in summarizing the advantages and/or disadvantages of the three designs in Example 10.1.1. Specifically, Table 10.1 provides the following summary:

1. Lists all factor main effects and 2fi's for each design.

2. Differentiates between clear and unclear treatment effects.

3. Provides the alias chain for each unclear treatment effect. 
Table 10.1: Precision of Effect Estimates and Alias Structures for the Initial, Elevated and Separated Designs in Example 10.1.1.

\begin{tabular}{|c|c|c|c|}
\hline Effect & Initial Design & Design A & Design $B$ \\
\hline $\mathbf{A}$ & $--B C$ & - & $-\mathrm{BC}$ \\
\hline B & $--A C$ & - & $-A C$ \\
\hline $\mathrm{AB}$ & $--\mathrm{C}$ & -CD, blocks & $-\mathrm{C}$ \\
\hline $\mathrm{C}$ & $--A B$ & - & $-A B$ \\
\hline $\mathrm{AC}$ & $--\mathrm{B}$ & $-B D, q r$ & $-\mathrm{B}$ \\
\hline $\mathrm{BC}$ & $--\mathrm{A}$ & $-A D, p s$ & $-A$ \\
\hline$D \backslash t$ & & - & - \\
\hline$A D \backslash A t$ & $\mathrm{rs}$ & $-\mathrm{BC}, \mathrm{ps}$ & blocks \\
\hline $\mathrm{BD} \backslash \mathrm{Bt}$ & $\mathrm{pq}$ & $-\mathrm{AC}, \mathrm{qr}$ & - \\
\hline $\begin{array}{l}\mathrm{CD} \backslash \mathrm{Ct} \\
\mathrm{p}\end{array}$ & & $-\mathrm{AB}$, blocks & $-p q, r s$ \\
\hline Ap & & Ds & \\
\hline Bp & $\mathrm{qt}$ & Cs & \\
\hline $\mathrm{Cp}$ & & Bs & qt \\
\hline $\begin{array}{l}\mathrm{Dp} \backslash \mathbf{p t} \\
\mathrm{q}\end{array}$ & $\mathrm{Bq}$ & As & $\mathrm{Cq}$ \\
\hline Aq & & $\mathrm{Cr}$ & \\
\hline $\mathrm{Bq}$ & $\mathrm{pt}$ & Dr & \\
\hline $\mathrm{Cq}$ & & $\mathrm{Ar}$ & pt \\
\hline $\begin{array}{l}\mathbf{D q} \backslash \mathbf{q t} \\
\mathbf{r}\end{array}$ & Bp & $\mathrm{Br}$ & $\mathrm{Cp}$ \\
\hline Ar & st & $\mathrm{Cq}$ & \\
\hline $\mathrm{Br}$ & & $\mathrm{Dq}$ & \\
\hline $\mathrm{Cr}$ & & $\mathrm{Aq}$ & st \\
\hline $\begin{array}{l}\mathrm{Dr} \backslash \mathbf{r t} \\
\mathrm{s}\end{array}$ & As & $\mathrm{Bq}$ & Cs \\
\hline As & $\mathrm{rt}$ & $\mathrm{Dp}$ & \\
\hline Bs & & Cp & \\
\hline
\end{tabular}


Table 10.1 (Cont'd)

\begin{tabular}{||llll||}
\hline Effect & Initial Design & Design A & Design B \\
\hline Cs & & $\mathrm{Bp}$ & $\mathrm{rt}$ \\
Ds $\backslash$ st & $\mathrm{Ar}$ & $\mathrm{Ap}$ & $\mathrm{Cr}$ \\
pq & $-\mathrm{Bt}$ & - & $-\mathrm{rs}, \mathrm{Ct}$ \\
pr & -- & - & $-\mathrm{qs}$ \\
ps & & $-\mathrm{AD}, \mathrm{BD}$ & $-\mathrm{qr}$ \\
qr & & $-\mathrm{AC}, \mathrm{BD}$ & $-\mathrm{ps}$ \\
qs & -- & - & $-\mathrm{pr}$ \\
rs & $\mathrm{At}$ & - & $-\mathrm{pq}, \mathrm{Ct}$ \\
\hline
\end{tabular}

4. Distinguishes between those effects that are tested against the WP and SP errors.

5. Gives indication to the size of a design's WP error.

Note that to correctly read Table 10.1 one must replace $t$ with $D$ (and vice versa) within the appropriate effects when moving between the initial and elevated designs. (That is, we are explicitly assuming that treatment factor $t$ is the versatile SP factor in the $2^{(3+5)-(1+2) \pm(0+1)}$ design.) Using Table 10.1 the following observations are noted:

1. The initial design has less aliasing of SP main effects and 2fi's. In fact, the initial design has 13 clear SP 2fi's whereas the elevated and separated designs have only 4 and 9 , respectively.

2. Effects tested against WP error are indicated by either one dash (-) or two dashes (- -). The presence of two dashes reflects a larger WP error for the corresponding main effect or $2 \mathrm{f}$. The elevated and separated designs both have smaller WP errors (that is, greater precision) than the initial design; therefore, their WP errors are denoted by only a single dash.

3. The $2^{(4+4)-(1+2) \pm(1+1)}$ elevated design has the "best" alias structure at the WP level. In this design, all WP main effects are clear. All WP main effects are unclear in the initial and separated designs. 
4. Overall, the elevated design has the worst alias structure in that it possesses only 12 clear WP and SP main effects and 2fi's. The initial and separated designs have 18 and 14 clear WP and SP main effects and 2f's, respectively.

\subsubsection{The Initial, Elevated and Separated BFFSP Design Catalog}

In this section we outline the construction and use of the BFFSP design catalog, Appendix $\mathrm{C}$, containing MA initial, elevated and separated BFFSP designs. For a given initial MA $2^{\left(n_{1}+n_{2}\right)-\left(k_{1}+k_{2}\right) \pm\left(b_{1}+b_{2}\right)}$ design, the catalog provides a corresponding MA $2^{\left(\left(n_{1}+1\right)+\left(n_{2}-1\right)\right)-\left(k_{1}+k_{2}\right) \pm\left(\left(b_{1}+1\right)+b_{2}\right)}$ elevated design and a MA $2^{\left(n_{1}+n_{2}\right)-\left(k_{1}+k_{2}\right) \pm\left(b_{1}+\left(b_{2}+1\right)\right)}$ separated design. Each group of three associated designs is referred to as a "triple", where the elevated and separated designs represent alternative choices available to an experimenter in light of an initial design.

For brevity, the initial designs that we will consider represent only a subset of the 32-run MA BFFSP designs already tabled in Appendix B. Also, we will not investigate the 8 and 16-run design scenarios here. It is envisioned that this catalog of triples contains a large number of the 32-run BFFSP design possibilities facing an experimenter and thereby is considered an extensive although not an exhaustive listing. Each initial MA design in a triple must possess $n_{1} \geq 1$ and $n_{2} \geq 2$. These restrictions are a consequence of the discussion in Section 10.1.1, where we explained that if these inequalities do not hold a split-plot design will not be formed by our elevation procedure.

When selecting an initial MA $2^{\left(n_{1}+n_{2}\right)-\left(k_{1}+k_{2}\right) \pm\left(b_{1}+b_{2}\right)}$ design from Appendix B, to use in Appendix $\mathrm{C}$, we take the MA design having the highest ranked estimation capacity - if there are multiple MA designs listed for a specific value of $n_{1}, n_{2} ; k_{1}, k_{2} ; b_{1}, b_{2}$.

Appendix $\mathrm{C}$ contains separate tables of triples for each of the following scenarios:

1. The initial design is a MA $2^{\left(n_{1}+n_{2}\right)-\left(k_{1}+k_{2}\right)}$ FFSP design.

2. The initial design is a MA $2^{\left(n_{1}+n_{2}\right)-\left(k_{1}+k_{2}\right) \pm\left(b_{1}+0\right)}$ BFFSP design with pure WP blocking.

3. The initial design is a MA $2^{\left(n_{1}+n_{2}\right)-\left(k_{1}+k_{2}\right) \pm\left(0+b_{2}\right)}$ BFFSP design with separation. 
4. The initial design is a MA $2^{\left(n_{1}+n_{2}\right)-\left(k_{1}+k_{2}\right) \pm\left(b_{1}+b_{2}\right)}$ BFFSP design with mixed blocking.

All designs have between six and ten factors and blocking variables (combined) and consist of 32 runs in either two, four or eight blocks. Each $2^{\left(n_{1}+n_{2}\right)-\left(k_{1}+k_{2}\right) \pm\left(b_{1}+b_{2}\right)}$ BFFSP design is abbreviated "Design $=n_{1}, n_{2} ; k_{1}, k_{2} ; b_{1}, b_{2}$ ". Within the tables the designs are presented in ascending order of $n=n_{1}+n_{2}+b_{1}+b_{2}$, the total number of factors and blocking variables in the initial design. As in the MA BFFSP design catalog, the WLPs are truncated at the last non-zero value. A-G and p-w are used to denote the WP and SP factors, respectively, with the last letter in each generator representing the added factor. The $i^{\text {th }}$ and $j^{\text {th }}$ WP blocking variable and separator are denoted by $\beta_{i}$ and $\delta_{j}$. In columns (a) - (f) we have again included the assessment of each BFFSP design with respect to the six optimality criteria of Section 5.3.

One distinguishing feature of this catalog is that, within a design triple, each design has the same number of WP treatment combinations per block. As previously discussed, this design characteristic provides the user with the ability to accurately compare WP errors across associated designs.

The following example provides the reader with an introduction to the use of the "initial, elevated and separated BFFSP design catalog".

Example 10.1.2 In Example 10.1.1 we envisioned an experimenter choosing between the $2^{(3+5)-(1+2) \pm(0+1)}, 2^{(4+4)-(1+2) \pm(1+1)}$ and $2^{(3+5)-(1+2) \pm(0+2)}$ designs. In that example we provided Table 10.1 to assist in the selection process. However, in most cases a table of such detail is not necessary. Rather, the practitioner should refer to Appendix $C$ to quickly determine whether or not elevation and/or separation provide designs with greater estimation capacity than that of the initial design.

To find the initial, elevated and separated designs of Example 10.1.1, scan Table C.3 (in Appendix C) until you reach those designs having $n=n_{1}+n_{2}+b_{1}+b_{2}=9$. In this catalog $n$ reflects the number of variables in the initial design. (Note that the elevated and separated designs will always have a value of $n$ one greater than that of the initial design. This is due to the presence of the additional blocking variable in these designs.) After locating the correct triple (denoted by 3,5;1,2;0,1 and $4,4 ; 1,2 ; 1,1$ and 3,$5 ; 1,2 ; 0,2)$ one can make the necessary comparisons. As detailed in Example 10.1.1, the initial design outperforms the elevated and separated designs in terms of the total number of clear main effects and 2fi's (criteria (a) and (b)). 
However, the elevated design is "optimal" with respect to the number of clear WP main effects (4). Neither the initial or separated designs have any clear WP main effects.

What is not obvious from criteria (a) - ( $f$ ) in the catalog is that the elevated and separated designs possess a smaller WP error than the initial design. These precision gains are, to a large extent, the motivation behind considering the elevated and separated designs in the first place. The exact form of the WP error in elevated and separated BFFSP designs is provided in Section 10.1.3.

Again, one should be aware that not every possible 32-run design triple is present within the catalog. We have chosen to include only those triples in which the elevated design is superior in at least one of the criteria (a) - (f), compared to the separated design. To include elevated designs which are inferior in all six of the criteria would only serve to lengthen the catalog. As a result, some triples that one might expect to be present in Appendix $\mathrm{C}$ have been completely omitted. The implication of these omissions is that, for the given 32-run initial design, no elevated design could be constructed that was superior to the corresponding separated design in any of the criteria, (a) - (f).

Finally, one may have observed the presence of horizontal lines within the tables while working through Example 10.1.1. The horizontal lines are used to distinguish between triples having identical values for $n=n_{1}+n_{2}+b_{1}+b_{2}$.

\subsubsection{Additional Comments}

In this section we formalize several of the implications of the elevation and separation procedures, as outlined in Figure 10.1.

Firstly, elevation causes twice as many distinct WP treatment combinations to be run as there would have been otherwise. Separation provides a pseudo-replication of existing WP treatment combinations. Therefore, elevation and separation yield small(er) variances for effects at the WP level of the design. This result can be seen by considering the general variance form of a WP effect, $\operatorname{Var}(A)=\frac{4}{N}\left(2^{n_{2}-k_{2}-b_{2}} \sigma_{w}^{2}+\sigma_{o}^{2}\right)$, where $A$ is a given WP factor (Chapter 4). We observe from this variance structure that if a SP factor is elevated, then the exponent $n_{2}-k_{2}-b_{2}$ will decrease by 1 , due to the unit reduction in $n_{2}$. Also, if separation occurs, $n_{2}-k_{2}-b_{2}$ will again decrease 
by 1 , due to a unit increase in $b_{2}$. Either approach produces a smaller numerator in the coefficient of $\sigma_{w}^{2}$, thus decreasing the size of the WP error.

Secondly, we anticipate that the overall alias structure of elevated and separated designs will, in general, be worse than that of the corresponding initial design. This observation arises from the fact that the elevated and separated designs require one additional blocking variable than the initial design. This additional variable has the effect of increasing the degree of aliasing within the DCSs of the elevated and separated designs. Anticipated consequences of greater aliasing among factor effects include fewer clear SP main effects and 2fi's. A scenario evidencing this possibility was given in Example 10.1.1.

Thirdly, despite the fact that the overall alias structure of an elevated design may be worse than that of the initial design, one may actually observe a decrease in aliasing at the WP level. This possibility (of which Example 10.1.1 is a case in point) arises due to the presence of the additional WP factor in elevated designs. Therefore, if interest in the WP factors is high, elevation may provide a means for higher estimation capacity at the WP level of a BFFSP design.

\subsection{Elevation: Another Approach}

The introduction of this chapter mentioned that two approaches to elevation were to be considered. The distinguishing feature of the elevation procedure described in Section 10.1 is that each (initial, elevated and separated) design in a triple had equal numbers of WP units per block. This condition was imposed so as to maintain a fair comparability of $\sigma_{w}^{2}$ across the designs. If one relaxes this condition another elevation procedure can be described, as outlined in Figure 10.2.

There are two primary differences between Figures 10.1 and 10.2. Firstly, the approach outlined in Figure 10.2 does not consider design triples but rather pairs of designs only. That is, in this section there is no direct comparison of separation with elevation. The decision to only compare initial and elevated design pairs is driven by practicality (size of design catalog) rather than by theory.

Secondly, the elevation approach of Figure 10.2 considers only those design pairs in which the number of WP units per block differs between the initial and elevated designs. Conditions are stipulated in Figure 10.2 that allow these differences to occur. 
Figure 10.2: Elevation: Another Approach

\begin{tabular}{c} 
Initial Design \\
MA $2^{\left(n_{1}+n_{2}\right)-\left(k_{1}+k_{2}\right) \pm\left(b_{1}+b_{2}\right)}$ \\
$n_{1} \geq 1 ; n_{2} \geq 2$ \\
$b_{1} \geq 0 ; b_{2} \geq 0$ \\
$k_{1} \geq 0 ; k_{2} \geq 0$ \\
\hline
\end{tabular}

$$
f_{E_{2}} \downarrow
$$

\section{Elevated Design}

MA $2^{\left(\left(n_{1}+1\right)+\left(n_{2}-1\right)\right)-\left(k_{1}+k_{2}\right) \pm\left(b_{1}^{\prime}+b_{2}^{\prime}\right)}$

$n_{1}$ increases by $1 ; n_{2}$ decreases by 1 ; $b_{1}^{\prime}+b_{2}^{\prime}=b_{1}+b_{2}$, so $b_{1}^{\prime}, b_{2}^{\prime}$ either both vary or both stay the same

In defense of holding $b_{1}+b_{2}$ fixed across design pairs we present the following two arguments:

1. Changing the total number of blocks from a proposed (initial) design may impede, from a practical standpoint, completion of the experiment. For example, suppose a 32-run BFFSP experiment is desired. If only two shifts (blocks) of workers are available for the experiment, an increase in $b_{1}+b_{2}$ between the initial and elevated designs may be infeasible from a personnel perspective.

2. Although $\sigma_{w}^{2}$ will probably differ across designs if the number of WPs per block differs across designs, this difference could be small if the difference in the number of WPs per block is small. For example, if the initial design has 4 WPs per block and the elevated design has 8 WPs per block, the dissimilarities between the designs' $\sigma_{w}^{2}$ s may be small if WPs tend to be homogenous with 
one another.

The elevation procedure of Figure 10.2 may be described as follows. As in Section 10.1, let the set of all possible BFFSP designs be denoted by $\mathcal{D}=\{d \mid d=$ $\left.2^{\left(n_{1}+n_{2}\right)-\left(k_{1}+k_{2}\right) \pm\left(b_{1}+b_{2}\right)}\right\}=\left\{d \mid d=\left(n_{1}, n_{2} ; k_{1}, k_{2} ; b_{1}, b_{2}\right)\right\}$. Let $f_{E_{2}}$ be the mapping such that $f_{E_{2}}: \mathcal{D} \longrightarrow \mathcal{D}$, where $f_{E_{2}}(d)=f_{E_{2}}\left(n_{1}, n_{2} ; k_{1}, k_{2} ; b_{1}, b_{2}\right)=\left(n_{1}+1, n_{2}-\right.$ $\left.1 ; k_{1}, k_{2} ; b_{1}^{\prime}, b_{2}^{\prime}\right)$. This notation implies that elevation raises one versatile SP factor, in a $2^{\left(n_{1}+n_{2}\right)-\left(k_{1}+k_{2}\right) \pm\left(b_{1}+b_{2}\right)}$ design, to the WP level such that the resulting design will have one additional WP factor and one fewer SP factor. By increasing the number of WP factors by one, there is a subsequent doubling of the number of WP treatment combinations in the elevated design. Since $f_{E_{2}}$ causes twice as many WP treatment combinations to be formed there is an increase in precision at the WP level. This result parallels that for the mapping $f_{E_{1}}$. However, unlike $f_{E_{1}}, f_{E_{2}}$ does not produce elevated designs with the same number of WP units per block as the initial design. Therefore, $b_{1}$ is not necessarily increased by 1 under this elevation approach.

The following example illustrates one scenario where elevation, via $f_{E_{2}}$, provides a design with appealing estimation qualities.

Example 10.2.1 Consider the resolution 3, $M A 2^{(5+3)-(2+1) \pm(1+0)}$ design (Appendix B). This design has 3 clear SP main effects and 18 clear SP 2fi's. However, no WP main effects or 2fi's are clear. This lack of estimation ability at the WP level may be unattractive to an experimenter if some information regarding WP effects is desired. Elevation of this design to a MA $2^{(6+2)-(2+1) \pm(1+0)}$ design allows all 6 WP main effects to be clearly estimated. In addition, there is increased precision for estimating the variance of these main effects due to the increased number of WP runs. More precisely, under elevation the variance of a WP effect decreases from $\frac{1}{2} \sigma_{w}^{2}+\frac{1}{8} \sigma_{o}^{2}$ to $\frac{1}{4} \sigma_{w}^{2}+\frac{1}{8} \sigma_{o}^{2}$ (see Section 10.1.3 for the general variance form of a WP effect).

The primary drawback of elevation in this scenario is that one fewer SP main effect and five fewer SP 2fi's are estimable in the $2^{(6+2)-(2+1) \pm(1+0)}$ design. When elevation is performed this sacrifice in terms of the total number of clear SP main effects and 2fi's is often observed. Specifically, in the elevated design it should be anticipated that there will be one fewer clear SP main effect (due to elevation of the SP factor). In addition, all 2fi's involving the versatile SP factor and other WP factors in the initial design will "disappear" due to elevation. 


\subsubsection{The Initial and Elevated BFFSP Design Catalog}

In this section we outline the construction and use of the BFFSP design catalog, Appendix D, containing MA initial and elevated BFFSP design pairs. For an initial MA $2^{\left(n_{1}+n_{2}\right)-\left(k_{1}+k_{2}\right) \pm\left(b_{1}+b_{2}\right)}$ design, the catalog provides a corresponding MA $2^{\left(\left(n_{1}+1\right)+\left(n_{2}-1\right)\right)-\left(k_{1}+k_{2}\right) \pm\left(b_{1}^{\prime}+b_{2}^{\prime}\right)}$ elevated design as implied by the mapping $f_{E_{2}}$. The elevated design in a pair represents an alternative to the initial design. Therefore, this catalog provides one method by which a practitioner may choose a design that best satisfies the estimation demands for a particular BFFSP experiment.

Appendix D contains only those 32-run initial and elevated design pairs in which the elevated design is superior in at least one of the criteria (a) - (f). To include elevated designs which are inferior in all six of the criteria would unnecessarily lengthen the catalog. As a result, some pairs that one might expect to be present in Appendix $\mathrm{D}$ have been completely omitted. The implication of these omissions is that, for the given 32-run initial design, no elevated design could be constructed that was superior in any of the criteria, (a) - (f).

When selecting an initial MA $2^{\left(n_{1}+n_{2}\right)-\left(k_{1}+k_{2}\right) \pm\left(b_{1}+b_{2}\right)}$ design from Appendix B, to use in Appendix D, we take the MA design having the highest ranked estimation capacity - if there are multiple MA designs listed for a specific value of $n_{1}, n_{2} ; k_{1}, k_{2} ; b_{1}, b_{2}$.

For the mapping defined by $f_{E_{2}}$, the following list enumerates all possible elevation scenarios for 32-run BFFSP designs:

1. Elevation of a BFFSP design with pure WP blocking to another BFFSP design with pure WP blocking,

2. Elevation of a separated BFFSP design to another separated BFFSP design,

3. Elevation of a separated BFFSP design to a BFFSP design with pure WP blocking,

4. Elevation of a BFFSP design with pure WP blocking to a separated BFFSP design.

All designs have between seven and ten factors and blocking variables (combined) and consist of 32 runs in either two or four blocks. Each $2^{\left(n_{1}+n_{2}\right)-\left(k_{1}+k_{2}\right) \pm\left(b_{1}+b_{2}\right)}$ BFFSP design is abbreviated "Design $=n_{1}, n_{2} ; k_{1}, k_{2} ; b_{1}, b_{2}$ ". Within the tables the designs 
are presented in ascending order of $n=n_{1}+n_{2}+b_{1}+b_{2}$, the total number of factors and blocking variables. As in the MA BFFSP design catalog, the WLPs are truncated at the last non-zero value. A-G and p-w are used to denote the WP and SP factors, respectively, with the last letter in each generator representing the added factor. The $i^{\text {th }}$ and $j^{\text {th }}$ WP blocking variable and separator are denoted by $\beta_{i}$ and $\delta_{j}$. In columns (a) - (f) we have again included the assessment of each BFFSP design with respect to the six optimality criteria of Section 5.3.

The following example provides the reader with an introduction to the use of the initial and elevated BFFSP design catalog.

Example 10.2.2 Consider again the $2^{(5+3)-(2+1) \pm(1+0)}$ and $2^{(6+2)-(2+1) \pm(1+0)}$ designs in Example 10.2.1. This initial and elevated design pair may be found in Table D.1 in Appendix D. The values for criteria (a) - (f) are listed for both designs. Again, the elevated $2^{(6+2)-(2+1) \pm(1+0)}$ design provides the experimenter with a superior design choice if there is significant interest in the WP main effects (refer to the discussion in Example 10.2.1).

What is not directly obvious from Appendix $D$ is that an elevated design possesses a smaller WP error than its corresponding initial design. These precision gains should not be ignored since they are, to a large extent, the motivation behind considering elevation in the first place. The general form of the WP error in elevated designs is provided in Section 10.1.3.

\subsubsection{Additional Comments}

This section highlights the primary advantages/disadvantages of elevation via $f_{E_{2}}$. The key advantages are:

1. Precision gains at the WP level: Elevation of a versatile SP factor causes twice as many distinct WP treatment combinations to be run. The consequence of the additional runs on the form of the variance for a WP factor is the same as that for elevation under the mapping, $f_{E_{1}}$.

2. Decreased aliasing at the WP level: The additional WP factor may produce WP generators with greater word lengths. Consequently, more WP effects may be clear in the elevated design. 
3. Consistency in the number of blocks between initial and elevated designs: The advantage of fixed blocks is more practical than statistical. For example, if the number of blocks (work shifts, batches of material) cannot be practically changed, elevation under $f_{E_{2}}$ may be reasonable.

The key disadvantages are:

1. Possible dissimilarities in $\sigma_{w}^{2}$ between the initial and elevated designs: Unless the WPs are reasonably homogeneous, the WP errors of initial and elevated designs may be incongruous.

2. Elevation of a WP factor typically results in fewer clear SP main effects and 2 fi's. 
Appendix A

Standard Run Order of Selected FFSP and BFFSP Designs 
Table A.1: Standard Run Order of the $2^{(3+3)-(0+1)}$ FFSP Design.

\begin{tabular}{|c|c|c|c|c|c|c|c|}
\hline Run & A & B & $\mathrm{C}$ & $\mathrm{p}$ & $q$ & $\mathrm{r}$ & Error Term \\
\hline 1 & - & - & - & - & - & - & $e_{1}+\epsilon_{1(1)}$ \\
\hline 2 & & & & + & - & + & $e_{1}+\epsilon_{2(1)}$ \\
\hline 3 & & & & - & + & + & $e_{1}+\epsilon_{3(1)}$ \\
\hline 4 & & & & + & + & - & $e_{1}+\epsilon_{4(1)}$ \\
\hline 5 & + & - & - & - & - & + & $e_{2}+\epsilon_{1(2)}$ \\
\hline 6 & & & & + & - & - & $e_{2}+\epsilon_{2(2)}$ \\
\hline 7 & & & & - & + & - & $e_{2}+\epsilon_{3(2)}$ \\
\hline 8 & & & & + & + & + & $e_{2}+\epsilon_{4(2)}$ \\
\hline 9 & - & + & - & - & - & + & $e_{3}+\epsilon_{1(3)}$ \\
\hline 10 & & & & + & - & - & $e_{3}+\epsilon_{2(3)}$ \\
\hline 11 & & & & - & + & - & $e_{3}+\epsilon_{3(3)}$ \\
\hline 12 & & & & + & + & + & $e_{3}+\epsilon_{4(3)}$ \\
\hline 13 & + & + & - & - & - & - & $e_{4}+\epsilon_{1(4)}$ \\
\hline 14 & & & & + & - & + & $e_{4}+\epsilon_{2(4)}$ \\
\hline 15 & & & & - & + & + & $e_{4}+\epsilon_{3(4)}$ \\
\hline 16 & & & & + & + & - & $e_{4}+\epsilon_{4(4)}$ \\
\hline 17 & - & - & + & - & - & + & $e_{5}+\epsilon_{1(5)}$ \\
\hline 18 & & & & + & - & - & $e_{5}+\epsilon_{2(5)}$ \\
\hline 19 & & & & - & + & - & $e_{5}+\epsilon_{3(5)}$ \\
\hline 20 & & & & + & + & + & $e_{5}+\epsilon_{4(5)}$ \\
\hline 21 & + & - & + & - & - & - & $e_{6}+\epsilon_{1(6)}$ \\
\hline 22 & & & & + & - & + & $e_{6}+\epsilon_{2(6)}$ \\
\hline 23 & & & & - & + & + & $e_{6}+\epsilon_{3(6)}$ \\
\hline 24 & & & & + & + & - & $e_{6}+\epsilon_{4(6)}$ \\
\hline 25 & - & + & + & - & - & - & $e_{7}+\epsilon_{1(7)}$ \\
\hline 26 & & & & + & - & + & $e_{7}+\epsilon_{2(7)}$ \\
\hline 27 & & & & - & + & + & $e_{7}+\epsilon_{3(7)}$ \\
\hline 28 & & & & + & + & - & $e_{7}+\epsilon_{4(7)}$ \\
\hline
\end{tabular}


Table A.1 (Cont'd)

\begin{tabular}{||cccccccc||}
\hline Run & A & B & C & p & q & r & Error Term \\
\hline 29 & + & + & + & - & - & + & $e_{8}+\epsilon_{1(8)}$ \\
30 & & & & + & - & - & $e_{8}+\epsilon_{2(8)}$ \\
31 & & & & - & + & - & $e_{8}+\epsilon_{3(8)}$ \\
32 & & & & + & + & + & $e_{8}+\epsilon_{4(8)}$ \\
\hline
\end{tabular}


Table A.2: Standard Run Order of the $2^{(3+3)-(0+1) \pm(1+0)}$ BFFSP Design.

\begin{tabular}{|c|c|c|c|c|c|c|c|c|c|}
\hline Run & A & B & C & $\mathrm{p}$ & $q$ & $\mathrm{r}$ & $\beta_{1}$ & Block & Error Term \\
\hline 1 & - & - & - & - & - & + & - & 1 & $e_{1(1)}+\epsilon_{1(11)}$ \\
\hline 2 & & & & + & - & - & - & 1 & $e_{1(1)}+\epsilon_{2(11)}$ \\
\hline 3 & & & & - & + & - & - & 1 & $e_{1(1)}+\epsilon_{3(11)}$ \\
\hline 4 & & & & + & + & + & - & 1 & $e_{1(1)}+\epsilon_{4(11)}$ \\
\hline 5 & + & + & - & - & - & + & - & 1 & $e_{2(1)}+\epsilon_{1(12)}$ \\
\hline 6 & & & & + & - & - & - & 1 & $e_{2(1)}+\epsilon_{2(12)}$ \\
\hline 7 & & & & - & + & - & - & 1 & $e_{2(1)}+\epsilon_{3(12)}$ \\
\hline 8 & & & & + & + & + & - & 1 & $e_{2(1)}+\epsilon_{4(12)}$ \\
\hline 9 & + & - & + & - & - & - & - & 1 & $e_{3(1)}+\epsilon_{1(13)}$ \\
\hline 10 & & & & + & - & + & - & 1 & $e_{3(1)}+\epsilon_{2(13)}$ \\
\hline 11 & & & & - & + & + & - & 1 & $e_{3(1)}+\epsilon_{3(13)}$ \\
\hline 12 & & & & + & + & - & - & 1 & $e_{3(1)}+\epsilon_{4(13)}$ \\
\hline 13 & - & + & + & - & - & - & - & 1 & $e_{4(1)}+\epsilon_{1(14)}$ \\
\hline 14 & & & & + & - & + & - & 1 & $e_{4(1)}+\epsilon_{2(14)}$ \\
\hline 15 & & & & - & + & + & - & 1 & $e_{4(1)}+\epsilon_{3(14)}$ \\
\hline 16 & & & & + & + & - & - & 1 & $e_{4(1)}+\epsilon_{4(14)}$ \\
\hline 17 & + & - & - & - & - & - & + & 2 & $e_{1(2)}+\epsilon_{1(21)}$ \\
\hline 18 & & & & + & - & + & + & 2 & $e_{1(2)}+\epsilon_{2(21)}$ \\
\hline 19 & & & & - & + & + & + & 2 & $e_{1(2)}+\epsilon_{3(21)}$ \\
\hline 20 & & & & + & + & - & + & 2 & $e_{1(2)}+\epsilon_{4(21)}$ \\
\hline 21 & - & + & - & - & - & - & + & 2 & $e_{2(2)}+\epsilon_{1(22)}$ \\
\hline 22 & & & & + & - & + & + & 2 & $e_{2(2)}+\epsilon_{2(22)}$ \\
\hline 23 & & & & - & + & + & + & 2 & $e_{2(2)}+\epsilon_{3(22)}$ \\
\hline 24 & & & & + & + & - & + & 2 & $e_{2(2)}+\epsilon_{4(22)}$ \\
\hline 25 & - & - & + & - & - & + & + & 2 & $e_{3(2)}+\epsilon_{1(23)}$ \\
\hline 26 & & & & + & - & - & + & 2 & $e_{3(2)}+\epsilon_{2(23)}$ \\
\hline 27 & & & & - & + & - & + & 2 & $e_{3(2)}+\epsilon_{3(23)}$ \\
\hline 28 & & & & + & + & + & + & 2 & $e_{3(2)}+\epsilon_{4(23)}$ \\
\hline
\end{tabular}


Table A.2 (Cont'd)

\begin{tabular}{|c|c|c|c|c|c|c|c|c|c|}
\hline Run & A & B & $\mathrm{C}$ & $\mathrm{p}$ & $q$ & $\mathrm{r}$ & $\beta_{1}$ & Block & Error Term \\
\hline 29 & + & + & + & - & - & + & + & 2 & $e_{4(2)}+\epsilon_{1(24)}$ \\
\hline 30 & & & & + & - & - & + & 2 & $e_{4(2)}+\epsilon_{2(24)}$ \\
\hline 31 & & & & - & + & - & + & 2 & $e_{4(2)}+\epsilon_{3(24)}$ \\
\hline 32 & & & & + & + & + & t & 2 & $e_{4(2)}+\epsilon_{4(24)}$ \\
\hline
\end{tabular}


Table A.3: Standard Run Order of the $2^{(3+3)-(0+1) \pm(0+1)}$ BFFSP Design.

\begin{tabular}{||cccccccccc||}
\hline Run & A & B & C & p & q & r & $\delta_{1}$ & Block & Error Term \\
\hline 1 & - & - & - & - & - & + & - & 1 & $e_{1(1)}+\epsilon_{1(11)}$ \\
2 & & & & + & - & - & - & 1 & $e_{1(1)}+\epsilon_{2(11)}$ \\
3 & + & - & - & - & + & - & - & 1 & $e_{2(1)}+\epsilon_{1(12)}$ \\
4 & & & & + & + & + & - & 1 & $e_{2(1)}+\epsilon_{2(12)}$ \\
5 & - & + & - & - & + & - & - & 1 & $e_{3(1)}+\epsilon_{1(13)}$ \\
6 & & & & + & + & + & - & 1 & $e_{3(1)}+\epsilon_{2(13)}$ \\
7 & + & + & - & - & - & + & - & 1 & $e_{4(1)}+\epsilon_{1(14)}$ \\
8 & & & & + & - & - & - & 1 & $e_{4(1)}+\epsilon_{2(14)}$ \\
9 & - & - & + & - & - & - & - & 1 & $e_{5(1)}+\epsilon_{1(15)}$ \\
10 & & & & + & - & + & - & 1 & $e_{5(1)}+\epsilon_{2(15)}$ \\
11 & + & - & + & - & + & + & - & 1 & $e_{6(1)}+\epsilon_{1(16)}$ \\
12 & & & & + & + & - & - & 1 & $e_{6(1)}+\epsilon_{2(16)}$ \\
13 & - & + & + & - & + & + & - & 1 & $e_{7(1)}+\epsilon_{1(17)}$ \\
14 & & & & + & + & - & - & 1 & $e_{7(1)}+\epsilon_{2(17)}$ \\
15 & + & + & + & - & - & - & - & 1 & $e_{8(1)}+\epsilon_{1(18)}$ \\
16 & & & & + & - & + & - & 1 & $e_{8(1)}+\epsilon_{2(18)}$ \\
17 & - & - & - & - & + & + & + & 2 & $e_{1(2)}+\epsilon_{1(21)}$ \\
18 & & & & + & + & - & + & 2 & $e_{1(2)}+\epsilon_{2(21)}$ \\
19 & + & - & - & - & - & + & 2 & $e_{2(2)}+\epsilon_{1(22)}$ \\
20 & & & & + & - & + & + & 2 & $e_{2(2)}+\epsilon_{2(22)}$ \\
21 & - & + & - & - & - & - & + & 2 & $e_{3(2)}+\epsilon_{1(23)}$ \\
22 & & & & + & - & + & + & 2 & $e_{3(2)}+\epsilon_{2(23)}$ \\
23 & + & + & - & - & + & + & + & 2 & $e_{4(2)}+\epsilon_{1(24)}$ \\
24 & & & & + & + & - & + & 2 & $e_{4(2)}+\epsilon_{2(24)}$ \\
25 & - & - & + & - & + & - & + & 2 & $e_{5(2)}+\epsilon_{1(25)}$ \\
26 & & & & + & + & + & + & 2 & $e_{5(2)}+\epsilon_{2(25)}$ \\
27 & + & - & + & - & - & + & + & 2 & $e_{6(2)}+\epsilon_{1(26)}$ \\
28 & & & & + & - & - & + & 2 & $e_{6(2)}+\epsilon_{2(26)}$ \\
\hline
\end{tabular}


Table A.3 (Cont'd)

\begin{tabular}{||cccccccccc||}
\hline Run & A & B & C & p & q & r & $\beta_{1}$ & Block & Error Term \\
\hline 29 & - & + & + & - & - & + & + & 2 & $e_{7(2)}+\epsilon_{1(27)}$ \\
30 & & & & + & - & - & + & 2 & $e_{7(2)}+\epsilon_{2(27)}$ \\
31 & + & + & + & - & + & - & + & 2 & $e_{8(2)}+\epsilon_{1(28)}$ \\
32 & & & & + & + & + & + & 2 & $e_{8(2)}+\epsilon_{2(28)}$ \\
\hline
\end{tabular}


Table A.4: Standard Run Order of the $2^{(3+3)-(0+1) \pm(1+1)}$ BFFSP Design.

\begin{tabular}{||ccccccccccc||}
\hline Run & A & B & C & p & q & r & $\beta_{1}$ & $\delta_{1}$ & Block & Error Term \\
\hline 1 & - & - & - & + & - & - & - & - & 1 & $e_{1(1)}+\epsilon_{1(11)}$ \\
2 & & & & - & + & + & - & - & 1 & $e_{1(1)}+\epsilon_{2(11)}$ \\
3 & + & + & - & - & - & - & - & - & 1 & $e_{2(1)}+\epsilon_{1(12)}$ \\
4 & & & & + & + & + & - & - & 1 & $e_{2(1)}+\epsilon_{2(12)}$ \\
5 & + & - & + & - & - & + & - & - & 1 & $e_{3(1)}+\epsilon_{1(13)}$ \\
6 & & & & + & + & - & - & - & 1 & $e_{3(1)}+\epsilon_{2(13)}$ \\
7 & - & + & + & + & - & + & - & - & 1 & $e_{4(1)}+\epsilon_{1(14)}$ \\
8 & & & & - & + & - & - & - & 1 & $e_{4(1)}+\epsilon_{2(14)}$ \\
9 & + & - & - & + & - & + & + & - & 2 & $e_{1(2)}+\epsilon_{1(21)}$ \\
10 & & & & - & + & - & + & - & 2 & $e_{1(2)}+\epsilon_{2(21)}$ \\
11 & - & + & - & - & - & + & + & - & 2 & $e_{2(2)}+\epsilon_{1(22)}$ \\
12 & & & & + & + & - & + & - & 2 & $e_{2(2)}+\epsilon_{2(22)}$ \\
13 & - & - & + & - & - & - & + & - & 2 & $e_{3(2)}+\epsilon_{1(23)}$ \\
14 & & & & + & + & + & + & - & 2 & $e_{3(2)}+\epsilon_{2(23)}$ \\
15 & + & + & + & + & - & - & + & - & 2 & $e_{4(2)}+\epsilon_{1(24)}$ \\
16 & & & & - & + & + & + & - & 2 & $e_{4(2)}+\epsilon_{2(24)}$ \\
17 & - & - & - & - & - & - & - & + & 3 & $e_{1(3)}+\epsilon_{1(31)}$ \\
18 & & & & + & + & + & - & + & 3 & $e_{1(3)}+\epsilon_{2(31)}$ \\
19 & + & + & - & + & - & - & - & + & 3 & $e_{2(3)}+\epsilon_{1(32)}$ \\
20 & & & - & + & + & - & + & 3 & $e_{2(3)}+\epsilon_{2(32)}$ \\
21 & + & - & + & + & - & + & - & + & 3 & $e_{3(3)}+\epsilon_{1(33)}$ \\
22 & & & & - & + & - & - & + & 3 & $e_{3(3)}+\epsilon_{2(33)}$ \\
23 & - & + & + & - & - & + & - & + & 3 & $e_{4(3)}+\epsilon_{1(34)}$ \\
24 & & & & + & + & - & - & + & 3 & $e_{4(3)}+\epsilon_{2(34)}$ \\
25 & + & - & - & - & - & + & + & + & 4 & $e_{1(4)}+\epsilon_{1(41)}$ \\
26 & & & & + & + & - & + & + & 4 & $e_{1(4)}+\epsilon_{2(41)}$ \\
27 & - & + & - & + & - & + & + & + & 4 & $e_{2(4)}+\epsilon_{1(42)}$ \\
28 & & & & - & + & - & + & + & 4 & $e_{2(4)}+\epsilon_{2(42)}$ \\
\hline
\end{tabular}


Table A.4 (Cont'd)

\begin{tabular}{||ccccccccccc||}
\hline Run & A & B & C & p & q & r & $\beta_{1}$ & $\delta_{1}$ & Block & Error Term \\
\hline 29 & - & - & + & + & - & - & + & + & 4 & $e_{3(4)}+\epsilon_{1(43)}$ \\
30 & & & & - & + & + & + & + & 4 & $e_{3(4)}+\epsilon_{2(43)}$ \\
31 & + & + & + & - & - & - & + & + & 4 & $e_{4(4)}+\epsilon_{1(44)}$ \\
32 & & & & + & + & + & + & + & 4 & $e_{4(4)}+\epsilon_{2(44)}$ \\
\hline
\end{tabular}


Table A.5: Standard Run Order of the Impractical $2^{(4+2)-(0+1) \pm(2+1)}$ BFFSP Design.

\begin{tabular}{||cccccccccccc||}
\hline Run & $\mathrm{A}$ & $\mathrm{B}$ & $\mathrm{C}$ & $\mathrm{D}$ & $\mathrm{p}$ & $\mathrm{q}$ & $\beta_{1}$ & $\beta_{2}$ & $\delta_{1}$ & Block & Error Term \\
\hline 1 & - & - & - & - & - & - & - & - & - & 1 & $e_{1(1)}+\epsilon_{1(11)}$ \\
2 & + & - & + & - & + & + & - & - & - & 1 & $e_{2(1)}+\epsilon_{1(12)}$ \\
3 & + & + & - & + & - & + & - & - & - & 1 & $e_{3(1)}+\epsilon_{1(13)}$ \\
4 & - & + & + & + & + & - & - & - & - & 1 & $e_{4(1)}+\epsilon_{1(14)}$ \\
5 & - & + & - & - & + & - & + & - & - & 2 & $e_{1(2)}+\epsilon_{1(21)}$ \\
6 & + & + & + & - & - & + & + & - & - & 2 & $e_{2(2)}+\epsilon_{1(22)}$ \\
7 & + & - & - & + & + & + & + & - & - & 2 & $e_{3(2)}+\epsilon_{1(23)}$ \\
8 & - & - & + & + & - & - & + & - & - & 2 & $e_{4(2)}+\epsilon_{1(24)}$ \\
9 & + & + & - & - & - & - & - & + & - & 3 & $e_{1(3)}+\epsilon_{1(31)}$ \\
10 & - & + & + & - & + & + & - & + & - & 3 & $e_{2(3)}+\epsilon_{1(32)}$ \\
11 & - & - & - & + & - & + & - & + & - & 3 & $e_{3(3)}+\epsilon_{1(33)}$ \\
12 & + & - & + & + & + & - & - & + & - & 3 & $e_{4(3)}+\epsilon_{1(34)}$ \\
13 & + & - & - & - & + & - & + & + & - & 4 & $e_{1(4)}+\epsilon_{1(41)}$ \\
14 & - & - & + & - & - & + & + & + & - & 4 & $e_{2(4)}+\epsilon_{1(42)}$ \\
15 & - & + & - & + & + & + & + & + & - & 4 & $e_{3(4)}+\epsilon_{1(43)}$ \\
16 & + & + & + & + & - & - & + & + & - & 4 & $e_{4(4)}+\epsilon_{1(44)}$ \\
17 & - & - & - & - & + & + & - & - & + & 5 & $e_{1(5)}+\epsilon_{1(51)}$ \\
18 & + & - & + & - & - & - & - & - & + & 5 & $e_{2(5)}+\epsilon_{1(52)}$ \\
19 & + & + & - & + & + & - & - & - & + & 5 & $e_{3(5)}+\epsilon_{1(53)}$ \\
20 & - & + & + & + & - & + & - & - & + & 5 & $e_{4(5)}+\epsilon_{1(54)}$ \\
21 & - & + & - & - & - & + & + & - & + & 6 & $e_{1(6)}+\epsilon_{1(61)}$ \\
22 & + & + & + & - & + & - & + & - & + & 6 & $e_{2(6)}+\epsilon_{1(62)}$ \\
23 & + & - & - & + & - & + & + & - & + & 6 & $e_{3(6)}+\epsilon_{1(63)}$ \\
24 & - & - & + & + & + & + & + & - & + & 6 & $e_{4(6)}+\epsilon_{1(64)}$ \\
25 & + & + & - & - & + & + & - & + & + & 7 & $e_{1(7)}+\epsilon_{1(71)}$ \\
26 & - & + & + & - & - & - & - & + & + & 7 & $e_{2(7)}+\epsilon_{1(72)}$ \\
27 & - & - & - & + & + & - & - & + & + & 7 & $e_{3(7)}+\epsilon_{1(73)}$ \\
28 & + & - & + & + & - & + & - & + & + & 7 & $e_{4(7)}+\epsilon_{1(74)}$ \\
\hline
\end{tabular}


Table A.5 (Cont'd)

\begin{tabular}{||cccccccccccc||}
\hline Run & A & B & C & D & p & q & $\beta_{1}$ & $\beta_{2}$ & $\delta_{1}$ & Block & Error Term \\
\hline 29 & + & - & - & - & - & + & + & + & + & 8 & $e_{1(8)}+\epsilon_{1(81)}$ \\
30 & - & - & + & - & + & - & + & + & + & 8 & $e_{2(8)}+\epsilon_{1(82)}$ \\
31 & - & + & - & + & - & - & + & + & + & 8 & $e_{3(8)}+\epsilon_{1(83)}$ \\
32 & + & + & + & + & + & + & + & + & + & 8 & $e_{4(8)}+\epsilon_{1(84)}$ \\
\hline
\end{tabular}


Appendix B

Catalog of MA BFFSP Designs 


\section{B.1 Catalog of MA 8-Run BFFSP Designs via Pure WP Blocking}


Table B.1: MA 8-Run BFFSP Designs via Pure WP Blocking.

\begin{tabular}{|c|c|c|c|c|c|c|c|c|}
\hline$n$ & Design & WLP and Generators & (a) & (b) & (c) & (d) & (e) & (f) \\
\hline \multirow[t]{2}{*}{5} & 2,$2 ; 0,1 ; 1,0$ & 021 & 4 & 0 & 2 & 0 & 0 & 0 \\
\hline & & $A B \beta_{1}, A B p q$ & & & & & & \\
\hline \multirow[t]{2}{*}{6} & 2,$3 ; 0,2 ; 1,0$ & 2212 & 0 & 0 & 0 & 0 & 0 & 0 \\
\hline & & $A B \beta_{1}, A B p q, B p r$ & & & & & & \\
\hline \multirow[t]{2}{*}{7} & 2,$4 ; 0,3 ; 1,0$ & 43340000001 & 0 & 0 & 0 & 0 & 0 & 0 \\
\hline & & $A B \beta_{1}, A B p q, B p r, A p s$ & & & & & & \\
\hline
\end{tabular}

NOTE: The $2^{\left(n_{1}+n_{2}\right)-\left(k_{1}+k_{2}\right) \pm\left(b_{1}+b_{2}\right)}$ designs are labeled as "Design $=n_{1}, n_{2} ; k_{1}, k_{2} ; b_{1}, b_{2}$ " and are ordered by the number of treatment and blocking factors, $n=n_{1}+n_{2}+b_{1}+b_{2}$.

To save space the WLPs are truncated at the last non-zero value. A-G and $\mathrm{p}-\mathrm{v}$ are used to denote the WP and SP factors, respectively. The $i^{\text {th }} \mathrm{WP}$ blocking variable is denoted by $\beta_{i}$. Finally, the last letter in each generator represents the added factor.
(a) The number of clear main effects.
(b) The number of clear two-factor interactions.
(c) The number of clear SP main effects.
(d) The number of clear SP two-factor interactions.
(e) The number of clear SP main effects tested against WP error.
(f) The number of clear SP two-factor interactions tested against WP error. 


\section{B.2 Catalog of MA 16-Run BFFSP Designs via Pure WP Blocking}


Table B.2: MA 16-Run BFFSP Designs via Pure WP Blocking.

\begin{tabular}{|c|c|c|c|c|c|c|c|c|}
\hline$n$ & Design & WLP and Generators & (a) & (b) & (c) & (d) & (e) & (f) \\
\hline \multirow[t]{6}{*}{6} & 2,$3 ; 0,1 ; 1,0$ & 01011 & 5 & 9 & 3 & 9 & 0 & 0 \\
\hline & & $A B \beta_{1}, A B p q r$ & & & & & & \\
\hline & 3,$2 ; 0,1 ; 1,0$ & 0012 & 5 & 4 & 2 & 2 & 0 & 0 \\
\hline & & $A B C \beta_{1}, A B p q$ & & & & & & \\
\hline & 4,$1 ; 1,0 ; 1,0$ & 021 & 5 & 4 & 1 & 4 & 0 & 0 \\
\hline & & $A B C D, A B \beta_{1}$ & & & & & & \\
\hline \multirow[t]{10}{*}{7} & 2,$4 ; 0,2 ; 1,0$ & 023002 & 6 & 0 & 4 & 0 & 0 & 0 \\
\hline & & $A B \beta_{1}, B p q r, A p q s$ & & & & & & \\
\hline & 3,$2 ; 0,1 ; 2,0$ & 03031 & 5 & 7 & 2 & 7 & 0 & 1 \\
\hline & & $A B \beta_{1}, A C \beta_{2}, A B C p q$ & & & & & & \\
\hline & 3,$3 ; 0,2 ; 1,0$ & 0034 & 6 & 0 & 3 & 0 & 0 & 0 \\
\hline & & $A B C \beta_{1}, A B p q, A C p r$ & & & & & & \\
\hline & 4,$1 ; 1,0 ; 2,0$ & 061 & 5 & 4 & 1 & 4 & 0 & 0 \\
\hline & & $A B C D, A B \beta_{1}, A C \beta_{2}$ & & & & & & \\
\hline & 4,$2 ; 1,1 ; 1,0$ & 023002 & 6 & 0 & 2 & 0 & 0 & 0 \\
\hline & & $A B C D, A B \beta_{1}, A C p q$ & & & & & & \\
\hline
\end{tabular}

NOTE: The $2^{\left(n_{1}+n_{2}\right)-\left(k_{1}+k_{2}\right) \pm\left(b_{1}+b_{2}\right)}$ designs are labeled as "Design $=n_{1}, n_{2} ; k_{1}, k_{2} ; b_{1}, b_{2}$ " and are ordered by the number of treatment and blocking factors, $n=n_{1}+n_{2}+b_{1}+b_{2}$.

To save space the WLPs are truncated at the last non-zero value. A-G and p-v are used to denote the WP and SP factors, respectively. The $i^{\text {th }}$ WP blocking variable is denoted by $\beta_{i}$. Finally, the last letter in each generator represents the added factor.

(a) The number of clear main effects.

(b) The number of clear two-factor interactions.

(c) The number of clear SP main effects.

(d) The number of clear SP two-factor interactions.

(e) The number of clear SP main effects tested against WP error.

(f) The number of clear SP two-factor interactions tested against WP error. 
Table B.2 (Cont'd)

\begin{tabular}{|c|c|c|c|c|c|c|c|c|}
\hline$n$ & Design & WLP and Generators & (a) & (b) & (c) & (d) & (e) & (f) \\
\hline \multirow[t]{12}{*}{8} & 2,$5 ; 0,3 ; 1,0$ & 037004 & 7 & 0 & 5 & 0 & 0 & 0 \\
\hline & & $\begin{array}{l}A B \beta_{1}, B p q r, A p q s \\
A B q t\end{array}$ & & & & & & \\
\hline & 3,$3 ; 0,2 ; 2,0$ & 063006 & 6 & 0 & 3 & 0 & 0 & 0 \\
\hline & & $\begin{array}{l}A B \beta_{1}, A C \beta_{2}, A B p q \\
A C p r\end{array}$ & & & & & & \\
\hline & 3,$4 ; 0,3 ; 1,0$ & 007700000001 & 7 & 0 & 4 & 0 & 0 & 0 \\
\hline & & $\begin{array}{l}A B C \beta_{1}, A B p q, A C p r \\
B C p s\end{array}$ & & & & & & \\
\hline & 4,$2 ; 1,1 ; 2,0$ & 0730040001 & 6 & 0 & 2 & 0 & 0 & 0 \\
\hline & & $\begin{array}{l}A B C D, A B \beta_{1}, A C \beta_{2} \\
A B p q\end{array}$ & & & & & & \\
\hline & 4,$3 ; 1,2 ; 1,0$ & 0370040001 & 7 & 0 & 3 & 0 & 0 & 0 \\
\hline & & $\begin{array}{l}A B C D, A B \beta_{1}, A B p q \\
A C p r\end{array}$ & & & & & & \\
\hline & 5,$2 ; 2,1 ; 1,0$ & 223322000001 & 2 & 2 & 2 & 2 & 0 & 0 \\
\hline & & $\begin{array}{l}A B C D, A B E, A C \beta_{1} \\
B C p q\end{array}$ & & & & & & \\
\hline \multirow[t]{7}{*}{9} & 2,$6 ; 0,4 ; 1,0$ & 041400800041 & 8 & 0 & 6 & 0 & 0 & 0 \\
\hline & & $A B \beta_{1}, B p q r, A p q s$ & & & & & & \\
\hline & & $A B q t, A B p u$ & & & & & & \\
\hline & 3,$4 ; 0,3 ; 2,0$ & 09700120003 & 7 & 0 & 4 & 0 & 0 & 0 \\
\hline & & $\begin{array}{l}A B \beta_{1}, A C \beta_{2}, A B p q \\
A C p r, B C p s\end{array}$ & & & & & & \\
\hline & 3,$5 ; 0,4 ; 1,0$ & 317744001301 & 1 & 0 & 1 & 0 & 0 & 0 \\
\hline & & $\begin{array}{l}A B C \beta_{1}, A B C p q, A B p r \\
A C p s, B C p t\end{array}$ & & & & & & \\
\hline
\end{tabular}


Table B.2 (Cont'd)

\begin{tabular}{|c|c|c|c|c|c|c|c|c|}
\hline$n$ & Design & WLP and Generators & (a) & (b) & (c) & (d) & (e) & (f) \\
\hline \multirow[t]{4}{*}{9} & 4,$3 ; 1,2 ; 2,0$ & $\begin{array}{l}09700120003 \\
A B C D, A B \beta_{1}, A C \beta_{2} \\
A B p q, A C p r\end{array}$ & 7 & 0 & 3 & 0 & 0 & 0 \\
\hline & 4,$4 ; 1,3 ; 1,0$ & $\begin{array}{l}041400800041 \\
A B C D, A B \beta_{1}, A B p q \\
A C p r, B C p s\end{array}$ & 8 & 0 & 4 & 0 & 0 & 0 \\
\hline & 5,$3 ; 2,2 ; 1,0$ & $\begin{array}{l}337344041101 \\
A B C D, A B E, A C \beta_{1} \\
A B p q, A C p r\end{array}$ & 1 & 1 & 1 & 1 & 0 & 0 \\
\hline & 6,$2 ; 3,1 ; 1,0$ & $\begin{array}{l}44644004041 \\
A B C D, A B E, A C F \\
B C \beta_{1}, B C p q\end{array}$ & 2 & 0 & 2 & 0 & 0 & 0 \\
\hline \multirow[t]{5}{*}{10} & 2,$7 ; 0,5 ; 1,0$ & $\begin{array}{l}4414488084414 \\
A B \beta_{1}, p q r, A B p q s \\
A B q t, B p u, A p v\end{array}$ & 0 & 0 & 0 & 0 & 0 & 0 \\
\hline & 3,$5 ; 0,4 ; 2,0$ & $\begin{array}{l}39794120121303 \\
A B \beta_{1}, A C \beta_{2}, A B C p q \\
A B p r, A C p s, B C p t\end{array}$ & 1 & 1 & 1 & 1 & 0 & 1 \\
\hline & 3,$6 ; 0,5 ; 1,0$ & $\begin{array}{l}6210888442614 \\
A B C \beta_{1}, A B C p q, A B p r \\
A C p s, B C p t, C p u\end{array}$ & 0 & 0 & 0 & 0 & 0 & 0 \\
\hline & 4,$4 ; 1,3 ; 2,0$ & $\begin{array}{l}012140024000121 \\
A B C D, A B \beta_{1}, A C \beta_{2} \\
A B p q, A C p r, B C p s\end{array}$ & 8 & 0 & 4 & 0 & 0 & 0 \\
\hline & 4,$5 ; 1,4 ; 1,0$ & $\begin{array}{l}4414488084414 \\
A B C D, A B \beta_{1}, A B C p q \\
A B p r, A C p s, B C p t\end{array}$ & 0 & 0 & 0 & 0 & 0 & 0 \\
\hline
\end{tabular}


Table B.2 (Cont'd)

\begin{tabular}{lllcccccc}
\hline \hline$n$ & Design & WLP and Generators & (a) & (b) & (c) & (d) & (e) & (f) \\
\hline 10 & 5,$4 ; 2,3 ; 1,0$ & 4414488084414 & 0 & 0 & 0 & 0 & 0 & 0 \\
& & $A B C D, A B E, A C \beta_{1}$ & & & & & & \\
& & $A B p q, A C p r, B C p s$ & & & & & & \\
& 6,$3 ; 3,2 ; 1,0$ & 6410684482812 & 0 & 0 & 0 & 0 & 0 & 0 \\
& & $A B C D, A B E, A C F$ & & & & & & \\
& & $B C \beta 1, A B C p q, B C p r$ & & & & & & \\
\hline
\end{tabular}




\section{B.3 Catalog of MA 32-Run BFFSP Designs via Pure WP Blocking}


Table B.3: MA 32-Run BFFSP Designs via Pure WP Blocking.

\begin{tabular}{|c|c|c|c|c|c|c|c|c|}
\hline$n$ & Design & WLP and Generators & (a) & (b) & (c) & (d) & (e) & (f) \\
\hline \multirow[t]{8}{*}{7} & 2,$4 ; 0,1 ; 1,0$ & 0100011 & 6 & 14 & 4 & 14 & 0 & 0 \\
\hline & & $A B \beta_{1}, A B p q r s$ & & & & & & \\
\hline & 3,$3 ; 0,1 ; 1,0$ & 000111 & 6 & 15 & 3 & 12 & 0 & 0 \\
\hline & & $A B C \beta_{1}, A B p q r$ & & & & & & \\
\hline & 4,$2 ; 0,1 ; 1,0$ & 000111 & 6 & 15 & 2 & 9 & 0 & 1 \\
\hline & & $A B C D \beta_{1}, A B C p q$ & & & & & & \\
\hline & 4,$2 ; 1,0 ; 1,0$ & 021 & 6 & 9 & 2 & 9 & 0 & 0 \\
\hline & & $A B C D, A B \beta_{1}$ & & & & & & \\
\hline \multirow[t]{8}{*}{8} & 2,$5 ; 0,2 ; 1,0$ & 01112101 & 7 & 14 & 5 & 14 & 0 & 0 \\
\hline & & $A B \beta_{1}$, Bpqrs, ABpqt & & & & & & \\
\hline & 3,$3 ; 0,1 ; 2,0$ & 0300031 & 6 & 12 & 3 & 12 & 0 & 0 \\
\hline & & $A B \beta_{1}, A C \beta_{2}, A B C p q r$ & & & & & & \\
\hline & 3,$4 ; 0,2 ; 1,0$ & 001222 & 7 & 15 & 4 & 13 & 0 & 0 \\
\hline & & $A B C \beta_{1}, A B p q r, A C p q s$ & & & & & & \\
\hline & 4,$2 ; 0,1 ; 2,0$ & 010312 & 6 & 14 & 2 & 9 & 0 & 1 \\
\hline & & $A B C \beta_{1}, A B D \beta_{2}, A C D p q$ & & & & & & \\
\hline
\end{tabular}

NOTE: The $2^{\left(n_{1}+n_{2}\right)-\left(k_{1}+k_{2}\right) \pm\left(b_{1}+b_{2}\right)}$ designs are labeled as "Design $=n_{1}, n_{2} ; k_{1}, k_{2} ; b_{1}, b_{2}$ " and are ordered by the number of treatment and blocking factors, $n=n_{1}+n_{2}+b_{1}+b_{2}$.

To save space the WLPs are truncated at the last non-zero value. A-G and p-v are used to denote the WP and SP factors, respectively. The $i^{\text {th }}$ WP blocking variable is denoted by $\beta_{i}$. Finally, the last letter in each generator represents the added factor.
(a) The number of clear main effects.
(b) The number of clear two-factor interactions.
(c) The number of clear SP main effects.
(d) The number of clear SP two-factor interactions.
(e) The number of clear SP main effects tested against WP error.
(f) The number of clear SP two-factor interactions tested against WP error. 
Table B.3 (Cont'd)

\begin{tabular}{|c|c|c|c|c|c|c|c|c|}
\hline$n$ & Design & WLP and Generators & (a) & (b) & (c) & (d) & (e) & (f) \\
\hline \multirow[t]{12}{*}{8} & 4,$2 ; 1,0 ; 2,0$ & 061 & 6 & 9 & 2 & 9 & 0 & 0 \\
\hline & & $A B C D, A B \beta_{1}, A C \beta_{2}$ & & & & & & \\
\hline & 4,$3 ; 0,2 ; 1,0$ & 001222 & 7 & 15 & 3 & 10 & 0 & 2 \\
\hline & & $A B C D \beta_{1}, A B C p q, A B D p r$ & & & & & & \\
\hline & 4,$3 ; 1,1 ; 1,0$ & 02102002 & 7 & 15 & 3 & 15 & 0 & 0 \\
\hline & & $A B C D, A B \beta_{1}, A C p q r$ & & & & & & \\
\hline & 5,$2 ; 2,0 ; 1,0$ & 2212 & 2 & 11 & 2 & 11 & 0 & 0 \\
\hline & & $B C D, A C E, A B \beta_{1}$ & & & & & & \\
\hline & 5,$2 ; 1,1 ; 1,0$ & 001222 & 7 & 15 & 2 & 11 & 0 & 1 \\
\hline & & $A B D E, A C D \beta_{1}, B C D p q$ & & & & & & \\
\hline & 6,$1 ; 2,0 ; 1,0$ & 0034 & 7 & 6 & 1 & 6 & 0 & 0 \\
\hline & & $A B C E, A B D F, A C D \beta_{1}$ & & & & & & \\
\hline \multirow[t]{15}{*}{9} & 2,$6 ; 0,3 ; 1,0$ & 0132420201 & 8 & 12 & 6 & 12 & 0 & 0 \\
\hline & & $A B \beta_{1}, p q r s, A B p q t$ & & & & & & \\
\hline & & Bpru & & & & & & \\
\hline & 3,$4 ; 0,2 ; 2,0$ & 0314220201 & 7 & 12 & 4 & 12 & 0 & 1 \\
\hline & & $A B \beta_{1}, A C \beta_{2}, A B p q r$ & & & & & & \\
\hline & & Cpqs & & & & & & \\
\hline & 3,$5 ; 0,3 ; 1,0$ & 0033440000001 & 8 & 13 & 5 & 11 & 0 & 0 \\
\hline & & $A B C \beta_{1}, A B p q r, A C p q s$ & & & & & & \\
\hline & & $B C q t$ & & & & & & \\
\hline & 4,$2 ; 0,1 ; 3,0$ & 06041103 & 6 & 9 & 2 & 9 & 0 & 1 \\
\hline & & $A B \beta_{1}, A C \beta_{2}, A D \beta_{3}$ & & & & & & \\
\hline & & $A B C p q$ & & & & & & \\
\hline & 4,$3 ; 0,2 ; 2,0$ & 0116240001 & 7 & 14 & 3 & 10 & 0 & 2 \\
\hline & & $A B C \beta_{1}, A B D \beta_{2}, A C D p q$ & & & & & & \\
\hline & & $B C D p r$ & & & & & & \\
\hline
\end{tabular}


Table B.3 (Cont'd)

\begin{tabular}{|c|c|c|c|c|c|c|c|c|}
\hline$n$ & Design & WLP and Generators & (a) & (b) & (c) & (d) & (e) & (f) \\
\hline \multirow[t]{24}{*}{9} & 4,$4 ; 0,3 ; 1,0$ & 0033440000001 & 8 & 13 & 4 & 8 & 0 & 0 \\
\hline & & $A B D \beta_{1}, A B C p q, A C D p r$ & & & & & & \\
\hline & & $B D p s$ & & & & & & \\
\hline & * & $*$ & 8 & 13 & 4 & 10 & 0 & 3 \\
\hline & & $A B C D \beta_{1}, A B C p q, A B D p r$ & & & & & & \\
\hline & & $A C D p s$ & & & & & & \\
\hline & 4,$3 ; 1,1 ; 2,0$ & 061120040001 & 7 & 15 & 3 & 15 & 0 & 0 \\
\hline & & $A B C D, A B \beta_{1}, A C \beta_{2}$ & & & & & & \\
\hline & & $A B p q r$ & & & & & & \\
\hline & 4,$4 ; 1,2 ; 1,0$ & 023142020001 & 8 & 13 & 4 & 13 & 0 & 0 \\
\hline & & $A B C D, A B \beta_{1}, A B p q r$ & & & & & & \\
\hline & & $A C p q s$ & & & & & & \\
\hline & 5,$2 ; 1,1 ; 2,0$ & 021524000001 & 7 & 15 & 2 & 11 & 0 & 1 \\
\hline & & $A B C E, A B D \beta_{1}, A C D \beta_{2}$ & & & & & & \\
\hline & & $B C D p q$ & & & & & & \\
\hline & 5,$3 ; 1,2 ; 1,0$ & 003344000001 & 8 & 13 & 3 & 9 & 0 & 2 \\
\hline & & $A B C E, A B D \beta_{1}, A C D p q$ & & & & & & \\
\hline & & $B C D p r$ & & & & & & \\
\hline & 5,$3 ; 2,1 ; 1,0$ & 221221220000001 & 3 & 18 & 3 & 18 & 0 & 0 \\
\hline & & $A B C D, A B E, A C \beta_{1}$ & & & & & & \\
\hline & & $B C p q r$ & & & & & & \\
\hline & 6,$1 ; 2,0 ; 2,0$ & 0338000001 & 7 & 6 & 1 & 6 & 0 & 0 \\
\hline & & $A B C E, A B D F, A C D \beta_{1}$ & & & & & & \\
\hline & & $B C D \beta_{2}$ & & & & & & \\
\hline
\end{tabular}

* Indicates a design with $n_{1}, n_{2} ; k_{1}, k_{2} ; b_{1}, b_{2}$ and WLP identical to the design immediately preceding it in the table; the distinguishing design characteristic lies in (at least) one of the columns labeled (a) - (f). 
Table B.3 (Cont'd)

\begin{tabular}{|c|c|c|c|c|c|c|c|c|}
\hline$n$ & Design & WLP and Generators & (a) & (b) & (c) & (d) & (e) & (f) \\
\hline 9 & 6,$2 ; 2,1 ; 1,0$ & $\begin{array}{l}00344300000001 \\
A B C E, A B D F, A C D \beta_{1} \\
B C D p q\end{array}$ & 8 & 13 & 2 & 13 & 0 & 1 \\
\hline 10 & 2,$7 ; 0,4 ; 1,0$ & $\begin{array}{l}01648304031001 \\
A B \beta_{1}, \text { pqrs, } A B p q t \\
A B p r u, B q r v\end{array}$ & 9 & 7 & 7 & 7 & 0 & 0 \\
\hline & 3,$5 ; 0,3 ; 2,0$ & $\begin{array}{l}0338440405 \\
A B \beta_{1}, A C \beta_{2}, A B p q r \\
C p q s, A B C q t\end{array}$ & 8 & 10 & 5 & 10 & 0 & 0 \\
\hline & 3,$6 ; 0,4 ; 1,0$ & $\begin{array}{l}006488000014 \\
A B C \beta_{1}, A B p q r, A C p q s \\
B C q t, B C p u\end{array}$ & 9 & 8 & 6 & 6 & 0 & 0 \\
\hline & 4,$3 ; 0,2 ; 3,0$ & $\begin{array}{l}0718260601 \\
C D \beta_{1}, B D \beta_{2}, A D \beta_{3} \\
A B D p q, A C p r\end{array}$ & 7 & 10 & 3 & 10 & 0 & 2 \\
\hline & 4,$4 ; 0,3 ; 2,0$ & $\begin{array}{l}0131048000302 \\
A B C \beta_{1}, A B D \beta_{2}, A C D p q \\
B C D p r, A B p s\end{array}$ & 8 & 12 & 4 & 8 & 0 & 0 \\
\hline & 4,$4 ; 1,2 ; 2,0$ & $\begin{array}{l}073244080102 \\
A B C D, A B \beta_{1}, A C \beta_{2} \\
A B p q r, A C p q s\end{array}$ & 8 & 13 & 4 & 13 & 0 & 0 \\
\hline & 4,$5 ; 0,4 ; 1,0$ & $\begin{array}{l}006488000014 \\
A B C D \beta_{1}, A B C p q, A B D p r \\
A C D p s, B C D p t\end{array}$ & 9 & 8 & 5 & 8 & 0 & 4 \\
\hline & 4,$5 ; 1,3 ; 1,0$ & $\begin{array}{l}026284040212 \\
A B C D, A B \beta_{1}, A B p q r \\
A C p q s, B C q t\end{array}$ & 9 & 8 & 5 & 8 & 0 & 0 \\
\hline
\end{tabular}


Table B.3 (Cont'd)

\begin{tabular}{|c|c|c|c|c|c|c|c|c|}
\hline$n$ & Design & WLP and Generators & (a) & (b) & (c) & (d) & (e) & (f) \\
\hline \multirow[t]{9}{*}{10} & 5,$2 ; 1,1 ; 3,0$ & $\begin{array}{l}0101524080001 \\
A B C E, A B C D \beta_{1}, A B \beta_{2} \\
A D \beta_{3}, A B D p q\end{array}$ & 7 & 11 & 2 & 11 & 0 & 1 \\
\hline & 5,$3 ; 1,2 ; 2,0$ & $\begin{array}{l}025904260201 \\
A B C E, A B D \beta_{1}, A C D \beta_{2} \\
A B C D p q, A B p r\end{array}$ & 8 & 4 & 3 & 2 & 0 & 0 \\
\hline & 5,$4 ; 1,3 ; 1,0$ & $\begin{array}{l}006488000014 \\
A B C E, A B D \beta_{1}, A C D p q \\
B C D p r, A B p s\end{array}$ & 9 & 8 & 4 & 4 & 0 & 0 \\
\hline & 5,$4 ; 2,2 ; 1,0$ & $\begin{array}{l}224462220412 \\
A B C D, A B E, A C \beta_{1} \\
A B p q r, A C p q s\end{array}$ & 4 & 11 & 4 & 11 & 0 & 0 \\
\hline & 6,$1 ; 2,0 ; 3,0$ & $\begin{array}{l}015300120001 \\
A B C E, A B D F, A B C D \beta_{1} \\
A B \beta_{2}, A C \beta_{3}\end{array}$ & 7 & 6 & 1 & 6 & 0 & 0 \\
\hline & 6,$2 ; 2,1 ; 2,0$ & $\begin{array}{l}0351002240302 \\
A B C E, A B D F, A C D \beta_{1} \\
B C D \beta_{2}, A B C D p q\end{array}$ & 8 & 4 & 2 & 4 & 0 & 0 \\
\hline & 6,$3 ; 2,2 ; 1,0$ & $\begin{array}{l}009700660003 \\
A B C E, A B D F, A C D \beta_{1} \\
A B C D p q, A C p r\end{array}$ & 9 & 0 & 3 & 0 & 0 & 0 \\
\hline & 6,$3 ; 3,1 ; 1,0$ & $\begin{array}{l}4335304005031 \\
A B C D, A B E, A C F \\
B C \beta_{1}, B C p q r\end{array}$ & 3 & 21 & 3 & 21 & 0 & 0 \\
\hline & 7,$2 ; 3,1 ; 1,0$ & $\begin{array}{l}0010800440014 \\
A B C E, A B D F, A C D G \\
B C D \beta_{1}, A B C D p q\end{array}$ & 9 & 2 & 2 & 2 & 0 & 0 \\
\hline
\end{tabular}




\section{B.4 Catalog of MA 8-Run BFFSP Designs via Separation}


Table B.4: MA 8-Run BFFSP Designs via Separation.

\begin{tabular}{|c|c|c|c|c|c|c|c|c|}
\hline$n$ & Design & WLP and Generators & (a) & (b) & (c) & (d) & (e) & (f) \\
\hline \multirow[t]{2}{*}{5} & 1,$3 ; 0,1 ; 0,1$ & 021 & 4 & 0 & 3 & 0 & 1 & 0 \\
\hline & & $A p q r, p q \delta_{1}$ & & & & & & \\
\hline \multirow[t]{2}{*}{6} & 1,$4 ; 0,2 ; 0,1$ & 2212 & 0 & 0 & 0 & 0 & 0 & 0 \\
\hline & & $p q r, A p q s, A q \delta_{1}$ & & & & & & \\
\hline \multirow[t]{2}{*}{7} & 1,$5 ; 0,3 ; 0,1$ & 433400000001 & 0 & 0 & 0 & 0 & 0 & 0 \\
\hline & & $p q r, A p q s, A q t, A p \delta_{1}$ & & & & & & \\
\hline
\end{tabular}

NOTE: The $2^{\left(n_{1}+n_{2}\right)-\left(k_{1}+k_{2}\right) \pm\left(b_{1}+b_{2}\right)}$ designs are labeled as "Design $=n_{1}, n_{2} ; k_{1}, k_{2} ; b_{1}, b_{2}$ " and are ordered by the number of treatment and blocking factors, $n=n_{1}+n_{2}+b_{1}+b_{2}$.

To save space the WLPs are truncated at the last non-zero value. A-G and p-v are used to denote the WP and SP factors, respectively. The $j^{\text {th }}$ separator is denoted by $\delta_{j}$. Finally, the last letter in each generator represents the added factor.

(a) The number of clear main effects.

(b) The number of clear two-factor interactions.

(c) The number of clear SP main effects.

(d) The number of clear SP two-factor interactions.

(e) The number of clear SP main effects tested against WP error.

(f) The number of clear SP two-factor interactions tested against WP error. 


\section{B.5 Catalog of MA 16-Run BFFSP Designs via Separation}


Table B.5: MA 16-Run BFFSP Designs via Separation.

\begin{tabular}{|c|c|c|c|c|c|c|c|c|}
\hline$n$ & Design & WLP and Generators & (a) & (b) & (c) & (d) & (e) & (f) \\
\hline 6 & 1,$4 ; 0,1 ; 0,1$ & 0012 & 5 & 4 & 4 & 4 & 0 & 0 \\
\hline & & $p q r s, A p q \delta_{1}$ & & & & & & \\
\hline & 2,$3 ; 0,1 ; 0,1$ & 0012 & 5 & 4 & 3 & 4 & 0 & 2 \\
\hline & & $A B q r, B p q \delta_{1}$ & & & & & & \\
\hline & * & * & 5 & 4 & 3 & 3 & 1 & 1 \\
\hline & & $B p q r, A p q \delta_{1}$ & & & & & & \\
\hline & 3,$2 ; 1,0 ; 0,1$ & 100101 & 2 & 7 & 2 & 7 & 0 & 1 \\
\hline & & $A B C, A B p q \delta_{1}$ & & & & & & \\
\hline \multirow[t]{8}{*}{7} & 1,$4 ; 0,1 ; 0,2$ & 0214 & 5 & 4 & 4 & 4 & 0 & 0 \\
\hline & & $p q r s, p q \delta_{1}, A p r \delta_{2}$ & & & & & & \\
\hline & 1,$5 ; 0,2 ; 0,1$ & 0034 & 6 & 0 & 5 & 0 & 0 & 0 \\
\hline & & $p q r s, A p q t, A p r \delta_{1}$ & & & & & & \\
\hline & 2,$4 ; 0,2 ; 0,1$ & 0034 & 6 & 0 & 4 & 0 & 0 & 0 \\
\hline & & $A B q r, A B p s, B p q \delta_{1}$ & & & & & & \\
\hline & 3,$3 ; 1,1 ; 0,1$ & 111211 & 3 & 5 & 3 & 5 & 1 & 1 \\
\hline & & $A B C, A B p q r, B p q \delta_{1}$ & & & & & & \\
\hline
\end{tabular}

NOTE: The $2^{\left(n_{1}+n_{2}\right)-\left(k_{1}+k_{2}\right) \pm\left(b_{1}+b_{2}\right)}$ designs are labeled as "Design $=n_{1}, n_{2} ; k_{1}, k_{2} ; b_{1}, b_{2}$ " and are ordered by the number of treatment and blocking factors, $n=n_{1}+n_{2}+b_{1}+b_{2}$.

To save space the WLPs are truncated at the last non-zero value. A-G and $p-w$ are used to denote the WP and SP factors, respectively. The $j^{\text {th }}$ separator is denoted by $\delta_{j}$. Finally, the last letter in each generator represents the added factor.

(a) The number of clear main effects.

(b) The number of clear two-factor interactions.

(c) The number of clear SP main effects.

(d) The number of clear SP two-factor interactions.

(e) The number of clear SP main effects tested against WP error.

(f) The number of clear SP two-factor interactions tested against WP error.

* Indicates a design with $n_{1}, n_{2} ; k_{1}, k_{2} ; b_{1}, b_{2}$ and WLP identical to the design immediately preceding it in the table; the distinguishing design characteristic lies in (at least) one of the columns labeled (a) - (f). 
Table B.5 (Cont'd)

\begin{tabular}{|c|c|c|c|c|c|c|c|c|}
\hline$n$ & Design & WLP and Generators & (a) & (b) & (c) & (d) & (e) & (f) \\
\hline \multirow[t]{5}{*}{8} & 1,$5 ; 0,2 ; 0,2$ & 0338000001 & 6 & 0 & 5 & 0 & 1 & 0 \\
\hline & & $\begin{array}{l}p q r s, A p q t, p q \delta_{1} \\
\text { Apr } \delta_{2}\end{array}$ & & & & & & \\
\hline & 1,$6 ; 0,3 ; 0,1$ & $\begin{array}{l}007700000001 \\
\text { pqrs, Apqt, Apru } \\
\text { Aqr } \delta_{1}\end{array}$ & 7 & 0 & 6 & 0 & 0 & 0 \\
\hline & 2,$5 ; 0,3 ; 0,1$ & $\begin{array}{l}007700000001 \\
B p q r, A p q s, A B q t \\
A B p \delta_{1}\end{array}$ & 7 & 0 & 5 & 0 & 1 & 0 \\
\hline & 3,$4 ; 1,2 ; 0,1$ & $\begin{array}{l}2134220001 \\
A B C, A B p q r, B p q s \\
A q \delta_{1}\end{array}$ & 2 & 1 & 2 & 1 & 1 & 0 \\
\hline \multirow[t]{4}{*}{9} & 1,$6 ; 0,3 ; 0,2$ & $\begin{array}{l}09700120003 \\
p q r s, A p q t, \text { Apru } \\
p q \delta_{1}, p r \delta_{2}\end{array}$ & 7 & 0 & 6 & 0 & 2 & 0 \\
\hline & 1,$7 ; 0,4 ; 0,1$ & 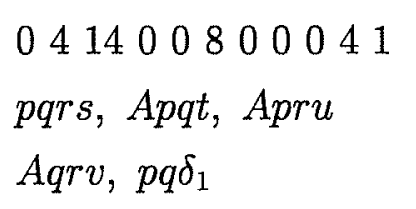 & 8 & 0 & 7 & 0 & 1 & 0 \\
\hline & 2,$6 ; 0,4 ; 0,1$ & $\begin{array}{l}041400800041 \\
B p q r, A p q s, A B q t \\
A B p u, p q \delta_{1}\end{array}$ & 8 & 0 & 6 & 0 & 2 & 0 \\
\hline & 3,$5 ; 1,3 ; 0,1$ & $\begin{array}{l}317744001301 \\
A B C, A B p q r, B p q s \\
A q t, A p \delta_{1}\end{array}$ & 1 & 0 & 1 & 0 & 1 & 0 \\
\hline
\end{tabular}


Table B.5 (Cont'd)

\begin{tabular}{|c|c|c|c|c|c|c|c|c|}
\hline$n$ & Design & WLP and Generators & (a) & (b) & (c) & (d) & (e) & (f) \\
\hline \multirow[t]{12}{*}{10} & 1,$7 ; 0,4 ; 0,2$ & 01214000240000121 & 8 & 0 & 7 & 0 & 3 & 0 \\
\hline & & $p q r s$, Apqt, Apru & & & & & & \\
\hline & & $A q r v, p q \delta_{1}, p r \delta_{2}$ & & & & & & \\
\hline & 1,$8 ; 0,5 ; 0,1$ & 4414488084414 & 0 & 0 & 0 & 0 & 0 & 0 \\
\hline & & $p q r s, p q t, A p q u$ & & & & & & \\
\hline & & $A p r v, A q r w, p r \delta_{1}$ & & & & & & \\
\hline & 2,$7 ; 0,5 ; 0,1$ & 4414488084414 & 0 & 0 & 0 & 0 & 0 & 0 \\
\hline & & $p q r, A B p q s, A B q t$ & & & & & & \\
\hline & & $B p u, A p v, B p q \delta_{1}$ & & & & & & \\
\hline & 3,$6 ; 1,4 ; 0,1$ & 4414488084414 & 0 & 0 & 0 & 0 & 0 & 0 \\
\hline & & $A B C, A B p q r, B p q s$ & & & & & & \\
\hline & & $A q t, A p u, p q \delta_{1}$ & & & & & & \\
\hline
\end{tabular}




\section{B.6 Catalog of MA 32-Run BFFSP Designs via Separation}


Table B.6: MA 32-Run BFFSP Designs via Separation.

\begin{tabular}{|c|c|c|c|c|c|c|c|c|}
\hline$n$ & Design & WLP and Generators & (a) & (b) & (c) & (d) & (e) & (f) \\
\hline \multirow[t]{10}{*}{7} & 1,$5 ; 0,1 ; 0,1$ & 000111 & 6 & 15 & 5 & 15 & 0 & 0 \\
\hline & & Apqrt, $p q s \delta_{1}$ & & & & & & \\
\hline & 2,$4 ; 0,1 ; 0,1$ & 000111 & 6 & 15 & 4 & 14 & 0 & 1 \\
\hline & & $A B p q s, p q r \delta_{1}$ & & & & & & \\
\hline & 3,$3 ; 0,1 ; 0,1$ & 000111 & 6 & 15 & 3 & 12 & 0 & 3 \\
\hline & & $A B C q r, A C p q \delta_{1}$ & & & & & & \\
\hline & 3,$3 ; 1,0 ; 0,1$ & 1000000101 & 3 & 12 & 3 & 12 & 0 & 0 \\
\hline & & $A B C, A B p q r \delta_{1}$ & & & & & & \\
\hline & 4,$2 ; 1,0 ; 0,1$ & 0010002 & 6 & 9 & 2 & 9 & 0 & 1 \\
\hline & & $A B C D, A B p q \delta_{1}$ & & & & & & \\
\hline \multirow[t]{4}{*}{8} & 1,$5 ; 0,1 ; 0,2$ & 001402 & 6 & 9 & 5 & 9 & 0 & 0 \\
\hline & & $p q r t, p q s \delta_{1}, A p r s \delta_{2}$ & & & & & & \\
\hline & 1,$6 ; 0,2 ; 0,1$ & 001222 & 7 & 15 & 6 & 15 & 0 & 0 \\
\hline & & pqrt, Aprsu, qrs $\delta_{1}$ & & & & & & \\
\hline
\end{tabular}

NOTE: The $2^{\left(n_{1}+n_{2}\right)-\left(k_{1}+k_{2}\right) \pm\left(b_{1}+b_{2}\right)}$ designs are labeled as "Design $=n_{1}, n_{2} ; k_{1}, k_{2} ; b_{1}, b_{2}$ " and are ordered by the number of treatment and blocking factors, $n=n_{1}+n_{2}+b_{1}+b_{2}$.

To save space the WLPs are truncated at the last non-zero value. A-G and $\mathrm{p}-\mathrm{w}$ are used to denote the WP and SP factors, respectively. The $j^{\text {th }}$ separator is denoted by $\delta_{j}$. Finally, the last letter in each generator represents the added factor.

(a) The number of clear main effects.

(b) The number of clear two-factor interactions.

(c) The number of clear SP main effects.

(d) The number of clear SP two-factor interactions.

(e) The number of clear SP main effects tested against WP error.

(f) The number of clear SP two-factor interactions tested against WP error.

* Indicates a design with $n_{1}, n_{2} ; k_{1}, k_{2} ; b_{1}, b_{2}$ and WLP identical to the design immediately preceding it in the table; the distinguishing design characteristic lies in (at least) one of the columns labeled (a) - (f). 
Table B.6 (Cont'd)

\begin{tabular}{|c|c|c|c|c|c|c|c|c|}
\hline$n$ & Design & WLP and Generators & (a) & (b) & (c) & (d) & (e) & (f) \\
\hline \multirow[t]{24}{*}{8} & 2,$4 ; 0,1 ; 0,2$ & 001402 & 6 & 9 & 4 & 8 & 0 & 0 \\
\hline & & $p q r s, A B p q \delta_{1}, B p r \delta_{2}$ & & & & & & \\
\hline & * & $*$ & 6 & 9 & 4 & 9 & 0 & 5 \\
\hline & & $A B r s, A p r \delta_{1}, A B p q \delta_{2}$ & & & & & & \\
\hline & * & $*$ & 6 & 9 & 4 & 9 & 1 & 4 \\
\hline & & $A B r s, B p q r \delta_{1}, A p r \delta_{2}$ & & & & & & \\
\hline & 2,$5 ; 0,2 ; 0,1$ & 001222 & 7 & 15 & 5 & 14 & 0 & 0 \\
\hline & & $p q r s, A B p q t, A B p r \delta_{1}$ & & & & & & \\
\hline & $*$ & $*$ & 7 & 15 & 5 & 15 & 0 & 2 \\
\hline & & $A B r s, A B p q t, A p q r \delta_{1}$ & & & & & & \\
\hline & 3,$4 ; 0,2 ; 0,1$ & 001222 & 7 & 15 & 4 & 12 & 0 & 0 \\
\hline & & $A B C p r, A B C q s, A B p q \delta_{1}$ & & & & & & \\
\hline & * & $*$ & 7 & 15 & 4 & 13 & 0 & 5 \\
\hline & & $A B C p r, A C q s, B C p q \delta_{1}$ & & & & & & \\
\hline & $*$ & * & 7 & 15 & 4 & 13 & 2 & 3 \\
\hline & & $A B p q r, A C p q s, B C p q \delta_{1}$ & & & & & & \\
\hline & 3,$3 ; 1,0 ; 0,2$ & 100303 & 3 & 12 & 3 & 12 & 0 & 3 \\
\hline & & $A B C, A B p q \delta_{1}, B p r \delta_{2}$ & & & & & & \\
\hline & 3,$4 ; 1,1 ; 0,1$ & 1002121 & 4 & 18 & 4 & 18 & 0 & 2 \\
\hline & & $A B C, A B p q r s, B p q \delta_{1}$ & & & & & & \\
\hline & 4,$3 ; 1,1 ; 0,1$ & 001222 & 7 & 15 & 3 & 15 & 1 & 5 \\
\hline & & $A B C D, A B p q r, A C p q \delta_{1}$ & & & & & & \\
\hline & 5,$2 ; 2,0 ; 0,1$ & 20100202 & 2 & 11 & 2 & 11 & 0 & 1 \\
\hline & & $A B C D, A B E, A C p q \delta_{1}$ & & & & & & \\
\hline 9 & 1,$5 ; 0,1 ; 0,3$ & 0308031 & 6 & 12 & 5 & 12 & 1 & 4 \\
\hline & & Apqrst, $A p q \delta_{1}, A r \delta_{2}$ & & & & & & \\
\hline & & $\mathrm{Aps}_{3}$ & & & & & & \\
\hline
\end{tabular}


Table B.6 (Cont'd)

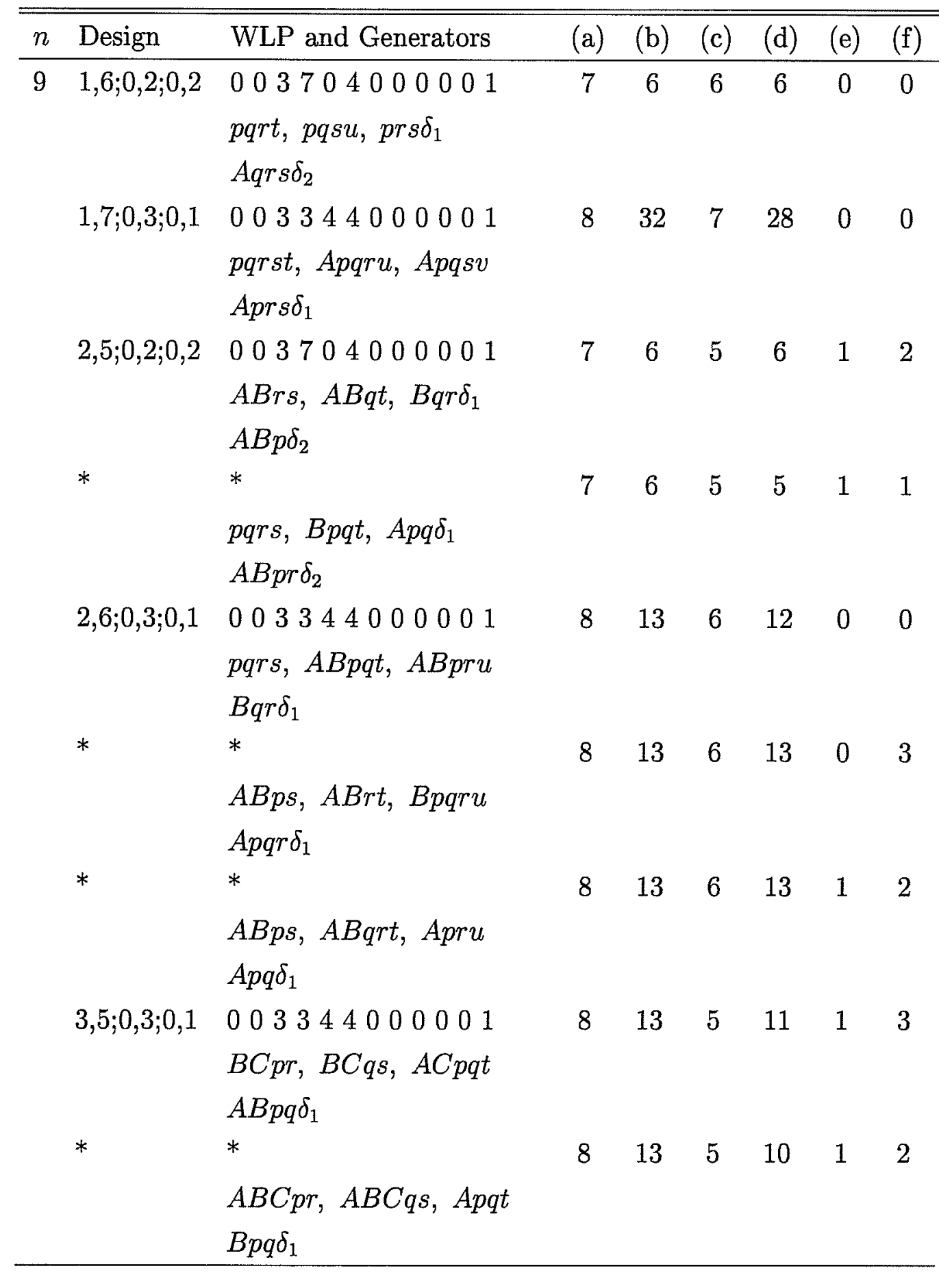


Table B.6 (Cont'd)

\begin{tabular}{|c|c|c|c|c|c|c|c|c|}
\hline$n$ & Design & WLP and Generators & (a) & (b) & (c) & (d) & (e) & (f) \\
\hline \multirow[t]{13}{*}{9} & $*$ & $*$ & 8 & 13 & 5 & 13 & 0 & 7 \\
\hline & & $\begin{array}{l}B C p r, A B C q s, A B p t \\
A C p q \delta_{1}\end{array}$ & & & & & & \\
\hline & $*$ & $*$ & 8 & 13 & 5 & 13 & 1 & 6 \\
\hline & & $\begin{array}{l}A B p q r, A C p q s, B C p q t \\
A B C q \delta_{1}\end{array}$ & & & & & & \\
\hline & 3,$4 ; 1,1 ; 0,2$ & 101606001 & 4 & 12 & 4 & 12 & 0 & 0 \\
\hline & & $\begin{array}{l}A B C, p q r s, A B p q \delta_{1} \\
B p r \delta_{2}\end{array}$ & & & & & & \\
\hline & 3,$5 ; 1,2 ; 0,1$ & 1023331101 & 5 & 13 & 5 & 13 & 0 & 2 \\
\hline & & $\begin{array}{l}A B C, A B p q r s, B p q t \\
B p r \delta_{1}\end{array}$ & & & & & & \\
\hline & 4,$4 ; 1,2 ; 0,1$ & 003344000001 & 8 & 13 & 4 & 13 & 1 & 6 \\
\hline & & $\begin{array}{l}A B C D, A B p q r, A C p q s \\
B C q \delta_{1}\end{array}$ & & & & & & \\
\hline & 5,$3 ; 2,1 ; 0,1$ & 201324200001 & 3 & 18 & 3 & 18 & 1 & 6 \\
\hline & & $A B C D, A B E, A C p q r$ & & & & & & \\
\hline & & $A B p q \delta_{1}$ & & & & & & \\
\hline \multirow[t]{5}{*}{10} & 1,$6 ; 0,2 ; 0,3$ & 05112260203 & 7 & 12 & 6 & 12 & 2 & 0 \\
\hline & & $\begin{array}{l}\text { Aqrst, Aprsu, Apqs } \delta_{1} \\
r s \delta_{2}, A r \delta_{3}\end{array}$ & & & & & & \\
\hline & * & $*$ & 7 & 12 & 6 & 12 & 1 & 6 \\
\hline & & Arst, Apqsu, Ar $\delta_{1}$ & & & & & & \\
\hline & & $A p s \delta_{2}, A p q r \delta_{3}$ & & & & & & \\
\hline
\end{tabular}


Table B.6 (Cont'd)

\begin{tabular}{|c|c|c|c|c|c|c|c|c|}
\hline$n$ & Design & WLP and Generators & (a) & (b) & (c) & (d) & (e) & (f) \\
\hline \multirow[t]{27}{*}{10} & 1,$7 ; 0,3 ; 0,2$ & 01310480000302 & 8 & 12 & 7 & 12 & 1 & 0 \\
\hline & & Aqrst, prsu, Apqsv & & & & & & \\
\hline & & $A q \delta_{1}, A p r \delta_{2}$ & & & & & & \\
\hline & $*$ & $*$ & 8 & 12 & 7 & 12 & 0 & 2 \\
\hline & & pqrst, Apqru, Apqsv & & & & & & \\
\hline & & $p q \delta_{1}, A p r s \delta_{2}$ & & & & & & \\
\hline & 1,$8 ; 0,4 ; 0,1$ & 006488000014 & 9 & 8 & 8 & 8 & 0 & 0 \\
\hline & & Aqrst, Aprsu, Apqrv & & & & & & \\
\hline & & Apqsw, $p q r s \delta_{1}$ & & & & & & \\
\hline & 2,$6 ; 0,3 ; 0,2$ & 0131048000302 & 8 & 12 & 6 & 12 & 0 & 0 \\
\hline & & pqrs, ABpqt, ABpru & & & & & & \\
\hline & & $B q r \delta_{1}, A q r \delta_{2}$ & & & & & & \\
\hline & 2,$7 ; 0,4 ; 0,1$ & 006488000014 & 9 & 8 & 7 & 8 & 0 & 2 \\
\hline & & $A B p s, A B q r t, A p r u$ & & & & & & \\
\hline & & $B p q r v, A p q \delta_{1}$ & & & & & & \\
\hline & * & $*$ & 9 & 8 & 7 & 7 & 1 & 1 \\
\hline & & pqrs, ABpqt, ABpru & & & & & & \\
\hline & & $B q r v, A q r \delta_{1}$ & & & & & & \\
\hline & 3,$6 ; 0,4 ; 0,1$ & 006488000014 & 9 & 8 & 6 & 8 & 1 & 4 \\
\hline & & $A B p q r, A C p q s, B C p q t$ & & & & & & \\
\hline & & $A B C q u, A B C p \delta_{1}$ & & & & & & \\
\hline & * & $*$ & 9 & 8 & 6 & 6 & 2 & 2 \\
\hline & & $B C p r, B p q s, A C p q t$ & & & & & & \\
\hline & & $A B C q u, A B p \delta_{1}$ & & & & & & \\
\hline & 3,$5 ; 1,2 ; 0,2$ & 113928021301 & 5 & 9 & 5 & 9 & 1 & 1 \\
\hline & & $A B C, p q r s, A B p q t$ & & & & & & \\
\hline & & $B p q \delta_{1}, A B p r \delta_{2}$ & & & & & & \\
\hline
\end{tabular}


Table B.6 (Cont'd)

\begin{tabular}{lllcccccc}
\hline \hline$n$ & Design & WLP and Generators & (a) & (b) & (c) & (d) & (e) & (f) \\
\hline 10 & 3,$6 ; 1,3 ; 0,1$ & 10556522120101 & 6 & 9 & 6 & 9 & 0 & 1 \\
& & $A B C, p q r s, A B p q t$ & & & & & & \\
& $B p r u, A B q r \delta_{1}$ & & & & & & \\
5,$5 ; 1,3 ; 0,1$ & 006488000014 & 9 & 8 & 5 & 8 & 1 & 4 \\
& $A B C D, A B p q r, A C p q s$ & & & & & & \\
& $B C q t, B C p \delta_{1}$ & & & & & & \\
5,$4 ; 2,2 ; 0,1$ & 204466220212 & 4 & 11 & 4 & 11 & 1 & 5 \\
& $A B C D, A B E, A B p q r$ & & & & & & \\
& $A C p q s, B C q \delta_{1}$ & & & & & & \\
6,$3 ; 3,1 ; 0,1$ & 40442622212 & 3 & 15 & 3 & 15 & 1 & 5 \\
& $A B C D, A B E, A C F$ & & & & & \\
& $A B C p q r, A p q \delta_{1}$ & & & & & \\
\hline
\end{tabular}




\section{B.7 Catalog of MA 16-Run BFFSP Designs via Mixed Blocking}


Table B.7: MA 16-Run BFFSP Designs via Mixed Blocking.

\begin{tabular}{lllcccccc}
\hline \hline$n$ & Design & WLP and Generators & (a) & (b) & (c) & (d) & (e) & (f) \\
\hline 7 & 2,$3 ; 0,1 ; 1,1$ & 0214 & 5 & 4 & 3 & 4 & 0 & 2 \\
& & $A B \beta_{1}, A B q r, B p q \delta_{1}$ & & & & & & \\
& & & & & & & \\
8 & 2,$4 ; 0,2 ; 1,1$ & 0338000001 & 6 & 0 & 4 & 0 & 0 & 0 \\
& & $A B \beta_{1}, A B q r, A B p s$ & & & & & & \\
& $B p q \delta_{1}$ & & & & & & \\
& & & & & & & \\
9 & 2,$5 ; 0,3 ; 1,1$ & 09700120003 & 7 & 0 & 5 & 0 & 1 & 0 \\
& & $A B \beta_{1}, A B p r, A B q s$ & & & & & & \\
& & $A p q t, A B p q \delta_{1}$ & & & & & & \\
10 & 2,$6 ; 0,4 ; 1,1$ & 012140024000121 & 8 & 0 & 6 & 0 & 2 & 0 \\
& & $A B \beta_{1}, B p q r, A p q s$ & & & & & & \\
& & $A B q t, A B p u, p q \delta_{1}$ & & & & & & \\
\hline
\end{tabular}

NOTE: The $2^{\left(n_{1}+n_{2}\right)-\left(k_{1}+k_{2}\right) \pm\left(b_{1}+b_{2}\right)}$ designs are labeled as "Design $=n_{1}, n_{2} ; k_{1}, k_{2} ; b_{1}, b_{2}$ " and are ordered by the number of treatment and blocking factors, $n=n_{1}+n_{2}+b_{1}+b_{2}$.

To save space the WLPs are truncated at the last non-zero value. A-G and $p-v$ are used to denote the WP and SP factors, respectively. The $i^{t h}$ and $j^{t h}$ WP blocking variable and separator are denoted by $\beta_{i}$ and $\delta_{j}$, respectively. Finally, the last letter in each generator represents the added factor.

(a) The number of clear main effects.

(b) The number of clear two-factor interactions.

(c) The number of clear SP main effects.

(d) The number of clear SP two-factor interactions.

(e) The number of clear SP main effects tested against WP error.

(f) The number of clear SP two-factor interactions tested against WP error.

* Indicates a design with $n_{1}, n_{2} ; k_{1}, k_{2} ; b_{1}, b_{2}$ and WLP identical to the design immediately preceding it in the table; the distinguishing design characteristic lies in (at least) one of the columns labeled (a) - (f). 
Table B.7 (Cont'd)

\begin{tabular}{lllcccccc}
\hline \hline$n$ & Design & WLP and Generators & (a) & (b) & (c) & (d) & (e) & (f) \\
\hline 11 & 2,$7 ; 0,5 ; 1,1$ & 4121412824024412112 & 0 & 0 & 0 & 0 & 0 & 0 \\
& & $A B \beta_{1}, p q r, A B p q s$ & & & & & & \\
& & $A B q t, B p u, A p v$ & & & & & \\
& $B p q \delta_{1}$ & & & & & & \\
& &
\end{tabular}




\section{B.8 Catalog of MA 32-Run BFFSP Designs via Mixed Blocking}


Table B.8: MA 32-Run BFFSP Designs via Mixed Blocking.

\begin{tabular}{|c|c|c|c|c|c|c|c|c|}
\hline$n$ & Design & WLP and Generators & (a) & (b) & (c) & (d) & (e) & (f) \\
\hline \multirow[t]{6}{*}{8} & 2,$4 ; 0,1 ; 1,1$ & 010312 & 6 & 14 & 4 & 14 & 0 & 1 \\
\hline & & $A B \beta_{1}, A B p q s, A p q r \delta_{1}$ & & & & & & \\
\hline & 3,$3 ; 0,1 ; 1,1$ & 001402 & 6 & 9 & 3 & 7 & 0 & 2 \\
\hline & & $A B C \beta_{1}, A B q r, A C p q \delta_{1}$ & & & & & & \\
\hline & 4,$2 ; 1,0 ; 1,1$ & 021004 & 6 & 9 & 2 & 9 & 0 & 1 \\
\hline & & $A B C D, A B \beta 1, A C p q \delta_{1}$ & & & & & & \\
\hline \multirow[t]{9}{*}{9} & 2,$4 ; 0,1 ; 1,2$ & 0308031 & 6 & 12 & 4 & 12 & 0 & 4 \\
\hline & & $A B \beta_{1}, A B p q r s, B p q \delta_{1}$ & & & & & & \\
\hline & & $B p r \delta_{2}$ & & & & & & \\
\hline & 2,$5 ; 0,2 ; 1,1$ & 0116240001 & 7 & 14 & 5 & 14 & 0 & 0 \\
\hline & & $A B \beta_{1}, p q r s, A B p q t$ & & & & & & \\
\hline & & $B p r \delta_{1}$ & & & & & & \\
\hline & 3,$3 ; 0,1 ; 2,1$ & 04061301 & 6 & 11 & 3 & 11 & 0 & 2 \\
\hline & & $A B \beta_{1}, A C \beta_{2}, A B C q r$ & & & & & & \\
\hline & & $p q \delta_{1}$ & & & & & & \\
\hline
\end{tabular}

NOTE: The $2^{\left(n_{1}+n_{2}\right)-\left(k_{1}+k_{2}\right) \pm\left(b_{1}+b_{2}\right)}$ designs are labeled as "Design $=n_{1}, n_{2} ; k_{1}, k_{2} ; b_{1}, b_{2}$ " and are ordered by the number of treatment and blocking factors, $n=n_{1}+n_{2}+b_{1}+b_{2}$.

To save space the WLPs are truncated at the last non-zero value. A-G and $\mathrm{p}-\mathrm{v}$ are used to denote the WP and SP factors, respectively. The $i^{\text {th }}$ and $j^{\text {th }}$ WP blocking variable and separator are denoted by $\beta_{i}$ and $\delta_{j}$, respectively. Finally, the last letter in each generator represents the added factor.
(a) The number of clear main effects.
(b) The number of clear two-factor interactions.
(c) The number of clear SP main effects.
(d) The number of clear SP two-factor interactions.
(e) The number of clear SP main effects tested against WP error.
(f) The number of clear SP two-factor interactions tested against WP error. 
Table B.8 (Cont'd)

\begin{tabular}{|c|c|c|c|c|c|c|c|c|}
\hline$n$ & Design & WLP and Generators & (a) & (b) & (c) & (d) & (e) & (f) \\
\hline \multirow[t]{15}{*}{9} & 3,$4 ; 0,2 ; 1,1$ & 003704000001 & 7 & 6 & 4 & 4 & 0 & 0 \\
\hline & & $A B C \beta_{1}, B C p r, B C q s$ & & & & & & \\
\hline & & $B p q \delta_{1}$ & & & & & & \\
\hline & * & * & 7 & 6 & 4 & 6 & 0 & 3 \\
\hline & & $A B C \beta_{1}, A B q r, A C q s$ & & & & & & \\
\hline & & $B C p q \delta_{1}$ & & & & & & \\
\hline & 4,$2 ; 1,0 ; 2,1$ & 06140004 & 6 & 9 & 2 & 9 & 0 & 1 \\
\hline & & $A B C D, A B \beta_{1}, A C \beta_{2}$ & & & & & & \\
\hline & & $A B C p q \delta_{1}$ & & & & & & \\
\hline & 4,$3 ; 1,1 ; 1,1$ & 021524000001 & 7 & 15 & 3 & 15 & 1 & 15 \\
\hline & & $A B C D, A B \beta_{1}, A B p q r$ & & & & & & \\
\hline & & $A C p q \delta_{1}$ & & & & & & \\
\hline & 5,$2 ; 2,0 ; 1,1$ & 221304020001 & 2 & 11 & 2 & 11 & 0 & 1 \\
\hline & & $A B C D, A B E, A C \beta_{1}$ & & & & & & \\
\hline & & $A B p q \delta_{1}$ & & & & & & \\
\hline \multirow[t]{9}{*}{10} & 2,$5 ; 0,2 ; 1,2$ & 05112260203 & 7 & 12 & 5 & 12 & 1 & 0 \\
\hline & & $A B \beta_{1}, A B q r s, p q r t$ & & & & & & \\
\hline & & $A p r \delta_{1}, p q \delta_{2}$ & & & & & & \\
\hline & * & * & 7 & 12 & 5 & 12 & 0 & 6 \\
\hline & & $A B \beta_{1}, A B p s$, Apqrt & & & & & & \\
\hline & & $A p r \delta_{1}, B p q \delta_{2}$ & & & & & & \\
\hline & 2,$6 ; 0,3 ; 1,1$ & 0131048000302 & 8 & 12 & 6 & 12 & 0 & 0 \\
\hline & & $A B \beta_{1}, p q r s, A B p q t$ & & & & & & \\
\hline & & $A B p r u, B q r \delta_{1}$ & & & & & & \\
\hline
\end{tabular}

* Indicates a design with $n_{1}, n_{2} ; k_{1}, k_{2} ; b_{1}, b_{2}$ and WLP identical to the design immediately preceding it in the table; the distinguishing design characteristic lies in (at least) one of the columns labeled (a) - (f). 
Table B.8 (Cont'd)

\begin{tabular}{|c|c|c|c|c|c|c|c|c|}
\hline$n$ & Design & WLP and Generators & (a) & (b) & (c) & (d) & (e) & (f) \\
\hline \multirow[t]{18}{*}{10} & 3,$4 ; 0,2 ; 2,1$ & 05112260203 & 7 & 12 & 4 & 12 & 0 & 0 \\
\hline & & $A B \beta_{1}, A C \beta_{2}, A B C q r$ & & & & & & \\
\hline & & $A B C p s, p q \delta_{1}$ & & & & & & \\
\hline & 3,$5 ; 0,3 ; 1,1$ & 0131048000302 & 8 & 12 & 5 & 10 & 1 & 2 \\
\hline & & $A B C \beta_{1}, A B p q r, A C p q s$ & & & & & & \\
\hline & & $B C q t, B C p \delta_{1}$ & & & & & & \\
\hline & * & $*$ & 8 & 12 & 5 & 12 & 0 & 6 \\
\hline & & $A B C \beta_{1}, B C p r, A C p s$ & & & & & & \\
\hline & & $A B C q t, C p q \delta_{1}$ & & & & & & \\
\hline & 4,$3 ; 1,1 ; 2,1$ & 0738040801 & 7 & 6 & 3 & 6 & 0 & 2 \\
\hline & & $A B C D, A B \beta_{1}, A C \beta 2$ & & & & & & \\
\hline & & $A B q r, A B C p q \delta_{1}$ & & & & & & \\
\hline & 4,$4 ; 1,2 ; 1,1$ & 02580528000001 & 8 & 4 & 4 & 4 & 0 & 4 \\
\hline & & $A B C D, A B \beta_{1}, A C q r$ & & & & & & \\
\hline & & $B C p s, A B C p q \delta_{1}$ & & & & & & \\
\hline & 5,$3 ; 2,1 ; 1,1$ & 223726040401 & 3 & 9 & 3 & 9 & 0 & 2 \\
\hline & & $A B C D, A B E, A C \beta_{1}$ & & & & & & \\
\hline & & $B C q r, A B C p q \delta_{1}$ & & & & & & \\
\hline
\end{tabular}




\section{Appendix C}

Catalog of Initial, Elevated and Separated MA BFFSP Designs 
Table C.1: 32-Run Triples: Initial Design is an MA FFSP Design

\begin{tabular}{|c|c|c|c|c|c|c|c|c|}
\hline$n$ & Design & WLP and Generators & (a) & (b) & (c) & (d) & (e) & (f) \\
\hline \multirow[t]{18}{*}{6} & 2,$4 ; 0,1 ; 0,0$ & $\begin{array}{lllllll}0 & 0 & 0 & 0 & 0 & 0 & 1\end{array}$ & 6 & 15 & 4 & 14 & 0 & 0 \\
\hline & & ABpqrs & & & & & & \\
\hline & 3,$3 ; 0,1 ; 1,0$ & 000111 & 6 & 15 & 3 & 12 & 0 & 0 \\
\hline & & $A B C \beta_{1}, A B p q r$ & & & & & & \\
\hline & 2,$4 ; 0,1 ; 0,1$ & 000111 & 6 & 15 & 4 & 14 & 0 & 1 \\
\hline & & $A B p q s, p q r \delta_{1}$ & & & & & & \\
\hline & 3,$3 ; 0,1 ; 0,0$ & 000000001 & 6 & 15 & 3 & 12 & 0 & 0 \\
\hline & & $A B C p q r$ & & & & & & \\
\hline & 4,$2 ; 0,1 ; 1,0$ & 000111 & 6 & 15 & 2 & 9 & 0 & 1 \\
\hline & & $A B C D \beta_{1}, A B C p q$ & & & & & & \\
\hline & 3,$3 ; 0,1 ; 0,1$ & 000111 & 6 & 15 & 3 & 12 & 0 & 3 \\
\hline & & $A B C q r, A C p q \delta_{1}$ & & & & & & \\
\hline & 3,$3 ; 1,0 ; 0,0$ & 1 & 3 & 12 & 3 & 12 & 0 & 0 \\
\hline & & $A B C$ & & & & & & \\
\hline & 4,$2 ; 1,0 ; 1,0$ & 021 & 6 & 9 & 2 & 9 & 0 & 0 \\
\hline & & $A B C D, A B \beta_{1}$ & & & & & & \\
\hline & 3,$3 ; 1,0 ; 0,1$ & 10000101 & 3 & 12 & 3 & 12 & 0 & 0 \\
\hline & & $A B C, A B p q r \delta_{1}$ & & & & & & \\
\hline
\end{tabular}

NOTE: The $2^{\left(n_{1}+n_{2}\right)-\left(k_{1}+k_{2}\right) \pm\left(b_{1}+b_{2}\right)}$ designs are labeled as "Design $=n_{1}, n_{2} ; k_{1}, k_{2} ; b_{1}, b_{2}$ " and are ordered by the number of treatment and blocking factors, $n=n_{1}+n_{2}+b_{1}+b_{2}$. The initial, elevated and separated designs appear first, second and third, respectively, within a triple.

To save space the WLPs are truncated at the last non-zero value. A-G and $p-w$ are used to denote the WP and SP factors, respectively. The $i^{t h}$ and $j^{\text {th }}$ WP blocking variable and separator are denoted by $\beta_{i}$ and $\delta_{j}$, respectively. Finally, the last letter in each generator represents the added factor.

(a) The number of clear main effects.

(b) The number of clear two-factor interactions.

(c) The number of clear SP main effects.

(d) The number of clear SP two-factor interactions.

(e) The number of clear SP main effects tested against WP error.

(f) The number of clear SP two-factor interactions tested against WP error. 
Table C.1 (Cont'd)

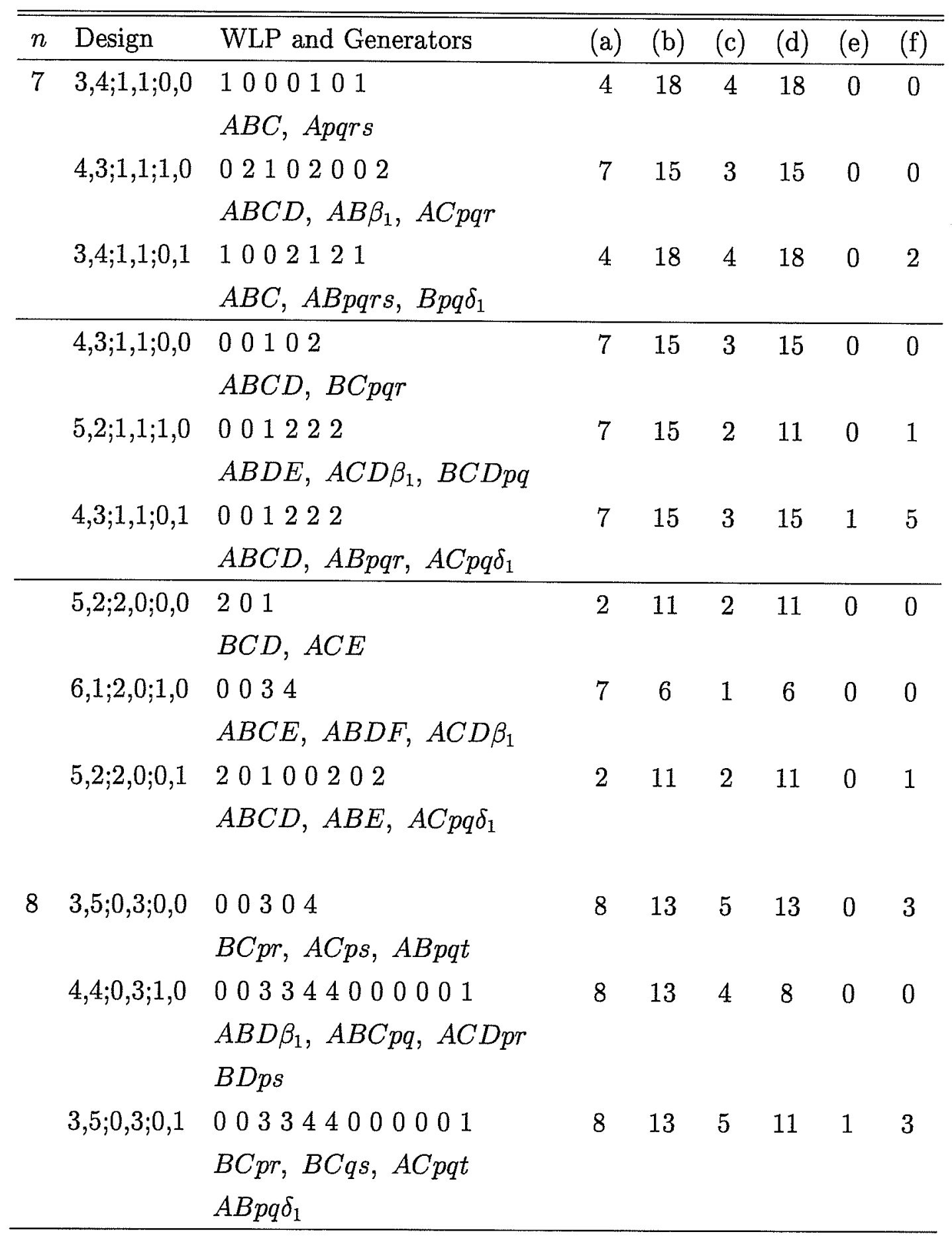


Table C.1 (Cont'd)

\begin{tabular}{|c|c|c|c|c|c|c|c|c|}
\hline$n$ & Design & WLP and Generators & (a) & (b) & (c) & (d) & (e) & (f) \\
\hline \multirow[t]{24}{*}{8} & 3,$5 ; 1,2 ; 0,0$ & 1020301 & 5 & 13 & 5 & 13 & 0 & 0 \\
\hline & & $A B C, A q r s, B p r t$ & & & & & & \\
\hline & 4,$4 ; 1,2 ; 1,0$ & 023142020001 & 8 & 13 & 4 & 13 & 0 & 0 \\
\hline & & $A B C D, A B \beta_{1}, A B p q r$ & & & & & & \\
\hline & & $A C p q s$ & & & & & & \\
\hline & 3,$5 ; 1,2 ; 0,1$ & 1023331101 & 5 & 13 & 5 & 13 & 0 & 2 \\
\hline & & $A B C, A B p q r s$, Bpqt & & & & & & \\
\hline & & $B p r \delta_{1}$ & & & & & & \\
\hline & 4,$4 ; 1,2 ; 0,0$ & 00304 & 8 & 13 & 4 & 13 & 0 & 1 \\
\hline & & $A B C D, B C p r, A C p q s$ & & & & & & \\
\hline & 5,$3 ; 1,2 ; 1,0$ & 0033440000001 & 8 & 13 & 3 & 9 & 0 & 2 \\
\hline & & $A B C E, A B D \beta_{1}, A C D p q$ & & & & & & \\
\hline & & $B C D p r$ & & & & & & \\
\hline & 4,$4 ; 1,2 ; 0,1$ & 003344000001 & 8 & 13 & 4 & 13 & 1 & 6 \\
\hline & & $A B C D, A B p q r, A C p q s$ & & & & & & \\
\hline & & $B C q \delta_{1}$ & & & & & & \\
\hline & 5,$3 ; 2,1 ; 0,0$ & 2010202 & 3 & 18 & 3 & 18 & 0 & 0 \\
\hline & & $B C D, A C E, A B p q r$ & & & & & & \\
\hline & 6,$2 ; 2,1 ; 1,0$ & 00334430000000001 & 8 & 13 & 2 & 13 & 0 & 1 \\
\hline & & $A B C E, A B D F, A C D \beta_{1}$ & & & & & & \\
\hline & & $B C D p q$ & & & & & & \\
\hline & 5,$3 ; 2,1 ; 0,1$ & 2013242000001 & 3 & 18 & 3 & 18 & 1 & 6 \\
\hline & & $A B C D, A B E, A C p q r$ & & & & & & \\
\hline & & $A B p q \delta_{1}$ & & & & & & \\
\hline
\end{tabular}


Table C.1 (Cont'd)

\begin{tabular}{|c|c|c|c|c|c|c|c|c|}
\hline$n$ & Design & WLP and Generators & (a) & (b) & (c) & (d) & (e) & (f) \\
\hline \multirow[t]{27}{*}{9} & 2,$7 ; 0,4 ; 0,0$ & 0060880000001 & 9 & 8 & 7 & 8 & 0 & 1 \\
\hline & & $A B p s$, ABqrt, Apru & & & & & & \\
\hline & & $A p q v$ & & & & & & \\
\hline & 3,$6 ; 0,4 ; 1,0$ & 006488000014 & 9 & 8 & 6 & 6 & 0 & 0 \\
\hline & & $A B C \beta_{1}, A B p q r, A C p q s$ & & & & & & \\
\hline & & $B C q t, B C p u$ & & & & & & \\
\hline & 2,$7 ; 0,4 ; 0,1$ & 0064888000014 & 9 & 8 & 7 & 8 & 0 & 2 \\
\hline & & $A B p s$, ABqrt, Apru & & & & & & \\
\hline & & $B p q r v, A p q \delta_{1}$ & & & & & & \\
\hline & 3,$6 ; 0,4 ; 0,0$ & 0060080000001 & 9 & 8 & 6 & 8 & 0 & 4 \\
\hline & & $B C p r, A C p s, A B C q t$ & & & & & & \\
\hline & & $C p q u$ & & & & & & \\
\hline & 4,$5 ; 0,4 ; 1,0$ & 0064880000014 & 9 & 8 & 5 & 8 & 0 & 4 \\
\hline & & $A B C D \beta_{1}, A B C p q, A B D p r$ & & & & & & \\
\hline & & $A C D p s, B C D p t$ & & & & & & \\
\hline & 3,$6 ; 0,4 ; 0,1$ & 006488000014 & 9 & 8 & 6 & 8 & 1 & 4 \\
\hline & & $A B p q r, A C p q s, B C p q t$ & & & & & & \\
\hline & & $A B C q u, A B C p \delta_{1}$ & & & & & & \\
\hline & 3,$6 ; 1,3 ; 0,0$ & 105060201 & 6 & 9 & 6 & 9 & 0 & 0 \\
\hline & & $A B C$, Aqrs, Aprt & & & & & & \\
\hline & & $B p q u$ & & & & & & \\
\hline & 4,$5 ; 1,3 ; 1,0$ & 026284040212 & 9 & 8 & 5 & 8 & 0 & 0 \\
\hline & & $A B C D, A B \beta_{1}, A B p q r$ & & & & & & \\
\hline & & $A C p q s, B C q t$ & & & & & & \\
\hline & 3,$6 ; 1,3 ; 0,1$ & 10556522120101 & 6 & 9 & 6 & 9 & 0 & 1 \\
\hline & & $A B C, p q r s, A B p q t$ & & & & & & \\
\hline & & $B p r u, A B q r \delta_{1}$ & & & & & & \\
\hline
\end{tabular}


Table C.1 (Cont'd)

\begin{tabular}{|c|c|c|c|c|c|c|c|c|}
\hline$n$ & Design & WLP and Generators & (a) & (b) & (c) & (d) & (e) & (f) \\
\hline \multirow[t]{25}{*}{9} & 4,$5 ; 1,3 ; 0,0$ & 0060080000001 & 9 & 8 & 5 & 8 & 0 & 2 \\
\hline & & $\begin{array}{l}A B C D, B C p r, B C q s \\
A C p q t\end{array}$ & & & & & & \\
\hline & 5,$4 ; 1,3 ; 1,0$ & 006488000014 & 9 & 8 & 4 & 4 & 0 & 0 \\
\hline & & $\begin{array}{l}A B C E, A B D \beta_{1}, A C D p q \\
B C D p r, A B p s\end{array}$ & & & & & & \\
\hline & 4,$5 ; 1,3 ; 0,1$ & 006488000014 & 9 & 8 & 5 & 8 & 1 & 4 \\
\hline & & $A B C D, A B p q r, A C p q s$ & & & & & & \\
\hline & & $B C q t, B C p \delta_{1}$ & & & & & & \\
\hline & 5,$4 ; 2,2 ; 0,0$ & 204060200001 & 4 & 11 & 4 & 11 & 0 & 1 \\
\hline & & $B C D, A C E, A B p r$ & & & & & & \\
\hline & & Cpqs & & & & & & \\
\hline & 6,$3 ; 2,2 ; 1,0$ & 009700660003 & 9 & 0 & 3 & 0 & 0 & 0 \\
\hline & & $A B C E, A B D F, A C D \beta_{1}$ & & & & & & \\
\hline & & $A B C D p q, A C p r$ & & & & & & \\
\hline & 5,$4 ; 2,2 ; 0,1$ & 204466220212 & 4 & 11 & 4 & 11 & 1 & 5 \\
\hline & & $A B C D, A B E, A B p q r$ & & & & & & \\
\hline & & $A C p q s, B C q \delta_{1}$ & & & & & & \\
\hline & 6,$3 ; 3,1 ; 0,0$ & 4030030400000001 & 3 & 21 & 3 & 21 & 0 & 0 \\
\hline & & $B C D, A C E, A B F$ & & & & & & \\
\hline & & $A B C p q r$ & & & & & & \\
\hline & 7,$2 ; 3,1 ; 1,0$ & 0010800440014 & 9 & 2 & 2 & 2 & 0 & 0 \\
\hline & & $A B C E, A B D F, A C D G$ & & & & & & \\
\hline & & $B C D \beta_{1}, A B C D p q$ & & & & & & \\
\hline & 6,$3 ; 3,1 ; 0,1$ & 404426222212 & 3 & 15 & 3 & 15 & 1 & 5 \\
\hline & & $A B C D, A B E, A C F$ & & & & & & \\
\hline & & $A B C p q r, A p q \delta_{1}$ & & & & & & \\
\hline
\end{tabular}


Table C.2: 32-Run Triples: Initial Design is an MA BFFSP Design with Pure WP Blocking

\begin{tabular}{lllcccccc}
\hline \hline$n$ & Design & WLP and Generators & (a) & (b) & (c) & (d) & (e) & (f) \\
\hline 7 & 2,$4 ; 0,1 ; 1,0$ & 0100011 & 6 & 14 & 4 & 14 & 0 & 0 \\
& $A B \beta_{1}, A B p q r s$ & & & & & & \\
3,$3 ; 0,1 ; 2,0$ & 0300031 & 6 & 12 & 3 & 12 & 0 & 0 \\
& $A B \beta_{1}, A C \beta_{2}, A B C p q r$ & & & & & & \\
2,$4 ; 0,1 ; 1,1$ & 010312 & 6 & 14 & 4 & 14 & 0 & 1 \\
& $A B \beta_{1}, A B p q s, A p q r \delta_{1}$ & & & & & & \\
\hline 3,$3 ; 0,1 ; 1,0$ & 000111 & 6 & 15 & 3 & 12 & 0 & 0 \\
& $A B C \beta_{1}, A B p q r$ & & & & & & \\
4,$2 ; 0,1 ; 2,0$ & 010312 & 6 & 14 & 2 & 9 & 0 & 1 \\
& $A B C \beta_{1}, A B D \beta_{2}, A C D p q$ & & & & & & \\
3,$3 ; 0,1 ; 1,1$ & 001402 & 6 & 9 & 3 & 7 & 0 & 2 \\
& $A B C \beta_{1}, A B q r, A C p q \delta_{1}$ & & & & & & \\
\hline
\end{tabular}

NOTE: The $2^{\left(n_{1}+n_{2}\right)-\left(k_{1}+k_{2}\right) \pm\left(b_{1}+b_{2}\right)}$ designs are labeled as "Design $=n_{1}, n_{2} ; k_{1}, k_{2} ; b_{1}, b_{2}$ " and are ordered by the number of treatment and blocking factors, $n=n_{1}+n_{2}+b_{1}+b_{2}$. The initial, elevated and separated designs appear first, second and third, respectively, within a triple.

To save space the WLPs are truncated at the last non-zero value. A-G and $\mathrm{p}-\mathrm{w}$ are used to denote the WP and SP factors, respectively. The $i^{t h}$ and $j^{t h}$ WP blocking variable and separator are denoted by $\beta_{i}$ and $\delta_{j}$, respectively. Finally, the last letter in each generator represents the added factor.
(a) The number of clear main effects.
(b) The number of clear two-factor interactions.
(c) The number of clear SP main effects.
(d) The number of clear SP two-factor interactions.
(e) The number of clear SP main effects tested against WP error.
(f) The number of clear SP two-factor interactions tested against WP error. 
Table C.2 (Cont'd)

\begin{tabular}{|c|c|c|c|c|c|c|c|c|}
\hline$n$ & Design & WLP and Generators & (a) & (b) & (c) & (d) & (e) & $(\mathrm{f})$ \\
\hline \multirow[t]{24}{*}{8} & 3,$3 ; 0,1 ; 2,0$ & 0300031 & 6 & 12 & 3 & 12 & 0 & 0 \\
\hline & & $A B \beta_{1}, A C \beta_{2}, A B C p q r$ & & & & & & \\
\hline & 4,$2 ; 0,1 ; 3,0$ & 06041103 & 6 & 9 & 2 & 9 & 0 & 1 \\
\hline & & $A B \beta_{1}, A C \beta_{2}, A D \beta_{3}$ & & & & & & \\
\hline & & $A B C p q$ & & & & & & \\
\hline & 3,$3 ; 0,1 ; 2,1$ & 04061301 & 6 & 11 & 3 & 11 & 0 & 2 \\
\hline & & $A B \beta_{1}, A C \beta_{2}, A B C q r$ & & & & & & \\
\hline & & $p q \delta_{1}$ & & & & & & \\
\hline & 3,$4 ; 0,2 ; 1,0$ & 001222 & 7 & 15 & 4 & 13 & 0 & 0 \\
\hline & & $A B C \beta_{1}, A B p q r, A C p q s$ & & & & & & \\
\hline & 4,$3 ; 0,2 ; 2,0$ & 0116240001 & 7 & 14 & 3 & 10 & 0 & 2 \\
\hline & & $A B C \beta_{1}, A B D \beta_{2}, A C D p q$ & & & & & & \\
\hline & & $B C D p r$ & & & & & & \\
\hline & 3,$4 ; 0,2 ; 1,1$ & 003704000001 & 7 & 6 & 4 & 4 & 0 & 0 \\
\hline & & $A B C \beta_{1}, B C p r, B C q s$ & & & & & & \\
\hline & & $B p q \delta_{1}$ & & & & & & \\
\hline & 4,$3 ; 1,1 ; 1,0$ & 02102002 & 7 & 15 & 3 & 15 & 0 & 0 \\
\hline & & $A B C D, A B \beta_{1}, A C p q r$ & & & & & & \\
\hline & 5,$2 ; 1,1 ; 2,0$ & 021524000001 & 7 & 15 & 2 & 11 & 0 & 1 \\
\hline & & $A B C E, A B D \beta_{1}, A C D \beta_{2}$ & & & & & & \\
\hline & & $B C D p q$ & & & & & & \\
\hline & 4,$3 ; 1,1 ; 1,1$ & 0215240000001 & 7 & 15 & 3 & 15 & 1 & 15 \\
\hline & & $A B C D, A B \beta_{1}, A B p q r$ & & & & & & \\
\hline & & $A C p q \delta_{1}$ & & & & & & \\
\hline
\end{tabular}


Table C.2 (Cont'd)

\begin{tabular}{|c|c|c|c|c|c|c|c|c|}
\hline$n$ & Design & WLP and Generators & (a) & (b) & (c) & (d) & (e) & (f) \\
\hline & 5,$2 ; 2,0 ; 1,0$ & 2212 & 2 & 11 & 2 & 11 & 0 & 0 \\
\hline 8 & & $B C D, A C E, A B \beta_{1}$ & & & & & & \\
\hline & 6,$1 ; 2,0 ; 2,0$ & 03380000001 & 7 & 6 & 1 & 6 & 0 & 0 \\
\hline & & $A B C E, A B D F, A C D \beta_{1}$ & & & & & & \\
\hline & & $B C D \beta_{2}$ & & & & & & \\
\hline & 5,$2 ; 2,0 ; 1,1$ & 221304020001 & 2 & 11 & 2 & 11 & 0 & 1 \\
\hline & & $A B C D, A B E, A C \beta_{1}$ & & & & & & \\
\hline & & $A B p q \delta_{1}$ & & & & & & \\
\hline \multirow[t]{18}{*}{9} & 3,$5 ; 0,3 ; 1,0$ & 00334400000001 & 8 & 13 & 5 & 11 & 0 & 0 \\
\hline & & $A B C \beta_{1}, A B p q r, A C p q s$ & & & & & & \\
\hline & & $B C q t$ & & & & & & \\
\hline & 4,$4 ; 0,3 ; 2,0$ & 0131048000302 & 8 & 12 & 4 & 8 & 0 & 0 \\
\hline & & $A B C \beta_{1}, A B D \beta_{2}, A C D p q$ & & & & & & \\
\hline & & $B C D p r, A B p s$ & & & & & & \\
\hline & 3,$5 ; 0,3 ; 1,1$ & 0131048000302 & 8 & 12 & 5 & 10 & 1 & 2 \\
\hline & & $A B C \beta_{1}, A B p q r, A C p q s$ & & & & & & \\
\hline & & $B C q t, B C p \delta_{1}$ & & & & & & \\
\hline & 4,$3 ; 1,1 ; 2,0$ & 061120040001 & 7 & 15 & 3 & 15 & 0 & 0 \\
\hline & & $A B C D, A B \beta_{1}, A C \beta_{2}$ & & & & & & \\
\hline & & $A B p q r$ & & & & & & \\
\hline & 5,$2 ; 1,1 ; 3,0$ & 0101524080001 & 7 & 11 & 2 & 11 & 0 & 1 \\
\hline & & $A B C E, A B C D \beta_{1}, A B \beta_{2}$ & & & & & & \\
\hline & & $A D \beta_{3}, A B D p q$ & & & & & & \\
\hline & 4,$3 ; 1,1 ; 2,1$ & 0738040801 & 7 & 6 & 3 & 6 & 0 & 2 \\
\hline & & $A B C D, A B \beta_{1}, A C \beta 2$ & & & & & & \\
\hline & & $A B q r, A B C p q \delta_{1}$ & & & & & & \\
\hline
\end{tabular}


Table C.2 (Cont'd)

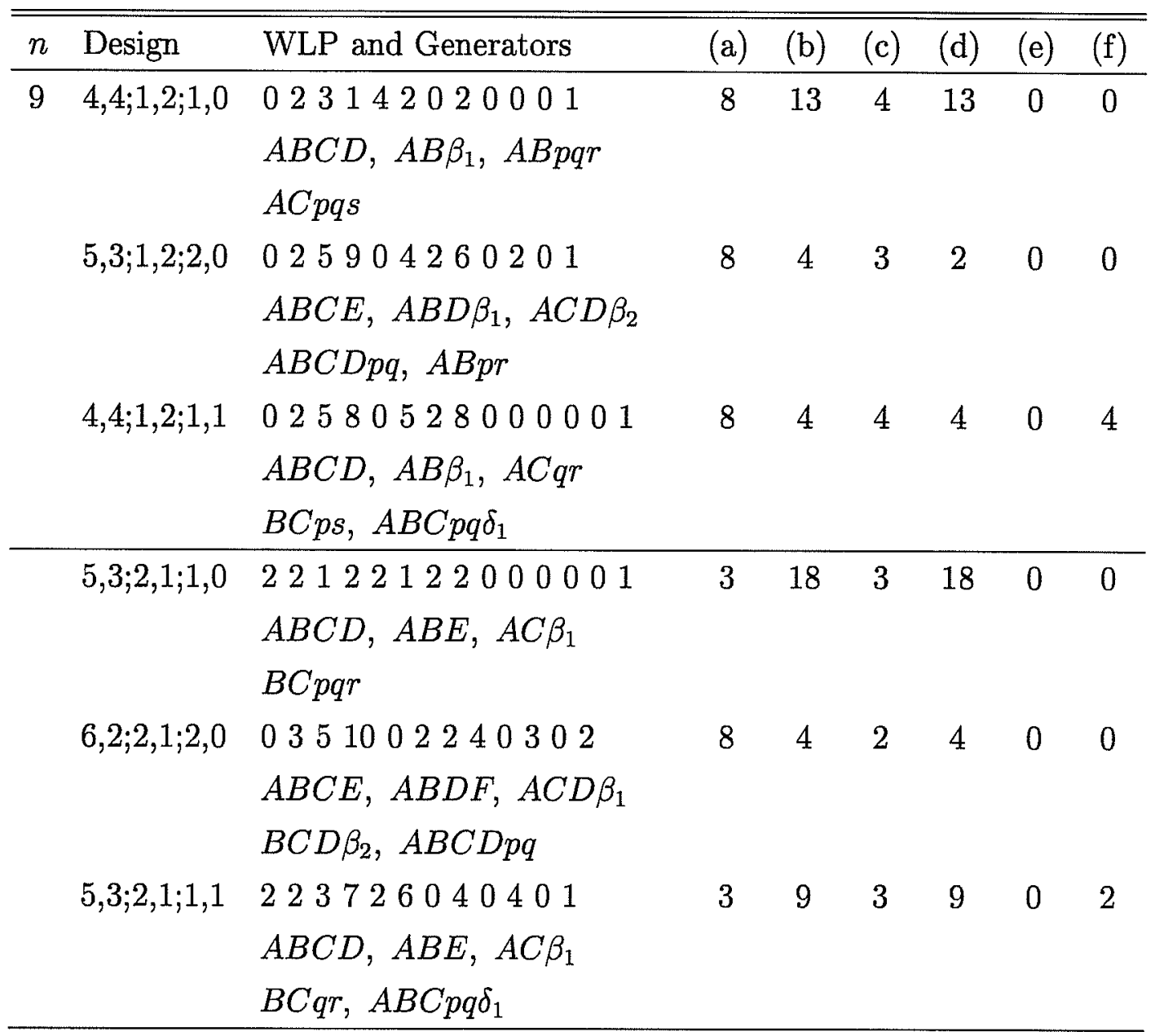


Table C.3: 32-Run Triples: Initial Design is an MA BFFSP Design with Separation

\begin{tabular}{lllcccccc}
\hline \hline$n$ & Design & WLP and Generators & (a) & (b) & (c) & (d) & (e) & (f) \\
\hline 7 & 1,$5 ; 0,1 ; 0,1$ & 000111 & 6 & 15 & 5 & 15 & 0 & 0 \\
& Apqrt, pqs $\delta_{1}$ & & & & & & \\
2,$4 ; 0,1 ; 1,1$ & 010312 & 6 & 14 & 4 & 14 & 0 & 1 \\
& $A B \beta_{1}, A B p q s, A p q r \delta_{1}$ & & & & & & \\
1,$5 ; 0,1 ; 0,2$ & 001402 & 6 & 9 & 5 & 9 & 0 & 0 \\
& $p q r t, p q s \delta_{1}, A p r s \delta_{2}$ & & & & & & \\
\hline 3,$3 ; 1,0 ; 0,1$ & 10000101 & 3 & 12 & 3 & 12 & 0 & 0 \\
& $A B C, A B p q r \delta_{1}$ & & & & & & \\
4,$2 ; 1,0 ; 1,1$ & 021004 & 6 & 9 & 2 & 9 & 0 & 1 \\
& $A B C D, A B \beta 1, A C p q \delta_{1}$ & & & & & & \\
3,$3 ; 1,0 ; 0,2$ & 100303 & 3 & 12 & 3 & 12 & 0 & 3 \\
& $A B C, A B p q \delta_{1}, B p r \delta_{2}$ & & & & & & \\
\hline
\end{tabular}

NOTE: The $2^{\left(n_{1}+n_{2}\right)-\left(k_{1}+k_{2}\right) \pm\left(b_{1}+b_{2}\right)}$ designs are labeled as "Design $=n_{1}, n_{2} ; k_{1}, k_{2} ; b_{1}, b_{2}$ " and are ordered by the number of treatment and blocking factors, $n=n_{1}+n_{2}+b_{1}+b_{2}$. The initial, elevated and separated designs appear first, second and third, respectively, within a triple.

To save space the WLPs are truncated at the last non-zero value. A-G and p-w are used to denote the WP and SP factors, respectively. The $i^{\text {th }}$ and $j^{t h}$ WP blocking variable and separator are denoted by $\beta_{i}$ and $\delta_{j}$, respectively. Finally, the last letter in each generator represents the added factor.
(a) The number of clear main effects.
(b) The number of clear two-factor interactions.
(c) The number of clear SP main effects.
(d) The number of clear SP two-factor interactions.
(e) The number of clear SP main effects tested against WP error.
(f) The number of clear SP two-factor interactions tested against WP error. 
Table C.3 (Cont'd)

\begin{tabular}{|c|c|c|c|c|c|c|c|c|}
\hline$n$ & Design & WLP and Generators & (a) & (b) & (c) & (d) & (e) & $(f)$ \\
\hline \multirow[t]{24}{*}{8} & 1,$5 ; 0,1 ; 0,2$ & 001402 & 6 & 9 & 5 & 9 & 0 & 0 \\
\hline & & $p q r t, p q s \delta_{1}, A p r s \delta_{2}$ & & & & & & \\
\hline & 2,$4 ; 0,1 ; 1,2$ & 0308031 & 6 & 12 & 4 & 12 & 0 & 4 \\
\hline & & $A B \beta_{1}, A B p q r s, B p q \delta_{1}$ & & & & & & \\
\hline & & $B p r \delta_{2}$ & & & & & & \\
\hline & 1,$5 ; 0,1 ; 0,3$ & 0308031 & 6 & 12 & 5 & 12 & 1 & 4 \\
\hline & & $A p q r s t, A p q \delta_{1}, A r \delta_{2}$ & & & & & & \\
\hline & & $\mathrm{Aps}_{3}$ & & & & & & \\
\hline & 1,$6 ; 0,2 ; 0,1$ & 001222 & 7 & 15 & 6 & 15 & 0 & 0 \\
\hline & & $p q r t$, Aprsu, qrs $\delta_{1}$ & & & & & & \\
\hline & 2,$5 ; 0,2 ; 1,1$ & 0116240001 & 7 & 14 & 5 & 14 & 0 & 0 \\
\hline & & $A B \beta_{1}, p q r s, A B p q t$ & & & & & & \\
\hline & & $B p r \delta_{1}$ & & & & & & \\
\hline & 1,$6 ; 0,2 ; 0,2$ & 0037040000001 & 7 & 6 & 6 & 6 & 0 & 0 \\
\hline & & $p q r t, p q s u, p r s \delta_{1}$ & & & & & & \\
\hline & & $A q r s \delta_{2}$ & & & & & & \\
\hline & 2,$5 ; 0,2 ; 0,1$ & 001222 & 7 & 15 & 5 & 14 & 0 & 0 \\
\hline & & $p q r s, A B p q t, A B p r \delta_{1}$ & & & & & & \\
\hline & 3,$4 ; 0,2 ; 1,1$ & 00337040000001 & 7 & 6 & 4 & 4 & 0 & 0 \\
\hline & & $A B C \beta_{1}, B C p r, B C q s$ & & & & & & \\
\hline & & $B p q \delta_{1}$ & & & & & & \\
\hline & 2,$5 ; 0,2 ; 0,2$ & 0037040000001 & 7 & 6 & 5 & 6 & 1 & 2 \\
\hline & & $A B r s, A B q t, B q r \delta_{1}$ & & & & & & \\
\hline & & $A B p \delta_{2}$ & & & & & & \\
\hline
\end{tabular}


Table C.3 (Cont'd)

\begin{tabular}{|c|c|c|c|c|c|c|c|c|}
\hline$n$ & Design & WLP and Generators & (a) & (b) & (c) & (d) & (e) & (f) \\
\hline \multirow[t]{8}{*}{8} & 3,$4 ; 1,1 ; 0,1$ & 1002121 & 4 & 18 & 4 & 18 & 0 & 2 \\
\hline & & $A B C, A B p q r s, B p q \delta_{1}$ & & & & & & \\
\hline & 4,$3 ; 1,1 ; 1,1$ & 02152400000001 & 7 & 15 & 3 & 15 & 1 & 15 \\
\hline & & $A B C D, A B \beta_{1}, A B p q r$ & & & & & & \\
\hline & & $A C p q \delta_{1}$ & & & & & & \\
\hline & 3,$4 ; 1,1 ; 0,2$ & 101606001 & 4 & 12 & 4 & 12 & 0 & 0 \\
\hline & & $A B C, p q r s, A B p q \delta_{1}$ & & & & & & \\
\hline & & $B p r \delta_{2}$ & & & & & & \\
\hline \multirow[t]{18}{*}{9} & 1,$6 ; 0,2 ; 0,2$ & 00370400000001 & 7 & 6 & 6 & 6 & 0 & 0 \\
\hline & & pqrt, pqsu, prs $\delta_{1}$ & & & & & & \\
\hline & & $A q r s \delta_{2}$ & & & & & & \\
\hline & 2,$5 ; 0,2 ; 1,2$ & 05112260203 & 7 & 12 & 5 & 12 & 1 & 0 \\
\hline & & $A B \beta_{1}, A B q r s$, pqrt & & & & & & \\
\hline & & $A p r \delta_{1}, p q \delta_{2}$ & & & & & & \\
\hline & 1,$6 ; 0,2 ; 0,3$ & 05112260203 & 7 & 12 & 6 & 12 & 2 & 0 \\
\hline & & Aqrst, Aprsu, Apqs $\delta_{1}$ & & & & & & \\
\hline & & $r s \delta_{2}, A r \delta_{3}$ & & & & & & \\
\hline & 1,$7 ; 0,3 ; 0,1$ & 00333440000001 & 8 & 32 & 7 & 28 & 0 & 0 \\
\hline & & pqrst, Apqru, Apqsv & & & & & & \\
\hline & & $\operatorname{Aprs}_{1}$ & & & & & & \\
\hline & 2,$6 ; 0,3 ; 1,1$ & 0131048000302 & 8 & 12 & 6 & 12 & 0 & 0 \\
\hline & & $A B \beta_{1}, p q r s, A B p q t$ & & & & & & \\
\hline & & $A B p r u, B q r \delta_{1}$ & & & & & & \\
\hline & 1,$7 ; 0,3 ; 0,2$ & 0131048000302 & 8 & 12 & 7 & 12 & 1 & 0 \\
\hline & & Aqrst, $p r s u$, Apqsv & & & & & & \\
\hline & & $A q \delta_{1}, A p r \delta_{2}$ & & & & & & \\
\hline
\end{tabular}


Table C.3 (Cont'd)

\begin{tabular}{lllcccccc}
\hline \hline$n$ & Design & WLP and Generators & (a) & (b) & (c) & (d) & (e) & (f) \\
\hline 9 & 3,$5 ; 1,2 ; 0,1$ & 1023331101 & 5 & 13 & 5 & 13 & 0 & 2 \\
& & $A B C, A B p q r s, B p q t$ & & & & & & \\
& $B p r \delta_{1}$ & & & & & & \\
4,$4 ; 1,2 ; 1,1$ & 02580528000001 & 8 & 4 & 4 & 4 & 0 & 4 \\
& $A B C D, A B \beta_{1}, A C q r$ & & & & & & \\
& $B C p s, A B C p q \delta_{1}$ & & & & & & \\
3,$5 ; 1,2 ; 0,2$ & 113928021301 & 5 & 9 & 5 & 9 & 1 & 1 \\
& $A B C, p q r s, A B p q t$ & & & & & & \\
& $B p q \delta_{1}, A B p r \delta_{2}$ & & & & & & \\
\hline
\end{tabular}


Table C.4: 32-Run Triples: Initial Design is an MA BFFSP Design with Mixed Blocking

\begin{tabular}{|c|c|c|c|c|c|c|c|c|}
\hline$n$ & Design & WLP and Generators & (a) & (b) & (c) & (d) & (e) & (f) \\
\hline \multirow[t]{8}{*}{8} & 2,$4 ; 0,1 ; 1,1$ & 010312 & 6 & 14 & 4 & 14 & 0 & 1 \\
\hline & & $A B \beta_{1}, A B p q s, A p q r \delta_{1}$ & & & & & & \\
\hline & 3,$3 ; 0,1 ; 2,1$ & 04061301 & 6 & 11 & 3 & 11 & 0 & 2 \\
\hline & & $A B \beta_{1}, A C \beta_{2}, A B C q r$ & & & & & & \\
\hline & & $p q \delta_{1}$ & & & & & & \\
\hline & 2,$4 ; 0,1 ; 1,2$ & 0308031 & 6 & 12 & 4 & 12 & 0 & 4 \\
\hline & & $A B \beta_{1}, A B p q r s, B p q \delta_{1}$ & & & & & & \\
\hline & & $B p r \delta_{2}$ & & & & & & \\
\hline \multirow[t]{9}{*}{9} & 2,$5 ; 0,2 ; 1,1$ & 0116240001 & 7 & 14 & 5 & 14 & 0 & 0 \\
\hline & & $A B \beta_{1}, p q r s, A B p q t$ & & & & & & \\
\hline & & $B p r \delta_{1}$ & & & & & & \\
\hline & 3,$4 ; 0,2 ; 2,1$ & 05112260203 & 7 & 12 & 4 & 12 & 0 & 0 \\
\hline & & $A B \beta_{1}, A C \beta_{2}, A B C q r$ & & & & & & \\
\hline & & $A B C p s, p q \delta_{1}$ & & & & & & \\
\hline & 2,$5 ; 0,2 ; 1,2$ & 05112260203 & 7 & 12 & 5 & 12 & 1 & 0 \\
\hline & & $A B \beta_{1}, A B q r s, p q r t$ & & & & & & \\
\hline & & $A p r \delta_{1}, p q \delta_{2}$ & & & & & & \\
\hline
\end{tabular}

NOTE: The $2^{\left(n_{1}+n_{2}\right)-\left(k_{1}+k_{2}\right) \pm\left(b_{1}+b_{2}\right)}$ designs are labeled as "Design $=n_{1}, n_{2} ; k_{1}, k_{2} ; b_{1}, b_{2}$ " and are ordered by the number of treatment and blocking factors, $n=n_{1}+n_{2}+b_{1}+b_{2}$. The initial, elevated and separated designs appear first, second and third, respectively, within a triple.

To save space the WLPs are truncated at the last non-zero value. A-G and $\mathrm{p}-\mathrm{w}$ are used to denote the WP and SP factors, respectively. The $i^{t h}$ and $j^{t h}$ WP blocking variable and separator are denoted by $\beta_{i}$ and $\delta_{j}$, respectively. Finally, the last letter in each generator represents the added factor.

(a) The number of clear main effects.

(b) The number of clear two-factor interactions.

(c) The number of clear SP main effects.

(d) The number of clear SP two-factor interactions.

(e) The number of clear SP main effects tested against WP error.

(f) The number of clear SP two-factor interactions tested against WP error. 


\section{Appendix D}

Catalog of Initial and Elevated

MA BFFSP Designs 
Table D.1: 32-Run Pairs: Elevation of an MA Initial Design with Pure WP Blocking to an MA Design with Pure WP Blocking

\begin{tabular}{|c|c|c|c|c|c|c|c|c|}
\hline$n$ & Design & WLP and Generators & (a) & (b) & (c) & (d) & (e) & (f) \\
\hline \multirow[t]{4}{*}{7} & 3,$3 ; 0,1 ; 1,0$ & 000111 & 6 & 15 & 3 & 12 & 0 & 0 \\
\hline & & $A B C \beta_{1}, A B p q r$ & & & & & & \\
\hline & 2,$4 ; 0,1 ; 1,0$ & 0100011 & 6 & 14 & 4 & 14 & 0 & 0 \\
\hline & & $A B \beta_{1}, A B p q r s$ & & & & & & \\
\hline \multirow[t]{8}{*}{8} & 3,$4 ; 0,2 ; 1,0$ & 001222 & 7 & 15 & 4 & 13 & 0 & 0 \\
\hline & & $A B C \beta_{1}, A B p q r, A C p q s$ & & & & & & \\
\hline & 2,$5 ; 0,2 ; 1,0$ & 01112101 & 7 & 14 & 5 & 14 & 0 & 0 \\
\hline & & $A B \beta_{1}, B p q r s, A B p q t$ & & & & & & \\
\hline & 4,$2 ; 0,1 ; 2,0$ & 010312 & 6 & 14 & 2 & 9 & 0 & 1 \\
\hline & & $A B C \beta_{1}, A B D \beta_{2}, A C D p q$ & & & & & & \\
\hline & 3,$3 ; 0,1 ; 2,0$ & 0300031 & 6 & 12 & 3 & 12 & 0 & 0 \\
\hline & & $A B \beta_{1}, A C \beta_{2}, A B C p q r$ & & & & & & \\
\hline
\end{tabular}

NOTE: The $2^{\left(n_{1}+n_{2}\right)-\left(k_{1}+k_{2}\right) \pm\left(b_{1}+b_{2}\right)}$ designs are labeled as "Design $=n_{1}, n_{2} ; k_{1}, k_{2} ; b_{1}, b_{2}$ " and are ordered by the number of treatment and blocking factors, $n=n_{1}+n_{2}+b_{1}+b_{2}$. The elevated and initial designs appear first and second, respectively, within a pair.

To save space the WLPs are truncated at the last non-zero value. A-G and $p-v$ are used to denote the WP and SP factors, respectively. The $i^{\text {th }}$ WP blocking variable is denoted by $\beta_{i}$. Finally, the last letter in each generator represents the added factor.

(a) The number of clear main effects.

(b) The number of clear two-factor interactions.

(c) The number of clear SP main effects.

(d) The number of clear SP two-factor interactions.

(e) The number of clear SP main effects tested against WP error.

(f) The number of clear SP two-factor interactions tested against WP error. 
Table D.1 (Cont'd)

\begin{tabular}{|c|c|c|c|c|c|c|c|c|}
\hline$n$ & Design & WLP and Generators & (a) & (b) & (c) & (d) & (e) & (f) \\
\hline \multirow[t]{18}{*}{9} & 3,$5 ; 0,3 ; 1,0$ & 003344000001 & 8 & 13 & 5 & 11 & 0 & 0 \\
\hline & & $A B C \beta_{1}, A B p q r, A C p q s$ & & & & & & \\
\hline & & $B C q t$ & & & & & & \\
\hline & 2,$6 ; 0,3 ; 1,0$ & 0132420201 & 8 & 12 & 6 & 12 & 0 & 0 \\
\hline & & $A B \beta_{1}, p q r s, A B p q t$ & & & & & & \\
\hline & & Bpru & & & & & & \\
\hline & 4,$3 ; 0,2 ; 2,0$ & 0116240001 & 7 & 14 & 3 & 10 & 0 & 2 \\
\hline & & $A B C \beta_{1}, A B D \beta_{2}, A C D p q$ & & & & & & \\
\hline & & $B C D p r$ & & & & & & \\
\hline & 3,$4 ; 0,2 ; 2,0$ & 0314220201 & 7 & 12 & 4 & 12 & 0 & 1 \\
\hline & & $A B \beta_{1}, A C \beta_{2}, A B p q r$ & & & & & & \\
\hline & & Cpqs & & & & & & \\
\hline & 6,$2 ; 2,1 ; 1,0$ & 00344300000001 & 8 & 13 & 2 & 13 & 0 & 1 \\
\hline & & $A B C E, A B D F, A C D \beta_{1}$ & & & & & & \\
\hline & & $B C D p q$ & & & & & & \\
\hline & 5,$3 ; 2,1 ; 1,0$ & 2212212220000001 & 3 & 18 & 3 & 18 & 0 & 0 \\
\hline & & $A B C D, A B E, A C \beta_{1}$ & & & & & & \\
\hline & & $B C p q r$ & & & & & & \\
\hline \multirow[t]{6}{*}{10} & 3,$6 ; 0,4 ; 1,0$ & 006488000014 & 9 & 8 & 6 & 6 & 0 & 0 \\
\hline & & $A B C \beta_{1}, A B p q r, A C p q s$ & & & & & & \\
\hline & & $B C q t, B C p u$ & & & & & & \\
\hline & 2,$7 ; 0,4 ; 1,0$ & 01648304031001 & 9 & 7 & 7 & 7 & 0 & 0 \\
\hline & & $A B \beta_{1}, p q r s, A B p q t$ & & & & & & \\
\hline & & $A B p r u, B q r v$ & & & & & & \\
\hline
\end{tabular}


Table D.1 (Cont'd)

\begin{tabular}{|c|c|c|c|c|c|c|c|c|}
\hline$n$ & Design & WLP and Generators & (a) & (b) & (c) & (d) & (e) & (f) \\
\hline \multirow[t]{23}{*}{10} & 4,$4 ; 0,3 ; 2,0$ & 0131048000302 & 8 & 12 & 4 & 8 & 0 & 0 \\
\hline & & $\begin{array}{l}A B C \beta_{1}, A B D \beta_{2}, A C D p q \\
B C D p r, A B p s\end{array}$ & & & & & & \\
\hline & 3,$5 ; 0,3 ; 2,0$ & 0338440405 & 8 & 10 & 5 & 10 & 0 & 0 \\
\hline & & $A B \beta_{1}, A C \beta_{2}, A B p q r$ & & & & & & \\
\hline & & $C p q s, A B C q t$ & & & & & & \\
\hline & 4,$5 ; 0,4 ; 1,0$ & 006488000014 & 9 & 8 & 5 & 8 & 0 & 4 \\
\hline & & $A B C D \beta_{1}, A B C p q, A B D p r$ & & & & & & \\
\hline & & $A C D p s, B C D p t$ & & & & & & \\
\hline & 3,$6 ; 0,4 ; 1,0$ & 006488000014 & 9 & 8 & 6 & 6 & 0 & 0 \\
\hline & & $A B C \beta_{1}, A B p q r, A C p q s$ & & & & & & \\
\hline & & $B C q t, B C p u$ & & & & & & \\
\hline & 6,$3 ; 2,2 ; 1,0$ & 009700660003 & 9 & 0 & 3 & 0 & 0 & 0 \\
\hline & & $A B C E, A B D F, A C D \beta_{1}$ & & & & & & \\
\hline & & $A B C D p q, A C p r$ & & & & & & \\
\hline & 5,$4 ; 2,2 ; 1,0$ & 224462220412 & 4 & 11 & 4 & 11 & 0 & 0 \\
\hline & & $A B C D, A B E, A C \beta_{1}$ & & & & & & \\
\hline & & $A B p q r, A C p q s$ & & & & & & \\
\hline & 7,$2 ; 3,1 ; 1,0$ & 0010800440014 & 9 & 2 & 2 & 2 & 0 & 0 \\
\hline & & $A B C E, A B D F, A C D G$ & & & & & & \\
\hline & & $B C D \beta_{1}, A B C D p q$ & & & & & & \\
\hline & 6,$3 ; 3,1 ; 1,0$ & 4335304005031 & 3 & 21 & 3 & 21 & 0 & 0 \\
\hline & & $A B C D, A B E, A C F$ & & & & & & \\
\hline & & $B C \beta_{1}, B C p q r$ & & & & & & \\
\hline
\end{tabular}


Table D.2: 32-Run Pairs: Elevation of a Separated MA Initial Design to a Separated MA Design

\begin{tabular}{|c|c|c|c|c|c|c|c|c|}
\hline$n$ & Design & WLP and Generators & (a) & (b) & (c) & (d) & (e) & (f) \\
\hline \multirow[t]{4}{*}{7} & 4,$2 ; 1,0 ; 0,1$ & 001002 & 6 & 9 & 2 & 9 & 0 & 1 \\
\hline & & $A B C D, A B p q \delta_{1}$ & & & & & & \\
\hline & 3,$3 ; 1,0 ; 0,1$ & 10000101 & 3 & 12 & 3 & 12 & 0 & 0 \\
\hline & & $A B C, A B p q r \delta_{1}$ & & & & & & \\
\hline \multirow[t]{4}{*}{8} & 4,$3 ; 1,1 ; 0,1$ & 001222 & 7 & 15 & 3 & 15 & 1 & 5 \\
\hline & & $A B C D, A B p q r, A C p q \delta_{1}$ & & & & & & \\
\hline & 3,$4 ; 1,1 ; 0,1$ & 1002121 & 4 & 18 & 4 & 18 & 0 & 2 \\
\hline & & $A B C, A B p q r s, B p q \delta_{1}$ & & & & & & \\
\hline \multirow[t]{6}{*}{9} & 4,$4 ; 1,2 ; 0,1$ & 003344000001 & 8 & 13 & 4 & 13 & 1 & 6 \\
\hline & & $A B C D, A B p q r, A C p q s$ & & & & & & \\
\hline & & $B C q \delta_{1}$ & & & & & & \\
\hline & 3,$5 ; 1,2 ; 0,1$ & 1023331101 & 5 & 13 & 5 & 13 & 0 & 2 \\
\hline & & $A B C, A B p q r s, B p q t$ & & & & & & \\
\hline & & $B p r \delta_{1}$ & & & & & & \\
\hline
\end{tabular}

NOTE: The $2^{\left(n_{1}+n_{2}\right)-\left(k_{1}+k_{2}\right) \pm\left(b_{1}+b_{2}\right)}$ designs are labeled as "Design $=n_{1}, n_{2} ; k_{1}, k_{2} ; b_{1}, b_{2}$ " and are ordered by the number of treatment and blocking factors, $n=n_{1}+n_{2}+b_{1}+b_{2}$. The elevated and initial designs appear first and second, respectively, within a pair.

To save space the WLPs are truncated at the last non-zero value. A-G and p-v are used to denote the WP and SP factors, respectively. The $j^{\text {th }}$ separator is denoted by $\delta_{j}$. Finally, the last letter in each generator represents the added factor.
(a) The number of clear main effects.
(b) The number of clear two-factor interactions.
(c) The number of clear SP main effects.
(d) The number of clear SP two-factor interactions.
(e) The number of clear SP main effects tested against WP error.
(f) The number of clear SP two-factor interactions tested against WP error. 
Table D.2 (Cont'd)

\begin{tabular}{lllcccccc}
\hline \hline$n$ & Design & WLP and Generators & (a) & (b) & (c) & (d) & (e) & (f) \\
\hline 10 & 2,$6 ; 0,3 ; 0,2$ & 0131048000302 & 8 & 12 & 6 & 12 & 0 & 0 \\
& & $p q r s, A B p q t, A B p r u$ & & & & & & \\
& $B q r \delta_{1}, A q r \delta_{2}$ & & & & & & \\
& & & & & & & \\
1,$7 ; 0,3 ; 0,2$ & 0131048000302 & 8 & 12 & 7 & 12 & 1 & 0 \\
& $A q r s t, p r s u, A p q s v$ & & & & & & \\
& $A q \delta_{1}, A p r \delta_{2}$ & & & & & & \\
\hline 4,$5 ; 1,3 ; 0,1$ & 006488000014 & 9 & 8 & 5 & 8 & 1 & 4 \\
& $A B C D, A B p q r, A C p q s$ & & & & & & \\
& $B C q t, B C p \delta_{1}$ & & & & & & \\
3,$6 ; 1,3 ; 0,1$ & 10556522120101 & 6 & 9 & 6 & 9 & 0 & 1 \\
& $A B C, p q r s, A B p q t$ & & & & & \\
& $B p r u, A B q r \delta_{1}$ & & & & & \\
\hline
\end{tabular}


Table D.3: 32-Run Pairs: Elevation of a Separated MA Initial Design to an MA Design with Pure WP Blocking

\begin{tabular}{|c|c|c|c|c|c|c|c|c|}
\hline$n$ & Design & WLP and Generators & (a) & (b) & (c) & (d) & (e) & (f) \\
\hline \multirow[t]{8}{*}{8} & 3,$3 ; 0,1 ; 2,0$ & 0300031 & 6 & 12 & 3 & 12 & 0 & 0 \\
\hline & & $A B \beta_{1}, A C \beta_{2}, A B C p q r$ & & & & & & \\
\hline & 2,$4 ; 0,1 ; 0,2$ & 001402 & 6 & 9 & 4 & 8 & 0 & 0 \\
\hline & & $p q r s, A B p q \delta_{1}, B p r \delta_{2}$ & & & & & & \\
\hline & 4,$2 ; 1,0 ; 2,0$ & 061 & 6 & 9 & 2 & 9 & 0 & 0 \\
\hline & & $A B C D, A B \beta_{1}, A C \beta_{2}$ & & & & & & \\
\hline & 3,$3 ; 1,0 ; 0,2$ & 100303 & 3 & 12 & 3 & 12 & 0 & 3 \\
\hline & & $A B C, A B p q \delta_{1}, B p r \delta_{2}$ & & & & & & \\
\hline \multirow[t]{6}{*}{9} & 3,$4 ; 0,2 ; 2,0$ & 0314220201 & 7 & 12 & 4 & 12 & 0 & 1 \\
\hline & & $A B \beta_{1}, A C \beta_{2}, A B p q r$ & & & & & & \\
\hline & & $C p q s$ & & & & & & \\
\hline & 2,$5 ; 0,2 ; 0,2$ & 003704000001 & 7 & 6 & 5 & 6 & 1 & 2 \\
\hline & & $A B r s, A B q t, B q r \delta_{1}$ & & & & & & \\
\hline & & $A B p \delta_{2}$ & & & & & & \\
\hline
\end{tabular}

NOTE: The $2^{\left(n_{1}+n_{2}\right)-\left(k_{1}+k_{2}\right) \pm\left(b_{1}+b_{2}\right)}$ designs are labeled as "Design $=n_{1}, n_{2} ; k_{1}, k_{2} ; b_{1}, b_{2}$ " and are ordered by the number of treatment and blocking factors, $n=n_{1}+n_{2}+b_{1}+b_{2}$. The elevated and initial designs appear first and second, respectively, within a pair.

To save space the WLPs are truncated at the last non-zero value. A-G and $\mathrm{p}-\mathrm{v}$ are used to denote the WP and SP factors, respectively. The $i^{t h}$ and $j^{t h}$ WP blocking variable and separator are denoted by $\beta_{i}$ and $\delta_{j}$, respectively. Finally, the last letter in each generator represents the added factor.

(a) The number of clear main effects.

(b) The number of clear two-factor interactions.

(c) The number of clear SP main effects.

(d) The number of clear SP two-factor interactions.

(e) The number of clear SP main effects tested against WP error.

(f) The number of clear SP two-factor interactions tested against WP error. 
Table D.3 (Cont'd)

\begin{tabular}{|c|c|c|c|c|c|c|c|c|}
\hline$n$ & Design & WLP and Generators & (a) & (b) & (c) & (d) & (e) & (f) \\
\hline \multirow[t]{5}{*}{9} & 4,$3 ; 1,1 ; 2,0$ & 061120040001 & 7 & 15 & 3 & 15 & 0 & 0 \\
\hline & & $\begin{array}{l}A B C D, A B \beta_{1}, A C \beta_{2} \\
A B p q r\end{array}$ & & & & & & \\
\hline & 3,$4 ; 1,1 ; 0,2$ & 101606001 & 4 & 12 & 4 & 12 & 0 & 0 \\
\hline & & $A B C, p q r s, A B p q \delta_{1}$ & & & & & & \\
\hline & & $B p r \delta_{2}$ & & & & & & \\
\hline \multirow[t]{6}{*}{10} & 4,$4 ; 1,2 ; 2,0$ & 073244080102 & 8 & 13 & 4 & 13 & 0 & 0 \\
\hline & & $A B C D, A B \beta_{1}, A C \beta_{2}$ & & & & & & \\
\hline & & $A B p q r, A C p q s$ & & & & & & \\
\hline & 3,$5 ; 1,2 ; 0,2$ & 113928021301 & 5 & 9 & 5 & 9 & 1 & 1 \\
\hline & & $A B C, p q r s, A B p q t$ & & & & & & \\
\hline & & $B p q \delta_{1}, A B p r \delta_{2}$ & & & & & & \\
\hline
\end{tabular}


Table D.4: 32-Run Pairs: Elevation of an MA Initial Design with Pure WP Blocking to a Separated MA Design

\begin{tabular}{|c|c|c|c|c|c|c|c|c|}
\hline$n$ & Design & WLP and Generators & (a) & (b) & (c) & (d) & (e) & (f) \\
\hline \multirow[t]{4}{*}{7} & 3,$3 ; 0,1 ; 0,1$ & 000111 & 6 & 15 & 3 & 12 & 0 & 3 \\
\hline & & $A B C q r, A C p q \delta_{1}$ & & & & & & \\
\hline & 2,$4 ; 0,1 ; 1,0$ & 0100011 & 6 & 14 & 4 & 14 & 0 & 0 \\
\hline & & $A B \beta_{1}, A B p q r s$ & & & & & & \\
\hline \multirow[t]{4}{*}{8} & 3,$4 ; 0,2 ; 0,1$ & 001222 & 7 & 15 & 4 & 12 & 0 & 0 \\
\hline & & $A B C p r, A B C q s, A B p q \delta_{1}$ & & & & & & \\
\hline & 2,$5 ; 0,2 ; 1,0$ & 01112101 & 7 & 14 & 5 & 14 & 0 & 0 \\
\hline & & $A B \beta_{1}$, Bpqrs, $A B p q t$ & & & & & & \\
\hline \multirow[t]{6}{*}{9} & 3,$5 ; 0,3 ; 0,1$ & 0033440000001 & 8 & 13 & 5 & 11 & 1 & 3 \\
\hline & & $B C p r, B C q s, A C p q t$ & & & & & & \\
\hline & & $A B p q \delta_{1}$ & & & & & & \\
\hline & 2,$6 ; 0,3 ; 1,0$ & 0132420201 & 8 & 12 & 6 & 12 & 0 & 0 \\
\hline & & $A B \beta_{1}, p q r s, A B p q t$ & & & & & & \\
\hline & & Bpru & & & & & & \\
\hline
\end{tabular}

NOTE: The $2^{\left(n_{1}+n_{2}\right)-\left(k_{1}+k_{2}\right) \pm\left(b_{1}+b_{2}\right)}$ designs are labeled as "Design $=n_{1}, n_{2} ; k_{1}, k_{2} ; b_{1}, b_{2}$ " and are ordered by the number of treatment and blocking factors, $n=n_{1}+n_{2}+b_{1}+b_{2}$. The elevated and initial designs appear first and second, respectively, within a pair.

To save space the WLPs are truncated at the last non-zero value. A-G and $p-v$ are used to denote the WP and SP factors, respectively. The $i^{t h}$ and $j^{\text {th }}$ WP blocking variable and separator are denoted by $\beta_{i}$ and $\delta_{j}$, respectively. Finally, the last letter in each generator represents the added factor.
(a) The number of clear main effects.
(b) The number of clear two-factor interactions.
(c) The number of clear SP main effects.
(d) The number of clear SP two-factor interactions.
(e) The number of clear SP main effects tested against WP error.
(f) The number of clear SP two-factor interactions tested against WP error. 
Table D.4 (Cont'd)

\begin{tabular}{lllcccccc}
\hline \hline$n$ & Design & WLP and Generators & (a) & (b) & (c) & (d) & (e) & (f) \\
\hline 10 & 3,$6 ; 0,4 ; 0,1$ & 006488000014 & 9 & 8 & 6 & 8 & 1 & 4 \\
& & $A B p q r, A C p q s, B C p q t$ & & & & & & \\
& $A B C q u, A B C p \delta_{1}$ & & & & & & \\
\multirow{2}{*}{2,$7 ; 0,4 ; 1,0$} & 01648304031001 & 9 & 7 & 7 & 7 & 0 & 0 \\
& & $A B \beta_{1}$, pqrs, $A B p q t$ & & & & & & \\
& & $A B p r u$, Bqrv & & & & & & \\
\hline
\end{tabular}


Appendix E

Catalog of Optimal BFFSP Robust Parameter Designs 
E.1 Catalog of Optimal 16-Run BFFSP RPDs via Pure WP Blocking: Control Factors as SP Factors 
Table E.1: Optimal 16-Run BFFSP RPDs via Pure WP Blocking: Control Factors as SP Factors

\begin{tabular}{|c|c|c|c|c|c|c|c|c|c|}
\hline$n$ & $\overline{\text { Design }}$ & Design Generators & (a) & (b) & (c) & $\overline{(d)}$ & (e) & (f) & $\overline{(\mathrm{g})}$ \\
\hline \multirow[t]{3}{*}{6} & 2,$3 ; 0,1 ; 1,0$ & $A B \beta_{1}, A B p q r$ & 3 & 6 & 3 & 0 & 0 & 0 & 9 \\
\hline & 3,$2 ; 0,1 ; 1,0$ & $B C \beta_{1}, A B C p q$ & 2 & 6 & 1 & 0 & 0 & 1 & 8 \\
\hline & 4,$1 ; 1,0 ; 1,0$ & $B C D, B C \beta_{1}$ & 1 & 4 & 0 & 0 & 0 & 0 & 5 \\
\hline \multirow[t]{8}{*}{7} & 2,$4 ; 0,2 ; 1,0$ & $A B \beta_{1}, A B p r, A B q s$ & 4 & 0 & 0 & 0 & 0 & 0 & 4 \\
\hline & * & $A B \beta_{1}, A B p q r, A B p q s$ & & & & & & & 6 \\
\hline & 3,$2 ; 0,1 ; 2,0$ & $B C \beta_{1}, B C \beta_{2}, A B C p q$ & 2 & 6 & 1 & 0 & 0 & 1 & 8 \\
\hline & 3,$3 ; 0,2 ; 1,0$ & $B C \beta_{1}, B C p q, A C p r$ & 3 & 0 & 0 & 0 & 0 & 0 & 3 \\
\hline & * & $B C \beta_{1}, A p q, B C p r$ & & & & & & & 4 \\
\hline & 3,$3 ; 1,1 ; 1,0$ & $A B C, A B \beta_{1}, A p q r$ & 3 & 6 & 0 & 0 & 0 & 0 & 9 \\
\hline & 4,$2 ; 1,1 ; 1,0$ & $B C D, B C \beta_{1}, A C p q$ & 2 & 4 & 0 & 0 & 0 & 0 & 6 \\
\hline & 4,$1 ; 1,0 ; 2,0$ & $B C D, B C \beta_{1}, B C \beta_{1}$ & 1 & 4 & 0 & 0 & 0 & 0 & 5 \\
\hline
\end{tabular}

NOTE: The $2^{\left(n_{1}+n_{2}\right)-\left(k_{1}+k_{2}\right) \pm\left(b_{1}+b_{2}\right)}$ designs are labeled as "Design $=n_{1}, n_{2} ; k_{1}, k_{2} ; b_{1}, b_{2}$ " and are ordered by the number of treatment and blocking factors, $n=n_{1}+n_{2}+b_{1}+b_{2}$.

A-G and $\mathrm{p}-\mathrm{v}$ are used to denote the WP and SP factors, respectively. The $i^{\text {th }}$ WP blocking variable is denoted by $\beta_{i}$. Finally, the last letter in each generator represents the added factor.

(a) The number of clear $\mathrm{C}$ main effects.

(b) The number of clear CN two-factor interactions.

(c) The number of clear CC two-factor interactions.

(d) The number of clear $\mathrm{C}$ main effects tested against WP error.

(e) The number of clear CN two-factor interactions tested against WP error.

(f) The number of clear CC two-factor interactions tested against WP error.

(g) One number optimality criterion: Total number of clear $\mathrm{C}$ main effects and $\mathrm{CN}$ two-factor interactions.

* Indicates a design that is optimal with respect to the one-number criterion in the event that the design immediately preceding it in the table is not. 
Table E.1 (Cont'd)

\begin{tabular}{|c|c|c|c|c|c|c|c|c|c|}
\hline$n$ & Design & Design Generators & (a) & (b) & (c) & (d) & (e) & (f) & 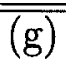 \\
\hline \multirow[t]{8}{*}{8} & 2,$5 ; 0,3 ; 1,0$ & $\begin{array}{l}A B \beta_{1}, A B p r, A B q s \\
A p q t\end{array}$ & 5 & 0 & 0 & 0 & 0 & 0 & 5 \\
\hline & * & $\begin{array}{l}A B \beta_{1}, A B p q r, A B p q s \\
A B p q t\end{array}$ & & & & & & & 6 \\
\hline & 3,$3 ; 0,2 ; 2,0$ & $\begin{array}{l}B C \beta_{1}, B C \beta_{2}, B C p q \\
A C p r\end{array}$ & 3 & 0 & 0 & 0 & 0 & 0 & 3 \\
\hline & * & $\begin{array}{l}B C \beta_{1}, B C \beta_{2}, A p q \\
B C p r\end{array}$ & & & & & & & 4 \\
\hline & 3,$4 ; 0,3 ; 1,0$ & $\begin{array}{l}B C \beta_{1}, B C p q, A C p r \\
A B p s\end{array}$ & 4 & 0 & 0 & 0 & 0 & 0 & 4 \\
\hline & 4,$2 ; 1,1 ; 2,0$ & $\begin{array}{l}B C D, B C \beta_{1}, B C \beta_{2} \\
A C p q\end{array}$ & 2 & 4 & 0 & 0 & 0 & 0 & 6 \\
\hline & 4,$3 ; 1,2 ; 1,0$ & $\begin{array}{l}A B C D, B C \beta_{1}, B C p q \\
A C p r\end{array}$ & 3 & 0 & 0 & 0 & 0 & 0 & 3 \\
\hline & 5,$2 ; 2,1 ; 1,0$ & $\begin{array}{l}B C D, B C E, B C \beta_{1} \\
A B C p q\end{array}$ & 2 & 4 & 0 & 0 & 0 & 0 & 6 \\
\hline \multirow[t]{5}{*}{9} & 2,$6 ; 0,4 ; 1,0$ & $\begin{array}{l}A B \beta_{1}, A B p r, A B q s \\
A p q t, B p q\end{array}$ & 6 & 0 & 0 & 0 & 0 & 0 & 6 \\
\hline & 3,$4 ; 0,3 ; 2,0$ & $\begin{array}{l}B C \beta_{1}, B C \beta_{2}, B C p q \\
A C p r, A B p s\end{array}$ & 4 & 0 & 0 & 0 & 0 & 0 & 4 \\
\hline & 3,$5 ; 0,4 ; 1,0$ & $\begin{array}{l}B C \beta_{1}, B C p q, B C p r \\
A C p s, A B p t\end{array}$ & 3 & 0 & 0 & 0 & 0 & 0 & 3 \\
\hline & * & $\begin{array}{l}B C \beta_{1}, A p q, A p r \\
\text { Aps, ABCpt }\end{array}$ & & & & & & & 4 \\
\hline & 3,$5 ; 1,3 ; 1,0$ & $\begin{array}{l}A B C, A B \beta_{1}, A p q r \\
A p q s, A p q t\end{array}$ & 2 & 4 & 0 & 0 & 0 & 0 & 6 \\
\hline
\end{tabular}


Table E.1 (Cont'd)

\begin{tabular}{|c|c|c|c|c|c|c|c|c|c|}
\hline$n$ & Design & Design Generators & (a) & (b) & (c) & (d) & (e) & (f) & $\overline{\overline{(g)}}$ \\
\hline \multirow[t]{5}{*}{9} & 4,$3 ; 1,2 ; 2,0$ & $\begin{array}{l}A B C D, B C \beta_{1}, B C \beta_{2} \\
B C p q, A C p r\end{array}$ & 3 & 0 & 0 & 0 & 0 & 0 & 3 \\
\hline & 4,$4 ; 1,3 ; 1,0$ & $\begin{array}{l}A B C D, B C \beta_{1}, B C p q \\
A C p r, A B p s\end{array}$ & 4 & 0 & 0 & 0 & 0 & 0 & 4 \\
\hline & 5,$2 ; 2,1 ; 2,0$ & $\begin{array}{l}B C D, B C E, B C \beta_{1} \\
B C \beta_{2}, A B C p q\end{array}$ & 2 & 4 & 0 & 0 & 0 & 0 & 6 \\
\hline & 5,$3 ; 2,2 ; 1,0$ & $\begin{array}{l}A B C D, A B C E, B C \beta_{1} \\
B C p q, A C p r\end{array}$ & 2 & 4 & 0 & 0 & 0 & 0 & 6 \\
\hline & 6,$2 ; 3,1 ; 1,0$ & $\begin{array}{l}B C D, B C E, B C F \\
B C \beta_{1}, A B C p q\end{array}$ & 2 & 4 & 0 & 0 & 0 & 0 & 6 \\
\hline
\end{tabular}


E.2 Catalog of Optimal 32-Run BFFSP RPDs via Pure WP Blocking: Control Factors as SP Factors 
Table E.2: Optimal 32-Run BFFSP RPDs via Pure WP Blocking: Control Factors as SP Factors

\begin{tabular}{|c|c|c|c|c|c|c|c|c|c|}
\hline$n$ & $\overline{\text { Design }}$ & Design Generators & (a) & (b) & (c) & (d) & (e) & 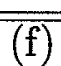 & $\overline{(\mathrm{g})}$ \\
\hline \multirow[t]{5}{*}{7} & 2,$4 ; 0,1 ; 1,0$ & $A B \beta_{1}, A B q r s$ & 4 & 8 & 6 & 0 & 0 & 0 & 12 \\
\hline & 3,$3 ; 0,1 ; 1,0$ & $B C \beta_{1}, B C p q r$ & 3 & 9 & 3 & 0 & 0 & 0 & 12 \\
\hline & 3,$3 ; 1,0 ; 1,0$ & $A B C, A B \beta_{1}$ & 3 & 9 & 3 & 0 & 0 & 0 & 12 \\
\hline & 4,$2 ; 0,1 ; 1,0$ & $C D \beta_{1}, B C D p q$ & 2 & 8 & 1 & 0 & 0 & 1 & 10 \\
\hline & 4,$2 ; 1,0 ; 1,0$ & $B C D, B C \beta_{1}$ & 2 & 8 & 1 & 0 & 0 & 0 & 10 \\
\hline \multirow[t]{6}{*}{8} & 2,$5 ; 0,2 ; 1,0$ & $A B \beta_{1}, A B q r s, A B p r t$ & 5 & 10 & 4 & 0 & 0 & 0 & 15 \\
\hline & 3,$3 ; 0,1 ; 2,0$ & $B C \beta_{1}, B C \beta_{2}, B C p q r$ & 3 & 9 & 3 & 0 & 0 & 0 & 12 \\
\hline & 3,$4 ; 0,2 ; 1,0$ & $B C \beta_{1}, A B C p r, A B C q s$ & 4 & 12 & 0 & 0 & 0 & 0 & 16 \\
\hline & 3,$4 ; 1,1 ; 1,0$ & $A B C, A B \beta_{1}$, Apqrs & 4 & 12 & 6 & 0 & 0 & 0 & 16 \\
\hline & 4,$2 ; 0,1 ; 2,0$ & $C D \beta_{1}, C D \beta_{2}, B C D p q$ & 2 & 8 & 1 & 0 & 0 & 1 & 10 \\
\hline & 4,$2 ; 1,0 ; 2,0$ & $B C D, B C \beta_{1}, B C \beta_{2}$ & 2 & 8 & 1 & 0 & 0 & 0 & 10 \\
\hline
\end{tabular}

NOTE: The $2^{\left(n_{1}+n_{2}\right)-\left(k_{1}+k_{2}\right) \pm\left(b_{1}+b_{2}\right)}$ designs are labeled as "Design $=n_{1}, n_{2} ; k_{1}, k_{2} ; b_{1}, b_{2}$ " and are ordered by the number of treatment and blocking factors, $n=n_{1}+n_{2}+b_{1}+b_{2}$.

A-G and $\mathrm{p}-\mathrm{v}$ are used to denote the WP and SP factors, respectively. The $i^{\text {th }}$ WP blocking variable is denoted by $\beta_{i}$. Finally, the last letter in each generator represents the added factor.

(a) The number of clear $\mathrm{C}$ main effects.

(b) The number of clear CN two-factor interactions.

(c) The number of clear CC two-factor interactions.

(d) The number of clear $\mathrm{C}$ main effects tested against WP error.

(e) The number of clear CN two-factor interactions tested against WP error.

(f) The number of clear CC two-factor interactions tested against WP error.

(g) One number optimality criterion: Total number of clear $\mathrm{C}$ main effects and $\mathrm{CN}$ two-factor interactions.

* Indicates a design that is optimal with respect to the one-number criterion in the event that the design immediately preceding it in the table is not. 
Table E.2 (Cont'd)

\begin{tabular}{|c|c|c|c|c|c|c|c|c|c|}
\hline$n$ & Design & Design Generators & (a) & (b) & (c) & (d) & (e) & (f) & $\overline{(\mathrm{g})}$ \\
\hline$\overline{8}$ & 4,$3 ; 0,2 ; 1,0$ & $C D \beta_{1}, C D p q, A B D p r$ & 3 & 8 & 2 & 0 & 0 & 2 & 11 \\
\hline & 4,$3 ; 1,1 ; 1,0$ & $B C D, B C \beta_{1}, A C p q r$ & 3 & 12 & 3 & 0 & 0 & 0 & 15 \\
\hline & 4,$3 ; 2,0 ; 1,0$ & $A B C, A B D, A B \beta_{1}$ & 3 & 6 & 3 & 0 & 0 & 0 & 9 \\
\hline & 5,$2 ; 1,1 ; 1,0$ & $C D E, C D \beta_{1}, A B D p q$ & 2 & 10 & 1 & 0 & 0 & 1 & 12 \\
\hline & 6,$1 ; 2,0 ; 1,0$ & $C D E, B D F, C D \beta_{1}$ & 1 & 6 & 0 & 0 & 0 & 0 & 7 \\
\hline \multirow[t]{9}{*}{9} & 2,$6 ; 0,3 ; 1,0$ & $\begin{array}{l}A B \beta_{1}, A B q r s, A B p r t \\
A B p q u\end{array}$ & 6 & 12 & 0 & 0 & 0 & 0 & 18 \\
\hline & 3,$4 ; 0,2 ; 2,0$ & $\begin{array}{l}B C \beta_{1}, B C \beta_{2}, A B C p r \\
A B C q s\end{array}$ & 4 & 12 & 0 & 0 & 0 & 0 & 16 \\
\hline & 3,$5 ; 0,3 ; 1,0$ & $\begin{array}{l}B C \beta_{1}, A B C p r, A B C q s \\
p q t\end{array}$ & 5 & 10 & 0 & 0 & 0 & 0 & 15 \\
\hline & 3,$5 ; 1,2 ; 1,0$ & $\begin{array}{l}A B C, A B \beta_{1}, \text { Aqrs } \\
\text { Aprt }\end{array}$ & 5 & 10 & 0 & 0 & 0 & 0 & 15 \\
\hline & 4,$3 ; 0,2 ; 2,0$ & $\begin{array}{l}C D \beta_{1}, C D \beta_{2}, C D p q \\
A B D p r\end{array}$ & 3 & 8 & 2 & 0 & 0 & 2 & 11 \\
\hline & 4,$3 ; 1,1 ; 2,0$ & $\begin{array}{l}B C D, B C \beta_{1}, B C \beta_{2} \\
A C p q r\end{array}$ & 3 & 12 & 3 & 0 & 0 & 0 & 15 \\
\hline & 4,$4 ; 0,3 ; 1,0$ & $\begin{array}{l}C D \beta_{1}, C D p q, A B D p r \\
A B C p s\end{array}$ & 4 & 8 & 0 & 0 & 0 & 0 & 12 \\
\hline & 4,$4 ; 1,2 ; 1,0$ & $\begin{array}{l}B C D, B C \beta_{1}, A C p r \\
B p q s\end{array}$ & 4 & 9 & 2 & 0 & 0 & 0 & 13 \\
\hline & 4,$4 ; 2,1 ; 1,0$ & $\begin{array}{l}A B C, A B D, A B \beta_{1} \\
\text { Apqrs }\end{array}$ & 4 & 8 & 6 & 0 & 0 & 0 & 12 \\
\hline
\end{tabular}


Table E.2 (Cont'd)

\begin{tabular}{|c|c|c|c|c|c|c|c|c|c|}
\hline$n$ & Design & Design Generators & (a) & (b) & (c) & (d) & (e) & (f) & (g) \\
\hline \multirow[t]{7}{*}{9} & 5,$2 ; 1,1 ; 2,0$ & $\begin{array}{l}C D E, C D \beta_{1}, C D \beta_{2} \\
A B D p q\end{array}$ & 2 & 10 & 1 & 0 & 0 & 1 & 12 \\
\hline & 5,$3 ; 1,2 ; 1,0$ & $\begin{array}{l}B C D E, C D \beta_{1}, C D p q \\
A B D p r\end{array}$ & 3 & 7 & 2 & 0 & 0 & 2 & 10 \\
\hline & 5,$1 ; 2,0 ; 2,0$ & $\begin{array}{l}B C D, A C E, B C \beta_{1} \\
B C \beta_{2}\end{array}$ & 1 & 5 & 0 & 0 & 0 & 0 & 6 \\
\hline & 5,$2 ; 2,0 ; 2,0$ & $\begin{array}{l}B C D, A C E, B C \beta_{1} \\
B C \beta_{2}\end{array}$ & 2 & 10 & 1 & 0 & 0 & 0 & 12 \\
\hline & 5,$3 ; 2,1 ; 1,0$ & $\begin{array}{l}B C D, A C E, B C \beta_{1} \\
A B p q r\end{array}$ & 3 & 15 & 3 & 0 & 0 & 0 & 18 \\
\hline & 6,$1 ; 2,0 ; 2,0$ & $\begin{array}{l}C D E, B D F, C D \beta_{1} \\
C D \beta_{2}\end{array}$ & 1 & 6 & 0 & 0 & 0 & 0 & 7 \\
\hline & 6,$2 ; 2,1 ; 1,0$ & $\begin{array}{l}C D E, B D F, C D \beta_{1} \\
A B C p q\end{array}$ & 2 & 12 & 1 & 0 & 0 & 1 & 14 \\
\hline
\end{tabular}


E.3 Catalog of Optimal 16-Run BFFSP RPDs via Pure WP Blocking: Control Factors as WP Factors 
Table E.3: Optimal 16-Run BFFSP RPDs via Pure WP Blocking: Control Factors as WP Factors

\begin{tabular}{|c|c|c|c|c|c|c|c|}
\hline$n$ & Design & Design Generators & (a) & (b) & (c) & (e) & $(\mathrm{g})$ \\
\hline \multirow[t]{3}{*}{6} & 2,$3 ; 0,1 ; 1,0$ & $A B \beta_{1}, A B p q r$ & 2 & 6 & 0 & 0 & 8 \\
\hline & 3,$2 ; 0,1 ; 1,0$ & $A B C \beta_{1}, A B C p q$ & 3 & 6 & 3 & 0 & 9 \\
\hline & 4,$1 ; 1,0 ; 1,0$ & $A B C D, B C \beta_{1}$ & 4 & 4 & 0 & 0 & 8 \\
\hline \multirow[t]{7}{*}{7} & 2,$4 ; 0,2 ; 1,0$ & $A B \beta_{1}, A B p r, A B p q s$ & 2 & 4 & 0 & 0 & 6 \\
\hline & 3,$2 ; 0,1 ; 2,0$ & $A B C \beta_{1}, A B C \beta_{2}, A B C p q$ & 3 & 6 & 3 & 0 & 9 \\
\hline & 3,$3 ; 0,2 ; 1,0$ & $A B C \beta_{1}, A B C p q, A B C p r$ & 3 & 3 & 3 & 0 & 6 \\
\hline & 3,$3 ; 1,1 ; 1,0$ & $A B C, A B \beta_{1}, p q r$ & 3 & 6 & 3 & 0 & 9 \\
\hline & 4,$2 ; 1,1 ; 1,0$ & $A B C D, B C \beta_{1}, B C p q$ & 4 & 0 & 0 & 0 & 4 \\
\hline & * & $A B C D, B C \beta_{1}, A p q$ & & & & & 9 \\
\hline & 4,$1 ; 1,0 ; 2,0$ & $A B C D, B C \beta_{1}, B C \beta_{2}$ & 4 & 4 & 0 & 0 & 8 \\
\hline \multirow[t]{2}{*}{8} & 2,$5 ; 0,3 ; 1,0$ & $A B \beta_{1}, A B p r, A B p s, A B p q t$ & 2 & 4 & 0 & 0 & 6 \\
\hline & 3,$3 ; 0,2 ; 2,0$ & $A B C \beta_{1}, A B C \beta_{2}, A B C p q, A B C p r$ & 3 & 3 & 3 & 0 & 6 \\
\hline
\end{tabular}

NOTE: The $2^{\left(n_{1}+n_{2}\right)-\left(k_{1}+k_{2}\right) \pm\left(b_{1}+b_{2}\right)}$ designs are labeled as "Design $=n_{1}, n_{2} ; k_{1}, k_{2} ; b_{1}, b_{2}$ " and are ordered by the number of treatment and blocking factors, $n=n_{1}+n_{2}+b_{1}+b_{2}$.

A-G and $\mathrm{p}-\mathrm{v}$ are used to denote the WP and SP factors, respectively. The $i^{\text {th }}$ WP blocking variable is denoted by $\beta_{i}$. Finally, the last letter in each generator represents the added factor.

(a) The number of clear $\mathrm{C}$ main effects.

(b) The number of clear $\mathrm{CN}$ two-factor interactions.

(c) The number of clear CC two-factor interactions.

(e) The number of clear CN two-factor interactions tested against WP error.

(g) One number optimality criterion: Total number of clear $\mathrm{C}$ main effects and $\mathrm{CN}$ two-factor interactions.

* Indicates a design that is optimal with respect to the one-number criterion in the event that the design immediately preceding it in the table is not. 
Table E.3 (Cont'd)

\begin{tabular}{|c|c|c|c|c|c|c|c|}
\hline$n$ & Design & Design Generators & (a) & (b) & (c) & (e) & 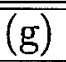 \\
\hline 8 & 3,$4 ; 0,3 ; 1,0$ & $A B C \beta_{1}, A B C p q, A B C p r, A B C p s$ & 3 & 3 & 3 & 0 & 6 \\
\hline & 4,$2 ; 1,1 ; 2,0$ & $A B C D, B C \beta_{1}, B C \beta_{2}, B C p q$ & 4 & 0 & 0 & 0 & 4 \\
\hline & * & $A B C D, B C \beta_{1}, B C \beta_{2}, A p q$ & & & & & 9 \\
\hline & 4,$3 ; 1,2 ; 1,0$ & $A B C D, B C \beta_{1}, B C p q, B C p r$ & 4 & 0 & 0 & 0 & 4 \\
\hline & * & $A B C D, B C \beta_{1}, A P q, A p r$ & & & & & 6 \\
\hline & 5,$2 ; 2,1 ; 1,0$ & $A B C D, A B C E, B C \beta_{1}, A B C p q$ & 3 & 6 & 0 & 0 & 9 \\
\hline \multirow[t]{9}{*}{9} & 2,$6 ; 0,4 ; 1,0$ & $\begin{array}{l}A B \beta_{1}, A B p r, A B p s, A B p t \\
A B p q u\end{array}$ & 2 & 4 & 0 & 0 & 6 \\
\hline & * & $\begin{array}{l}A B \beta_{1}, A p r, A q s, A p q t \\
p q u\end{array}$ & & & & & 7 \\
\hline & 3,$4 ; 0,3 ; 2,0$ & $\begin{array}{l}A B C \beta_{1}, A B C \beta_{2}, A B C p q, A B C p r \\
A B C p s\end{array}$ & 3 & 3 & 3 & 0 & 6 \\
\hline & 3,$5 ; 0,4 ; 1,0$ & $\begin{array}{l}A B C \beta_{1}, A B C p q, A B C p r, A B C p s \\
A B C p t\end{array}$ & 3 & 3 & 3 & 0 & 6 \\
\hline & 3,$5 ; 1,3 ; 1,0$ & $\begin{array}{l}A B C, A B \beta_{1}, p q r, p q s \\
p q t\end{array}$ & 0 & 6 & 0 & 0 & 6 \\
\hline & 4,$3 ; 1,2 ; 2,0$ & $\begin{array}{l}A B C D, B C \beta_{1}, B C \beta_{2}, B C p q \\
B C p r\end{array}$ & 4 & 0 & 0 & 0 & 4 \\
\hline & * & $\begin{array}{l}A B C D, B C \beta_{1}, B C \beta_{2}, A p q \\
A p r\end{array}$ & & & & & 6 \\
\hline & 4,$4 ; 1,3 ; 1,0$ & $\begin{array}{l}A B C D, B C \beta_{1}, B C p q, B C p r \\
B C p s\end{array}$ & 4 & 0 & 0 & 0 & 4 \\
\hline & * & $\begin{array}{l}A B C D, B C \beta_{1}, A p q, A p r \\
\text { Aps }\end{array}$ & & & & & 6 \\
\hline
\end{tabular}


Table E.3 (Cont'd)

\begin{tabular}{|c|c|c|c|c|c|c|c|}
\hline$n$ & Design & Design Generators & (a) & (b) & (c) & (e) & (g) \\
\hline \multirow[t]{3}{*}{9} & 5,$2 ; 2,1 ; 2,0$ & $\begin{array}{l}A B C D, A B C E, B C \beta_{1}, B C \beta_{2} \\
A B C p q\end{array}$ & 3 & 6 & 0 & 0 & 9 \\
\hline & 5,$3 ; 2,2 ; 1,0$ & $\begin{array}{l}A B C D, A B C E, B C \beta_{1}, A B C p q \\
A B C p r\end{array}$ & 3 & 3 & 0 & 0 & 6 \\
\hline & 6,$2 ; 3,1 ; 1,0$ & $\begin{array}{l}A B C D, A B C E, A B C F, B C \beta_{1} \\
A B C p q\end{array}$ & 3 & 6 & 0 & 0 & 9 \\
\hline
\end{tabular}




\section{E.4 Catalog of Optimal 32-Run BFFSP RPDs via} Pure WP Blocking: Control Factors as WP Factors 
Table E.4: Optimal 32-Run BFFSP RPDs via Pure WP Blocking: Control Factors as WP Factors

\begin{tabular}{|c|c|c|c|c|c|c|c|}
\hline$n$ & Design & Design Generators & (a) & (b) & (c) & (e) & $(\mathrm{g})$ \\
\hline \multirow[t]{5}{*}{7} & 2,$4 ; 0,1 ; 1,0$ & $A B \beta_{1}, A B q r s$ & 2 & 8 & 0 & 0 & 10 \\
\hline & 3,$3 ; 0,1 ; 1,0$ & $A B C \beta_{1}, A B C p r$ & 3 & 9 & 3 & 0 & 12 \\
\hline & 3,$3 ; 1,0 ; 1,0$ & $A B C, A B \beta_{1}$ & 0 & 9 & 0 & 0 & 9 \\
\hline & 4,$2 ; 0,1 ; 1,0$ & $B C D \beta_{1}, B C D p q$ & 4 & 8 & 6 & 0 & 12 \\
\hline & 4,$2 ; 1,0 ; 1,0$ & $A B C D, B C \beta_{1}$ & 4 & 8 & 0 & 0 & 12 \\
\hline \multirow[t]{7}{*}{8} & 2,$5 ; 0,2 ; 1,0$ & $A B \beta_{1}, A B q r s, A B p r t$ & 2 & 10 & 0 & 0 & 12 \\
\hline & 3,$3 ; 0,1 ; 2,0$ & $A B C \beta_{1}, A B C \beta_{2}, A B C p r$ & 3 & 9 & 3 & 0 & 12 \\
\hline & 3,$4 ; 0,2 ; 1,0$ & $A B C \beta_{1}, A B C p r, A B C q s$ & 3 & 12 & 3 & 0 & 15 \\
\hline & 3,$4 ; 1,1 ; 1,0$ & $A B C, A B \beta_{1}$, Apqrs & 0 & 12 & 0 & 0 & 12 \\
\hline & 4,$2 ; 0,1 ; 2,0$ & $B C D \beta_{1}, B C D \beta_{2}, B C D p q$ & 4 & 8 & 6 & 0 & 12 \\
\hline & 4,$2 ; 1,0 ; 2,0$ & $A B C D, B C \beta_{1}, B C \beta_{2}$ & 4 & 8 & 0 & 0 & 12 \\
\hline & 4,$3 ; 0,2 ; 1,0$ & $C D \beta_{1}, C D p q, A B D p r$ & 4 & 8 & 5 & 0 & 12 \\
\hline
\end{tabular}

NOTE: The $2^{\left(n_{1}+n_{2}\right)-\left(k_{1}+k_{2}\right) \pm\left(b_{1}+b_{2}\right)}$ designs are labeled as "Design $=n_{1}, n_{2} ; k_{1}, k_{2} ; b_{1}, b_{2}$ " and are ordered by the number of treatment and blocking factors, $n=n_{1}+n_{2}+b_{1}+b_{2}$.

A-G and p-v are used to denote the WP and SP factors, respectively. The $i^{\text {th }}$ WP blocking variable is denoted by $\beta_{i}$. Finally, the last letter in each generator represents the added factor.

(a) The number of clear $\mathrm{C}$ main effects.

(b) The number of clear CN two-factor interactions.

(c) The number of clear CC two-factor interactions.

(e) The number of clear CN two-factor interactions tested against WP error.

(g) One number optimality criterion: Total number of clear $\mathrm{C}$ main effects and CN two-factor interactions.

* Indicates a design that is optimal with respect to the one-number criterion in the event that the design immediately preceding it in the table is not. 
Table E.4 (Cont'd)

\begin{tabular}{|c|c|c|c|c|c|c|c|}
\hline$n$ & Design & Design Generators & (a) & (b) & (c) & (e) & $(\mathrm{g})$ \\
\hline \multirow[t]{5}{*}{8} & * & $C D \beta_{1}, A p q, B C D p r$ & & & & & 13 \\
\hline & 4,$3 ; 1,1 ; 1,0$ & $A B C D, B C \beta_{1}, B C p q r$ & 4 & 12 & 0 & 0 & 16 \\
\hline & 4,$3 ; 2,0 ; 1,0$ & $A B C, A B D, A B \beta_{1}$ & 0 & 6 & 0 & 0 & 6 \\
\hline & 5,$2 ; 1,1 ; 1,0$ & $B C D E, C D \beta_{1}, A C D p q$ & 5 & 10 & 4 & 0 & 15 \\
\hline & 6,$1 ; 2,0 ; 1,0$ & $B C D E, A C D F, C D \beta_{1}$ & 6 & 6 & 0 & 0 & 12 \\
\hline \multirow[t]{14}{*}{9} & 2,$6 ; 0,3 ; 1,0$ & $A B \beta_{1}, A B q r s, A B p r t, A B p q u$ & 2 & 12 & 0 & 0 & 14 \\
\hline & 3,$4 ; 0,2 ; 2,0$ & $A B C \beta_{1}, A B C \beta_{2}, A B C p r, A B C q s$ & 3 & 12 & 3 & 0 & 15 \\
\hline & 3,$5 ; 0,3 ; 1,0$ & $A B C \beta_{1}, A B C p r, A B C q s, A B C p q t$ & 3 & 15 & 3 & 0 & 18 \\
\hline & 3,$5 ; 1,2 ; 1,0$ & $A B C, A B \beta_{1}, q r s$, prt & 0 & 15 & 0 & 0 & 15 \\
\hline & 4,$3 ; 0,2 ; 2,0$ & $C D \beta_{1}, C D \beta_{2}, C D p q, A B D p r$ & 4 & 8 & 5 & 0 & 12 \\
\hline & * & $C D \beta_{1}, C D \beta_{2}, A p q, B C D p r$ & & & & & 13 \\
\hline & 4,$3 ; 1,1 ; 2,0$ & $A B C D, B C \beta_{1}, B C \beta_{2}, B C p q r$ & 4 & 12 & 0 & 0 & 16 \\
\hline & 4,$4 ; 0,3 ; 1,0$ & $C D \beta_{1}, C D p q, A B D p r, A B C p s$ & 4 & 8 & 5 & 0 & 12 \\
\hline & * & $C D \beta_{1}, A p q, B C D p r, A B C D p s$ & & & & & 15 \\
\hline & 4,$4 ; 1,2 ; 1,0$ & $A B C D, B C \beta_{1}, B C p r, B C p q s$ & 4 & 8 & 0 & 0 & 12 \\
\hline & * & $A B C D, B C \beta_{1}, A p r, A q s$ & & & & & 15 \\
\hline & 4,$4 ; 2,1 ; 1,0$ & $A B C, A B D, A B \beta_{1}, A B q r s$ & 0 & 8 & 0 & 0 & 8 \\
\hline & 5,$2 ; 1,1 ; 2,0$ & $B C D E, C D \beta_{1}, C D \beta_{2}, A C D p q$ & 5 & 10 & 4 & 0 & 15 \\
\hline & 5,$3 ; 1,2 ; 1,0$ & $B C D E, C D \beta_{1}, C D p q, A B D p r$ & 5 & 7 & 4 & 0 & 12 \\
\hline
\end{tabular}


Table E.4 (Cont'd)

\begin{tabular}{|c|c|c|c|c|c|c|c|}
\hline$n$ & Design & Design Generators & (a) & (b) & (c) & (e) & $(\mathrm{g}$ \\
\hline \multirow[t]{8}{*}{$\overline{9}$} & * & $B C D E, C D \beta_{1}, B p q, A D p r$ & & & & & 13 \\
\hline & 5,$1 ; 2,0 ; 2,0$ & $A B C D, A B C E, B C \beta_{1}, B C \beta_{2}$ & 3 & 3 & 0 & 0 & 6 \\
\hline & 5,$2 ; 2,0 ; 2,0$ & $A B C D, A B C E, B C \beta_{1}, B C \beta_{2}$ & 3 & 6 & 0 & 0 & 9 \\
\hline & * & $B C D, A C E, B C \beta_{1}, B C \beta_{2}$ & & & & & 10 \\
\hline & 5,$3 ; 2,1 ; 1,0$ & $A B C D, A B C E, B C \beta_{1}, A B C p r$ & 3 & 9 & 0 & 0 & 12 \\
\hline & * & $B C D, A C E, B C \beta_{1}, A B p q r$ & & & & & 15 \\
\hline & 6,$1 ; 2,0 ; 2,0$ & $B C D E, A C D F, C D \beta_{1}, C D \beta_{2}$ & 6 & 6 & 0 & 0 & 12 \\
\hline & 6,$2 ; 2,1 ; 1,0$ & $B C D E, A C D F, C D \beta_{1}, A B D p$ & 6 & 12 & 0 & 0 & 18 \\
\hline
\end{tabular}


E.5 Catalog of Optimal 16-Run BFFSP RPDs via Separation: Control Factors as SP Factors 
Table E.5: Optimal 16-Run BFFSP RPDs via Separation: Control Factors as SP Factors

\begin{tabular}{|c|c|c|c|c|c|c|c|c|c|}
\hline$n$ & Design & Design Generators & (a) & (b) & (c) & (d) & $\overline{(e)}$ & $\overline{(f)}$ & $(\mathrm{g})$ \\
\hline \multirow[t]{3}{*}{6} & 1,$4 ; 0,1 ; 0,1$ & Apqrs, $A q r \delta_{1}$ & 4 & 4 & 5 & 0 & 0 & 1 & 8 \\
\hline & 2,$3 ; 0,1 ; 0,1$ & $A B p q r, A B p \delta_{1}$ & 3 & 6 & 2 & 1 & 2 & 0 & 9 \\
\hline & 3,$2 ; 1,0 ; 0,1$ & $A B C, A p q \delta_{1}$ & 2 & 6 & 1 & 0 & 0 & 1 & 8 \\
\hline \multirow[t]{7}{*}{7} & 1,$4 ; 0,1 ; 0,2$ & Apqrs, Aqr $\delta_{1}, A q r \delta_{2}$ & 4 & 4 & 5 & 0 & 0 & 1 & 8 \\
\hline & 1,$5 ; 0,2 ; 0,1$ & $A q r s$, Aprt, $A p q \delta_{1}$ & 5 & 0 & 0 & 0 & 0 & 0 & 5 \\
\hline & * & $A p s, A p q r t, A p \delta_{1}$ & & & & & & & 6 \\
\hline & 2,$4 ; 0,2 ; 0,1$ & $A B p r, A B q s, A p q \delta_{1}$ & 4 & 0 & 0 & 0 & 0 & 0 & 4 \\
\hline & * & $A B p q r, A B p q s, A B p \delta_{1}$ & & & & & & & 6 \\
\hline & 3,$3 ; 1,1 ; 0,1$ & $A B C, A p q r, A p \delta_{1}$ & 3 & 6 & 0 & 1 & 2 & 0 & 9 \\
\hline & 4,$2 ; 2,0 ; 0,1$ & $A B C, A B D, A p q \delta_{1}$ & 2 & 4 & 1 & 0 & 0 & 1 & 6 \\
\hline 8 & 1,$5 ; 0,2 ; 0,2$ & $\begin{array}{l}\text { Aqrs, Aprt, Apq } \delta_{1} \\
A p q \delta_{2}\end{array}$ & 5 & 0 & 0 & 0 & 0 & 0 & 5 \\
\hline
\end{tabular}

NOTE: The $2^{\left(n_{1}+n_{2}\right)-\left(k_{1}+k_{2}\right) \pm\left(b_{1}+b_{2}\right)}$ designs are labeled as "Design $=n_{1}, n_{2} ; k_{1}, k_{2} ; b_{1}, b_{2}$ " and are ordered by the number of treatment and blocking factors, $n=n_{1}+n_{2}+b_{1}+b_{2}$.

A-G and p-v are used to denote the WP and SP factors, respectively. The $j^{\text {th }}$ separator is denoted by $\delta_{j}$. Finally, the last letter in each generator represents the added factor.

(a) The number of clear $\mathrm{C}$ main effects.

(b) The number of clear $\mathrm{CN}$ two-factor interactions.

(c) The number of clear CC two-factor interactions.

(d) The number of clear $\mathrm{C}$ main effects tested against WP error.

(e) The number of clear $\mathrm{CN}$ two-factor interactions tested against WP error.

(f) The number of clear CC two-factor interactions tested against WP error.

(g) One number optimality criterion: Total number of clear $\mathrm{C}$ main effects and $\mathrm{CN}$ two-factor interactions.

* Indicates a design that is optimal with respect to the one-number criterion in the event that the design immediately preceding it in the table is not. 
Table E.5 (Cont'd)

\begin{tabular}{|c|c|c|c|c|c|c|c|c|c|}
\hline$n$ & Design & Design Generators & (a) & (b) & (c) & (d) & (e) & (f) & $\overline{(\mathrm{g})}$ \\
\hline \multirow[t]{7}{*}{8} & * & $\begin{array}{l}A p s, A p q r t, A p \delta_{1} \\
A p \delta_{2}\end{array}$ & & & & & & & 6 \\
\hline & 1,$6 ; 0,3 ; 0,1$ & $\begin{array}{l}\text { Aqrs, Aprt, Apqu } \\
{\text { Apqr } \delta_{1}}\end{array}$ & 6 & 0 & 0 & 0 & 0 & 0 & 6 \\
\hline & 2,$5 ; 0,3 ; 0,1$ & $\begin{array}{l}A B p r, A B q s, \text { Apqt } \\
B p q \delta_{1}\end{array}$ & 5 & 0 & 0 & 1 & 0 & 0 & 5 \\
\hline & * & $\begin{array}{l}A B p q r, A B p q s, A B p q t \\
A B p \delta_{1}\end{array}$ & & & & & & & 6 \\
\hline & 3,$4 ; 1,2 ; 0,1$ & $\begin{array}{l}A B C, A p q r, A p q s \\
A p q \delta_{1}\end{array}$ & 2 & 4 & 0 & 0 & 0 & 0 & 6 \\
\hline & 4,$3 ; 2,1 ; 0,1$ & $\begin{array}{l}A B C, A B D, A B p q r \\
A B p \delta_{1}\end{array}$ & 3 & 6 & 0 & 1 & 2 & 0 & 9 \\
\hline & 5,$2 ; 3,0 ; 0,1$ & $\begin{array}{l}A B C, A B D, A B E \\
A p q \delta_{1}\end{array}$ & 2 & 4 & 1 & 0 & 0 & 1 & 6 \\
\hline \multirow[t]{6}{*}{9} & 1,$6 ; 0,3 ; 0,2$ & $\begin{array}{l}\text { Aqrs, Aprt, Apqu } \\
{\text { Apqr } \delta_{1}, A p q r \delta_{2}}\end{array}$ & 6 & 0 & 0 & 0 & 0 & 0 & 6 \\
\hline & 1,$7 ; 0,4 ; 0,1$ & $\begin{array}{l}\text { Aqrs, Aprt, Apqu } \\
\text { pqrv, Ap } \delta_{1}\end{array}$ & 7 & 0 & 0 & 1 & 0 & 0 & 7 \\
\hline & 2,$6 ; 0,4 ; 0,1$ & $\begin{array}{l}A B p r, A B q s, A p q t \\
B p q u, A p \delta_{1}\end{array}$ & 6 & 0 & 0 & 2 & 0 & 0 & 6 \\
\hline & 3,$5 ; 1,3 ; 0,1$ & $\begin{array}{l}A B C, A p q r, A p q s \\
A p q t, A p q \delta_{1}\end{array}$ & 2 & 4 & 0 & 0 & 0 & 0 & 6 \\
\hline & 4,$4 ; 2,2 ; 0,1$ & $\begin{array}{l}A B C, A B D, A B p q r \\
A B p q s, A p q \delta_{1}\end{array}$ & 2 & 4 & 0 & 0 & 0 & 0 & 6 \\
\hline & 5,$3 ; 3,1 ; 0,1$ & $\begin{array}{l}A B C, A B D, A B E \\
A B p q r, A B p \delta_{1}\end{array}$ & 3 & 6 & 0 & 1 & 2 & 0 & 9 \\
\hline
\end{tabular}


Table E.5 (Cont'd)

\begin{tabular}{|c|c|c|c|c|c|c|c|c|c|}
\hline$n$ & Design & Design Generators & (a) & (b) & (c) & (d) & (e) & (f) & (g) \\
\hline 9 & 6,$2 ; 4,0 ; 0,1$ & $\begin{array}{l}A B C, A B D, A B E \\
A B F, A p q \delta_{1}\end{array}$ & 2 & 4 & 1 & 0 & 0 & 1 & 6 \\
\hline
\end{tabular}


E.6 Catalog of Optimal 32-Run BFFSP RPDs via Separation: Control Factors as SP Factors 
Table E.6: Optimal 32-Run BFFSP RPDs via Separation: Control Factors as SP Factors

\begin{tabular}{|c|c|c|c|c|c|c|c|c|c|}
\hline$n$ & Design & Design Generators & (a) & (b) & (c) & (d) & (e) & $(f)$ & $(\mathrm{g})$ \\
\hline \multirow[t]{5}{*}{7} & 1,$5 ; 0,1 ; 0,1$ & Aqrst, $A p r s \delta_{1}$ & 5 & 5 & 10 & 0 & 0 & 0 & 10 \\
\hline & 2,$4 ; 0,1 ; 0,1$ & $A B q r s, A p r \delta_{1}$ & 4 & 8 & 6 & 0 & 0 & 1 & 12 \\
\hline & 3,$3 ; 0,1 ; 0,1$ & $A B C p r, A p q \delta_{1}$ & 3 & 9 & 3 & 0 & 0 & 3 & 12 \\
\hline & 3,$3 ; 1,0 ; 0,1$ & $A B C, A p q r \delta_{1}$ & 3 & 9 & 3 & 0 & 0 & 0 & 12 \\
\hline & 4,$2 ; 1,0 ; 0,1$ & $B C D, A p q \delta_{1}$ & 2 & 8 & 1 & 0 & 0 & 1 & 10 \\
\hline \multirow[t]{7}{*}{8} & 1,$5 ; 0,1 ; 0,2$ & Aqrst, $A p r s \delta_{1}, A p r s \delta_{2}$ & 5 & 5 & 10 & 0 & 0 & 0 & 10 \\
\hline & 1,$6 ; 0,2 ; 0,1$ & Aqrst, Aprsu, Apq $\delta_{1}$ & 6 & 6 & 9 & 0 & 0 & 0 & 12 \\
\hline & 2,$4 ; 0,1 ; 0,2$ & $A B q r s, A p r \delta_{1}, A p r \delta_{2}$ & 4 & 8 & 6 & 0 & 0 & 1 & 12 \\
\hline & 2,$5 ; 0,2 ; 0,1$ & $A B q r s, A B p r t, A p q \delta_{1}$ & 5 & 10 & 4 & 0 & 0 & 0 & 15 \\
\hline & 3,$4 ; 0,2 ; 0,1$ & $A B C p r, A B C q s, A p q \delta_{1}$ & 4 & 12 & 0 & 0 & 0 & 0 & 16 \\
\hline & 3,$3 ; 1,0 ; 0,2$ & $A B C, A p q r \delta_{1}, A p q r \delta_{2}$ & 3 & 9 & 3 & 0 & 0 & 0 & 12 \\
\hline & 3,$4 ; 1,1 ; 0,1$ & $A B C, A p q r s, B q r \delta_{1}$ & 4 & 12 & 6 & 0 & 0 & 2 & 16 \\
\hline
\end{tabular}

NOTE: The $2^{\left(n_{1}+n_{2}\right)-\left(k_{1}+k_{2}\right) \pm\left(b_{1}+b_{2}\right)}$ designs are labeled as "Design $=n_{1}, n_{2} ; k_{1}, k_{2} ; b_{1}, b_{2}$ " and are ordered by the number of treatment and blocking factors, $n=n_{1}+n_{2}+b_{1}+b_{2}$.

A-G and p-v are used to denote the WP and SP factors, respectively. The $j^{\text {th }}$ separator is denoted by $\delta_{j}$. Finally, the last letter in each generator represents the added factor.

(a) The number of clear $\mathrm{C}$ main effects.

(b) The number of clear $\mathrm{CN}$ two-factor interactions.

(c) The number of clear CC two-factor interactions.

(d) The number of clear $\mathrm{C}$ main effects tested against WP error.

(e) The number of clear $\mathrm{CN}$ two-factor interactions tested against WP error.

(f) The number of clear CC two-factor interactions tested against WP error.

(g) One number optimality criterion: Total number of clear $\mathrm{C}$ main effects and $\mathrm{CN}$ two-factor interactions.

* Indicates a design that is optimal with respect to the one-number criterion in the event that the design immediately preceding it in the table is not. 
Table E.6 (Cont'd)

\begin{tabular}{|c|c|c|c|c|c|c|c|c|c|}
\hline$n$ & Design & Design Generators & (a) & (b) & (c) & $(\mathrm{d})$ & $(\mathrm{e})$ & (f) & (g) \\
\hline \multirow[t]{2}{*}{8} & 4,$3 ; 1,1 ; 0,1$ & $B C D, A C p q r, A B p \delta_{1}$ & 3 & 12 & 3 & 1 & 4 & 1 & 15 \\
\hline & 5,$2 ; 2,0 ; 0,1$ & $B C D, A C E, A p q \delta_{1}$ & 2 & 10 & 1 & 0 & 0 & 1 & 12 \\
\hline \multirow[t]{9}{*}{9} & 1,$6 ; 0,2 ; 0,2$ & $\begin{array}{l}\text { Aqrst, Aprsu, Apq } \delta_{1} \\
{\text { Apq } \delta_{2}}\end{array}$ & 6 & 6 & 9 & 0 & 0 & 0 & 12 \\
\hline & 1,$7 ; 0,3 ; 0,1$ & $\begin{array}{l}\text { Aqrst, Aprsu, Apqsv } \\
A_{p q r \delta_{1}}\end{array}$ & 7 & 7 & 6 & 0 & 0 & 0 & 14 \\
\hline & 2,$5 ; 0,2 ; 0,2$ & $\begin{array}{l}A B q r s, A B p r t, A p q \delta_{1} \\
A p q \delta_{2}\end{array}$ & 5 & 10 & 4 & 0 & 0 & 0 & 15 \\
\hline & 2,$6 ; 0,3 ; 0,1$ & $\begin{array}{l}\text { ABqrs, ABprt, ABpqu } \\
A p q r \delta_{1}\end{array}$ & 6 & 12 & 0 & 0 & 0 & 0 & 18 \\
\hline & 3,$5 ; 0,3 ; 0,1$ & $\begin{array}{l}A B C p r, A B C q s, A p q t \\
B p q \delta_{1}\end{array}$ & 5 & 10 & 0 & 1 & 2 & 0 & 15 \\
\hline & 3,$4 ; 1,1 ; 0,2$ & $\begin{array}{l}A B C, \text { Apqrs, Bqr } \delta_{1} \\
B q r \delta_{2}\end{array}$ & 4 & 12 & 6 & 0 & 0 & 2 & 16 \\
\hline & 3,$5 ; 1,2 ; 0,1$ & $\begin{array}{l}A B C, \text { Aqrs, Aprt } \\
\text { Apq } \delta_{1}\end{array}$ & 5 & 10 & 0 & 0 & 0 & 0 & 15 \\
\hline & 4,$4 ; 1,2 ; 0,1$ & $\begin{array}{l}B C D, A C p r, B p q s \\
B q \delta_{1}\end{array}$ & 4 & 9 & 2 & 1 & 3 & 1 & 13 \\
\hline & 5,$3 ; 2,1 ; 0,1$ & $\begin{array}{l}B C D, A C E, A B p q r \\
A B C p \delta_{1}\end{array}$ & 3 & 15 & 3 & 1 & 5 & 1 & 18 \\
\hline
\end{tabular}




\section{E.7 Catalog of Optimal 16-Run BFFSP RPDs via} Separation: Control Factors as WP Factors 
Table E.7: Optimal 16-Run BFFSP RPDs via Separation: Control Factors as WP Factors

\begin{tabular}{|c|c|c|c|c|c|c|c|}
\hline$n$ & Design & Design Generators & (a) & (b) & (c) & (e) & (g) \\
\hline \multirow[t]{3}{*}{6} & 1,$4 ; 0,1 ; 0,1$ & Apqrs, Aqr $\delta_{1}$ & 1 & 4 & 0 & 0 & 5 \\
\hline & 2,$3 ; 0,1 ; 0,1$ & $A B p q r, A B p \delta_{1}$ & 2 & 6 & 1 & 2 & 8 \\
\hline & 3,$2 ; 1,0 ; 0,1$ & $A B C, A p q \delta_{1}$ & 0 & 6 & 0 & 0 & 6 \\
\hline \multirow[t]{5}{*}{7} & 1,$4 ; 0,1 ; 0,2$ & Apqrs, $A q r \delta_{1}, A q r \delta_{2}$ & 1 & 4 & 0 & 0 & 5 \\
\hline & 1,$5 ; 0,2 ; 0,1$ & $q r s, p r t, A p q \delta_{1}$ & 1 & 5 & 0 & 0 & 6 \\
\hline & 2,$4 ; 0,2 ; 0,1$ & $A B p q r, A B p q s, A p q \delta_{1}$ & 2 & 4 & 1 & 0 & 6 \\
\hline & 3,$3 ; 1,1 ; 0,1$ & $A B C, p q r, p q \delta_{1}$ & 0 & 9 & 0 & 3 & 9 \\
\hline & 4,$2 ; 2,0 ; 0,1$ & $A B C, A B D, A p q \delta_{1}$ & 0 & 4 & 0 & 0 & 4 \\
\hline \multirow[t]{4}{*}{8} & 1,$5 ; 0,2 ; 0,2$ & $q r s, p r t, A p q \delta_{1}, A p q \delta_{2}$ & 1 & 5 & 0 & 0 & 6 \\
\hline & 1,$6 ; 0,3 ; 0,1$ & $q r s, p r t, p q u, A p q r \delta_{1}$ & 1 & 6 & 0 & 0 & 7 \\
\hline & 2,$5 ; 0,3 ; 0,1$ & $A B p q r, A B p q s, A B p q t, A p q \delta_{1}$ & 2 & 4 & 1 & 0 & 6 \\
\hline & 3,$4 ; 1,2 ; 0,1$ & $A B C, p q r, p q s, A p q \delta_{1}$ & 0 & 6 & 0 & 0 & 6 \\
\hline
\end{tabular}

NOTE: The $2^{\left(n_{1}+n_{2}\right)-\left(k_{1}+k_{2}\right) \pm\left(b_{1}+b_{2}\right)}$ designs are labeled as "Design $=n_{1}, n_{2} ; k_{1}, k_{2} ; b_{1}, b_{2}$ " and are ordered by the number of treatment and blocking factors, $n=n_{1}+n_{2}+b_{1}+b_{2}$.

$\mathrm{A}-\mathrm{G}$ and $\mathrm{p}-\mathrm{v}$ are used to denote the WP and SP factors, respectively. The $j^{\text {th }}$ separator is denoted by $\delta_{j}$. Finally, the last letter in each generator represents the added factor.

(a) The number of clear $\mathrm{C}$ main effects.

(b) The number of clear $\mathrm{CN}$ two-factor interactions.

(c) The number of clear CC two-factor interactions.

(e) The number of clear $\mathrm{CN}$ two-factor interactions tested against WP error.

(g) One number optimality criterion: Total number of clear $\mathrm{C}$ main effects and $\mathrm{CN}$ two-factor interactions.

* Indicates a design that is optimal with respect to the one-number criterion in the event that the design immediately preceding it in the table is not. 
Table E.7 (Cont'd)

\begin{tabular}{|c|c|c|c|c|c|c|c|}
\hline$n$ & Design & Design Generators & (a) & (b) & (c) & (e) & (g) \\
\hline \multirow[t]{2}{*}{8} & 4,$3 ; 2,1 ; 0,1$ & $A B C, A B D, A B p q r, A B p \delta_{1}$ & 0 & 6 & 0 & 2 & 6 \\
\hline & 5,$2 ; 3,0 ; 0,1$ & $A B C, A B D, A B E, A p q \delta_{1}$ & 0 & 4 & 0 & 0 & 4 \\
\hline \multirow[t]{8}{*}{9} & 1,$6 ; 0,3 ; 0,2$ & $\begin{array}{l}q r s, p r t, p q u, A p q r \delta_{1} \\
\operatorname{Apqr}_{2}\end{array}$ & 1 & 6 & 0 & 0 & 7 \\
\hline & 1,$7 ; 0,4 ; 0,1$ & $\begin{array}{l}q r s, p r t, p q u, p q r v \\
q r \delta_{1}\end{array}$ & 1 & 7 & 0 & 1 & 8 \\
\hline & 2,$6 ; 0,4 ; 0,1$ & $\begin{array}{l}A B p q r, A B p q s, A B p q t, A B p q u \\
A p q \delta_{1}\end{array}$ & 2 & 4 & 1 & 0 & 6 \\
\hline & * & $\begin{array}{l}A p r, A q s, A p q t, p q u \\
A p \delta_{1}\end{array}$ & & & & & 7 \\
\hline & 3,$5 ; 1,3 ; 0,1$ & $\begin{array}{l}A B C, p q r, p q s, p q t \\
A p q \delta_{1}\end{array}$ & 0 & 6 & 0 & 0 & 6 \\
\hline & 4,$4 ; 2,2 ; 0,1$ & $\begin{array}{l}A B C, A B D, A B p r, A B p q s \\
A p \delta_{1}\end{array}$ & 0 & 4 & 0 & 0 & 4 \\
\hline & 5,$3 ; 3,1 ; 0,1$ & $\begin{array}{l}A B C, A B D, A B E, A B p q r \\
A B p \delta_{1}\end{array}$ & 0 & 6 & 0 & 2 & 6 \\
\hline & 6,$2 ; 4,0 ; 0,1$ & $\begin{array}{l}A B C, A B D, A B E, A B F \\
A p q \delta_{1}\end{array}$ & 0 & 4 & 0 & 0 & 4 \\
\hline
\end{tabular}


E.8 Catalog of Optimal 32-Run BFFSP RPDs via Separation: Control Factors as WP Factors 
Table E.8: Optimal 32-Run BFFSP RPDs via Separation: Control Factors as WP Factors

\begin{tabular}{|c|c|c|c|c|c|c|c|}
\hline$n$ & Design & Design Generators & (a) & (b) & (c) & (e) & (g) \\
\hline \multirow[t]{5}{*}{7} & 1,$5 ; 0,1 ; 0,1$ & Aqrst, $A r s \delta_{1}$ & 1 & 5 & 0 & 0 & 6 \\
\hline & 2,$4 ; 0,1 ; 0,1$ & $A B q r s, A p r \delta_{1}$ & 2 & 8 & 1 & 0 & 10 \\
\hline & 3,$3 ; 0,1 ; 0,1$ & $A B C p r, A p q \delta_{1}$ & 3 & 9 & 3 & 0 & 12 \\
\hline & 3,$3 ; 1,0 ; 0,1$ & $A B C, A q r \delta_{1}$ & 0 & 9 & 0 & 0 & 9 \\
\hline & 4,$2 ; 1,0 ; 0,1$ & $A B C D, A p q \delta_{1}$ & 4 & 8 & 0 & 0 & 12 \\
\hline \multirow[t]{7}{*}{8} & 1,$5 ; 0,1 ; 0,2$ & $A q r s t, A r s \delta_{1}, A r s \delta_{2}$ & 1 & 5 & 0 & 0 & 6 \\
\hline & 1,$6 ; 0,2 ; 0,1$ & Aqrst, Aprsu, Ars $\delta_{1}$ & 1 & 6 & 0 & 0 & 7 \\
\hline & 2,$4 ; 0,1 ; 0,2$ & $A B q r s, A p r \delta_{1}, A p r \delta_{2}$ & 2 & 8 & 1 & 0 & 10 \\
\hline & 2,$5 ; 0,2 ; 0,1$ & $A B q r s, A B p r t, A p q \delta_{1}$ & 2 & 10 & 1 & 0 & 12 \\
\hline & 3,$4 ; 0,2 ; 0,1$ & $A B C p r, A B C q s, A p q \delta_{1}$ & 3 & 12 & 3 & 0 & 15 \\
\hline & 3,$3 ; 1,0 ; 0,2$ & $A B C, A q r \delta_{1}, A q r \delta_{2}$ & 0 & 9 & 0 & 0 & 9 \\
\hline & 3,$4 ; 1,1 ; 0,1$ & $A B C, A p q r s, A q r \delta_{1}$ & 0 & 12 & 0 & 0 & 12 \\
\hline
\end{tabular}

NOTE: The $2^{\left(n_{1}+n_{2}\right)-\left(k_{1}+k_{2}\right) \pm\left(b_{1}+b_{2}\right)}$ designs are labeled as "Design $=n_{1}, n_{2} ; k_{1}, k_{2} ; b_{1}, b_{2}$ " and are ordered by the number of treatment and blocking factors, $n=n_{1}+n_{2}+b_{1}+b_{2}$.

A-G and p-v are used to denote the WP and SP factors, respectively. The $j^{t h}$ separator is denoted by $\delta_{j}$. Finally, the last letter in each generator represents the added factor.

(a) The number of clear $\mathrm{C}$ main effects.

(b) The number of clear $\mathrm{CN}$ two-factor interactions.

(c) The number of clear CC two-factor interactions.

(e) The number of clear CN two-factor interactions tested against WP error.

(g) One number optimality criterion: Total number of clear $\mathrm{C}$ main effects and $\mathrm{CN}$ two-factor interactions.

* Indicates a design that is optimal with respect to the one-number criterion in the event that the design immediately preceding it in the table is not. 
Table E.8 (Cont'd)

\begin{tabular}{|c|c|c|c|c|c|c|c|}
\hline$n$ & Design & Design Generators & (a) & (b) & (c) & (e) & $(\mathrm{g})$ \\
\hline \multirow[t]{3}{*}{8} & 4,$3 ; 1,1 ; 0,1$ & $A B C D, B C p q r, B C p \delta_{1}$ & 4 & 12 & 0 & 4 & 16 \\
\hline & 5,$2 ; 2,0 ; 0,1$ & $A B C D, A B C E, A p q \delta_{1}$ & 3 & 6 & 0 & 0 & 9 \\
\hline & * & $B C D, A C E, A B p \delta_{1}$ & & & & & 10 \\
\hline \multirow[t]{11}{*}{9} & 1,$6 ; 0,2 ; 0,2$ & Aqrst, Aprsu, Ars $\delta_{1}, A r s \delta_{2}$ & 1 & 6 & 0 & 0 & 7 \\
\hline & 1,$7 ; 0,3 ; 0,1$ & Aqrst, Aprsu, Apqsv, Ars $\delta_{1}$ & 1 & 7 & 0 & 0 & 8 \\
\hline & 2,$5 ; 0,2 ; 0,2$ & $A B q r s, A B p r t, A p q \delta_{1}, A p q \delta_{2}$ & 2 & 10 & 1 & 0 & 12 \\
\hline & 2,$6 ; 0,3 ; 0,1$ & $A B q r s, A B p r t, A B p q u, A p q r \delta_{1}$ & 2 & 12 & 1 & 0 & 14 \\
\hline & 3,$5 ; 0,3 ; 0,1$ & $A B C p r, A B C q s, A B C p q t, A p q \delta_{1}$ & 3 & 15 & 3 & 3 & 18 \\
\hline & 3,$4 ; 1,1 ; 0,2$ & $A B C, A p q r s, A q r \delta_{1}, A q r \delta_{2}$ & 0 & 12 & 0 & 0 & 12 \\
\hline & 3,$5 ; 1,2 ; 0,1$ & $A B C, q r s, p r t, A p q \delta_{1}$ & 0 & 15 & 0 & 0 & 15 \\
\hline & 4,$4 ; 1,2 ; 0,1$ & $A B C D, B C p r, B C p q s, A p \delta_{1}$ & 4 & 8 & 0 & 0 & 12 \\
\hline & * & $A B C D, A p r, A q s, A p \delta_{1}$ & & & & & 15 \\
\hline & 5,$3 ; 2,1 ; 0,1$ & $A B C D, A B C E, A B C p r, A p q \delta_{1}$ & 3 & 9 & 0 & 0 & 12 \\
\hline & * & $B C D, A C E, A B p q r, A B p \delta_{1}$ & & & & & 15 \\
\hline
\end{tabular}




\section{E.9 Catalog of Optimal 16-Run BFFSP RPDs via} Mixed Blocking: Control Factors as SP Factors 
Table E.9: Optimal 16-Run BFFSP RPDs via Mixed Blocking: Control Factors as SP Factors

\begin{tabular}{|c|c|c|c|c|c|c|c|c|c|}
\hline$n$ & Design & Design Generators & (a) & (b) & (c) & $(\mathrm{d})$ & (e) & $(\mathrm{f})$ & $\overline{(\mathrm{g})}$ \\
\hline \multirow[t]{3}{*}{7} & 2,$3 ; 0,1 ; 1,1$ & $A B \beta_{1}, A B p q r, A p \delta_{1}$ & 3 & 4 & 3 & 1 & 0 & 1 & 7 \\
\hline & $*$ & $A B \beta_{1}, A B p q r, A B p \delta_{1}$ & & & & & & & 8 \\
\hline & 3,$2 ; 1,0 ; 1,1$ & $A B C, A B \beta_{1}, A p q \delta_{1}$ & 2 & 6 & 1 & 0 & 0 & 1 & 8 \\
\hline \multirow[t]{3}{*}{8} & 2,$4 ; 0,2 ; 1,1$ & $\begin{array}{l}A B \beta_{1}, A B p r, A B q s \\
A p q \delta_{1}\end{array}$ & 4 & 0 & 0 & 0 & 0 & 0 & 4 \\
\hline & $*$ & $\begin{array}{l}A B \beta_{1}, A B p q r, A B p q s \\
A p q \delta_{1}\end{array}$ & & & & & & & 6 \\
\hline & 3,$3 ; 1,1 ; 1,1$ & $\begin{array}{l}A B C, A B \beta_{1}, A p q r \\
A p \delta_{1}\end{array}$ & 3 & 5 & 0 & 1 & 1 & 0 & 8 \\
\hline \multirow[t]{3}{*}{9} & 2,$5 ; 0,3 ; 1,1$ & $\begin{array}{l}A B \beta_{1}, A B p r, A B q s \\
A p q t, A B p q \delta_{1}\end{array}$ & 5 & 0 & 0 & 1 & 0 & 0 & 5 \\
\hline & * & $\begin{array}{l}A B \beta_{1}, A B p q r, A B p q s \\
A B p q t, A p q \delta_{1}\end{array}$ & & & & & & & 6 \\
\hline & 3,$4 ; 1,2 ; 1,1$ & $\begin{array}{l}A B C, A B \beta_{1}, A p q r \\
A p q s, A p q \delta_{1}\end{array}$ & 2 & 4 & 0 & 0 & 0 & 0 & 6 \\
\hline
\end{tabular}

NOTE: The $2^{\left(n_{1}+n_{2}\right)-\left(k_{1}+k_{2}\right) \pm\left(b_{1}+b_{2}\right)}$ designs are labeled as "Design $=n_{1}, n_{2} ; k_{1}, k_{2} ; b_{1}, b_{2}$ " and are ordered by the number of treatment and blocking factors, $n=n_{1}+n_{2}+b_{1}+b_{2}$.

A-G and $\mathrm{p}-\mathrm{v}$ are used to denote the WP and SP factors, respectively. The $i^{\text {th }}$ and $j^{\text {th }} \mathrm{WP}$ blocking variable and separator are denoted by $\beta_{i}$ and $\delta_{j}$,respectively. Finally, the last letter in each generator represents the added factor.

(a) The number of clear $\mathrm{C}$ main effects.

(b) The number of clear CN two-factor interactions.

(c) The number of clear CC two-factor interactions.

(d) The number of clear $\mathrm{C}$ main effects tested against WP error.

(e) The number of clear CN two-factor interactions tested against WP error.

(f) The number of clear CC two-factor interactions tested against WP error.

(g) One number optimality criterion: Total number of clear $\mathrm{C}$ main effects and $\mathrm{CN}$ two-factor interactions.

* Indicates a design that is optimal with respect to the one-number criterion in the event that the design immediately preceding it in the table is not. 
Table E.9 (Cont'd)

\begin{tabular}{|c|c|c|c|c|c|c|c|c|c|}
\hline$n$ & Design & Design Generators & (a) & (b) & (c) & (d) & (e) & (f) & (g) \\
\hline 10 & 2,$6 ; 0,4 ; 1,1$ & $\begin{array}{l}A B \beta_{1}, A B p r, A B q s \\
A p q t, B p q u, A p \delta_{1}\end{array}$ & 6 & 0 & 0 & 2 & 0 & 0 & 6 \\
\hline & 3,$5 ; 1,3 ; 1,1$ & $\begin{array}{l}A B C, A B \beta_{1}, A p q r \\
\text { Apqs Apqt, Apq } \delta_{1}\end{array}$ & 2 & 4 & 0 & 0 & 0 & 0 & 6 \\
\hline
\end{tabular}


E.10 Catalog of Optimal 32-Run BFFSP RPDs via Mixed Blocking: Control Factors as SP Factors 
Table E.10: Optimal 32-Run BFFSP RPDs via Mixed Blocking: Control Factors as SP Factors

\begin{tabular}{|c|c|c|c|c|c|c|c|c|c|}
\hline$n$ & Design & Design Generators & (a) & (b) & (c) & (d) & (e) & (f) & $\overline{(\mathrm{g})}$ \\
\hline \multirow[t]{2}{*}{8} & 2,$4 ; 0,1 ; 1,1$ & $A B \beta_{1}, A B q r s, A p r \delta_{1}$ & 4 & 8 & 6 & 0 & 0 & 1 & 12 \\
\hline & 3,$3 ; 0,1 ; 1,1$ & $B C \beta_{1}, A B C p r, B p q \delta_{1}$ & 3 & 9 & 3 & 0 & 0 & 3 & 12 \\
\hline & 3,$3 ; 1,0 ; 1,1$ & $A B C, A B \beta_{1}, A p q r \delta_{1}$ & 3 & 9 & 3 & 0 & 0 & 0 & 12 \\
\hline & 4,$2 ; 1,0 ; 1,1$ & $B C D, B C \beta_{1}, A p q \delta_{1}$ & 2 & 8 & 1 & 0 & 0 & 1 & 10 \\
\hline \multirow[t]{5}{*}{9} & 2,$5 ; 0,2 ; 1,1$ & $\begin{array}{l}A B \beta_{1}, A B q r s, A B p r t \\
A p q \delta_{1}\end{array}$ & 5 & 10 & 4 & 0 & 0 & 0 & 15 \\
\hline & 3,$4 ; 0,2 ; 1,1$ & $\begin{array}{l}B C \beta_{1}, A B C p r, A B C q s \\
A p q \delta_{1}\end{array}$ & 4 & 12 & 0 & 0 & 0 & 0 & 16 \\
\hline & 3,$4 ; 1,1 ; 1,1$ & $\begin{array}{l}A B C, A B \beta_{1}, A B p q r s \\
A q r \delta_{1}\end{array}$ & 4 & 12 & 6 & 0 & 0 & 2 & 16 \\
\hline & 4,$3 ; 1,1 ; 1,1$ & $\begin{array}{l}B C D, B C \beta_{1}, A B C p q r \\
A C p \delta_{1}\end{array}$ & 3 & 12 & 3 & 1 & 4 & 1 & 15 \\
\hline & 5,$2 ; 2,0 ; 1,1$ & $\begin{array}{l}B C D, A C E, B C \beta_{1} \\
A p q \delta_{1}\end{array}$ & 2 & 10 & 1 & 0 & 0 & 1 & 12 \\
\hline
\end{tabular}

NOTE: The $2^{\left(n_{1}+n_{2}\right)-\left(k_{1}+k_{2}\right) \pm\left(b_{1}+b_{2}\right)}$ designs are labeled as "Design $=n_{1}, n_{2} ; k_{1}, k_{2} ; b_{1}, b_{2}$ " and are ordered by the number of treatment and blocking factors, $n=n_{1}+n_{2}+b_{1}+b_{2}$.

$\mathrm{A}-\mathrm{G}$ and $\mathrm{p}-\mathrm{v}$ are used to denote the WP and SP factors, respectively. The $i^{\text {th }}$ and $j^{\text {th }} \mathrm{WP}$ blocking variable and separator are denoted by $\beta_{i}$ and $\delta_{j}$,respectively. Finally, the last letter in each generator represents the added factor.

(a) The number of clear $\mathrm{C}$ main effects.

(b) The number of clear CN two-factor interactions.

(c) The number of clear $\mathrm{CC}$ two-factor interactions.

(d) The number of clear $\mathrm{C}$ main effects tested against WP error.

(e) The number of clear CN two-factor interactions tested against WP error.

(f) The number of clear CC two-factor interactions tested against WP error.

(g) One number optimality criterion: Total number of clear $\mathrm{C}$ main effects and $\mathrm{CN}$ two-factor interactions.

* Indicates a design that is optimal with respect to the one-number criterion in the event that the design immediately preceding it in the table is not. 
Table E.10 (Cont'd)

\begin{tabular}{|c|c|c|c|c|c|c|c|c|c|}
\hline & Design & Design Generators & (a) & (b) & (c) & (d) & (e) & (f) & 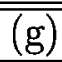 \\
\hline \multirow{5}{*}{10} & 2,$6 ; 0,3 ; 1,1$ & $\begin{array}{l}A B \beta_{1}, A B q r s, A B p r t \\
A B p q u, A p q r \delta_{1}\end{array}$ & 6 & 12 & 0 & 0 & 0 & 0 & 18 \\
\hline & 3,$5 ; 0,3 ; 1,1$ & $\begin{array}{l}B C \beta_{1}, A B C p r, A B C q s \\
A p q t, B p q \delta_{1}\end{array}$ & 5 & 10 & 0 & 1 & 2 & 0 & 15 \\
\hline & 3,$5 ; 1,2 ; 1,1$ & $\begin{array}{l}A B C, A B \beta_{1}, A q r s \\
\text { Aprt, } A p q \delta_{1}\end{array}$ & 5 & 10 & 0 & 0 & 0 & 0 & 15 \\
\hline & 4,$4 ; 1,2 ; 1,1$ & $\begin{array}{l}B C D, B C \beta_{1}, A C p r \\
B p q s, A C q \delta_{1}\end{array}$ & 4 & 9 & 2 & 1 & 3 & 1 & 13 \\
\hline & 5,$3 ; 2,1 ; 1,1$ & $\begin{array}{l}B C D, A B C E, B C \beta_{1} \\
A C p q r, A C p \delta_{1}\end{array}$ & 3 & 15 & 2 & 1 & 5 & 0 & 18 \\
\hline
\end{tabular}




\section{E.11 Catalog of Optimal 16-Run BFFSP RPDs} via Mixed Blocking: Control Factors as WP Factors 
Table E.11: Optimal 16-Run BFFSP RPDs via Mixed Blocking: Control Factors as WP Factors

\begin{tabular}{|c|c|c|c|c|c|c|c|}
\hline$n$ & Design & Design Generators & (a) & (b) & (c) & (e) & (g) \\
\hline \multirow[t]{2}{*}{7} & 2,$3 ; 0,1 ; 1,1$ & $A B \beta_{1}, A B p q r, A B p \delta_{1}$ & 2 & 6 & 0 & 2 & 8 \\
\hline & 3,$2 ; 1,0 ; 1,1$ & $A B C, A B \beta_{1}, A p q \delta_{1}$ & 0 & 6 & 0 & 0 & 6 \\
\hline \multirow[t]{2}{*}{8} & 2,$4 ; 0,2 ; 1,1$ & $A B \beta_{1}, A B p r, A B p q s, A p \delta_{1}$ & 2 & 4 & 0 & 0 & 6 \\
\hline & 3,$3 ; 1,1 ; 1,1$ & $A B C, A B \beta_{1}, p q r, A B p \delta_{1}$ & 0 & 8 & 0 & 2 & 8 \\
\hline \multirow[t]{2}{*}{9} & 2,$5 ; 0,3 ; 1,1$ & $\begin{array}{l}A B \beta_{1}, A B p r, A B p s, A B p q t \\
A p \delta_{1}\end{array}$ & 2 & 4 & 0 & 0 & 6 \\
\hline & 3,$4 ; 1,2 ; 1,1$ & $\begin{array}{l}A B C, A B \beta_{1}, p q r, p q s \\
A p q \delta_{1}\end{array}$ & 0 & 6 & 0 & 0 & 6 \\
\hline \multirow[t]{2}{*}{10} & 2,$6 ; 0,4 ; 1,1$ & $\begin{array}{l}A B \beta_{1}, A B p r, A B p s, A B p t \\
A B p q, A p \delta_{1}\end{array}$ & 2 & 4 & 0 & 0 & 6 \\
\hline & 3,$5 ; 1,3 ; 1,1$ & $\begin{array}{l}A B C, A B \beta_{1}, p q r, p q s \\
p q t, A p q \delta_{1}\end{array}$ & 0 & 6 & 0 & 0 & 6 \\
\hline
\end{tabular}

NOTE: The $2^{\left(n_{1}+n_{2}\right)-\left(k_{1}+k_{2}\right) \pm\left(b_{1}+b_{2}\right)}$ designs are labeled as "Design $=n_{1}, n_{2} ; k_{1}, k_{2} ; b_{1}, b_{2}$ " and are ordered by the number of treatment and blocking factors, $n=n_{1}+n_{2}+b_{1}+b_{2}$.

A-G and $\mathrm{p}-\mathrm{v}$ are used to denote the WP and SP factors, respectively. The $i^{\text {th }}$ and $j^{\text {th }}$ WP blocking variable and separator are denoted by $\beta_{i}$ and $\delta_{j}$,respectively. Finally, the last letter in each generator represents the added factor.

(a) The number of clear $\mathrm{C}$ main effects.

(b) The number of clear $\mathrm{CN}$ two-factor interactions.

(c) The number of clear $\mathrm{CC}$ two-factor interactions.

(e) The number of clear $\mathrm{CN}$ two-factor interactions tested against WP error.

(g) One number optimality criterion: Total number of clear $\mathrm{C}$ main effects and $\mathrm{CN}$ two-factor interactions.

* Indicates a design that is optimal with respect to the one-number criterion in the event that the design immediately preceding it in the table is not. 


\section{E.12 Catalog of Optimal 32-Run BFFSP RPDs} via Mixed Blocking: Control Factors as WP Factors 
Table E.12: Optimal 32-Run BFFSP RPDs via Mixed Blocking: Control Factors as WP Factors

\begin{tabular}{|c|c|c|c|c|c|c|c|}
\hline$n$ & Design & Design Generators & (a) & (b) & (c) & (e) & $(\mathrm{g})$ \\
\hline \multirow[t]{4}{*}{8} & 2,$4 ; 0,1 ; 1,1$ & $A B \beta_{1}, A B q r s, A p r \delta_{1}$ & 2 & 8 & 0 & 0 & 10 \\
\hline & 3,$3 ; 0,1 ; 1,1$ & $A B C \beta_{1}, A B C p r, A p q \delta_{1}$ & 3 & 9 & 3 & 0 & 12 \\
\hline & 3,$3 ; 1,0 ; 1,1$ & $A B C, A B \beta_{1}, A q r \delta_{1}$ & 0 & 9 & 0 & 0 & 9 \\
\hline & 4,$2 ; 1,0 ; 1,1$ & $A B C D, B C \beta_{1}, A p q \delta_{1}$ & 4 & 8 & 0 & 0 & 12 \\
\hline \multirow[t]{3}{*}{9} & 2,$5 ; 0,2 ; 1,1$ & $A B \beta_{1}, A B q r s, A B p r t, A p q \delta_{1}$ & 2 & 10 & 0 & 0 & 12 \\
\hline & 3,$4 ; 0,2 ; 1,1$ & $A B C \beta_{1}, A B C p r, A B C q s, A p q \delta_{1}$ & 3 & 12 & 3 & 0 & 15 \\
\hline & 3,$4 ; 1,1 ; 1,1$ & $A B C, A B \beta_{1}, A p q r s, A q r \delta_{1}$ & 0 & 12 & 0 & 0 & 12 \\
\hline & 4,$3 ; 1,1 ; 1,1$ & $A B C D, B C \beta_{1}, B C p q r, B C p \delta_{1}$ & 4 & 12 & 0 & 4 & 16 \\
\hline & 5,$2 ; 2,0 ; 1,1$ & $A B C D, A B C E, B C \beta_{1}, A p q \delta_{1}$ & 3 & 6 & 0 & 0 & 9 \\
\hline & * & $B C D, A C E, B C \beta_{1}, A p q \delta_{1}$ & & & & & 10 \\
\hline 10 & 2,$6 ; 0,3 ; 1,1$ & $\begin{array}{l}A B \beta_{1}, A B q r s, A B p r t, A B p q u \\
A p q r \delta_{1}\end{array}$ & 2 & 12 & 0 & 0 & 14 \\
\hline
\end{tabular}

NOTE: The $2^{\left(n_{1}+n_{2}\right)-\left(k_{1}+k_{2}\right) \pm\left(b_{1}+b_{2}\right)}$ designs are labeled as "Design $=n_{1}, n_{2} ; k_{1}, k_{2} ; b_{1}, b_{2}$ " and are ordered by the number of treatment and blocking factors, $n=n_{1}+n_{2}+b_{1}+b_{2}$.

A-G and $\mathrm{p}-\mathrm{v}$ are used to denote the WP and SP factors, respectively. The $i^{\text {th }}$ and $j^{\text {th }}$ WP blocking variable and separator are denoted by $\beta_{i}$ and $\delta_{j}$,respectively. Finally, the last letter in each generator represents the added factor.

(a) The number of clear $\mathrm{C}$ main effects.

(b) The number of clear $\mathrm{CN}$ two-factor interactions.

(c) The number of clear CC two-factor interactions.

(e) The number of clear CN two-factor interactions tested against WP error.

(g) One number optimality criterion: Total number of clear $\mathrm{C}$ main effects and $\mathrm{CN}$ two-factor interactions.

* Indicates a design that is optimal with respect to the one-number criterion in the event that the design immediately preceding it in the table is not. 
Table E.12 (Cont'd)

\begin{tabular}{|c|c|c|c|c|c|c|c|}
\hline$n$ & Design & Design Generators & (a) & (b) & (c) & (e) & (g) \\
\hline \multirow[t]{6}{*}{10} & 3,$5 ; 0,3 ; 1,1$ & $\begin{array}{l}A B C \beta_{1}, A B C p r, A B C q s, A B C p q t \\
A B C p q \delta_{1}\end{array}$ & 3 & 15 & 3 & 3 & 18 \\
\hline & 3,$5 ; 1,2 ; 1,1$ & $\begin{array}{l}A B C, A B \beta_{1}, q r s, p r t \\
A p q \delta_{1}\end{array}$ & 0 & 15 & 0 & 0 & 15 \\
\hline & 4,$4 ; 1,2 ; 1,1$ & $\begin{array}{l}A B C D, B C \beta_{1}, B C p r, B C p q s \\
A p \delta_{1}\end{array}$ & 4 & 8 & 0 & 0 & 12 \\
\hline & * & $\begin{array}{l}A B C D, B C \beta_{1}, A p r, A q s \\
A p q \delta_{1}\end{array}$ & & & & & 15 \\
\hline & 5,$3 ; 2,1 ; 1,1$ & $\begin{array}{l}A B C D, A B C E, B C \beta_{1}, A B C p r \\
A p q \delta_{1}\end{array}$ & 3 & 9 & 0 & 0 & 12 \\
\hline & * & $\begin{array}{l}B C D, A C E, A B \beta_{1}, A B p q r \\
A B p \delta_{1}\end{array}$ & & & & & 15 \\
\hline
\end{tabular}




\section{Bibliography}

[1] Addelman, S. (1962), "Orthogonal Main-Effects Plans for Assymetrical Factorial Experiments", Technometrics, 4, 21-46.

[2] Algina, J. (1997), "Generalization of Improved General Approximation Tests to Split-plot Designs with Multiple Between-subjects Factors and/or Multiple Within-subjects Factors", The British Journal of Mathematical and Statistical Psychology, 50, 243-252.

[3] Anderson, V.L. and McLean, R.A. (1974), Design of Experiments: A Realistic Approach, New York: Marcel Dekker, Inc.

[4] Bingham, D.R. (1998), "Design and Analysis of Fractional Factorial Split-Plot Experiments", unpublished Ph.D. thesis, Simon Fraser University, Department of Mathematics and Statistics.

[5] Bingham, D.R. and Sitter, R.R. (1999a), "Minimum-Aberration Two-Level Fractional Factorial Split-Plot Designs", Technometrics, 41, 62-70.

[6] Bingham, D.R. and Sitter, R.R. (1999b), "Some Theoretical Results for Fractional Factorial Designs", The Annals of Statistics, 27, 1240-1255.

[7] Bingham, D.R. and Sitter, R.R. (2001), "Design Issues in Fractional Factorial Split-Plot Experiments", Journal of Quality Technology, 33, 2-15.

[8] Bingham, D.R. and Sitter, R.R. (2003), "Fractional Factorial Split-Plot Designs for Robust Parameter Experiments", Technometrics, 45, 80-89.

[9] Bisgaard, S. (1994a), "Blocking Generators for Small $2^{k-p}$ Designs", Journal of Quality Technology, 26, 288-296. 
[10] Bisgaard, S. (1994b), "A Note on the Definition of Resolution for Blocked $2^{k-p}$ Designs", Technometrics, 36, 308-311.

[11] Bisgaard, S. (2000), "The Design and Analysis of $2^{k-p} \times 2^{q-r}$ Split-Plot Experiments", Journal of Quality Technology, 32, 39-56.

[12] Box, G.E.P. and Hunter, J.S. (1961), "The $2^{k-p}$ Fractional Factorial Designs", Technometrics, 3, 311-351 and 449-458.

[13] Box, G.E.P., Hunter, W.G. and Hunter, J.S. (1978), Statistics for Experimenters: An Introduction to Design, Data Analysis, and Model Building, New York: Wiley.

[14] Box, G.E.P. and Jones, S. (1992), "Split-Plot Designs for Robust Product Experimentation", Journal of Applied Statistics, 19, 3-26.

[15] Brewster, J.F. and McLeod, R.G. (2000), "On Raising a Subplot Factor to the Whole-plot Level in Blocked Fractional Factorial Split-plot Designs", American Statistical Association: 2000 Proceedings of the Section on Quality and Productivity, 110-113.

[16] Brownlee, K.A., Kelly, B.K. and and Loraine, P.K. (1948), "Fractional Replication Arrangements for Factorial Experiments with Factors at Two Levels", Biometrika, 35, 268-276.

[17] Chen, H. and Cheng, C. (1999), "Theory of Optimal Blocking of $2^{n-m}$ Designs", The Annals of Statistics, 27, 1948-1973.

[18] Chen, J., Sun, D.X. and Wu, C.F.J. (1993), "A Catalogue of Two-level and Three-level Fractional Factorial Designs with Small Runs", International Statistical Review, 61, 131-145.

[19] Chen, J. and Wu, C.F.J. (1991), "Some Results on $s^{n-k}$ Fractional Factorial Designs with Minimum Aberration or Optimal Moments", The Annals of Statistics, 19, 1028-1041.

[20] Cochran, W.G. and Cox, G.M. (1957), Experimental Designs, (2nd ed.). New York: Wiley. 
[21] Cox, D.R. (1958), Planning of Experiments, John Wiley \& Sons, New York.

[22] Daniel, C. (1959), "Use of Half-Normal Plots in Interpreting Factorial Two Level Experiments", Technometrics, 1, 311-342.

[23] Durbin, J.R. (2000), Modern Algebra: An Introduction, (4th ed.). New York: Wiley.

[24] Fisher, R.A. (1960), The Design of Experiments, New York: Hafner Publishing Company.

[25] Franklin, M.F. and Bailey, R.A. (1977), "Selecting Defining Contrasts and Confounded Effects in Two-Level Experiments", Applied Statistics, 26, 321-326.

[26] Fries, A. and Hunter, W.G. (1980), "Minimum Aberration $2^{k-p}$ Designs", Technometrics, 22, 601-608.

[27] Hedayat, A.S., Sloane, N.J.A. and Stufken, J. (1999), Orthogonal Arrays: Theory and Applications, New York: Springer-Verlag.

[28] Hedayat, A.S. and Wallis, W.D. (1978), "Hadamard Matrices and Their Applications", The Annals of Statistics, 6, 1184-1238.

[29] Hinkelmann, K. and Kempthorne, O. (1994), Design and Analysis of Experiments: Volume 1, Introduction to Experimental Design, New York: Wiley.

[30] Huang, P., Chen, D. and Voelkel, J.O. (1998), "Minimum-Aberration Two-Level Split-Plot Designs", Technometrics, 40, 314-326.

[31] Kempthorne, O. (1952), Design and Analysis of Experiments, New York: Wiley.

[32] Kirk, R.E. (1968), Experimental Design: Procedures for the Behavioral Sciences, Belmont: Brooks/Cole Publishing Company.

[33] Lindquist, E.F. (1953), Design and Analysis of Experiments in Psychology and Education, Boston: Houghton Mifflin Company.

[34] Loeppky, J.L. and Sitter, R.R. (2002), "Analyzing Unreplicated Blocked or SplitPlot Fractional Factorial Designs", Journal of Quality Technology, 34, 229-243. 
[35] Milliken, G.A. and Johnson, D.E. (1992), Analysis of Messy Data, Boca Raton: Chapman \& Hall/CRC.

[36] Montgomery, D.C. (2001), The Design and Analysis of Experiments, (5th ed.). John Wiley \& Sons, Inc.

[37] Myers, R.H. and Montgomery, D.C. (2002), Response Surface Methodology, (2nd ed.). John Wiley \& Sons, Inc.

[38] Plotkin, M. (1960), "Binary Codes with Specified Minimum Distance", IEEE Trans. Information Theory, 6, 445-450.

[39] Silverstein, A.B. (1985), "Multiple Regression Analysis of Split-plot Factorial Designs", Educational and Psychological Measurement, 45, 845-849.

[40] Sitter, R.R., Chen, J. and Feder, M. (1997), "Fractional Resolution and Minimum Aberration in Blocked $2^{n-k}$ Designs", Technometrics, 39, 382-390.

[41] Sun, D.X., Wu, C.F.J. and Chen, Y. (1997), "Optimal Blocking Schemes for $2^{n}$ and $2^{n-p}$ Designs", Technometrics, 39, 298-307.

[42] Taguchi, G. (1986), Introduction to Quality Engineering, Asian Productivity Organization, UNIPUB, White Plains, NY.

[43] Taguchi, G. (1987), System of Experimental Design: Engineering Methods to Optimize Quality and Minimize Cost, UNIPUB/Kraus International, White Plains, NY.

[44] Taguchi, G. and Wu, Y. (1980), Introduction to Off-Line Quality Control, Central Japan Quality Control Association, Nagoya, Japan.

[45] Wolfinger, R.D. and Tobias, R.D. (1998), "Joint Estimation of Location, Dispersion, and Random Effects in Robust Design", Technometrics, 40, 62-71.

[46] Wu, C.F.J. and Hamada M. (2000), Experiments: Planning, Analysis, and Parameter Design Optimization, New York: Wiley.

[47] Yates, F. (1935), "Complex Experiments", Journal of the Royal Statistical Society, Suppl. 2, 181-247. 